\title{
The Brain, Explained: A Comprehensive Theory of Brain Function
}

\author{
Ari Rappoport \\ The Hebrew University of Jerusalem, Israel \\ ari.rappoport@mail.huji.ac.il \\ www.cs.huji.ac.il/ arir \\ December 28, 2017 (first version) \\ May 8, 2018 (minor changes)
}

\begin{abstract}
Understanding brain function is one of the most important problems in human history. At present, there is no concrete theory for how the brain works. Here, a theory is presented that provides a detailed mechanistic biological account of the brain's capacities, including motor control, functional states, language, and thinking.

Brain function is managed by a well-defined response $(R)$ process th at is generally similar to the process underlying the immune system. The $\mathrm{R}$ process is strongly reflected in the brain's anatomy, physiology, and external in teractions. Different R process stages are supported by distinct excitatory networks located in different cortical layers, hippocampal fields, and bagal ganglia paths, by distinct coordination networks comprised of GABAergic interneurons, and by distinct molecular agents. The roles of norepinephrine, serotonin, dopamine and acetylcholine is to promote the alert, planning, goal-setting and execution $\mathrm{R}$ process modes, respectively. Opioids and oxytocin promote termination by success, failure, fight or $\mathrm{r}$ un, while g lucocorticoids and cannabinoids suppress acute responses to protect cells.

The R process has two instances occurring at different time scales. The millisecondscale Quax process implements the execution of hierarchical sequences of movements and thoughts, in which the selection of the next action is determined via interaction between top-down predictions and sensory inputs. The slower Need process controls the satisfaction of internal and external needs.

The theory differs from the existing standard accounts in many of the major topics (e.g., the basal ganglia, dopamine, language), and shows how cognition results from biological processes.
\end{abstract}

Keywords. Neuroscience, thalamus, basal ganglia, cortical layers, hippocampus, cerebellum, habenula, claustrum, amygdala, inhibition, predictions, automaticity, dopamine, serotonin, acetylcholine, opioids, oxytocin, CRH, glucocorticoids, cannabinoids, orexin, melanin-concentrating hormone, mirror neuron, place cells, grid cells, language, imagery, working memory, attention, consciousness, emotions. 


\section{Extended Abstract}

- We present a comprehensive mechanistic biological theory of how the brain works.

- The brain's role is to map sensory inputs (internal and external) to movement responses. Responses can be innate or adaptive. Adaptive responses can be acute (having a novel aspect) or learned (automated).

- Brain operation is managed by a well-defined response (R) process having several stages called $\mathbf{R}$ modes. The $\mathrm{R}$ process determines the brain's organization, in that different anatomical parts (e.g., auxiliary nuclei, cortical layers) and different molecular agents (e.g., dopamine, serotonin, GABA) are used by different R modes.

- There are two instances of the $\mathrm{R}$ process simultaneously executing at different time scales. The seconds to hours need $(\mathbf{N})$ process addresses needs and their satisfaction, while the millisecond scale quax $(\mathbf{Q})$ process supports execution.

- The R process proceeds as follows. The sensory input mode conveys bottom-up (BU) flow. If there is an innate response, it is executed. Otherwise, if there is a learned (automated) response, it is executed. Otherwise an acute response is executed. The acute response starts with the alert mode, which recruits resources and conveys widespread BU flow into adaptive areas. Alerts trigger the decision making (DM) mode, which conveys top-down (TD) flow that activates response candidates. Candidates compete, and the winners join response execution. The $\mathrm{R}$ process has strong similarities with the immune system process, where inflammation is the acute response.

- The brain has two execution modes, prediction and response. The prediction mode enables learned response sequences, representing the parts of the winning response that do not yet hold in external reality, including specific prepared actions and sensory predictions. The response mode supports the present reality, including perceived objects and executing actions. The $\mathrm{N}$ process has two DM modes, competition and planning, for urgent and non-urgent decisions respectively. $\mathrm{N}$ process termination can be via protection, interaction, or specific innate actions.

- The $R$ process determines the brain's organization. Excitatory neurons belong to several content networks, each supporting a different $\mathrm{R}$ mode. In cortex, the different networks occupy different layers. Sensory network: L4/6 (L3 in agranular areas). Alert network: L3b. DM network: L2/3. Prediction network: L5a (also L3 in agranular areas). Response network: L5b. In the hippocampus, the networks occupy different fields: dentate gyrus, CA3: alert/DM networks. CA1: prediction network. Subiculum: response network.

- The thalamus provides energy to cortex. Its core neurons, in both specific and nonspecific nuclei, are part of the sensory network. Its matrix neurons convey TD flow that is essential for the formation and sustenance of adaptive responses.

- The basal ganglia (BG) control cortical access to the thalamus and motor centers. The direct path is part of the DM network, and supports competition. Once competition is resolved, the direct path trains the indirect path to support acute and automated execution. (This account strongly disagrees with the standard accounts of the two paths.) 
- The $R$ process determines the brain's molecular physiology. Molecules called $\mathbf{R}$ agents (Rgens) control the R process, each Rgen promoting specific R modes and networks and suppressing competing ones. The major Rgens and their modes are as follows. Orexin, vasopressin, sex hormones, food agents: internal needs. Substance P, histamine: external needs, fight. Norepinephrine: alert. Dopamine (DA): competition, prediction (goal setting). Acetylcholine (ACh): competition, response execution. Serotonin (SER): planning, mistake. CRH: extended alert. $\mathrm{MCH}$ : interaction. Glucocorticoids, cannabinoids: energy, protection. DHEA: energy, continue. Opioids, oxytocin: termination by innates (oxytocin: satisfaction; beta-endorphin: consume; dynorphin: failure; nociceptin: fight; enkephalin: run). The hypothalamus controls the initiation and termination of the $\mathrm{N}$ process.

- The habenula supports a mistake $\mathrm{N}$ mode by rapidly switching from the execution $\mathrm{R}$ mode (DA, ACh) to the DM planning mode (SER).

- The amygdala is the innate-adaptive interface, allowing the triggering of innate responses by non-innate inputs (conditioning) and the utilization of innate capacities by adaptive responses during planning and execution.

- The $R$ process explains the role of GABAergic and glycinergic interneurons (IINs). IINs support coordination, which includes competition, sustained execution, and quax initiation. (Their role is not 'inhibition'.) IINs can be assigned to several coordination networks, the main ones being the competition coordination network $(\mathbf{C C N})$, the execution coordination network $(\mathbf{E C N})$, and the response suppression network (RSN). The networks utilize a join-or-stop (JOS) mechanism to select winners and synchronize activity.

The CCN includes SOM (Martinotti) IINs suppressing the apical dendrites of DM, prediction and response neurons, VIP IINs that suppress the SOMs to select the quax winners via disinhibition of these networks, and neurogliaform IINs that suppress quax losers. The ECN includes PV and CCK soma-targetting (basket) IINs that synchronize execution to sustain it. The RSN includes chandelier PV IINs that rapidly suppress responses.

- Cortex is arranged in nodes, tightly connected microcolumns supporting vertical flow between the content networks. Nodes are the fundamental units of learned (adaptive) cortical representations.

- Execution involves the activation of a set of neurons called a quax. The Q process manages execution via quax transitions in which neurons join and leave the executing quax, thereby supporting hierarchical sequences. The executing quax contains action nodes active in response mode that drive movement, and object nodes active in prediction mode (i.e., their prediction network is active but their respone network is not) that represent the action's sensory goals. The executing actions excite (mobilize) all action and object nodes that have been useful in the past. When the active goals are attained, the resulting sensory inputs cause the nodes representing goals to switch to response mode, and select the next action via competition among the mobilized actions. In this way, hierarchical sequences emerge from sensory-action $\mathrm{R}$ process interactions without being centrally managed.

- Competition and its resolution explain event-related potentials (ERPs), fMRI BOLD, and repetition suppression. DM planning explains working memory. Mobilization 
explains mirror neurons. Transitions explain EEG oscillations and the attentional blink. Goals explain place and grid cells and theta phase precession.

- Learned (automated) responses are promoted each time responses are executed, starting from the initial acute response that trains a learned response while it executes. Learned responses are supported by the response network, by predictions in the prediction network and anticipations in the DM network, by the indirect BG path, and by the high-affinity Rgen receptors and autoreceptors (mainly D2R, alpha2-NER, and SER1/4/7).

- Dopamine promotes the competition and prediction (goal-directed execution) R modes (together called decision), including immediate charging approach innate actions, via D1R, and learned transitions via D2R. (The role of DA is not 'reward'.) Serotonin promotes the planning $\mathrm{R}$ mode, by suppressing innate responses ('emotions') and executing responses via SER1R, exciting the DM network and the CCN via SER2R and SER3R, exciting memory/planning areas via SER4/6, and exciting motor circuits via SER7. (The role of SER is not 'happiness'.)

- Language and other forms of communication are managed by the standard Q process. The only thing unique about them is that they use non-neuronal modalities (audio, movement, touch) in order to bridge the neuronal gap between organisms. Syntax is the interface between non-linguistic goal nodes and gap bridging actions, and its hierarchy and sequencing capacities are implemented as in all other actions.

- Imagery and thinking occur during the planning R mode and are implemented by the standard $\mathrm{Q}$ process. Motor imagery involves the activation of gamma motoneurons, which form the innate part of the prediction network.

- The state of $\mathrm{R}$ modes is accessible to adaptive actions via interoceptive sensory inputs reaching cortical nodes (mainly the insula and mPFC). Awareness (consciousness) and emotions (feelings) result from Q processes that report events and R mode states.

- The theory explains other important phenomena that are discussed in depth elsewhere, including plasticity (learning), postnatal development, sleep, and brain disorders (autism, addiction, MS, AD, etc.). 


\section{Contents}

List of Figures

$\begin{array}{llr}1 & \text { Introduction } & 8\end{array}$

2 Overview: General Principles \& Processes 9

2.1 Function ........................... 9

2.2 General architecture . . . . . . . . . . . . . . . . . . . . 9

2.3 The $\mathrm{R}$ process . . . . . . . . . . . . . . . . . . 10

3 The Innate-Adaptive Axis 15

3.1 Ibrain, abrain, innates, valence . . . . . . . . . . . . . . 15

3.2 The amygdala: the innate-adaptive interface . . . . . . . . . . . 16

4 The Process Axis: R Mode Networks $\quad 18$

4.1 The innate sensory network . . . . . . . . . . . . . . . . 18

4.2 The innate response network . . . . . . . . . . . . . . 18

4.3 The adaptive sensory network . . . . . . . . . . . . . 20

4.4 The alert network . . . . . . . . . . . . . . . . . 22

4.5 The decision making $(\mathrm{DM})$ network $\ldots \ldots \ldots \ldots . \ldots . \ldots . \ldots 22$

4.6 The adaptive response network . . . . . . . . . . . . . . 22

4.7 The prediction (goals) network . . . . . . . . . . . . 22

4.8 The thalamic TD network . . . . . . . . . . . . . . 23

4.9 The basal ganglia $(\mathrm{BG})$ : gating execution . . . . . . . . . . . . . . 23

4.10 The hippocampus . . . . . . . . . . . . . . . . 24

4.11 Cognitive interpretation: predecision, reality, goals $\ldots \ldots \ldots 26$

5 The Content Axis: Representations 27

5.1 Representations . . . . . . . . . . . . . . . . . . 27

5.2 Nodes . . . . . . . . . . . . . . . . . . . . . 28

5.3 Object, action, valence areas . . . . . . . . . . . . . . . . . . . . . . . . . . . . . 29

5.4 Events, episodic memory . . . . . . . . . . . . . . 30

6 The Q Process: Motor Control 31

6.1 Action goals, sequences \& hierarchy . . . . . . . . . . . . . . . 32

6.2 Coordination: competition, synchrony, initiation . . . . . . . . . . 35

6.3 Coordination networks . . . . . . . . . . . . . . . 37

6.4 ERPs, repetition suppression, predictions . . . . . . . . . . . . . 39

6.5 The cerebellum . . . . . . . . . . . . . . . . . . 41

6.6 Attention: orienting, the claustrum . . . . . . . . . . . . . 42

6.7 Conditional wait . . . . . . . . . . . . . . . . . . 43

6.8 Working memory . . . . . . . . . . . . . . . . . . 44

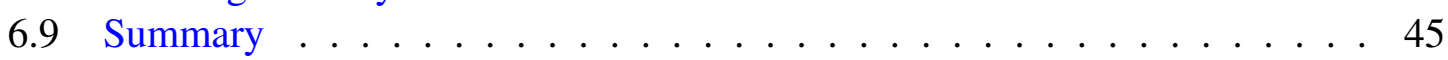

7 The N Process: Functional States 4

7.1 The hypothalamus: $\mathrm{N}$ mode hub . . . . . . . . . . . . . . . . 46

7.2 Internal need: orexin (OX), arginine vasopressin (AVP), sex hormones . . . 46

7.3 Alert: norepinephrine $(\mathrm{NE}) \ldots \ldots \ldots$. . . . . . . . . . . 48

7.4 Extended alert: corticotropin-releasing hormone $(\mathrm{CRH}) \ldots \ldots$. . . . . . 50 
7.5 Decision making, planning: serotonin (SER) . . . . . . . . . . . . 51

7.6 Competition, prediction, goal-setting: dopamine (DA) . . . . . . . . . 53

7.7 Response execution, attention: acetylcholine (ACh) . . . . . . . . . . 56

7.8 Pain, aggression, mating: substance $\mathrm{P}(\mathrm{SP})$, histamine . . . . . . . . . . 58

7.9 Mistake: the habenula . . . . . . . . . . . . . . . . . . 60

7.10 Consume: beta-endorphin (BEND) . . . . . . . . . . . . . 61

7.11 Satisfaction: oxytocin $(\mathrm{OT}) \ldots \ldots \ldots \ldots$. . . . . . . . . . . 64

7.12 Failure, withdrawal: dynorphin $(\mathrm{DYN}) \ldots \ldots \ldots$. . . . . . . 65

7.13 Aggression: nociceptin (NOC, N/OFQ) . . . . . . . . . . . 66

7.14 Running: enkephalin $(\mathrm{ENK}) \ldots \ldots \ldots 66$

7.15 Interaction: melanin-concentrating hormone $(\mathrm{MCH}) \ldots \ldots . . \ldots 67$

7.16 Energy, protection: glucocorticoids (GCs), endocannabinoids (ECBs), DHEA . . . . . . . . . . . . . . . . 68

8 Language $\quad 70$

8.1 The nature of language . . . . . . . . . . . . . . . 70

8.2 Representations \& areas . . . . . . . . . . . . . . . 70

8.3 Processing . . . . . . . . . . . . . . . . . 72

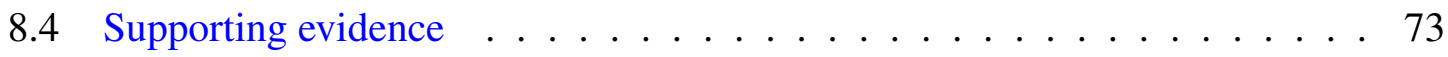

9 Internal Cognition $\quad \mathbf{7 5}$

9.1 Awareness, consciousness . . . . . . . . . . . . . . 75

9.2 Emotions . . . . . . . . . . . . . . . . . . 77

9.3 Familiarity \& recollection . . . . . . . . . . . . . . . . . 78

9.4 Imagery, thinking . . . . . . . . . . . . . . . . . . . . . . . 79

9.5 True \& false recollection . . . . . . . . . . . . . . . . . 83

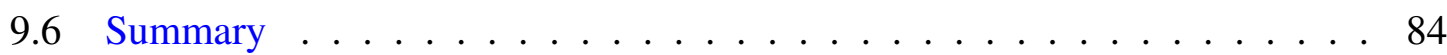

10 Additional Evidence $\quad 84$

10.1 Action observation, mirror neurons . . . . . . . . . . . . . . . 84

10.2 Place \& grid cells, theta phase precession . . . . . . . . . . . . . 85

10.3 Conditioning, generalization \& extinction . . . . . . . . . . 86

10.4 EEG oscillations . . . . . . . . . . . . . . . . . . . . 88

10.5 Attentional paradigms . . . . . . . . . . . . . . . . . 89

11 Discussion $\quad 90$

12 Final Words $\quad 94$

13 References $\quad 94$

14 Supplementary Material $\quad \mathbf{1 2 6}$

14.1 Abbreviations and new terms . . . . . . . . . . . . . 126

14.2 Method . . . . . . . . . . . . . . . . . . 128

14.3 Generative linguistics, recursion, etc. . . . . . . . . . . . . . . 129

\section{List of Figures}

1 The $\mathrm{R}$ process (general version). . . . . . . . . . . . . . . . . . . . 10

2 The $\mathrm{R}$ process (detailed brain modes). . . . . . . . . . . . . . . . . 12 
3 The amygdala. . . . . . . . . . . . . . . . . 17

4 Abrain architecture (including the basal ganglia and thalamus). . . . . . . . 19

5 Intra-node connections (micro-circuit, including coordination networks). . . 20

6 The hippocampus. . . . . . . . . . . . . . . . 25

7 The cerebellum. . . . . . . . . . . . . . . 41 


\section{Introduction}

How the brain works is one of the few truly major questions in human history. Following Newton, Einstein, Darwin and many others, we possess a good understanding of many aspects of the physical world and of life. However, we do not understand how living organisms act, think and feel. These capacities are known to be controlled by the brain, but currently there exists no brain theory or anything close to it, despite the enormous amount of research effort invested. Much progress has been made in our knowledge of cellular properties, but our understanding of higher brain levels (networks, systems, behaviors) is vague at best [Grillner, 2014], usually phrased in terms of abstract functions of large brain areas rather than through concrete mechanisms [Brown, 2014]. The brain is the most complex structure in nature; some researchers think that we may never understand how the human brain works [Adolphs, 2015], or that knowledge would only gradually accumulate, over many years [Koch and Marcus, 2014]. Large concerted data collection and measurement projects are widely viewed as essential for moving forward [Olds, 2016].

This situation is not surprising, given that a full theory of brain function faces the formidable task of explaining at least the following capacities: goal-directed movements and movement sequences; perception, including the identification of external objects and events; mental imagery (imagination, thinking, planning); attention; emotions; the nature of awareness (consciousness); motivated vs. automatic and habitual behavior; predictions and surprise; reward and aversion; innate responses (threat/fear, consummatory acts), their generalization and extinction; language; familiarity, novelty, and true and false recollection; episodic memory; learning; sleep.

In addition, a comprehensive theory must explain the function of the following components and phenomena: the different classes of excitatory and inhibitory neurons; cortical layers and columns; the major areas and structures, including the basal ganglia, thalamus, cerebellum, amygdala, hypothalamus, hippocampus, habenula, claustrum, and cortical areas such as the insula and Broca's area; neuromodulators and neuropeptides such as dopamine, serotonin, acetylcholine, norepinephrine, opioids, cannabinoids, and oxytocin; synchrony and oscillations; ERP components; place cells and mirror neurons. The theory must also provide plausible accounts for disorders.

In this text I present a theory of brain ${ }^{1}$ function accounting for all of the items listed above. It is a mechanistic biological theory, phrased in terms of concrete cellular processes. As such, the theory is refutable and provides a wealth of concrete predictions. The main idea is that brain function can be fully explained using a single response (R) process that has two brain instantiations, one driven by internal and external needs and the other managing response execution. The brain's anatomy and molecular physiology are directly reflected by the R process, which is generally similar to the process guiding the immune system.

The methodology I used to develop the theory was to gather as many relevant facts as possible, and try to arrange them into a coherent picture. To find the facts, I examined a large number of papers and books ${ }^{2}$. I did not conduct experiments; when forming a theory prediction, I searched for papers describing experiments that could refute or support it. The resulting theory turned out to be very different from existing accounts of specific phenomena in most important areas (e.g., the basal ganglia, GABA, dopamine, serotonin). I refer to the present (2017) version of the theory as R17, to distinguish it from inevitable future updates.

The present paper aims for a level of description that would be expected in textbooks.

\footnotetext{
${ }^{1}$ The theory covers the central nervous system (CNS); I use 'brain' for brevity.

${ }^{2}$ See supplementary material.
} 
Due to the scope of the subject, it is clearly impossible to go much deeper than that or to cite even a small part of the relevant literature here. The paper covers the brain aspects directly related to the main functional process, leaving topics having a different nature for other publications. These topics include brain learning and plasticity (including sleep, postnatal development, lateralization, and gender differences), and a mechanistic account of brain disorders. s.

The theory does not cover brain evolution, prenatal development, and species differences, but it uses supporting data from these fields where appropriate.

\section{Overview: General Principles \& Processes}

\subsection{Function}

The central nervous system (brain, for brevity) controls the organism's interaction with the body-external environment. In particular, it drives and manages the external movements involved in satisfying survival-related needs, both internal needs (food, minerals, water, reproduction, waste disposal) and external ones (threats from other organisms and natural forces). Other aspects of such needs are managed by other body systems (e.g., the metabolism and reproduction systems). Another major need type, body-internal threats, is managed by the immune system. The brain and these systems cooperate in fulfilling their functions.

\subsection{General architecture}

The brain is a non-linear complex system, comprised of a large number of functional cells called neurons (an order of $10^{10}$ in humans) that interact through many-to-many $\left(10^{1}-10^{5}\right)$ connections. Neurons possess branching input and output processes called dendrites and axons, respectively. Most connections (synapses) are point-to-point, with axons connecting to dendrites at precise locations (dendritic spines).

Brain function is based on electrically charged chemical agents (ions). The main positively charged agents are calcium, potassium, sodium and zinc ions, while the main negatively charged agents are chloride ions. Neurons are normally polarized (i.e., there is a voltage difference across their membrane). When a neuron is depolarized (hyperpolarized) it is said to be excited (suppressed, inhibited). Inter-neural communication is done via energy flow, the release of agents that act on membrane receptors to induce the opening of ion channels and other cell internal processes. When ion channels are open, the direction of ion movement is determined by concentration gradients across the cell's plasma membrane. When a neuron is excited above a certain threshold, a strong response (action potential (AP)) of about one millisecond occurs that induces the axonal release of agents, which in turn affects the polarity of the axon's targets. In general, depolarization by dozens of source neurons is needed to get a target neuron fire an AP.

The main excitatory agent is glutamate (glut), which mainly acts through two types of receptors, AMPA and NMDA. An important distinction is that between calcium-permeable (CP) and calcium-impermeable (CI) receptors, since $\mathrm{CP}$ receptors allow rapid excitation due to the substantially higher concentration of extracellular Ca2+. NMDARs and some AMPARs are CP. The main inhibitory agents are GABA and glycine.

The brain also contains non-neuronal cells called glia cells, which support neuronal metabolism and protection and the reuptake and recycling of agents. Since these functions are relevant to brain learning and disorders, glia are not addressed in this paper. 


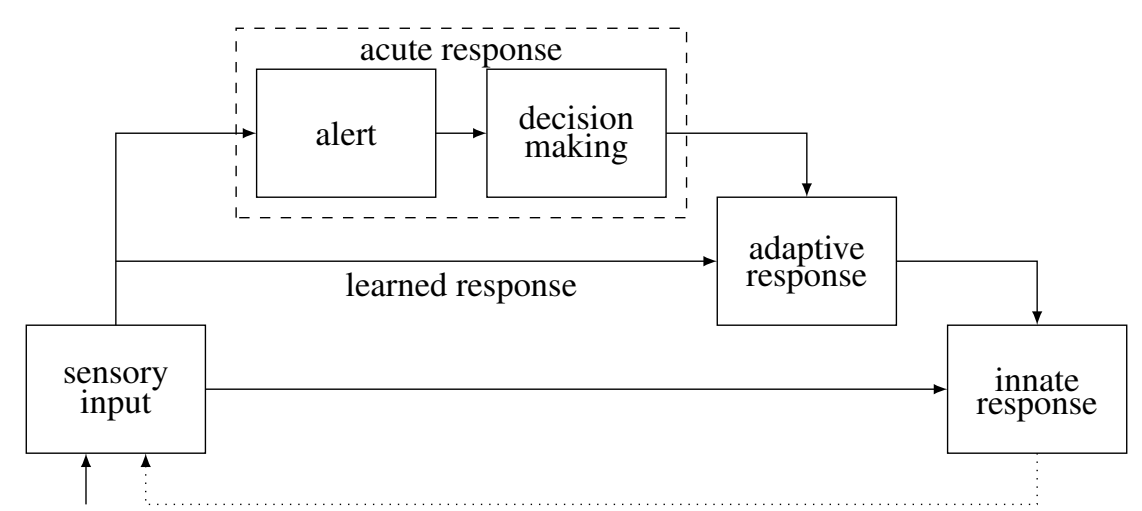

Figure 1: The general $\mathrm{R}$ process (without brain-unique aspects). Arrows indicate excitatory flow. The dotted line indicates that response execution affects incoming sensory inputs.

The brain is a learning system, continuously modifying its parts and connections to optimize its performance. We distinguish between innate brain capacities, genetically hard-coded by evolution and present in all healthy members of a species, and adaptive capacities, different between organisms and acquired during a particular organism's lifetime. Adaptive capacities that have been optimized are called automated, and capacities that have a novel aspect are called acute or flexible.

\subsection{The $\mathbf{R}$ process}

The major contribution of $\mathrm{R} 17$ is in describing brain operation as following a general response ( $\mathbf{R})$ process, comprised of a sequence of stages called $\mathbf{R}$ modes. The main $R$ modes utilize different neuronal networks, and are promoted by different molecular agents (neuromodulators). The $\mathrm{R}$ process provides a relatively simple conceptual framework through which virtually all brain phenomena can be understood.

There are five basic $\mathrm{R}$ modes (Figure 1): sensory input, innate response, alert, decision making (DM), and adaptive response. The process starts with sensory input. If there is an innate response to this input, it is executed. If there is no innate response, or if the input persists after executing it, flow reaches adaptive areas. If there is a learned response, it is executed. Otherwise, an acute mode finds and executes a flexible response. The acute mode is comprised of two stages, an alert that directs resources to the event, and a decision making (DM) response that recruits response candidates (existing learned or innate responses that respond to some feature of the input) and lets them compete against each other. A focused adaptive response (decision) emerges from the recruited alternatives and is executed, using innate responses (usually low-level ones).

The $\mathrm{R}$ process has an obvious similarity to the processes underlying other biological systems. In the immune system, there is also a distinction between innate and adaptive immune responses, DM candidates are adaptive immune cells ( $\mathrm{T}$ and $\mathrm{B}$ cells) that respond to some partial component of the pathogen (relatively short amino acid sequences displayed with major histocompatibility complex molecules by antigen presenting cells), the acute response is called inflammation, and the system learns the emerging focused response. In evolution, DM candidates are generated by variation, and the competition process is called natural selection. It is our view that the $\mathrm{R}$ process underlies all successful living systems and that an $\mathrm{R}$ process account of a system can substantially improve its understanding. This General Theory of Life is discussed elsewhere. 
Brain operation is best described as having two instances of the $\mathrm{R}$ process unfolding at different time scales (intermediate and short term), addressing needs ( $\mathrm{N}$ process) and response execution ( $\mathrm{Q}$ process) respectively. The brain implementation of the $\mathrm{R}$ process has several brain-unique modes (Figure 2). Response execution is divided into two modes, prediction and response, to support goal setting and response sequences. DM has two sub-modes, competition and planning, for urgent and non-urgent decisions. The $\mathrm{N}$ process has mistake, interaction, extended alert and energy management modes.

The different $\mathrm{R}$ modes use largely non-overlapping neuronal content networks. Innate areas have sensory and response networks. Adaptive areas have sensory, alert, decision making (DM), prediction and response networks, which in cortex are located in different layers (L4/6, L3b, L2/3, L5a, and L5b respectively). The response network is the one directly inducing muscle contraction and movement.

Each $\mathrm{R}$ mode is promoted by one or more molecular $\mathbf{R}$ agents (Rgens). Rgens modify neuronal excitability via selective neuronal receptors, usually several receptor types per agent. Due to intracellular signaling, extracellular propagation, and distribution through the cerebrospinal fluid (in some cases), Rgen effect on brain execution can be spatially and temporally extended. In general, the Rgens promoting acute responses also promote automated responses, using lower amounts and different (high affinity) receptors. We say that Rgens mold the neuronal terrain to channel flow, thereby promoting their R modes.

The $\mathbf{N}$ process. The intermediate time scale need $(\mathbf{N})$ process takes between seconds to hours, its stages ( $\mathbf{N}$ modes) reflecting the state of internal and external needs (Table 1). Internal needs are conveyed by specific agents sensed by neurons, thereby increasing the excitability of the neuronal paths involved in the satisfaction of specific needs. The alert $\mathrm{N}$ mode commonly follows surprises that trigger innate orienting of attention and innate sympathetic responses that recruit energy resources. If an alerting event is classified as unthreatening, the interaction mode terminates the alert and by default stimulates interaction with the external object. If the situation is potentially threatening but does not trigger specific innate or acute responses, the extended alert mode sustains a prolonged alert and triggers energy management.

There are two types of decision making. Planning (non-urgent DM) is promoted by SER. Competition (urgent DM), as well as execution, are promoted by DA and ACh. Both Rgens widely excite response candidates, push towards making a response, and sustain its execution. DA has a greater role in need-driven decisions (i.e., the $\mathrm{N}$ process) and prediction (goal setting), while ACh promotes attended execution (i.e., the Q process, see below). A mistake signal can be communicated at any time to stop execution and switch back to non-urgent decision making mode (see habenula below).

Termination of the acute mode can be due to interaction, protection, or execution of innates $^{3}$. Protection is induced by the energy provision mode after excessive recruitment of energy resources. Such negative feedback is needed since a prolonged acute response may damage cells via oxidative stress and other processes. Protection is done by suppressing adaptive responses and by promoting innate responses, which are energetically efficient, without promoting any specific one. Protection competes with another energy management mode, continue, which optimizes energy utilization of acute responses.

Execution of specific innates is the common way in which acute execution is terminated, where each major innate is promoted by a different opioid agent or oxytocin. There are two success modes involving the satisfaction of internal needs, both comprising a brief boosting of responses followed by prolonged relaxation. The consume success mode uses

${ }^{3}$ For brevity, we use 'innates' rather than 'innate responses'. 


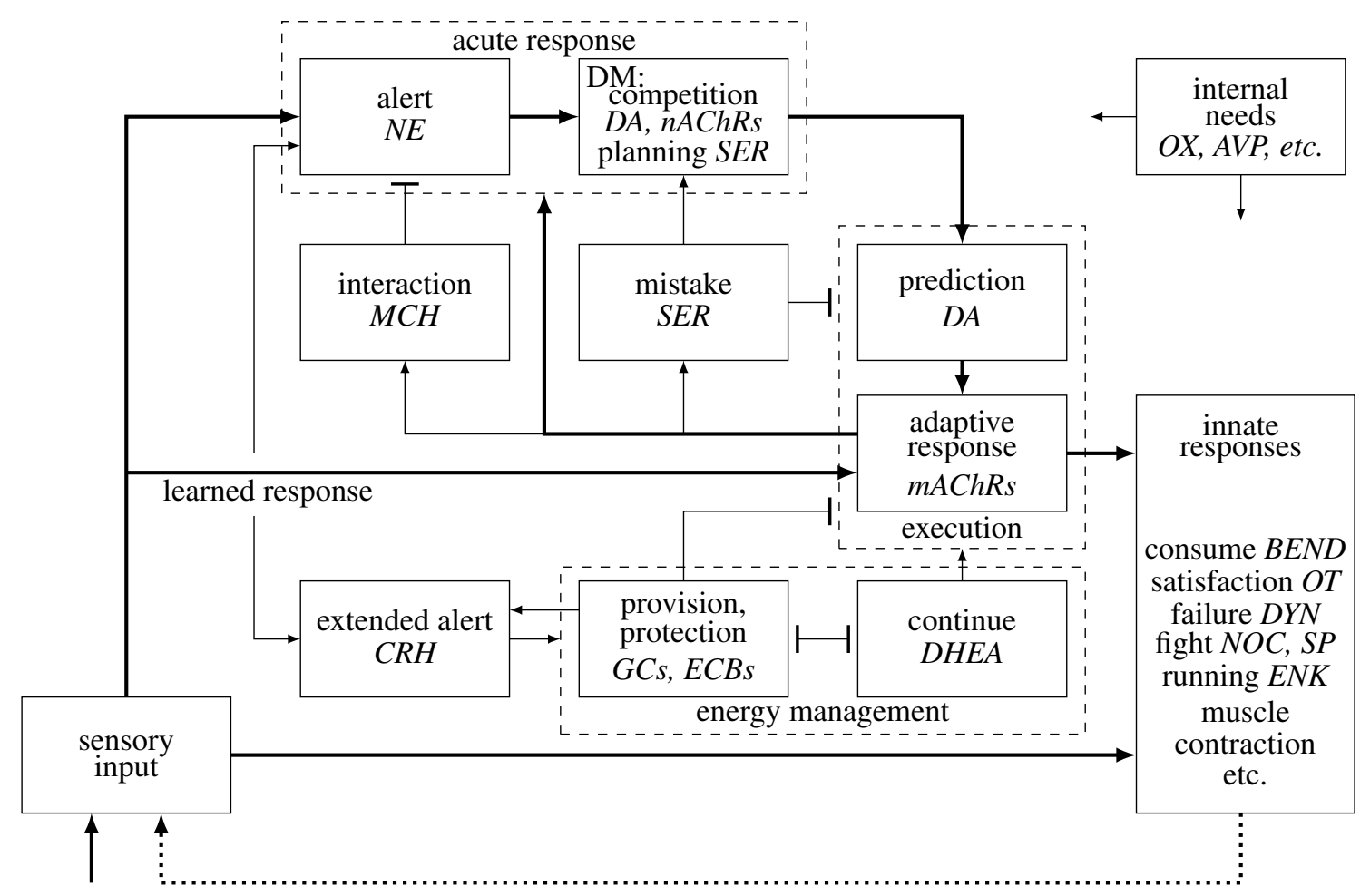

Figure 2: The brain's $\mathrm{R}$ process. Arrows indicate excitatory flow, $\mathrm{T}$ junctions indicate suppression. Mode Rgens are shown in italics. The brain-unique aspects beyond the general $\mathrm{R}$ process are: (i) internal needs mold the neuronal terrain to affect response formation (the specific excitation of $\mathrm{N}$ process DA by OX is not shown); (ii) response execution includes two modes, prediction and response, with the former unidirectionally exciting the latter; (iii) decision making (DM) includes two sub-modes, competition (urgent DM) and planning (non-urgent DM), which excite prediction; (iv) a mistake mode suppresses execution and promotes renewed DM; (v) an extended alert mode reciprocally excites alert and promotes energy management; (vi) energy management includes two competing sub-modes, provision (protection) and continue. Provision suppresses excessive execution (negative feedback), sustains the part of extended alert that sustains alerts, and uses negative FB on the part of extended alert that triggers energy management (not shown in the figure); (vii) an interaction mode suppresses alert in case the input is not threatening; (viii) innate responses include muscle contraction, success (consume, satisfaction), failure (freeze, withdraw), fight (aggression), flight (run), etc. The internal circuits of innate responses are not shown. The dotted line indicates that responses modify incoming sensory inputs.

The connections serving both the $\mathrm{N}$ process and the $\mathbf{Q}$ process are shown in thick lines. Note the thick connection from the adaptive to the acute response, which enables the inner loop forming non-urgent DM (planning).

All modes except sensory inputs are promoted by specific Rgens.

All modes are implemented by dedicated anatomical parts of the brain (with some overlap): sensory input (innate labeled lines and the thalamic core), innate responses (muscles, PAG, amygdala CeM, medulla, pons, spinal cord, hypothalamus, etc), alert (alert network), DM (DM network, direct BG path, DG+CA3), mistake (habenula), prediction (prediction network, CA1), response (response network, indirect BG path), internal needs (pituitary, adrenal cortex, gonadals, gut, etc), energy management (pituitary, adrenal cortex), extended alerts (amygdala CeL, paraventricular hypothalamus $\mathrm{CRH}$ neurons). 


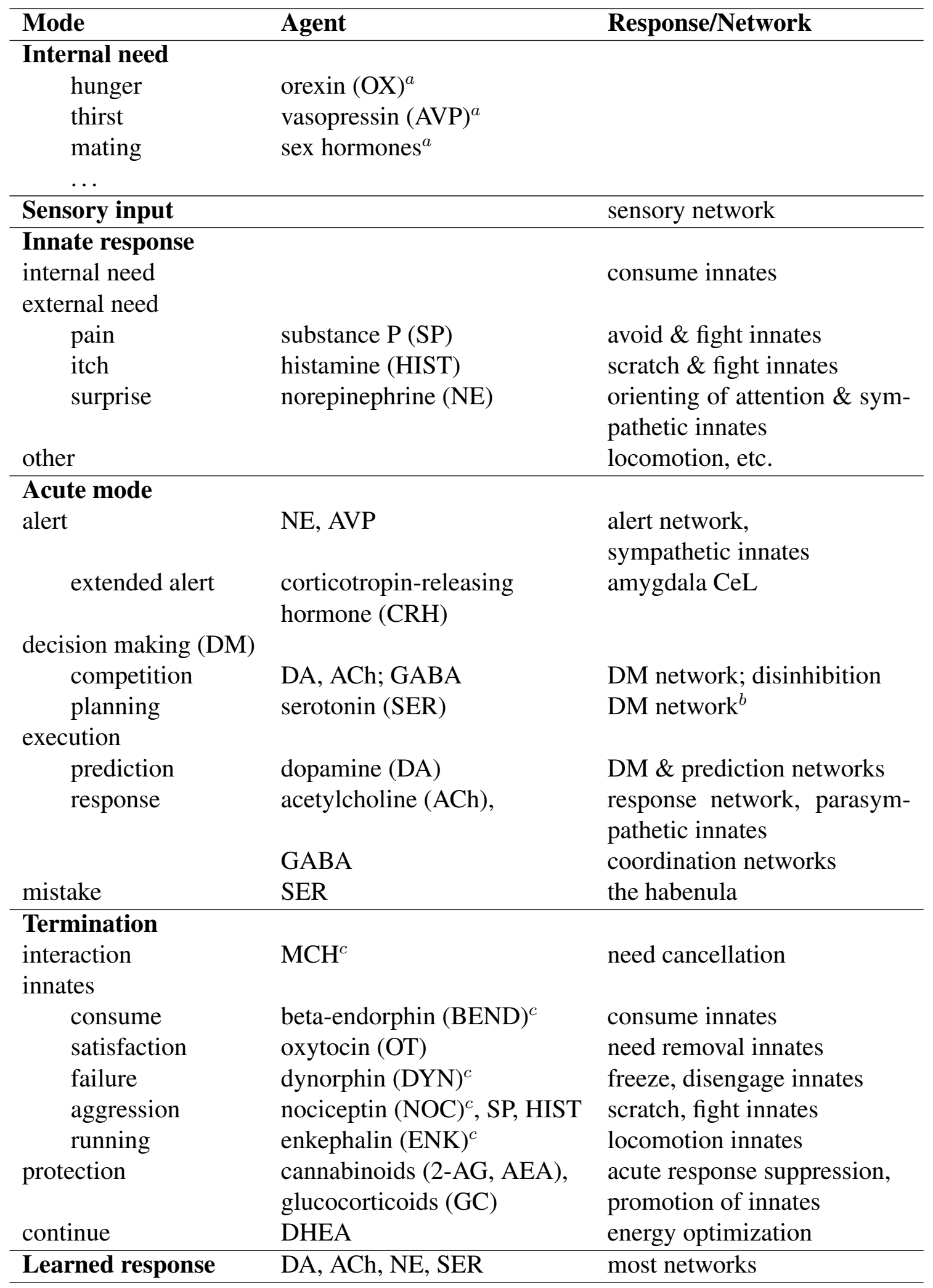

Table 1: The $\mathrm{N}$ process, including generic $\mathrm{R}$ modes and $\mathrm{N}$ modes unique to the brain. For each $\mathrm{N}$ mode, its Rgen(s), responses, and networks are indicated.

$a$ : Important food, thirst and sex Rgens are not listed, see the need $\mathrm{N}$ mode section.

$b$ : Planning also involves other networks, see the imagery section.

$c$ : BEND, DYN, ENK and NOC are opioids. MCH: melanin-concentrating hormone. 
innates that utilize skeletal muscles, while the satisfaction mode mostly uses innates that utilize smooth muscles and entail the secretion and intake of fluids. The failure mode utilizes disengage and freeze innates. The fight (aggression) mode utilizes fight innates to deal with the event, and the running mode streamlines chase and flight via locomotion innates ${ }^{4}$.

In terms of cognitive experience, the $\mathrm{N}$ modes are an important component of the states underlying the meaning of the word emotions (see internal cognition below).

The $\mathbf{Q}$ process. Post-competition $\mathrm{N}$ modes can involve a large number of lower level sensor-response (or stimulus-response) (SR) mappings. We use the term quax (plural quacia $^{5}$ ) to denote the set of neurons participating in an active post-competition SR mapping. Quax formation and execution are managed by the second $\mathrm{R}$ process instance, the millisecond scale quax $(\mathbf{Q})$ process. The space of the SR mappings made possible by a specific brain anatomy (i.e., neurons and their connectivity structure) is called the underlying quax space (UQS).

All execution (innate, learned, non-urgent DM, acute) is implemented by a Q process. The Q process supports all aspects of cognitive execution, both external (movement, language) and internal (thinking, imagination). In the external $Q$ process, action neurons activate action goals represented by sensoriobject neurons active in prediction mode. When the goals are met, the sensoriobject representations switch to response mode and select the next action among competing ones. In this way, the prediction network facilitates the execution of hierarchical action sequences. In the internal Q process (thinking) some details may be different (see internal cognition below).

The $\mathrm{Q}$ process is assisted by GABAergic interneurons arranged in coordination networks. Coordination is a term that includes competition, synchrony and initiation. There are three coordination networks: the competition coordination network $(\mathbf{C C N})$ comprises the non-PV-expressing IINs in the superficial networks, the execution coordination network (ECN) comprises basket IINs (PV and CCK), and the response suppression network comprises PV IINs that target the axon initial segment. The DM stage of the Q process uses competition to determine which of the candidates activated during DM would be included in the precise UQS subset that the executing quax uses. GABAergic neurons mediate competition via a winner-takes-all mechanism called join or stop (JOS). Competition involves disinhibition, the inhibition of a default response suppression. GABAergic neurons also support quax initiation via brief excitation, and sustained quax execution through synchrony.

Generally, the $\mathrm{N}$ process determines what needs to be done, while the Q process manages how things are done. Since these two instances of the R process run simultaneously but at different time scales, they can be at different $\mathrm{R}$ modes at any particular time. Specifically, the $\mathrm{N}$ process can be in acute execution mode while employing learned $\mathrm{Q}$ process motor actions, and conversely, the automated $\mathrm{N}$ mode can involve $\mathrm{Q}$ process alerts triggered by low level surprises. Moreover, the $\mathrm{Q}$ and $\mathrm{N}$ instantiations of the same $\mathrm{R}$ mode can have very different natures. Notably, $Q$ process DM involves pre-quax competition between neurons, while $\mathrm{N}$ process DM can involve competition between established quacia (thinking/planning, see below).

Learning. The brain continuously activates plasticity mechanisms to support behavioral

\footnotetext{
${ }^{4}$ From a predator's point of view, the $\mathrm{N}$ process is not terminated by fight and chase but by subsequent consume innates. However, if successful, fight and flight constitute termination from the prey's perspective. In addition, like the other innate termination modes, they are promoted by opioids.

${ }^{5}$ Pronounced k'wa-sha. I thank Deborah Gera for help with the Latin.
} 
adaptivity. R17 includes a detailed account of this topic, $\mathbf{R}$ learning. Brain learning has one overarching principle, to facilitate the future activation of executed quacia. Each time a response is executed, learning mechanisms are invoked to improve its subsequent execution, starting from the initial acute response that trains a learned one (e.g., see the BG paths).

Since execution is managed by the $\mathrm{R}$ process, learning tightly reflects the $\mathrm{R}$ process. In turn, learning strongly affects the $\mathrm{R}$ process, through short-term plasticity and longterm automaticity. Nonetheless, the learning process is not an instance of the $\mathrm{R}$ process as the $\mathrm{Q}$ and $\mathrm{N}$ processes are, since it generally does not involve neuronal firing that mediate responses. There is a learning stage in which neurons fire, sleep, but is it driven by distributed intracellular processes, not by sensory inputs. For this reason we describe R learning elsewhere. Learning results in reduced acute responses and a quick suppression of quax neurons, discussed under automaticity in various parts of the paper.

Since learning agents such as calcium and zinc can damage cells (via apoptotic processes, oxidative stress, etc.), learning is intimately tied to neuroprotection and metabolism. Glia cells are important participants in these processes and are described elsewhere as well.

Regarding learned content, innate responses are triggered by sensory features, but adaptive responses are commonly made according to feature combinations called objects. A major role of brain learning is to learn objects and the appropriate responses to them.

The brain's anatomy reflects these $\mathrm{R}$ process notions and has three main axes (or dimensions). The innate-adaptive axis is the main flow axis connecting sensors to responses, and includes an innate-adaptive interface. The process axis includes the different flow networks supporting the main $\mathrm{R}$ modes. The content axis supports a rich quax space via a multitude of specific representations.

\section{The Innate-Adaptive Axis}

\subsection{Ibrain, abrain, innates, valence}

The main flow path in the brain is along the axis connecting sensors to innate and then to adaptive responses. The axis connects posterior (caudal, tail) and anterior (rostral, nose) parts of the nervous system. The posterior and anterior parts implement innate and adaptive responses and are called ibrain and abrain, respectively. The sensor-innate-adaptive direction is called bottom-up (BU), and the opposite direction is called top-down (TD).

The ibrain consists of neuronal sensory and response paths extending throughout the body, spinal cord, and brainstem. It supports hard-wired actions (innates), triggered by specific sensory inputs in a hard-coded manner. Innates are species-specific and formed by evolution. Key innate structures are the periaqueductal gray (PAG), the trigeminal nuclei, the parabrachial nucleus (PBN), the nucleus of the solitary tract (NTS), the pons, the medulla, the vagus nerve and the hypothalamus $(\mathrm{HT})^{6}$.

The abrain supports adaptive capacities, acquired during the organism's lifetime. Its main components are the cortex, hippocampus, extended amygdala, thalamus, basal ganglia (BG), cerebellum, claustrum, and habenula ${ }^{7}$. Adaptive actions can directly access muscles and can utilize innate circuits.

Common innates include smell-triggered approach movements, consume actions (those involved in eating, drinking and mating), avoid actions (e.g., pain- and smell-triggered re-

\footnotetext{
${ }^{6}$ The HT is described in the N process section.

${ }^{7}$ Note that many subcortical structures support adaptive, not innate, responses (see discussion).
} 
flexes, itch triggered scratching, flight, freeze, vomit), locomotion, orienting of attention, and need initiation and resolution. The latter two belong to a large family of innates used for communication with other bodily systems (e.g., the baroreceptor and immune reflexes) and are conveyed via the autonomic nervous system (ANS). Most brain-ANS communication is done via the HT. Locomotion (running) and aggression (fight) innates are used in both consume and avoid situations. Running innates are called by different names depending on the animal's role, chase for predators and flight for prey.

We say that sensory inputs and learned objects that preferentially yield avoid responses have a negative valence, and those that yield consume and need termination responses have a positive valence. Locomotion, attention and aggression innates do not possess inherent valence, but they may carry positive or negative valence when associated with specific objects or situations.

\subsection{The amygdala: the innate-adaptive interface}

The amygdala is a set of nuclei in the deep temporal lobe. The amygdala serves as the interface between the abrain and ibrain, extending each system with the capacities of the other. It provides adaptivity to the innate system by allowing innates to be activated by non-innate sensory inputs, via a specific type of learning called conditioning (below). We use innate+ to denote the resulting extended innate system. In the other direction, the amygdala allows the utilization of innates by adaptive responses, in two ways. First, it provides the abrain with access to object-innate mappings, letting adaptive responses be guided by object valence (e.g., during decision making). Second, it allows the abrain to select or disconnect specific innates for execution, as part of an adaptive response.

The amygdala has two main regions (Figure 3), the central (CeA) and basolateral (BLA) complexes, each having two main nuclei (centrolateral $(\mathrm{CeL})$, centromedial $(\mathrm{CeM})$, lateral (LA), basolateral (BL)), and two smaller medial and cortical olfactory nuclei. CeA CeL is innervated mostly by sensory inputs (direct interoceptive and somatosensory pain inputs, thalamic inputs via LA) and BLA, and projects to CeM. CeM projects to innate motor and autonomic output areas, including the PAG, the trigeminal nerve [Lazarov et al., 2011] and the hypothalamus. BLA receives inputs from the thalamus and cortex, projects unidirectionally to $\mathrm{CeA}$ and the $\mathrm{BG}$, and is mutually connected with cortex and the hippocampus. Its projections to cortex are mostly into layers 1 and 2, and may be stronger than sensory inputs from the thalamus [Timbie and Barbas, 2014]. Thus, BLA strongly affects the results of competitions. BLA and CeA projection neurons are glutamatergic and GABAergic, respectively.

CeA supports (and stores) executed SR mappings (quacia) by receiving their triggering sensory inputs (into CeL) and allowing their responses (via CeM->PAG). The ventrolateral (vl) PAG has a population of inhibitory interneurons (IINs) that are tonically active, suppressing excitatory vIPAG neurons that project to medulla neurons innervating spinal motor neurons. These IINs are suppressed by CeM neurons to disinhibit the excitatory response paths, allowing various types of competing innate responses [Tovote et al., 2016] ${ }^{8}$. Thus, innate+ responses are supported by CeA, while BLA stores the associations between sensoriobject flow (from the thalamus into LA) and the executed innates (via BLA-CeM projections). Both CeA and BLA alert cortex when innates are executed, CeA via its HT projections (see the alert and extended alert N modes), and BLA directly. Through BLA's bidirectional cortical connections and its BG projections, it lets adaptive responses use in-

\footnotetext{
${ }^{8}$ The amygdala is commonly viewed as being essential for executing innate actions. However, innates may be possible with a lesioned amygdala [Antoniadis and McDonald, 2001].
} 


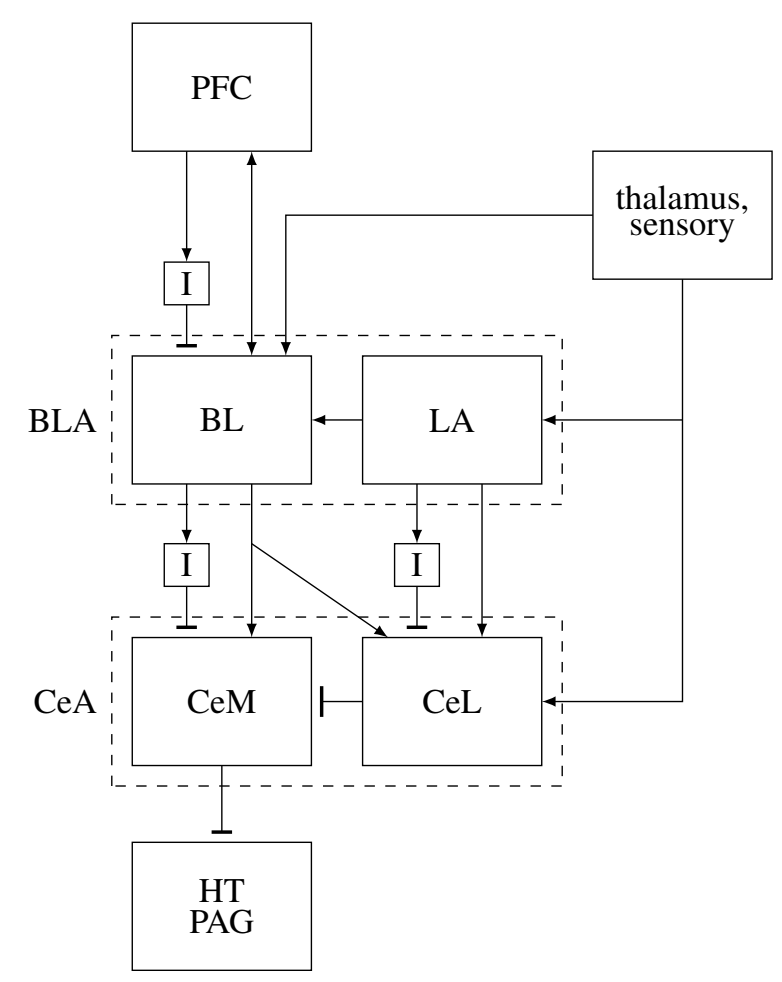

Figure 3: The amygdala. CeA controls the activation of innate responses (at the PAG, HT and other centers) via disinhibition. BLA serves as the interface between adaptive responses (mostly from PFC) and innate ones, via BLA-CeA projections. Only the main connections are shown. BNST, hippocampus, BG connections are not shown, as well as the cortical olfactory nuclei and circuits involving PKCD and non-PKCD neurons (see conditioning). ICMs are idealized. CeA: central complex. CeM: centromedial part of CeA. CeL: centrolateral part of CeA. BLA: basolateral complex. BL: basolateral part of BLA. LA: lateral part of BLA. I: intercalated masses (see BEND/consume section). HT: hypothalamus.

nates and be guided by object valence. The PAG is a BLA-CeM controlled switch that selects which innate response is activated, with specific selections corresponding to R termination modes and mediated by termination agents (opioids, OT etc.).

The amygdala is grouped with the bed nucleus of the stria terminalis (BNST) to form the extended amygdala. The BNST is closer to the abrain than the amygdala is, and focuses on place valence (see below). Acute situations strongly affect extended amygdalar plasticity, increasing spontaneous neuronal firing and enhancing sensoriobject-response connections and cortical and BG excitation. As a result, situations in which the amygdala or BNST are acutely activated, such as highly stressful events and consuming drugs of abuse, can induce a state of chronic alert and a chronic favoring of innates over adaptive responses (i.e., reduced control). This explains many of the symptoms of post-traumatic stress disorder (PTSD), anxiety, and addiction. The amygdala is further discussed below under valence areas, $\mathrm{N}$ modes, and threat (fear) conditioning and extinction. 


\section{The Process Axis: R Mode Networks}

Each location in the posterior-anterior flow axis contains separate neuronal networks supporting different $\mathrm{R}$ process modes. This is done differently in cortex, ibrain, the thalamus, the BG and the hippocampus. The cortical networks are located in different vertical layers (see Figures 4 and 5). The identification of networks associated with $\mathrm{R}$ modes is a major contribution of R17.

Flow network neurons are excitatory via glutamate ${ }^{9}$. Neurons in each cortical network are bidirectionally interconnected to support quax stability through synchronous firing, but in all networks (except perhaps the response network) flow in one direction (BU or TD) is stronger than in the other [Markov et al., 2014]. The different networks show different patterns of Rgen receptor expression. These patterns provide strong support for our description of the networks, and are discussed in the $\mathrm{N}$ mode section.

\subsection{The innate sensory network}

Sensory input originates in sensors located throughout the body. The brain has three types of sensors, for exteroception (light, sound, taste, smell, and touch), proprioception (the state of the muscles and tendons) and interoception (the state of various internal body tissues). The input flows through the ibrain and splits into two streams, ibrain and abrain. The former innervates brainstem centers where it can activate innates and continue to the amygdala CeL, while the latter continues to cortex through the so-called specific thalamic nuclei (except olfaction, which reaches cortex directly). In most cases, each sensor type has its own path ('labeled line') until it reaches cortex (e.g., taste [Chen et al., 2011]). This yields topographic maps, i.e., cortical organization (receptive fields) that preserves in some way the relative structure of the input ${ }^{10}$. Because sensory flow reaches innate circuits before reaching adaptive areas, innate responses enjoy a latency advantage.

Proprioception is unique in that it has a special non-moving mechanism for stopping its inputs. Proprioceptive inputs arise from sensory neurons in the classical spinal cord motor unit. These neurons are excited by muscle spindles, which are sensitive to muscle length and contraction velocity, and by Golgi tendon organs sensitive to tension. The spinal cord in mammals has neurons called gamma motoneurons (gMTNs), which innervate muscle spindles to set the threshold determining sensory neuron activation (in other species, this function is done by axon collaterals of the main motoneurons, aMTNs). Thus, gMTNs can adjust thresholds to prevent sensory neurons from stopping firing ${ }^{11}$ or to suppress their firing. We view gMTNs as the innate part of the prediction network (see imagery below). If there exist gMTNs that are solely excited by ibrain neurons, these gTMNs constitute an innate prediction network.

\subsection{The innate response network}

Sensory inputs innervate ibrain motor centers where they can trigger innate actions. Probably the simplest full quax is the motor unit, whose response network consists of alpha motor neurons (aMTNs) that innervate muscles to yield muscle contraction. In the stretch reflex,

\footnotetext{
${ }^{9}$ Disinhibition neurons, which are GABAergic, are naturally viewed as belonging to flow networks in some areas (the BG and cerebellum), and to coordination networks in other areas (cortex, amygdala CeM).

${ }^{10}$ This specificity of sensory input can be contrasted with inputs that the brain receives via the circulation, whose role is to affect $\mathrm{R}$ modes.

${ }^{11}$ This role is also assigned to beta MTNs.
} 


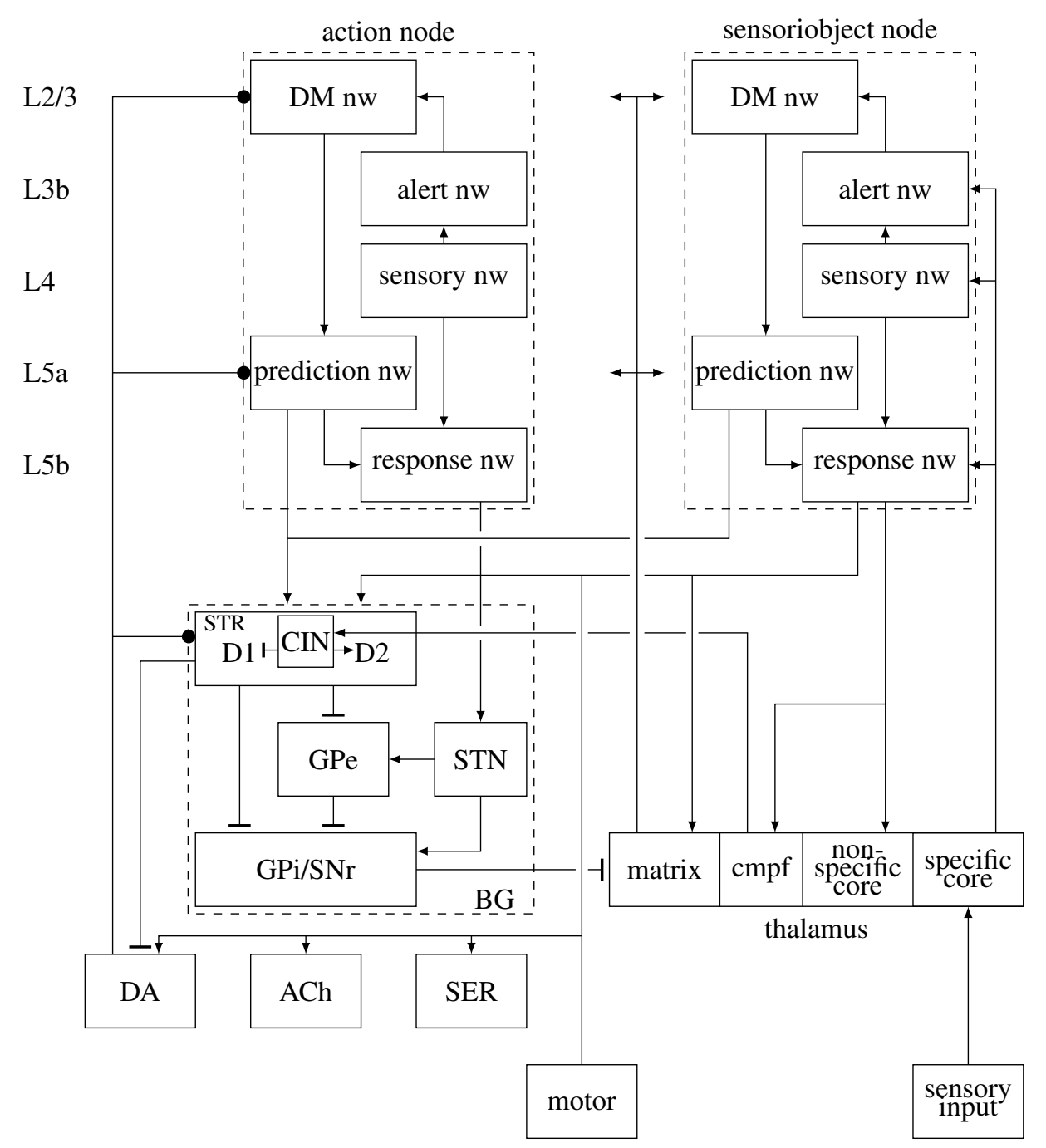

Figure 4: Abrain architecture, including the BG, thalamus, two cortical nodes (cortical columns), in primary sensory cortex (right) and frontal cortex (left), and three Rgen nuclei. The cerebellum, hippocampus and amygdala are not shown. Arrows, T junctions and circles indicate excitatory flow, GABAergic flow, and Rgen release respectively.

The BG output nuclei suppress the thalamic matrix, which projects diffusely to the internal networks to sustain non-automated execution. The prediction and response networks project to the STR (to both the D1 and D2 paths), to recruit the BG and the thalamus to allow quax formation and execution. The response network also projects to the STN, thalamus, Rgen nuclei and motor centers. The STN sustains the BG response path (GPe, $\mathrm{GPi} / \mathrm{SNr}$ ). The thalamic cmpf nuclei excite the STR CINs when sensory input arrives. The CINs generally excite the D2 path to allow responses suppress the D1 path to allow decision making.

To avoid clutter, some connections are not shown. These include the thalamic non-specific core projections to the action node, the intra-flow network connections, cortical ACh and SER projections, GPe projections to the STR and STN, DM network innervation of the alert network, BG projections to motor centers, cerebellum, and non-DA Rgen nuclei, the different projections of striosome and STR matrix neurons onto DA nuclei, and amygdala projections to the STR. The L6 part of the sensory network is not shown.

BG: basal ganglia. CIN: cholinergic interneurons. cmpf: center median-parafascicular complex of the thalamus. D1 (D2): the STR projection neurons expressing D1R (D2R). GP: globus pallidus (i: internal, e: external). nw: network. SNr: subtantia nigra reticulata. STR: striatum. STN: subthalamic nucleus. 


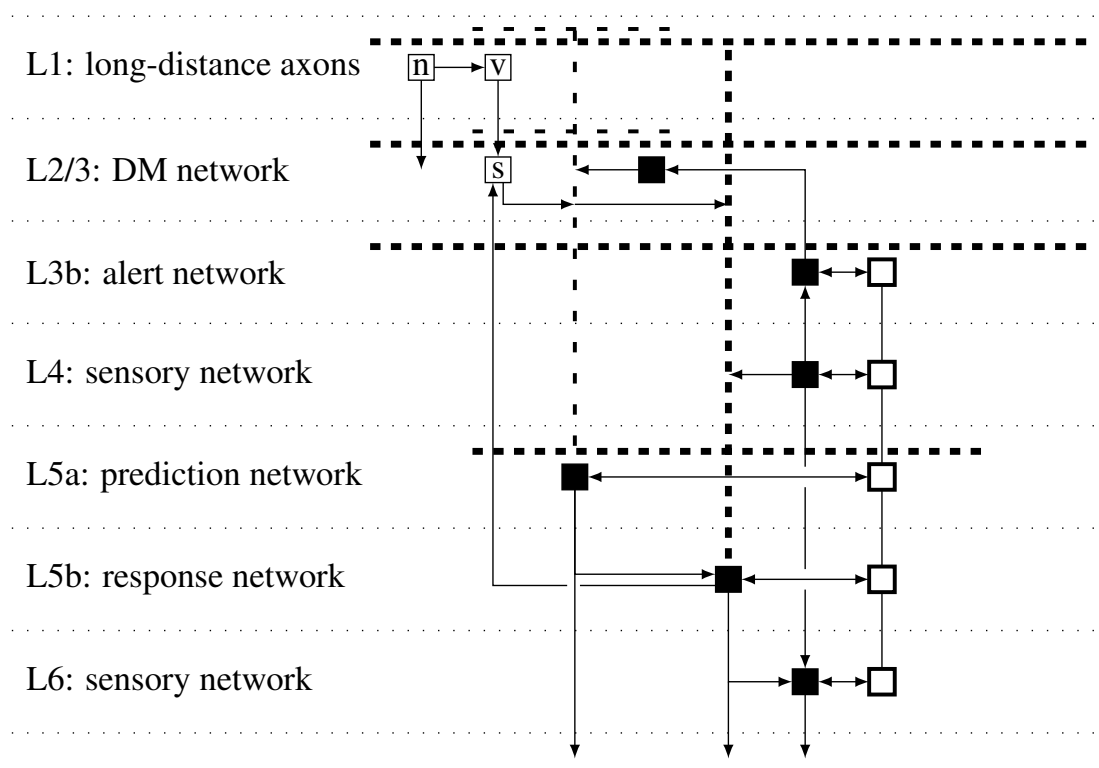

Figure 5: Intra-node connections. Only the main patterns are shown. Thalamic inputs, inter-node connections and the RSN are not shown. The horizontal spacing between neurons is exaggerated. Filled squares: excitatory neurons. The thick (slender) tufted dendritic tree of the response (prediction) neuron is shown in thick (thin) dashed lines. Empty squares: coordination IINs. The execution coordination network (ECN) is on the right in thick lines. ECN interconnections are shown without arrows to emphasize gap junction coupling. The competition coordination network $(\mathrm{CCN})$ is on the left (s: SOMs, v: VIPs, n: NGFCs). NGFCs target all cell types, depicted by a single general arrow. The deep networks have subcortical projections, to motor centers, thalamus, and BG (response network), BG (prediction network), and thalamus (L6 sensory network).

forced limb movement yields muscle stretch, which activates sensory neurons. These excite their agonistic motoneuron to yield movement in the opposite direction and stabilize posture. In normal movement, sensory neurons excite inhibitory interneurons that suppress the antagonistic muscle ${ }^{12}$. Higher level innate actions utilize more complex circuits, some of which known as 'central pattern generators' (e.g., for locomotion).

As the muscle contracts, the sensory neurons reporting its status reduce their firing rates. Normally, response neurons that directly generate movement require proprioceptive sensory inputs to be able to do so [Galán et al., 2015]. Without proprioceptive inputs, the abrain can learn to activate motor neurons adaptively via a different modality (e.g., vision), but this requires extensive training [Cole and Paillard, 1995]. As noted above, gMTNs can be used to ensure continued proprioceptive inputs to enable movement.

\subsection{The adaptive sensory network}

Most sensory input reaches the abrain via the thalamus, a large bilateral set of nuclei at the center of the brain (see Figure 4). Excitatory thalamic neurons can be classified into core neurons, which project to middle cortical layers in a spatially focused manner and express parvalbumin (PV) in primates ${ }^{13}$, and matrix neurons, which are mostly present in nonspecific nuclei, project to layers 1-3 (L1-3) and L5 in a spatially diffuse manner, and express

\footnotetext{
${ }^{12}$ This is the simplest type of competition, see below.

${ }^{13} \mathrm{PV}$ is an execution marker, see coordination networks below.
} 
calbindin $(\mathrm{CB})^{14}$ [Clascá et al., 2012]. The thalamus also contains coordination networks. The adaptive sensory network consists of the thalamocortical core ${ }^{15}$, excitatory cortical L4 neurons, and corticothalamic L6 neurons. Core neurons project mainly to sensory network neurons in $\mathrm{L} 4^{16}$, but also to alert and response network neurons [Constantinople and Bruno, 2013]. The L4 sensory neurons project vertically to excitatory neurons in L6 (as well as to the alert network), and these project back to the thalamus ${ }^{17}$, closing an input flow loop that ensures quax stability.

The thalamus contains two types of nuclei, specific and non-specific. The specific nuclei are those conveying raw sensory flow to cortex. The non-specific nuclei are innervated by axon collaterals of cortical response network neurons and by the L6 sensory network neurons, and have core and matrix parts projecting to cortex. We view the non-specific thalamic core as part of the adaptive sensory network. Cortical response neurons, which are the main input of core non-specific neurons, can be activated by surprising BU sensory input (see bursts under the alert $\mathrm{N}$ mode). When this occurs, their activation is not part of a response, but part of still unanswered input. By innervating the non-specific thalamic core, response neurons convey such BU flow to higher level cortical areas.

Agranular \& dysgranular areas. L4 conveys thalamic core flow to the alert network in L3b (see below). Given that the thalamic core also projects directly to L3b, it is not immediately clear why L4 is needed. Indeed, some cortical areas are agranular (lack L4) or dysgranular (have a smaller L4).

The main R17 observation in explaining this phenomenon is that in areas that must yield rapid responses to sensory input, L4 is redundant and only slows down the Q process. There are two types of such areas: those supporting low level motor control, and those providing immediate responses to alerts. Indeed, primary motor cortex (M1) is agranular, and premotor cortex, whose responses should be fast but not as fast as those of M1, is dysgranular. Similarly, entorhinal cortex (EC), which supports locomotion (see grid cells below), is agranular. In areas of this type, L3 is the sensory network (being the one that receives sensory input), and it also behaves like the prediction network. The role of the prediction network is to support action sequences by channelling responses to inputs to the next action (see below). When responses are very quick, predictions should arrive to the sensory network, because the additional synapse through the prediction network would slow down execution. Indeed, the hippocampus provides evidence that EC L3 behaves like a prediction network (see below).

The second type of agranular area in shown by medial prefrontal cortex (mPFC) and parts of the insula, both of which receive amygdalar alerts, as well as by the supplementary eye field, which supports attention orienting movements. Granular PFC areas support long term planning and execution and exist only in primates.

Thus, the role of L4 is to enable a longer DM process supporting longer response latencies. The separation of L4 from L3b allows L4 competition to be resolved while L3b competition (i.e., decision making) is still being held. This way, L4 allows sharp (focused) inputs to drive a coarse (disinhibited) DM process.

\footnotetext{
${ }^{14} \mathrm{CB}$ is the fastest $\mathrm{Ca} 2+$ buffering protein, according with participation in acute responses.

${ }^{15}$ Thus, the thalamic core is the thalamic sensory (or BU) network. Generally, the networks that convey BU flow are the sensory and alert networks, but the thalamus does not have an alert network.

${ }^{16}$ See also agranular/dysgranular areas below.

${ }^{17}$ L6 also has corticoclaustral and corticocortical populations. The claustrum attends to sensory input (see below), while the latter may connect the sensory network across cortex, as done in the other networks.
} 


\subsection{The alert network}

The alert network consists of pyramidal neurons in L3b (the lower part of L3). They are innervated by the sensory network, both vertically by L4 and by the thalamic core, and by the amygdala BLA. Alert neurons excite the L2/3 DM network vertically, and neighboring L4 neurons horizontally in all directions, mostly BU but also TD [Markov et al., 2014]. Thus, the network conveys unanswered BU flow that quickly extends to more frontal and ventral areas to drive decision making. We refer to the sensory and alert networks together as the external or BU networks.

\subsection{The decision making (DM) network}

The DM network consists of the pyramidal neurons in L2/3. They are vertically innervated by the alert and sensory networks, and can also be innervated by the prediction and response networks through long-distance connections [Rempel-Clower and Barbas, 2000]. They innervate other DM neurons horizontally through local and long-distance connections, mostly in the TD direction, and through projections to the other hemisphere (i.e., they are commissural, callosal being the largest subgroup).

Since the alert and the DM networks are adjacent and are activated in the same R process stage (DM), their neurons are often intermingled such that all of $\mathrm{L} 2 / 3$ seems to be a single layer. Careful measurements identify two sub-networks with the lower/higher one conveying BU/TD flow [Markov et al., 2014], but the two networks are tightly related.

The DM network is where response alternatives are activated to participate in competitions. When a focused response emerges upon competition resolution, most of the activated DM neurons are suppressed, but the ones lying on the focused path actively participate in the executing focused response. Hence, DM neurons participate in non-DM (execution) modes as well. This may be why some DM neurons project to the BG (both the dorsal and ventral STR), at least in mPFC [Gabbott et al., 2005].

\subsection{The adaptive response network}

Cortical L5 contains two types of pyramidal neurons [Harris and Shepherd, 2015]. The cortical response network consists of the L5 pyramidal neurons that project ipsilaterally to the thalamus, the BG, the spinal cord, other motor centers, and Rgen nuclei. Viewing them as response neurons is natural, since they are the only cortical neurons that project to innate motor centers. Response neurons generally reside in L5b and are thick-tufted, with dendrites that reach L1 and exhibit hyperpolarization-activated cyclic nucleotide-gated (HCN, subunit $\mathrm{HCH} 1$ ) cation currents (Ih), which support repeated firing at slow rates. They receive vertical input from the sensory network (both the thalamus and L4 neurons) and the prediction network, and provide vertical innervation to GABAergic (coordination) neurons, not to pyramidal neurons (see below). The response network is extensively internally connected across cortex (i.e., horizontally), but only ipsilaterally (see also nodes below). It sends horizontal long-distance connections through L1 to the DM network (see prediction below) and to apical dendrites of response neurons. The response network supports all adaptive responses, both learned and acute.

\subsection{The prediction (goals) network}

The L5 neurons that do not belong to the response network project to the other hemisphere, project bilaterally to the basal ganglia, and do not project to other subcortical structures. 
They are strongly inter-connected horizontally across cortex. They usually reside in L5a, some are tall and slender-tufted and some are short. Vertically, they are innervated by the DM network and innervate the response network (unidirectionally). We refer to them as the prediction network, because they represent the focused decision emerging from DM competition, but this decision is still not executed by the response network.

It may seem as if the prediction network is redundant, because the emerging decision could have been communicated directly from the DM network to the response network. However, this would immediately execute the response. The prediction network allows storing a competition winner without executing it, supporting intentions and predictions (see below). The DM and prediction networks are together called the internal or TD networks, and the response and prediction networks are together called the execution networks.

The execution networks send long-distance connections to the DM network, even though decision making precedes execution in the $\mathrm{R}$ process. This way, identified objects can influence the formation of higher level responses, and executing tasks can affect the interpretation of sensory inputs. Many such connections are from non-granular PFC areas to granular ones [Rempel-Clower and Barbas, 2000], i.e., from lower level areas first responding to alerts to higher level areas responsible for longer term responses. Such connections have a parallel function to that of the non-specific thalamic core, which conveys sensory flow to higher level areas.

\subsection{The thalamic TD network}

The thalamic core is the part of the BU sensory network that feeds inputs into cortex. The role of the more diffuse thalamic matrix is to sustain adaptive execution. Matrix neurons are innervated by the cortical response network (and possibly by L6 neurons as well) to convey TD flow. The matrix innervates the response and internal networks, especially the apical dendrites of L5 neurons, which extend to reach L1 (and the CCN, see below). When thalamic flow passes through the long narrow dendritic geometry above the cell's soma it is greatly amplified, supporting sustained operation [LaBerge, 2002 $]^{18}$.

In addition to its sustaining effect on individual neurons, the matrix also provides quax stability by triangulating intra-cortical flow and by synchrony, mostly via coordination networks (see below). Thus, the overall role of the thalamus is to provide energy drive to cortex, both BU and TD. Indeed, brain function critically depends on it [Ward, 2011].

\subsection{The basal ganglia (BG): gating execution}

Structure. The basal ganglia (BG) are a set of large bilateral abrain nuclei located at the center of the brain right above the thalamus [Graybiel and Mink, 2009] (see Figure 4). The flow between BG nuclei is largely directional, from input nuclei to output ones. There are three input nuclei: the dorsal striatum (dSTR), the ventral STR (or nucleus accumbens, NAc), and the subthalamic nucleus (STN). Output nuclei are the substantia nigra reticulata $(\mathrm{SNr})$ and internal globus pallidus (GPi), with a smaller output from the ventral pallidum (VP). There are internal nuclei, the external globus pallidus (GPe) and its ventral analogue the VP. The out-going neurons from all nuclei are GABAergic, except the STN's, which are glutamatergic. Those projecting from the STR are called medium spiny neurons (MSNs). BG neurons also project to Rgen (mostly DA and $\mathrm{ACh}$ ) nuclei.

\footnotetext{
${ }^{18}$ This may involve TD NMDA spikes [Major et al., 2013].
} 
The prediction and response networks (and more weakly also the DM network) in all cortical areas project to the STR. Motor areas project to the dSTR, and higher level task areas and valence areas project to the vSTR. There is substantial convergence along the cortex-STR-GPi/SNr direction [Bar-Gad et al., 2003]. Neurons in the output nuclei are tonically active [Wilson, 2015], and project to matrix neurons [Kaneko, 2013] in the non-specific nuclei of the thalamus innervated by frontal areas, and to subcortical motor centers (e.g., the SC). Cortico-STR projections are topographic, and this is preserved in the thalamus and in its projections onto cortex, giving rise to the well-known cortico-BGthalamocortical loops.

The BG nuclei are mutually and bidirectionally connected, but two main connectivity paths are evident: a STR-GPi/SNr path commonly called the direct path, and a STRGPe/VP-STN-GPi/SNr path called the indirect path. The MSNs participating in the two paths are generally molecularly different: the direct MSNs express dopamine D1 receptors, dynorphin and substance $\mathrm{P}$, while the indirect MSNs express D2 receptors and enkephalin. Both of the cortical execution networks project to both paths. There is neuronal overlap between the paths (e.g., one third of dSTR-GPe [Hegeman et al., 2016], and one half of NAc-VP projections, are from D1 neurons [Kupchik et al., 2015]).

Function. The tonic inhibition exerted by the BG output neurons on the non-specific thalamic nuclei prevents cortical quacia from recruiting thalamic matrix neurons and thereby prevents adaptive responses. To allow cortical recruitment of the thalamus, the BG output neurons must be either neutralized or coordinated. An important novel thesis of R17 is that the direct path neutralizes (disinhibits) the BG output neurons to support the competition $R$ mode, while the indirect path coordinates output neurons during responses (acute and automated). In other words, the direct path supports DM and its resulting focused decision, while the indirect path is trained by the direct path to execute the focused decision. As a result, the direct path is active only in the acute mode (mostly in its initial stages), while the indirect path is active in both acute and automated modes. Note that this account is dramatically different from the common view, especially with respect to the indirect path. The precise mechanism is discussed in depth under the DA and ACh modes below.

The STN is innervated by cortex ${ }^{19}$ (mostly by motor areas) and thalamus ipsilaterally, so we view it as part of the response network. Its main role is to provide excitatory drive to BG neurons to complement their inherent spiking. Indeed, suppression of the GPi and of the STN (e.g., by deep brain stimulation) is used to treat Parkinson's disease, in which impaired DA signaling does not allow the BG to function correctly. The two treatments are similar in that they both neutralize the BG, allowing cortex to recruit the thalamus directly. The STN also projects to the superficial cortical networks and disynaptically to the cerebellum, promoting rapid transitions to support response sequences (see below).

An additional important BG topic, the striosomes, is discussed under the consume $\mathrm{N}$ mode below.

In summary, the role of the BG is to control the formation and sustained execution of adaptive quacia, by gating cortical access to the thalamus and motor centers and by recruiting Rgens.

\subsection{The hippocampus}

The hippocampus, adjacent to the amygdala at the medial temporal lobe, is an abrain structure possessing a unique architecture [Buzsaki, 2006]. It is a rolled sheet whose long axis is

\footnotetext{
${ }^{19}$ This pathway is commonly called the hyperdirect path.
} 


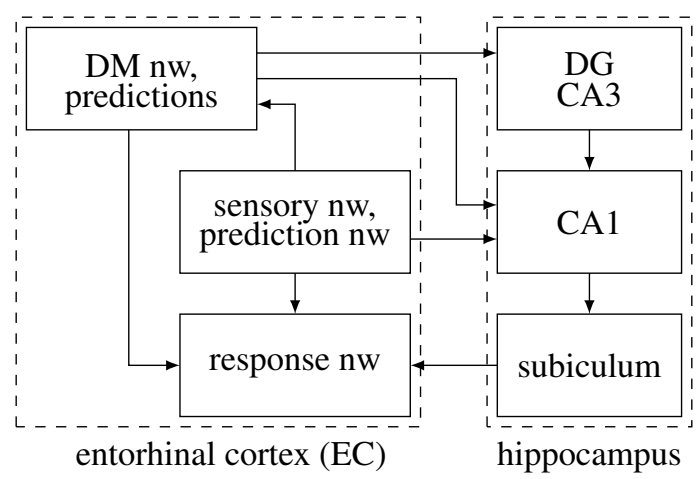

Figure 6: The hippocampus. The subiculum belongs to the response network. CA1 receives input from the EC prediction network (L3, since it is an agranular area), and from an automated L2 population that also represents predictions. Hence, CA1 belongs to the prediction network. The dentate gyrus (DG) and CA3 receive input from an EC DM (L2) population conveying acute flow, and belong to the DM network. To focus on the content networks, connections with thalamic and hypothalamic nuclei, the amygdala, BNST, the $\mathrm{BG}, \mathrm{PFC}$ and other areas are not shown. CA2 is not shown.

approximately dorsoposterior-ventroanterior and whose shorter axis is commonly divided into subfields. The main fields are the dentate gyrus (DG), CA3, CA2, and CA1, which are generally unidirectionally connected in this order. CA1 projects to an output structure, the subiculum. All fields except the DG have pyramidal content cells and coordination IINs as in cortex. Fields consist of three layers, the equivalents of L1, L5 and L6.

The hippocampus has attracted immense scientific and popular interest due to its role in memory [Moscovitch et al., 2016] and space [Buzsaki, 2006], but there is currently no coherent account for the roles of its subfields. We propose a novel account based on $\mathrm{R}$ process networks (Figure 6): (i) the DG and CA3 are part of the decision making network, (ii) CAl is part of the prediction network, and (iii) the subiculum is part of the response network. CA2 is smaller and may be specific for social interaction, combining both DM and prediction.

There are six main arguments supporting this account. First, the subiculum is the output field of the hippocampus, projecting to the EC execution network (L5), the STR (mostly projections from the ventral subiculum to the vSTR), PFC, etc. Second, cortical inputs to the hippocampus arrive mostly from the entorhinal cortex (EC). An EC L2 population that conveys acute DM flow projects to DG and CA3 [Kitamura et al., 2015]. Adult neurogenesis (NGNS) takes place in the DG [Christian et al., 2014], and in R17 the drive for NGNS is this DM network flow. Third, non-cortical inputs to the hippocampus are from the hypothalamic supramammillary nucleus (SUM) and the thalamic nucleus reuniens (NR) [Vertes, 2015]. SUM is connected with alert and innate areas (the septum, amygdala, PAG, VTA (DA), LC (NE)) and projects to the DG, CA3 and CA2, while NR conveys TD flow from mPFC to CA1 and the subiculum. Fourth, CA2 receives AVP inputs indicating acute needs, with emphasis on social needs and responses (see $\mathrm{N}$ process below). Fifth, the EC prediction network (L3, see agranular areas above) and an L2 population that participates in more automated actions (locomotion) project to CA1 [Kitamura et al., 2015]. Finally, learning (automaticity, and thus predictions) is reflected in CA1 more than in CA3 (it has many more place cells and they are more focused, see below).

Thus, we agree with previous observations viewing the hippocampus as a giant cortical column [Buzsaki, 2006], adding an R process view to elucidate the functional roles of the 
subfields. The hippocampus is further discussed under event areas and place cells below.

\subsection{Cognitive interpretation: predecision, reality, goals}

A brain theory must explain how cognition arises from the biological level. Here we provide a basic cognitive view of the roles of the flow networks, expanded under the $\mathrm{Q}$ process and internal cognition below.

During acute situations, the DM network is innervated by TD flow, its active neurons conveying many possible ways of perceiving the input and responding to it. In this situation, the DM network can be said to provide rapid non-specific (coarse) feedback (FB), and the sensory, alert and DM networks can be said to convey predecision flow ${ }^{20}$.

Situations having a high degree of uncertainty involve prolonged DM network activity, since certainty would lead to decision and focus. For example, the DM network would be used when expecting a relatively unimportant event whose timing is not known. In this function, we say that the DM network conveys general expectation or anticipation.

The DM network contains the neuronal candidate pool from which adaptive quacia emerge. As such, a wider DM network yields a wider diversity of responses. Thus, the DM network can be said to support cognitive flexibility and creativity. Indeed, the superficial networks are evolutionarily more recent than the execution networks, and their spine densities have considerably increased in primates, especially in humans [Elston et al., 2006].

The prediction and response networks convey focused postdecision flow resulting from competition resolution. Such flow is a prerequisite for perceiving an object or event, since perception (by definition) involves coherent entities and not an undefined flux or a simultaneous multitude of entities induced by the same sensory input. The response network is excited by sensory flow and is essential for generating external movements. Combining these observations, we posit that we perceive something as occurring in the external environment if and only if the response network in sensory cortices is activated ${ }^{21}$. For this reason, we refer to the response network as the reality network.

The prediction network is activated in acute situations, it is driven by the decision making network, it conveys focused flow, and its activation immediately precedes that of the response network. These data have two complementary cognitive interpretations. First, focused and effortful (acute driven) execution is what is usually meant by the term attention. Hence, we can refer to this network as the attention network. Second, when the focus network is activated but the response neurons it drives are still not activated, it represents responses activated before they occur in reality. Such responses are called predictions when referring to sensory events that are not controlled by the animal (e.g., 'this falling stone is going to hit the floor'), and goals, intentions or movement preparations when referring to sensory events controlled by the animal (e.g., 'my fingers are prepared to grasp this cup'). For brevity, we usually use only one of these terms, referring to this network as the prediction network. Although prediction may sound like an advanced cognitive function, the prediction network is a basic, evolutionarily ancient feature of the brain, and it is essential for executing actions and action sequences (see $\mathrm{Q}$ process below).

In summary of this section, brain anatomy strongly reflects the $\mathrm{R}$ process. Each $\mathrm{R}$ mode is associated with flow in a different neuronal network. Two prominent subcortical structures, the thalamus and basal ganglia, are involved in flow regulation and are essential for adaptive responses.

\footnotetext{
${ }^{20}$ Since the winning DM neurons also participate in focused execution, the DM network also exhibits postdecision activity.

${ }^{21}$ Conscious awareness is discussed under internal cognition below.
} 


\section{The Content Axis: Representations}

The cortical content dimension allows a large diversity of possible SR mappings by providing a rich space of representations. Here we present the node as the basic representation unit, and classify brain areas into object, action, valence and event areas.

\subsection{Representations}

We perceive the world as being comprised of different things (e.g., objects, materials, events). A perennial question about the brain is how it represents such things. R17 approaches this question from the perspective of the brain's basic execution unit, the sensorresponse pairing (the quax). We say that the brain represents an entity $E$ if and only if its responses to sensory inputs generated by $E$ are consistent and different from responses to other entities. As an example, consider a particular person's face. We know that the face is indeed represented only if it can arouse responses that are unique to it. There are several types of possible responses: familiarity ('I know this person'), recollection (recalling past events in which the person had participated), knowledge retrieval (recalling unique facts about that person, e.g., her name), predictions (how his voice would sound, what he is expected to say), change identification (e.g., a haircut, aging $)^{22}$, etc.

We can try to define the face's brain representation as the smallest set of neurons or connections without which none of these responses can be reliably made. However, there is no theoretical guarantee or experimental evidence that such a unique set exists, since different responses may utilize non-overlapping or only partially overlapping paths. At present, we can only define representations empirically using the best available measurement techniques, and these are mostly relevant to large areas rather than to individual neurons.

We say that an area or neuron $R$ represents content $C$ (a type of object, event, action etc.) if (i) $R$ is strongly activated when items of type $C$ are processed by the brain, and (ii) $R$ is only weakly activated when the brain does not process type $C$ items. A complementary definition requires that damage to $R$ impairs processing of type $C$ items ${ }^{23}$. Note that the third criterion is coarser than the first two (e.g., consider an impairment that affects all cortex). Note also that the fact that $R$ represents $C$ using these criteria does not mean that $C$ is the only content type that $R$ represents, or that $C$ is not represented by other areas/neurons as well.

The first two criteria can be measured in areas via fMRI, and in single neurons via direct recordings. However, since 'strong' and 'weak' are relative terms, the result depends on experimental protocol and other context details. Notably, a strong fMRI signal in an area reflects alert/DM network activity (see below), while representations are defined through the response network. In addition, whether and how sensory input is processed by the brain depends on the current R mode (e.g., the brain may ignore input that is not in the focus of attention or when it is irrelevant to acute needs). Moreover, for most types, any measurement can use only a small number of input items, so we must be careful when using broad labels to name types. The third criterion can be tested via pharmacological, electrical, genetic or injury-induced lesions, but it is also context-dependent, since 'impairment' is not a precise term.

\footnotetext{
${ }^{22}$ Note that some of these responses can be made through other inputs (e.g., the person's voice). This is immaterial to the issue of how the face is represented.

${ }^{23}$ Arguments from evolution can also be used to track areal representations, but these are complicated by the difficulty of establishing homologous areas between different species.
} 
These criteria allows us to say, for example, that the fusiform gyrus in the occipitotemporal lobe contains an area that represents faces. We can call this area the 'fusiform face area' (FFA) if convenient, but we must keep in mind two things. First, the processing of faces always involves neurons in other areas as well, and these can also be strongly active when processing faces even if they do not represent faces according to our criteria (i.e., in these areas the first criterion holds without the second). Second, nothing precludes the FFA from representing other types of content too. Indeed, there are compelling reasons to describe the FFA not as a face-specific area but as an area that represents objects that share a configuration of visual parts and whose acquisition requires expertise [Gauthier, 2017].

Because perception eventually results from the activation of the response network, we could define representation by using only the response network. This would make representations more context-independent.

\subsection{Nodes}

Many areas of cortex are organized in functional multi-neuron units that we call nodes. The defining property of a node is that it is a spatially focused vertical point of contact between the flow networks (see Figure 5). Anatomically, the node corresponds to the well known cortical minicolumn (MC) (sometimes called microcolumn) [Mountcastle, 1997]. MCs occupy a limited spatial extent (a few dozen micrometers), are roughly spatially distinct from other MCs, contain between a few dozen to a few hundred neurons, and span the full vertical dimension, containing all networks. There is high variability in all of the parameters of MCs, and some areas do not exhibit a clear MC organization. For our purposes, nodes and MCs are where the content networks interconnect most strongly, although they also interconnect across MCs.

As noted above, intranode internetwork connections are directional, the main patterns being sensory-response, alert-DM, DM-prediction, and prediction-response. Thus, the vertical flow direction inside a node is generally from the superficial to the deep networks. We say that a node is active in $\mathbf{R}$ mode $\mathbf{M}$ if and only if its $M$ network is substantially active and none of the networks of subsequent $\mathrm{R}$ modes are ${ }^{24}$. For example, a node is active in prediction mode if its prediction network is active but its response network is not.

Connections between MC neurons in the same flow network are very strong, so they are probably almost always excited and possibly activated together. Conversely, internode connections are not as strong, and since different nodes have different inputs there are situations in which one is active while the other is not. It is thus natural to surmise that node neurons in the same network represent the same thing ${ }^{25}$. Indeed, neurons in low level visual areas have narrow receptive fields and can be naturally viewed as representing the inputs that activate them. It is possible that this property extends to the response network of their minicolumns (but see [Horton and Adams, 2005]). However, our current measurement techniques do not allow us to conclude that specific objects are represented by specific networks of individual nodes.

I tend to believe that for many objects a unique representing set exists and is equal to the response neurons of a single cortical node, but whether this is true or not is an empirical question that we cannot answer at present.

It can be asked why a node has dozens of neurons in the same network if they are all

\footnotetext{
${ }^{24}$ Note that to apply this definition in practice, we need to know what levels of activation can be considered 'substantial'. This requires the creation of standard norms that may depend on area, task and species.

${ }^{25}$ Note that different networks of the same node represent different things. For example, DM network neurons in a given node are activated in more contexts than the node's response neurons.
} 
activated together. One answer is that this provides resilience against damage. Another answer is that different neurons may connect the node to different areas, to avoid excessive axonal branching. For example, it is possible that some response network neurons project subcortically while others project intracortically (but still ipsilaterally). .

Note that the existence of well-separated nodes and of discrete node representations is not essential for any aspect of R17, because all that matters is to resolve competition and reach a focused quax that maps sensory inputs to a response. Neither competition resolution nor response execution depend on separated nodes (see the Q process section).

\subsection{Object, action, valence areas}

The content type represented by an area is determined by its connectivity, in particular how close it is to input and output areas. Accordingly, we classify areas into three general types, object, valence and action, corresponding to the two main cortical input flow paths (sensory and innate) and the main output path (muscles). Object, action and valence areas are generally located in posterior-lateral, anterior and medial cortical areas, respectively.

Since the brain's basic processing and learning unit is the quax, which combines BU and TD flow, representations reflect both BU and TD connectivity. In particular, although direct connections with sensory inputs and motor outputs are major factors, even the organization of low level sensory and motor areas is strongly affected by task responses. This explains why cortical organization in animals that are congenitally impaired in a sensory modality is roughly similar to that in healthy ones, assuming that action areas are not impaired [Hahamy et al., 2017, Heimler et al., 2015].

Objects. Exteroceptive sensory inputs reach type-specific cortical areas known as primary sensory cortices. These include visual V1 at the occipital lobe, auditory A1 at the temporal lobe, somatosensory S1 at the parietal lobe, taste at the insula in the medial Sylvian fissure, and smell at piriform cortex in the deep temporal lobe. Proprioceptive paths reach somatosensory corte $\mathrm{x}^{26}$. Interoceptive paths continuously convey the state of the body, including mechanical, thermal, chemical, metabolic, and hormonal signals from the heart, gut, skin (the pain and pleasure aspects of touch), teeth etc. to the insula ${ }^{27}$ [Craig, 2014]. The capacity of rough estimation of time duration may rely on insular heartbeat representations [Wittmann, 2013] $]^{28}$.

Most sensory modalities also have higher level areas that are still mostly unimodal, called secondary sensory cortices. This includes vision (V2, V3, V4 and other occipital areas), audition (A2), touch (S2), and more anterior insular regions. Unimodal areas send inputs to multimodal object association (or integration) areas in the parietal and temporal lobes. Because motor inputs and outputs are generally dorsal, there is a distinction between dorsal areas, which are relatively context- and response-dependent, and ventral areas, which are relatively context-independent. The parietal lobe represents the part of space relevant for current actions, while the temporal lobe represents stable objects (e.g., faces, letters, words). The well known notion of the dorsal and ventral visual streams [Milner and Goodale, 2008] supports this distinction.

We collectively refer to these areas and to areas representing places (see events below) as object (or sensoriobject) areas.

\footnotetext{
${ }^{26}$ Since the proprioception area is separate from $\mathrm{S} 1$, a consistent notation would refer to it as 'primary proprioceptive cortex' and denote it as $\mathrm{P} 1$.

${ }^{27}$ Hence, a consistent notation would refer to these parts of the insula as 'primary interoceptive cortex' and denote them as I1.

${ }^{28}$ Precise timing of movements is a different capacity and relies on action automaticity.
} 
Actions. Areas generating TD flow are collectively called action (or response) areas. There are several sub-types. Motor areas are those involved in motor responses: motor cortex (M1), premotor cortex (PMC), and the supplementary motor cortex (SMA). Attention areas are those connecting to objects: dorsolateral PFC (dlPFC) and the frontal eye field (FEF). Task areas represent higher level and longer term aspects of tasks and planning, and include the more frontal part of PFC (the frontal pole, ventral PFC etc.). The language syntax areas are at the left (usually) inferior frontal gyrus (LIFG), extending across the Sylvian fissure (see language below). Naturally, there is overlap between these areas (e.g., it is natural to consider dlPFC as a task area too).

Note that object categories are distinguished both by shared sensory features and by the responses they arouse. Hence, categories are strongly represented in action areas, mostly PFC [Pan and Sakagami, 2012].

Action and object representations exhibit a center-edge gradient corresponding to response speed. Short term quacia involve centrally located (somatosensory, motor) nodes, intermediate quacia involve less central (parietal, premotor) nodes, and long term quacia involve edge located (PFC, temporal, occipitotemporal) nodes.

Valence. Valence areas represent the association between objects and innate responses, which have both motor and body-internal aspects. The cortical valence areas are mPFC, the insula, and orbitofrontal cortex (OFC). These areas receive motor aspects of valence from the amygdala and interoceptive body-internal aspects of valence from the thalamus. Note that the body-internal aspects of valence conveyed by interoceptive inputs are a direct reflection of the current $\mathrm{N}$ mode (e.g., the alert/execution modes increase/reduce heart rate, various agents convey information about hunger and satiety, etc.). Hence, cortex has direct access to $\mathrm{N}$ mode state and can utilize it during planning and decision making. Emotions are determined by $\mathrm{N}$ modes and can thus be viewed as a subtype of valence (see below).

Event valence is supported by the BNST and the hippocampus (below), especially its ventro-anterior part. The BNST, part of the extended amygdala, is a set of nuclei located roughly between the amygdala, the thalamus and $\mathrm{mPFC}$. The BNST receives inputs from the hippocampus, amygdala, VTA (a major DA nucleus) and lateral septum (LSep, a movement execution area), and sends output to the amygdala, ventral subiculum, dorsal raphe (a major SER nucleus), olfactory bulb, frontal cortex, hypothalamus, VTA, LSep, and NTS. The amygdala mediates between innate and adaptive areas, while the BNST is closer to the abrain, more strongly connected to place valence and less strongly to innates and objects.

mPFC receives direct visual, auditory, somatosensory [Liang et al., 2012] and interoceptive inputs from the thalamus, and innate+ flow from the extended amygdala. It is thus the cortical area first to identify need alerts (see claustrum below). In particular, it manages conditioned responses (see below). We designate $\mathrm{mPFC}$ as an area representing need or motivation, a subtype of valence. The insula and the ventral hippocampus are both sensoriobject areas and valence areas.

\subsection{Events, episodic memory}

The combination of an environment (place), objects, self movements, non-self movements and valence is called an event. Events are distinguished from the other data types represented by the brain in that the main area representing them, the hippocampus, has a unique architecture (see above) that supports adult neurogenesis ${ }^{29}$. Cortex is incapable of learning

\footnotetext{
${ }^{29}$ Adult neurogenesis occurs in three locations in the mammalian brain: the subgranular zone (SGZ) of the hippocampal dentate gyrus (DG), the subependymal or subventricular zone (SVZ) adjacent to the lateral
} 
events like it learns other data types, because the number of possible events is huge and most events are novel.

As described above, the hippocampus receives cortical inputs from the entorhinal cortex (EC) and is connected with valence areas via the hypothalamic SUM and the thalamic NR. In addition, hippocampus fields are mutually connected with the adjacent amygdala and the BNST. The ventral hippocampus represents valence more strongly than the dorsal hippocampus. The EC represents contextual scenes, places in its medial part (MEC) and objects in its lateral part (LEC). Learned places are represented at the parahippocampal place area (PPA) and the parasubiculum. Befitting their DM network role, CA3 neurons enjoy an enormous number of arbitrary interconnections [Buzsaki, 2006], hence are capable of supporting the representation of flexible event sequences. The hippocampus is not limited to spatial representations, as most CA1 neurons show sustained activity also for non-spatial events (sequential sound-odor combinations), including theta phase precession (see place cells below) [Terada et al., 2017]. Combining these data, we conclude that the hippocampus represents general valenced events and event sequences.

The subiculum is most strongly connected with two cortical areas. First, as indicated, it is connected with $\mathrm{mPFC}$ via NR, to integrate past and present events into the acute response. Second, it is connected with the retrosplenial cortex (RSC) and the precuneus, two medial areas in the posterior and superior parietal cortex (respectively). The subiculum and the adjacent presubiculum and parasubiculum are mutually connected with the RSC. The posterior parietal lobe (RSC and posterior cingulate cortices) is in turn reciprocally connected to the mPFC and parahippocampal cortex.

The capability of recalling events in which the self participates (personal events) is called episodic memory. There is substantial evidence, beyond the connectivity structure outlined above, that implicates an 'extended hippocampal system' comprising of the subiculum, anterior thalamic nuclei, mammillary bodies and RSC in human episodic memory [Aggleton, 2012]. It appears that the hippocampus is essential for initial event encoding, training the RSC (and to a weaker extent, the precuneus) to represent repeated episodic events (e.g., going to school, repeatedly recalled memories).

We refer to the EC, hippocampal formation, RSC and precuneus as event areas. When using a dichotomous distinction into objects and actions (e.g., as in SR mappings), events and places are grouped with objects, because we experience them as containing content (see awareness below).

Summary. In summary of the content dimension section, we can visualize cortical nodes as small dots densely populating the cortical sheet. Nodes and their inputs, outputs and interconnection patterns define the underlying quax space spanning the adaptive quacia that the brain can possibly form. Some of these dots correspond more closely than others to brain-external entities, but since the basic processing unit is the sensor-response mapping, it may not be possible to pinpoint exactly the dots representing specific entities. Moreover, even if a dot can be shown to represent a specific entity, it may also represent other entities. Nonetheless, cortical areas can be assigned broad representation types according to simple (but imprecise) criteria.

\section{The Q Process: Motor Control}

The Q process is the millisecond-scale process managing brain operation, including both movements and internal cognition (thought), by governing how quacia are formed and up-

ventricles, and the hypothalamus [Cheng, 2013]. 
dated. Specifically, the Q process supports the execution of hierarchical sequences. While the $\mathrm{N}$ process determines why things are done, the $\mathrm{Q}$ process determines how they are done. It utilizes synaptic neurotransmission, which is spatially and temporally focused, to allow rapid and precise communication. A major novel contribution of R17 is the detailed description of this process, which explains the implementation of motor actions, action sequences, action hierarchies, automaticity, attention, thinking, imagery, working memory, and the roles of prediction, anticipation, inhibitory interneurons (of various types), the cerebellum and the claustrum.

\subsection{Action goals, sequences \& hierarchy}

Goals. Consider the neurons involved in executing adaptive movements. It is natural to assume that action nodes (motor, premotor, PFC) active in reality mode are sufficient to drive such movements. R17 takes a different view, positing that execution also involves object nodes that specify the unmet sensory goals of the executed movement. Goals are sensoriobject configurations (e.g., fingers touching the surface of an object) represented by sensoriobject nodes. Since the movement's goal does not hold yet, the nodes are not active in reality mode but in prediction mode, or DM mode in case of uncertain goals.

The ibrain does not have a prediction network (but see imagery below). Innate movements are triggered by their hard-wired inputs and are guided by them (i.e., in a BU manner) and not by goals (TD). Cortical movements can be generated by action nodes alone, as in tics, but cognitively meaningful execution of adaptive actions always involves the specification of a goal ${ }^{30}$.

Action nodes innervate object goal nodes. Long-distance innervation of object prediction neurons is mostly done by action response and prediction neurons, but can also be done by action DM neurons. A major difficulty in learning to execute movements is that action nodes need to learn to activate the correct object nodes as goals. Indeed, infants' initial movements are not goal-directed, and they learn such movements slowly via exploration and feedback. At any age, learning of complex movements requires prior learning of simpler movements.

What we need to explain is what happens when goals are attained, how action sequences (as opposed to single actions) are performed, and how hierarchical action plans are implemented.

Goal attainment. Before a goal is met, reality neurons in the object nodes representing it receive TD input (from the node's prediction network, driven by the executing action nodes). When the goal occurs in reality, sensory inputs flow into the nodes representing it, so they now receive both TD and BU flow. Response neurons are large and generally cannot be activated by the relatively weak TD flow, but the BU flow activates them. Thus, the attainment of a goal involves the switching of nodes from prediction to reality mode.

Goal attainment does not always imply that the muscles driving the movement towards the goal should stop contracting. Commonly, the limb should remain at the goal position, which means that the non-isometric action during movement is replaced by an isometric action (i.e., it now involves the activation of both agonistic and antagonistic joint muscles). For example, when moving the fingers to grasp an object, the fingers continue exerting force after touching the object's boundary.

\footnotetext{
${ }^{30}$ Habitual learned actions are not triggered by motivational goals but by sensory inputs, but their execution does involve goal-setting.
} 
What activates the antagonistic muscle? The most elegant answer would be that it is driven by the newly activated object reality neurons. Indeed, object reality neurons have axons in the pyramidal tract projecting to innate motor circuits, and are thus capable of activating the antagonistic muscle. For example, stimulation of the BA5 area in the posterior parietal cortex (PPC) can drive finger and wrist movements, and BA5 reality neurons project to spinal interneurons that directly excite motoneurons [Rathelot et al., 2017]. Moreover, rodent S1 projects to spinal trigeminal nuclei and drives whisker retraction, while M1 projections drive whisker protraction [Matyas et al., 2010], providing direct evidence that object and action nodes can project to opposing muscles.

Hierarchical sequences: quax transitions. Most actions are part of hierarchical action plans. A dominant view is that action sequences are planned and managed in high level motor areas such as the SMA [Nachev et al., 2008]. Here we present a different view, in which sequences are not planned but emerge from sensorimotor interactions. In particular, the attainment of an action goal triggers the next action in the sequence.

To facilitate the subsequent discussion, we say that an action node $U$ mobilizes another node $W$ if neurons in $U$ participate in an active quax and innervate neurons in $W$ that are not part of the quax. Because neuronal interconnections require brain learning due to previously executed quacia, mobilization implies joint participation in past quacia. Mobilization can occur between two action nodes, not just between an action and an object node. Mobilization can occur in all networks. Initially, it is most pronounced in the internal networks, increasing in the reality network with automaticity. Thus, Q process competitions are held between mobilized nodes.

Suppose that a goal has been attained. Sensoriobject nodes representing it activate in reality mode. Besides innervating other reality nodes and motor centers, this activation generates sensory flow into action nodes via the thalamic BU network (reality neurons project to the non-specific nuclei of the thalamus, which pass this flow to higher level cortical areas). This sensory flow innervates the response, alert and DM networks in these action nodes. If neurons in one of these nodes are already excited to some degree, the arriving sensory input may activate them. A primary situation in which they are already excited is when they are mobilized by other action nodes, including higher level action nodes. In other words, if a goal has been attained, the resulting sensory input favors the activation of actions mobilized by currently executing actions. Thus, if an action was relevant in the past for attaining a goal set by currently executing actions (as reflected by mobilization by the latter), and if sensory preconditions for its execution occur, its triggering is facilitated. Crucially, this holds regardless of the number of other actions that are mobilized in parallel by the same high level action node.

This process explains how the next action in an action sequence is selected and how hierarchical action plans are implemented. The resulting account is quite simple: (i) higher level actions mobilize all of the concrete goals and lower level actions that have served them in the past; (ii) the next lower level action is selected by sensory inputs representing the attained goal of the current action ${ }^{31}$. Higher level action nodes do not need to plan a sequence or determine the order of execution of lower level actions. All they need to do is mobilize their past goals and auxiliary actions (i.e., excite candidates in a DM competition mode), and the external context determines the actual sequence. The current sensory context selects the goal $G$ that best matches external reality, and $G$ selects the next action ${ }^{32}$.

The above explains all levels of the execution hierarchy. As an example using a crude

\footnotetext{
${ }^{31}$ Selection is also supported by a GABA-based competition mechanism, see below.

${ }^{32}$ Obviously, mobilization is not the only factor that determines the next action, since many factors mold the neural terrain, including recent execution, Rgens reflecting current $\mathrm{N}$ mode and needs, object valence, etc.
} 
division of action nodes into need, task and motor nodes, consider active high level need neurons conveying the message 'movements should be performed to satisfy need Z' (e.g., hunger). This drives task neurons that convey the message 'movements should be performed to attain goal $\mathrm{Y}^{\prime}$ (where $\mathrm{Y}$ is a specific way to satisfy $\mathrm{Z}$, e.g., eating this particular apple to satisfy hunger). These neurons drive motor action neurons that implement a concrete movement sequence, where each such neuron conveys 'produce movement $\mathrm{X}$ to satisfy an intermediate goal G' (e.g., reach with the arm until it is close to the apple, move the fingers until they grasp the apple, move the arm back until the apple touches the lips, etc.). As long as the task neurons produce TD flow, the sensory context determines which small movements are actually executed and their order of execution (here, that the finger grasping movements start executing only when the hand gets close to the apple).

Action termination. Some actions whose goals have been attained should stop driving execution. There are three mechanisms involved in action quenching. The first involves competition. Muscle contraction should be terminated mostly when starting non-isometric actions that involve the antagonistic muscle. In this case, antagonistic motor neurons suppress the agonistic ones, which is a simple form of competition. Similarly, when nodes representing the attained goal select the next action, it joins the quax to start executing. The incorporation of a new node into a quax involves competition (see below), which may result in the suppression of excited actions.

Second, when action nodes lose their drive, their neurons' firing rates decrease and they naturally terminate. Higher level action nodes drive gMTNs, and when their activity is reduced, proprioceptive sensory input decreases, thereby depriving M1 neurons of a large part of their drive. This conforms with the view of gMTNs as being the innate part of the prediction network (see imagery below).

A third putative mechanism involves Rgens. All cortical response neurons project to dopamine (DA) nuclei. When a goal is attained, it moves from prediction to response mode, causing DA release. This release is topographic, reaching the node itself and the BG paths supporting it. If the action is automated, the amount of DA released is small, causing suppression of the execution paths via D2Rs ${ }^{33}$. Other Rgen receptors (alpha2 NERs, nicotinic AChR, some serotonin receptors) may play a similar role, see their sections below.

Supporting evidence. Most aspects of the Q process are supported by evidence. A large body of evidence for general predictions in the brain is reviewed in [Clark, 2015]. Sensory and motor areas are clearly tightly linked. For example, M1 and S2 (not just S1) have mutual projections across all layers [Suter and Shepherd, 2015]. Sensory areas are essential for correct movements, as shown by parietal lesions and temporary disruption by TMS [Davare et al., 2015]. Multiple motor alternatives are prepared in parallel [Cisek and Pastor-Bernier, 2014], supporting our notion of mobilization. Motor areas are organized not only according to the limb used (the classical body map), but also according to the sensory requirements of the action [Graziano, 2016]. In other words, motor nodes that have similar sensory preconditions and goals are adjacent to each other, which in our view occurs because they are commonly mobilized together by object and other action nodes.

[Desmurget et al., 2009] provide strong support for the $\mathrm{Q}$ process, in particular for action termination being implemented by object node activation in reality mode. In people with parietal lesions, weak stimulation of inferior parietal areas (presumably activating the internal networks, setting movement goals) created a feeling of intention or desire to move the corresponding organ, while strong stimulation (presumably activating the response network) resulted in a feeling of having actually moved. Stimulation of motor areas resulted

\footnotetext{
${ }^{33} \mathrm{D} 2 \mathrm{Rs}$ can also yield relaxed oscillations (see DA), supporting continued activity of higher level nodes.
} 
in movement but patients denied having moved. That is, self-reporting of movement is not based on motor nodes, but on object nodes being activated in reality mode (see also awareness below).

The R17 account is close in spirit to the old notions of affordances and the ideomotor principle and to their modern versions (e.g., the affordance competition hypothesis [Cisek and Pastor-Bernier, 2014] and the theory of event coding [Hommel, 2009]). Virtually all of the substantial evidence supporting these notions (see these references) supports our account as well. Unlike these theories, the R17 account is mechanistically detailed (the roles of the various networks, competition, transitions), and is integrated with other events taking place during the $\mathrm{Q}$ process (surprises, coordination etc). In addition, it is different from these theories in some important points (the role of the BG). Additional evidence for the $\mathrm{Q}$ process (EEG, repetition suppression, efference copy, etc.) is given below.

\subsection{Coordination: competition, synchrony, initiation}

Competition. Alerts generate BU flow in the alert network, which excites its node's DM network to yield TD DM flow. The existence of a surprising alert means that there is no adaptive response that is specific to the alerting event. Instead, many relevant adaptive responses are mobilized, and compete to be included in the emerging flexible response. Relevance is determined by the reach of alert flow, a function of the present context (the nodes already excited when the alert arrives) and past responses (existing UQS connections). In particular, nodes representing responses to events that share some feature with the current one are excited.

The mechanism that implements the selection of a focused response from the candidate pool is called competition. Competition and subsequent sustained quax execution via synchrony are mediated by brain-wide coordination networks consisting of GABAergic inhibitory interneurons (IINs) (i.e., locally projecting neurons). IINs sometimes also participate in quax initiation. Hence, the role of GABA in R17 is to support effortful execution, contrary to the standard descriptions viewing it as inhibiting execution ${ }^{34}$.

JOS. Coordination utilizes an IIN-mediated join-or-stop (JOS) mechanism that works as follows. Suppose that two neurons have similar physiological characteristics and are targets of the same oscillating inhibitory IIN. If their energy drives are of comparable magnitudes (e.g., when they represent the same thing), and if the IIN's firing rate is not faster than their charging rate, the IIN's inhibition synchronizes their firing (it resets their electric charges, allowing them to start charging at the same time. Due to their comparable charging rate, they then fire at the same time). We refer to this as a join operation. If one neuron is supported by a strong drive while the other is not, the IIN would stop (silence) the weakly driven one. If the IIN fires faster than the two charging rates, it would silence both neurons. Thus, the effect of activated IINs is to magnify signal (defined as being in synch with the IIN) and quench noise. We say that two excitatory neurons engage in a competition if an IIN needs more than one firing to affect JOS.

Any innervation of an IIN by an excitatory neuron can be viewed as a coordination request. Coordination neurons are driven by excitatory network flow, both locally and over long-distance connections. When flow enters an area and activates its excitatory neurons, these quickly activate its coordination networks, to separate the area's excitatory neurons into those according with the flow (join, defining winners) and those not according with it

\footnotetext{
${ }^{34}$ Large exogenous amounts of GABA can inhibit execution by taking advantage of its mechanistic action, but this is not GABA's physiological role.
} 
(stop, defining losers). Thus, JOS is an arbitration mechanism between neurons for getting included in the quax. After competition resolution, JOS sustains local synchrony between the winners. Synchrony is propagated because single IINs innervate many content neurons, and because IINs are connected by gap junctions, which facilitate synchronized firing (see below).

We refer to the IINs active in a quax as its scaffold, and to its content and Rgen neurons as its skeleton. The skeleton determines the currently executing actions, the object(s) that they act upon, and why they are executed. The scaffold maintains skeleton neurons in local synchrony and protects from interference from other excitatory neurons. The skeleton uses glut in the abrain, and glut and acetylcholine (ACh) in the ibrain. The scaffold uses GABA in the abrain, and glycine and GABA in the ibrain ${ }^{35}$.

Substantial evidence supports the role of GABA in competition resolution. Particularly convincing are the glut and GABA uncaging experiments described in [Hayama et al., 2013], where GABA induced widespread spine shrinkage (a short term structural plasticity phenomenon reflecting local loss of competition) across the dendrite, except at the spine belonging to the quax skeleton.

Disinhibition. To prevent erroneous responses, responses are suppressed by default throughout the brain. In several key junctions, this is done by tonically active inhibitory neurons. Elsewhere, it is done via a general ambient inhibitory tone. In the DM mode, many different response alternatives should be allowed to compete, and this is achieved by inhibition of the default inhibition. In other words, competition involves the opening of a wide disinhibitory constraint-free path (a 'hole') in the default inhibition. After competition resolution, only the winning alternative keeps being allowed (disinhibited), and the default inhibition of the losers is reinstigated ${ }^{36}$.

The main tonically active disinhibitory junctions are at the basal ganglia (BG)-thalamus, cerebellar cortex-output nuclei, PAG-medulla (innates), and BG-superior colliculus (eye movements $)^{37}$. The main non-tonically active disinhibition is in the competition coordination network $(\mathrm{CCN})$, see below.

Automaticity. Repeated task training that exposes the brain to a rich variety of input combinations leads to a UQS that provides good BU flow separation, whereby neurons on the correct response path receive high flow while potential competitors receive weak flow. In this situation, a response can be found in a single BU pass, with minimal TD flow (mainly consisting of precise predictions) and competition. Thus, automaticity involves BU triggering and reduced alerts and TD control ${ }^{38}$. Conversely, acute events require TD flow to resolve competitions.

If the neurons about to win a competition are already partially excited before sensory input arrives, competition is resolved faster. There are two common cases in which this occurs, repeated immediate execution of the same quax (see repetition suppression below) and anticipations and predictions that match the input. Improved prediction accuracy is another consequence of repeated training on a task, since quax transitions are repeated with training and allow learning processes to refine the UQS.

\footnotetext{
${ }^{35}$ The skeleton and scaffold are also supported by additional Rgens, see below.

${ }^{36}$ Tonically active inhibition is also useful for enabling rapid responses, because excitation of the response neurons can start before the response should be executed (since it is inhibited). That is, relatively complete responses can be prepared before competition is even seriously started, without risking premature execution.

${ }^{37}$ The retina also uses disinhibition (BU), at least in some species.

${ }^{38}$ Note that it is difficult to execute automatic routines in attended TD mode (e.g., try to tie your shoelaces slowly with attention). With automated tasks, the brain's connections are optimized for BU flow, so forcing the usage of TD flow disrupts execution.
} 
Initiation via GABA. GABA is the brain's main inhibitory agent. Nonetheless, it can be excitatory under certain conditions. GABA works through two families of receptors, ionotropic GABAA receptors, which act quickly through ion channels, and metabotropic GABAB receptors that induce slower effects [Benarroch, 2012]. GABAARs are comprised of five subunits of various isoforms. Synaptic GABAARs mainly contain the alpha1 or alpha2 isoforms, and extrasynaptic GABAARs mostly contain the delta isoform. When they bind to GABA, GABAARs induce the opening Cl- channels. Flow direction depends on the $\mathrm{Cl}$ concentration gradient between the cell's inside and outside. If the intracellular concentration is higher, GABAAR binding results in $\mathrm{Cl}$ - outflow, a situation called excitatory (depolarizing) GABA.

Early in development, the transporter NKCC1 continuously moves $\mathrm{Cl}$ - into the cell, resulting in excitatory GABA. During development, $\mathrm{NKCC1}$ is downregulated and the potassium-chloride cotransporter $\mathrm{KCC} 2$, which moves $\mathrm{Cl}$ - out of the cell, is upregulated [Ben-Ari, 2014]. In the common adult state, the $\mathrm{Cl}$ - equilibrium potential is more negative than the resting membrane potential. In this case, GABAAR binding lets $\mathrm{Cl}$ - into the cell and GABA is hyperpolarizing. However, depolarizing GABA is not limited to early development, and occurs in the adult state after initial strong stimulation, e.g., in the retina [Lindstrom et al., 2010], hypothalamus [Bains, 2014], spinal cord, amygdala, cerebellum, and hippocampus [Marty and Llano, 2005]. Acute situations yield excitatory GABA by downregulating KCC2 via BDNF (a structural plasticity agent released during acute responses) [Rivera et al., 2004] and/or by alpha1 NERs indicating alert [Bains, 2014].

Thus, at least in some cases, GABAergic IINs induce quax initiation, providing neurons with an initial excitatory push after surprising or strong sensory input. GABA-mediated coordination includes quax initiation, quax formation by disinhibition and competition, and sustained quax execution via synchrony.

\subsection{Coordination networks}

GABAergic IINs (reviewed in [Tremblay et al., 2016]) are ubiquitous in all brain areas, comprising around $15-20 \%$ of the neurons in cortex ${ }^{39}$. Inhibitory neurons are commonly classified according to many dimensions, including excitation speed, cortical layer, innervated target compartment, morphology, and protein expression [DeFelipe et al., 2013]. R17 introduces a novel R process-based classification of IINs into coordination networks (CNs) (Figure 5).

When flow activates excitatory neurons, they request coordination by innervating IINs. Since the different $\mathrm{R}$ modes differ in flow characteristics, there are several different CNs generally active in different $\mathrm{R}$ modes. Consequently, IINs in the same $\mathrm{CN}$ need to be synchronized, but inter-CN synchronization is not needed. Indeed, all of the IIN classes (except VIPs, see below) are internally connected at varying degrees by both electrical (gap junction) and chemical synapses, while electrical connections between the classes are not common [Hestrin and Galarreta, 2005].

The innate coordination network (ICN). The ICN consists of IINs in the spinal cord, pons, medulla, PAG and other innate centers. In the motor unit, sensory neurons innervate IINs targeting antagonistic muscles (in addition to agonistic aMTNs), to allow competition between the two possible limb movement directions ${ }^{40}$.

\footnotetext{
${ }^{39}$ It is to be expected that there would be fewer coordination than content neurons, because a single IIN should coordinate several content neurons.

${ }^{40}$ Isometric force uses both muscles, and involves tremor even in healthy animals due to IIN-mediated competition.
} 
The execution coordination network (ECN). The ECN includes two IIN groups, both basket (soma-targeting) cells. The first consists of parvalbumin (PV)-expressing basket IINs. These IINs are present in L2-6, target pyramidal somas mostly in the deep networks, receive strong sensory network flow, and are fast-spiking (FS). PV IINs have calciumpermeable $(\mathrm{CP})$ glut AMPA receptors, supporting rapid excitation and spiking. Somatic innervation is the best way to achieve cell-wide JOS. PV binds Ca2+ with high affinity to allow fast repeated excitation, and its ability to do so is maximal at gamma frequency (30$80 \mathrm{~Hz}$ ) [Henzl, 2013]. Thus, these PV IINs are capable of coordinating the high frequency pulsing exhibited during response execution.

The second group consists of basket IINs that express cholecystokinin (CCK) (many CCKs also express calbindin, and some express PV). The CCKs target excitatory L2/3/4 neurons, i.e., the neurons involved in predecision (and later, focused) acute responses, and express presynaptic $\mathrm{CB} 1$ receptors used by endocannabinoids (ECBs) to suppress presynaptic activity and terminate the acute response (see the resolution $\mathrm{N}$ mode below). Postsynaptically, CCK synapses express nicotinic ACh receptors and GABAARs enriched in the alpha2 subunit, both associated with acute focused flow (see below). Thus, the two basket IIN groups clearly support sustained synchrony during execution. Indeed, L4 PVs receive stronger thalamocortical inputs than their neighboring excitatory cells. Reducing the activity of the thalamic nucleus innervating PFC, the mediodorsal (MD) nucleus, mostly reduces GABAergic signaling, specifically in PV IINs [Ferguson and Gao, 2017], showing that the thalamus and the ECN cooperate in supporting sustained execution.

The competition coordination network (CCN). The CCN consists of three IIN populations, one inhibiting responses, another disinhibiting the first, and a third constraining the second. The first group contains Martinotti IINs, which express somatostatin (SOM), target apical dendrites of L5 and possibly L2/3 pyramidal neurons, and are innervated by L5 neurons. The activation of SOMs excludes synaptic inputs from the quax, implementing the stop part of JOS to narrow the execution path. SOM is also known as growth hormoneinhibiting hormone. Since the plasticity effect of competition is growth, this accords with SOM neurons limiting competition ${ }^{41}$.

The second CCN population consists of L1-3 IINs expressing vasoactive intestinal polypeptide (VIP), nicotinic ACh receptors, the ionotropic serotonin receptor SER3, and calretinin. These IINs are unique in that they mostly target other IINs rather than pyramidal neurons. They suppress the SOM-expressing IINs, and are innervated by long-distance cortical connections (e.g., from action to object nodes). The VIPs support competition and the subsequent winning path by suppressing the SOMs that suppress this path, thereby disinhibiting the response network [Karnani et al., 2016, Letzkus et al., 2015]. In V1, VIP IINs innervated by long distance frontal connections enhance receptive field centers [Zhang et al., 2014]. The VIPs are the only IIN class not internally connected by gap junctions, supporting a role in focused execution ${ }^{42}$. VIP is a powerful vasodilator peptide, supporting its role in the acute response.

The third CCN population consists of neurogliaform cells (NGFCs). The main NGFC population is located in L1, and expresses SER3 (like the VIPs) [Rudy et al., 2011]. These IINs are innervated by the thalamic TD network and target pyramidal apical dendrites and

\footnotetext{
${ }^{41}$ A recently discovered IIN group includes SOM IINs in L4, L5b and L6 that target the execution (deep) layers and support BU flow [Muñoz et al., 2017]. These IINs may support the stop function of JOS for automated execution, but more information is needed to reach firm conclusions.

${ }^{42}$ Note that the usage of two consecutive GABAergic connections does not pose any problem to the quax, because a GABAergic neuron can coordinate another GABAergic neuron in the same way that it coordinates excitatory neurons, as long as both receive excitatory input.
} 
all IIN types. There are elongated L1 NGFCs that span several columns [Jiang et al., 2013]. Another NGFCs population is located in L4 and is innervated by the thalamic BU network. NGFCs provide diffuse and prolonged inhibition, mostly targeting extrasynaptic delta GABAARs and the high affinity GABABRs. They thus seem to provide prolonged silencing of competition losers ${ }^{43}$. Winners are allowed by the execution Rgen ACh, which suppresses L1 NGFCs [Brombas et al., 2014] (see below). Note that both NGFC groups are innervated by the thalamus, according with its role of sustaining execution, here by preserving competition results.

The response suppression network (RSN). A fourth coordination network consists of chandelier IINs, which target the axon initial segment (AIS) of pyramidal neurons [Fish et al., 2013], which is where action potentials are triggered. They express PV, are mostly present in PFC, suppress the node's internal (TD) networks, and are electrically interconnected with each other. They mostly activate alpha2 GABAARs, which are associated with early development and acute events, and their axon cartridge density decreases after adolescence. Long-term synapse density decreases during automaticity, since it induces learning processes that are opposite to those induced by acute growth. Thus, this network seems to learn a TD response to acute situations that matures during adolescence, a response that rapidly and strongly suppresses internal network cell firing. As a result, it can be viewed as a response suppression network.

Chandelier synapses are adjacent to postsynaptic SER1a receptors, most of which are located on the AIS and suppress responses as part of the mistake $\mathrm{N}$ mode (see habenula, SER below). The RSN may induce initial excitation of silent neurons [Woodruff et al., 2009, Blot and Barbour, 2014], supporting its participation in acute situations (since this is the main role of excitatory GABA). Unlike the other coordination networks, this network may support only the silencing part of JOS, because neutralizing the AIS suppresses neuronal firing.

Coordination networks exist in all brain areas. In particular, the amygdala, BG, and hippocampus have PV and CCK/CB1 basket IINs and SOM IINs, whose roles are generally as in cortex. The thalamus has local coordination, and it is also surrounded by a thin layer of GABAergic neurons innervated by L6, the thalamic reticular nucleus (TRN), which comprises the cortico-thalamic coordination network.

\subsection{ERPs, repetition suppression, predictions}

In addition to the specific supporting evidence cited above, there are several basic brain phenomena supporting our Q process account.

Event-related potentials (ERPs). Cortical pyramidal neurons have a main dendritic branch oriented perpendicular to the cortical sheet. Electroencephalography (EEG) is a measurement modality in which voltage changes are sensed using electrodes placed on the surface of the skull, thus mostly reflecting the relative distribution of neuronal activity between the superficial and deep layers [Kotchoubey, 2006]. When EEG measurements are synchronized with behavioral responses and averaged to reduce noise, clear negativities and positivities called event-related potentials (ERPs) are obtained.

ERPs are usually denoted by their timing relative to an event (usually, stimulus appearance) or their ordinal number after the event, and sometimes by behavioral phenomena or experimental paradigms. Well known ERPs include the N170 (face recognition), MMN

\footnotetext{
${ }^{43}$ In L4, they also silence L4 FS IINs, most likely those recruited by losers.
} 
(mismatch negativity, usually auditory), N2 (visual attention), ERN (error-related negativity), P3b (also P300, frontal response to an object in object sequences), ELAN (early left-anterior negativity, local language syntax), N400 (response to words), and LDAEP (loudness dependent auditory evoked potential).

ERPs provide strong evidence for the R17 competition process and the role of the superficial and deep networks. Negative ERPs reflect the peak of DM network activity, while positive ERPs correspond to the focusing of the response after competition resolution. Indeed, ERP negativity size solidly correlates with the amount of surprise. For example, a larger N400 reflects a larger semantic surprise (see language below). Larger positivities reflect increased competition, as seen in the P3b component, which is larger for rare stimuli [Verleger, 2008]. This supports the role of learned anticipations in reducing alert and DM responses.

The presentation of a stimulus usually induces several ERPs. Each ERP may correspond to a thalamo-cortical wave, since thalamic flow is strong and its arrival promotes competition resolution. In this case, each thalamic nucleus would have an associated ERP in a given specific task.

Repetition suppression (RS). RS (or adaptation) refers to the empirical finding that if an event is repeated several times, its measurable effects are decreased. This phenomenon supports the R17 Q process, because when an event is repeated the acute response to it is smaller (see alert mode below).

RS experiments commonly use functional magnetic resonance imaging (fMRI), a highly popular modality based on brain blood-oxygen-level dependent (BOLD) contrasts. The brain is divided into three-dimensional areas (e.g., 1 millimeter ${ }^{3}$ cubes), and BOLD values in each area during two states (usually before and after some event) are recorded. Unlike EEG, fMRI is temporally imprecise, reflecting changes taking place across seconds and more $^{44}$. Spatially, it is much more precise than EEG, but is still highly imprecise since it groups together the activity of a very large number of cells. BOLD data provides strong support for the assignment of representation content to brain areas. BOLD mostly reflects TD flow [Cardoso et al., 2012], showing clear repetition suppression following repeated stimuli.

Another measure that shows RS is prepulse inhibition (PPI), where a strong stimulus induces a weaker startle reflex when presented after a weak stimulus. The fact that there are TD influences on the PPI [Li et al., 2009] shows that the existing neuronal terrain can affect innate circuits, not just abrain ones.

Efference copy. There is a large body of evidence for so-called sensory predictions during the execution of movement. A lot of it is associated with the classical notions of efference copy and corollary discharge, which emphasize the distinction between the consequences of self-movement and other external changes [Poulet and Hedwig, 2007]. However, careful examination shows that this kind of evidence supports the R17 goaling account. Particularly impressive are the results reported in [Niziolek et al., 2013], where magnetoencephalography (MEG) in human speakers showed that efference copy does not track movement variability across repetitions of the same motor task, and hence seems to represent the sensory goal, not a motor command copy.

\footnotetext{
${ }^{44}$ Although there are modern methods that can greatly improve accuracy.
} 


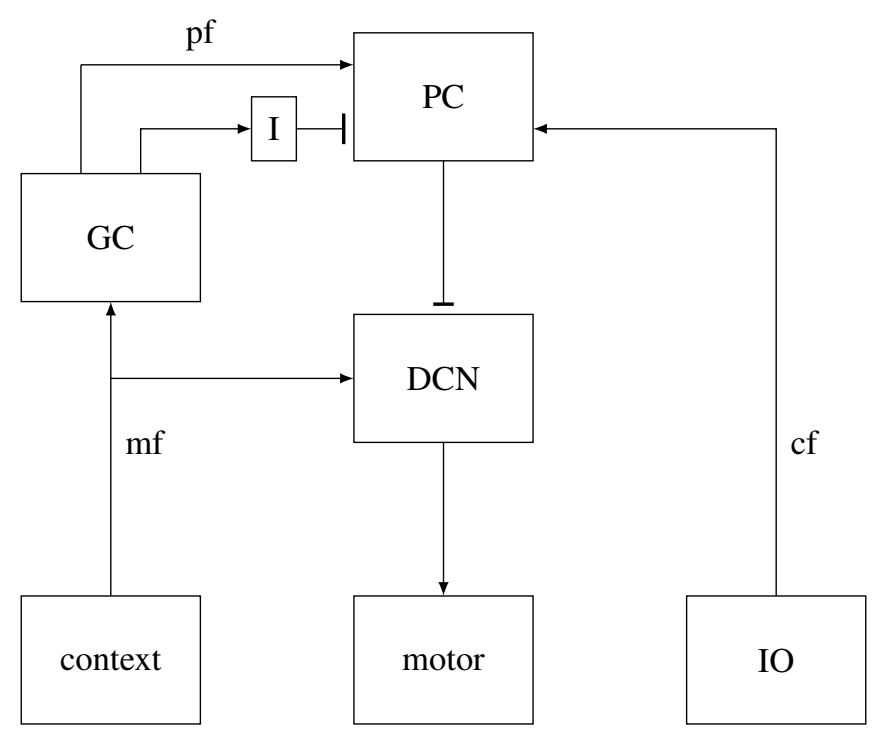

Figure 7: The cerebellum. Purkinje cells (PCs) tonically suppress the deep cerebellar nuclei (DCN), which excite motor centers to execute responses. Sensory input to PCs arrives from the inferior olivary (IO) through climbing fibers (cf), innervating PC somas and proximal dendrites. Contextual inputs from the pons, cortex etc. arrive via the mossy fibers (mf), exciting granule cells (GCs) and the DCN. GCs excite PC apical dendrites and local inhibitory interneurons (I), which suppress PCs. When BU (cf) and TD (mf) PC inputs coincide, the $\mathrm{PC}$ is briefly suppressed to disinhibit its response. Only the main connections are shown (e.g., IO-DCN and IO-GC connections, the various IIN types, and suppressive DCN-IO connections are not shown).

\subsection{The cerebellum}

The cerebellum (CER) (Figure 7) is a uniquely structured part of the brain, comprising the hindbrain with the medulla and the pontine nuclei. Its principles of operation are generally understood. Here we describe an $\mathrm{R}$ process perspective that provides a clear picture.

Neurons in the CER output nuclei (the deep cerebellar nuclei, the largest of which is the dentate nucleus) innervate motor centers, where many output neurons directly contact motoneurons. With the closely related vestibular nuclei, CER output nuclei also access spinal circuits via reticulo-, vesticulo-, tecto- and rubro-spinal connections. Thus, CER output neurons are part of the response network. CER flow also reaches cortex through the thalamus, with a substantial part of CER flow not being gated by the BG. CER flow projects to the sensory and prediction networks [Kawaguchi, 2017], mainly in motor areas (M1, SMA) and the parietal cortex.

Cerebellar (and vestibular nuclei) output neurons are innervated by Purkinje cells (PCs) in the cerebellar cortex, which are GABAergic and tonically active at high frequencies, constituting a disinhibition junction. PCs receive two types of glut inputs, parallel fibers from cerebellar cortex granule cells and climbing fibers from the inferior olivary nucleus (IO, part of the medulla). There is a vast number (dozens of billions) of granule cells, which target PC dendrites at substantial convergence (each PC is contacted by axons from $200 \mathrm{~K}$ $1 \mathrm{M}$ granule cells). Granule cells receive inputs from many sources, mostly from cortex via the pontocerebellar pathway but also from the spinal cord and brainstem nuclei. They also receive feedback from the deep cerebellar nuclei. Granule cells innervate stellate and basket IINs that target PC somas and axon initial segments using ultra-fast ephaptic action 
[Blot and Barbour, 2014]. The CER also has coordination networks, for example Golgi IINs that targets granule cell dendrites.

The IO climbing fibers receive BU sensory input, mainly proprioceptive, vestibular and visual, and innate activation information from the PAG [Koutsikou et al., 2014]. Climbing fibers target Purkinje cell bodies and proximal dendrites, according with conveying BU inputs. Each PC receives input from only a single climbing fiber, and each climbing fiber contacts just a few (1-10) PCs.

As usual, both TD and BU (parallel and climbing fibers) inputs are needed to activate granule cells [Jörntell, 2017] to disinhibit their target PCs [Heiney et al., 2014, Ishikawa et al., 2014]. PC disinhibition is possibly done by granule cell activation that suppresses PCs before it excites them [Blot and Barbour, 2014]. In this view, parallel fiber excitation of PC dendrites supports action suppression (and thus action sequences) rather than action release. In addition to PC inputs, the deep cerebellum output neurons receive the same type of excitatory inputs as PCs (IO and mossy fiber inputs, which are collateral to granule cells), which supports rapid disinhibition. Additional research is needed in order to elucidate the precise disinhibition mechanism.

Taken together, we conclude that the role of the cerebellum is to generate rapid adaptive responses, with an emphasis on transitions (i.e., action sequences). Granule cells are part of the prediction network. Each granule cell represents a detailed learned contextual state. There is a vast number of granule cells because the predictive state space is very large. Transitions (PC activations) are triggered by highly specific sensory inputs arriving via the climbing fibers at short latencies. There is a smaller number of PCs than granule cells because the sensory input space is smaller than the context space. Since a lot of CER flow is not gated by the thalamus, it can reach cortical motor areas very quickly to induce low(M1) and higher-level (SMA) transitions. Since the CER implements adaptive responses and all adaptive responses are allowed by the BG, the CER has reciprocal connections with the BG, mostly with the STN, which supports learned responses [Bostan et al., 2013].

A large body of evidence supports the involvement of the cerebellum in fast predictions (e.g., [Schlerf et al., 2012]). PCs have collaterals that target the cerebellar cortex, including granule cells, allowing the cerebellum to support action sequences [Guo et al., 2016]. Granule cells learn to activate after the stimulus and before the response [Giovannucci et al., 2017]. Language requires fast transitions due to the relatively high speed of speech articulation. The cortico-CER-cortical loops include non-motor loops that also support language and high level task areas [Bostan et al., 2013].

\subsection{Attention: orienting, the claustrum}

Orienting of attention (OOA). There is a special innate $Q$ process that continuously occurs during wakefulness in many species, called the orienting of attention (OOA). OOA is a set of innate actions that locate sensory organs to bring more information about the stimulus and enable the animal to better confront it. OOA includes saccades (moving the fovea) and head, trunk and general body posture movements. The main OOA centers are the superior colliculus (SC) and the inferior colliculus (IC), which focus on visual and auditory inputs respectively. Visual input innervates superficial SC layers, and it has auditory and tactile inputs at the intermediate layers. The IC has mostly auditory inputs, and its acute responses are through projections to the SC.

Input reaches the SC directly from the retina and from the thalamus. If it is surprising, it triggers a saccade whose goal is to have the fovea aligned in that direction. Saccades are constantly triggered by small visual surprises. Normally, there are 2-4 saccades per second, 
and there are also microsaccades.

The general SC architecture has some similarity with the amygdala, and the SC also serves as the adaptive interface to saccades. As such, it mobilizes the frontal eye field (FEF, an attention action area), coordinating with the thalamic mediodorsal and pulvinar nuclei (these project to frontal and object areas respectively) [Krauzlis et al., 2013]. When saccades are executed in adaptive mode, the SC is allowed by the dSTR-SNr path in a way similar to how the BG allows the thalamus [Hikosaka, 2007]. The SC/IC also participate in other aspects of the acute response, including autonomic recruitment [Carr, 2015], DA release [Overton et al., 2014] and possibly innate avoid actions [Shang et al., 2015].

The claustrum. OOA serves to generate maximal stimulus-related BU flow. This flow reaches frontal areas to determine the response to the stimulus. The very first frontal response is to provide feedback TD flow in the spatial direction of the stimulus, in order to identify it and find a response to it (i.e., to start the competition process). This stage of giving attention is mediated by the claustrum, a thin sheet of neurons located between the insula and the striatum. As other areas, most of its neurons are excitatory, with a minority of coordination IINs. It is bidirectionally connected with virtually all abrain areas, the strongest connections being with $\mathrm{mPFC}$ (the cortical area first responding to alerts, which includes the cingulate cortices), MEC (which represents objects in place) and the thalamic nucleus serving frontal cortex, MD.

The claustrum has been argued to segregate sensory flow for attention [Goll et al., 2015]. Supporting a role in focused attention, cortical flow to the claustrum arises from the sensory network, directly (L4) and indirectly via L6. The claustrum-projecting L6 pyramidal neurons are strongly innervated by neurons in all layers, especially L4, and have long slender apical dendrites that reach L1 (unlike other L6 neurons) [Thomson, 2010], like prediction network neurons. Claustrum projections to MEC target the prediction network [Kitanishi and Matsuo, 2017]. Internal connections between excitatory neurons are rare [Kim et al., 2016]. Temporary suppression via electrode stimulation led to immediate loss of volitional (i.e., TD) behavior, unresponsiveness, and amnesia [Koubeissi et al., 2014]. The claustrum promotes $\mathrm{ACh}$, the Rgen mediating the focused attention $\mathrm{N}$ mode: there is altered connectivity of the $\mathrm{ACh} \mathrm{BF}$ with the left insula/claustrum in mild cognitive impairment [Li et al., 2017a], and the claustrum and the supramammillary nucleus activate cortex during rapid eye movement (REM) sleep, the sleep stage at which ACh is most active [Luppi et al., 2017]. Combining these data, the R17 view is that the claustrum transforms sensory alert flow to focused attention. This enhances Goll et al's account by putting it in the context of the $\mathrm{R}$ process.

\subsection{Conditional wait}

Consider the state in which an animal waits immobile for a specific event (condition) to occur, in which case it executes a planned action. We refer to this situation as the conditional. It is common both in nature (e.g., predation) and in experimental paradigms (e.g., perceptual decision making). The R17 account of the wait phase is that both the condition and the planned action are implemented as ordinary $\mathrm{Q}$ process goals. Indeed, mouse motor cortex and thalamus neurons show bidirectional persistent activity during a task delay period, which predicts the specific response [Guo et al., 2017]. When there is large uncertainty about the condition, activation is of the DM network in its general anticipation capacity. As uncertainty diminishes, activation moves to the prediction network.

Total neuronal activity increases during wait, because quax neurons fire at high frequency and continuously innervate additional neurons, including neurons that release Rgens 
(mostly NE and DA, since the conditional is generally an acute state and DA excites the DM and prediction networks via D1R and sustains the goal). This induces a positive feedback loop that mobilizes more and more neurons, resulting in the known experimental phenomenon of climbing (ramped up) activity [Knudsen et al., 2012]. It is not rare to execute the action before the condition occurs ('jumping the gun'), which is due to climbing innervation of the response network by the prediction network ${ }^{45}$, which can cause the action to be triggered by unexpected sensory inputs that are not involved in the condition.

Usage of the decision making network during wait is strongly supported by EEG averaging, which clearly shows negativities that slowly increase in anticipation of an event. Well known cases include the readiness potential, the contingent negative variation, and the stimulus-preceding negativity, in which the event is a voluntary movement, a quick response to an impending stimulus, and an information-carrying stimulus that does not require movement, respectively [Brunia et al., 2012].

\subsection{Working memory}

Working memory (WM) is a well-known wait-like situation. In the basic WM setting, the animal is shown an object (study item) that then disappears, and it needs to execute a specific movement (e.g., click a button with the right hand) when the object (but not others) reappears. During the delay period study items are not physically present yet are kept in some state of activation, and this detachment from physical reality is sometimes viewed as fundamental to higher level cognition. R17 views WM as a variant of the ordinary conditional, the difference being that here none of the condition's features is externally present. Since the condition enjoys no BU support, frontal cortex must continuously generate drive, which may be possible only when it is relatively large (as in primates).

Since the timing of object reappearance is uncertain, delay period activity is in the superficial networks, as evidenced by single unit recording [Arnsten, 2013] and sustained BOLD and EEG negativity [Perez et al., 2012]. Thus, WM can be viewed as part of the DM planning $N$ mode (see below). The sustained anterior negativity increases with the number of auditory items in memory, leveling at 4+ items [Alunni-Menichini et al., 2014]. In addition, Alpha2 NERs, which suppress the execution networks, enhance WM [Wang et al., 2007]. Salient delay period activity has been detected in the inferotemporal cortex, lateral intra-parietal (LIP) cortex, and dorsolateral PFC. Conforming to the content represented by these areas, this activity was sensitive to object identity, object location, and task rules respectively [Perez et al., 2012, Fuster, 2015].

There are well known limits $(7 \pm 2,4)$ on the number of objects that animals can maintain in WM. These stem from feature overlap (interference) within and between objects and responses. Since each object needs to maintain a separate quax mapping it to the correct response, and since there is very high overlap between neurons representing responses (it is the same task after all), having multiple objects increases the probability of quax collapse. This can be alleviated by strategies that strengthen the quacia involved. Humans can additionally use explicit rehearsal or stories in which the objects participate. These are a form of imagery and do not necessarily involve the DM network (see below).

\footnotetext{
${ }^{45}$ Coupled with increased NE and DA that bind to alpha1 NERs and D1Rs, releasing responses suspended by the higher affinity alpha2 NERs and D2Rs (see below).
} 


\subsection{Summary}

In summary of the $\mathrm{Q}$ section, at any given moment, what the brain executes is determined by a matching between sensory inputs and actions. Actions specify (mobilize) their goals and auxiliary actions in decision making mode. Hierarchical quax transitions are triggered by sensory inputs conveying goal attainment. The Q process consists of such a back-andforth interaction between new sensory inputs and responses, and it underlies movements, attention, conditional wait and working memory.

The $\mathrm{Q}$ process is a partial instance of the R process. Non-automated tasks yield alert network flow. Mobilization and alerts determine the population of nodes participating in DM competitions. A focused response is found by BU-driven competition, mediated via GABAergic coordination networks. GABA does not inhibit processing; on the contrary, it is what enables focused, effortful execution.

The Q process explains the important question of how it is that different muscles can learn to operate simultaneously during the same low level operation. For example, when moving the arm to grasp an object, the fingers start moving before reaching the object. This happens because the muscles that move the hand and the fingers are mobilized by higher level action nodes, and nothing prevents them from contracting at the same time because they do not directly compete.

With sufficient practice, the coordinated motion of the different effectors seems to become optimal, since automaticity optimizes energy expenditure. However, contrary to a common view, brain processing does not guarantee optimality of movement, because mobilization patterns acquired early in learning can persist. That is, if you got used to doing something in some way (e.g., due to injury or external constraints), it may persist even if it is very far from a better alternative of performing the action.

\section{The N Process: Functional States}

The $\mathrm{N}$ process is a complete brain instantiation of the $\mathrm{R}$ process (see Table 1 and Figure 2). Each $\mathrm{R}$ mode is promoted by a prototypical $\mathrm{R}$ agent (Rgen). In general, each Rgen promotes its own mode via the flow and coordination networks, excites Rgens promoting cooperating and auxiliary modes, and suppresses Rgens signaling competing modes.

Many Rgens bind to several receptors that couple to different intracellular processes. It is common to have high and low affinity receptors that yield opposite effects. A major reason for this is that low affinity receptors (e.g., D1R (DA), alpha1-NER (NE), alpha7 $\mathrm{nAChR}(\mathrm{ACh})$, which are excited by relatively large amounts of the Rgen, support acute responses, while high affinity receptors support automated responses. Some prominent high affinity receptors, including D2Rs (DA) and alpha2-NER (NE), are suppressive and autoreceptors (i.e., located on the neurons that release the Rgen), and can thereby prevent a positive feedback loop and quench the diffuse (non-synaptic) release of the Rgen, which are not desired in automated responses. Suppressive high affinity receptors are also located on target neurons, and are used to suppress them after their responses, since repeated firing is not needed in automated responses.

Most Rgen receptors are G protein-coupled receptors (GPCRs) that activate Gs, Gi/o or Gq. Gs supports immediate execution by providing energy through adenylyl cyclase, cAMP, and protein kinase A (PKA). Gi/o does the opposite function by inhibiting cAMP. Gq supports continued execution via protein kinase $\mathrm{C}$ (PKC), whose major forms increase intracellular calcium to excite the cell.

There are five major $\mathrm{N}$ mode switch hubs in the brain: the amygdala, the hypothalamus 
(HT), which controls the release of most Rgens, mPFC, which is the first cortical area that responds to alerts and which innervates Rgen nuclei, the BG, which switch between innate, acute and automated responses, and the habenula $(\mathbf{H b})$, which switches between focused execution (DA, ACh) and DM (SER).

\subsection{The hypothalamus: $N$ mode hub}

The hypothalamus (HT) is comprised of a set of nuclei, each sensitive to and releasing a unique set of Rgens. HT neurons receive inputs conveying $\mathrm{N}$ mode information from the circulation, the brain (the extended amygdala, mPFC), and the cerebrospinal fluid (CSF), and project back to communicate $\mathrm{N}$ mode switches.

The main HT nuclei and Rgens are as follows. The arcuate (ARC) nucleus is a major input nucleus for energy (hunger and satiety) and sex signals. It has three main neuronal populations, expressing AgRP/NPY, proopiomelanocortin (POMC), the precursor to BEND and the melanocortins alpha-MSH and ACTH, and kisspeptin (KISS1). The paraventricular (PVH) nucleus primarily releases AVP, CRH, OT and POMC, to control alertsuccess switches. The tuberomammillary (TMN) nucleus contains histamine neurons. The lateral HT (LH) primarily releases OX and MCH to mediate the switches between internal needs and their satisfaction and between wakefulness and sleep. The dorsomedial (DMH) nucleus is involved with thermoregulation and circadian rhythms, including wake and sleep (via OX). The ventromedial (VMH) nucleus is involved in mating and aggression, via sex hormones, SP and NOC. The preoptic and supraoptic areas (especially the medial preoptic area (MPOA)) release OT, AVP, KISS1, gonadotropin-releasing hormone $(\mathrm{GnRH})$ and NOC, and are involved in mating and aggression as well.

The role of the HT is to control the initiation and termination of the $N$ process, by releasing need promotion agents (OX, AVP, SP, HIST, kisspeptin) and all termination agents that are not strictly local (MCH, BEND, OT, CRH, GCs (indirectly). DYN, ENK, and ECBs are local). Food and sex agents act on the HT to work via other agents, but also act on the brain directly. The non-local Rgens not released by the HT are those internally used by the brain for its operation (SER, NE, DA, ACh) ${ }^{46}$.

\subsection{Internal need: orexin (OX), arginine vasopressin (AVP), sex hor- mones}

Internal needs are signaled by the body and excite brain paths involved in their satisfaction. Every internal need pathway includes agents that directly convey the need from the body, neurons directly sensing these agents, downstream targets of these neurons, and learned representations, including innate+ ones. In the same vein, the satisfaction of an internal need involves signals indicating imminent satisfaction, neurons that sense these signals, movement-triggering agents excited by the above or in DM mode when planning responses, and internal satisfaction agents excited by the above. Need-sensitive neurons are located all over the brain, and are especially dense in valence areas. For example, the BNST and hippocampus express sex hormone receptors. In addition to these $\mathrm{N}$ process aspects of internal needs, each need has innate responses activated by hard-wired sensory stimuli and generalized in the innate+ system.

The three main internal needs are food (energy), water and minerals, and mating. Metabolism and reproduction are managed by complex systems whose detailed descrip-

\footnotetext{
${ }^{46}$ SER and NE are also intensely used outside of the brain.
} 
tion is beyond the scope of this paper; here we briefly mention only the main brain agents. Important food agents such as insulin, leptin, adiponectin, glucagon-like peptide 1 (GLP1), cholecystokinin (CCK), and PYY, and sex agents such as progesterone, DHT and DHEAS are discussed elsewhere.

The need for food is conveyed by ghrelin (signaling an empty stomach) and by reduced glucose levels [Morton et al., 2014]. These signals are sensed by agouti-related peptide (AgRP) / neuropeptide Y (NPY) neurons in the HT ARC, and by LH OX neurons. Satisfaction is indicated by increased glucose and fat levels, ARC POMC neurons, BEND and OT (see below). The need for water and salt is conveyed by angiotensin II, vasopressin, and osmolarity state, and sensed by neurons in the organum vasculosum of the lamina terminalis (OVLT), median preoptic area (MnPO) and subfornical organ (SFO) [Zimmerman et al., 2017]. These excite PVH AVP neurons. The relevant innate actions (e.g., licking and chewing) are mostly triggered by mouth sensors (e.g., ones sensitive to glucose and fats).

Mating and reproduction depend upon steroid sex hormones (androgens such as testosterone, estrogens such as the human estradiol), whose pulsatile release from the gonads (testes, ovaries) is promoted by KISS1 neurons via GnRH neurons and pituitary luteinizing hormone (LH) [Clarkson et al., 2017a $]^{47}$. The need for mating is different from hunger and thirst in that it is conveyed via pulsatile (acute) release rather than via gradually increased excitation, and in being controlled by a negative feedback mechanism. In both aspects, as well as in the role of the pituitary, sex hormones behave like glucocorticoids, which are also steroids (see the energy $\mathrm{N}$ mode below). Mating innates (the female lordosis posture and the male sexual responses) are mostly triggered by pheromones and supported by the sex hormones.

The need system is interconnected such that neurons promoting needs excite each other and inhibit satisfaction neurons, and vice versa. For example, OX, AVP and AgRP neurons are bidirectionally connected, and testosterone excites AgRP neurons. AVP is an important mediator of alerts, which use its effect on the blood for recruiting energy. This need group is opposed by the satisfaction group, whose main Rgens are BEND (POMC) and OT.

Orexin. The main agent that conveys need to the brain is orexin (OX) (also called hypocretin), via VTA (DA) projections. OX [Chen et al., 2015] is released by neurons in the lateral (LH), dorsomedial (DMH) and perifornical hypothalamus. It has two isoforms, OX-A and OX-B, generated from the same precursor protein. OX-A equally binds to OX1 and OX2, and OX-B has a much higher affinity for OX2. Both receptors are GPCRs. OX1 is excitatory via $\mathrm{Gq}$, and $\mathrm{OX} 2$ signals via $\mathrm{Gq}$ and $\mathrm{Gi} / \mathrm{o}$. OX neurons are highly excitable, having much stronger glut inputs than GABAergic ones and expressing calcium permeable AMPARs [Gao and Hermes, 2015]. They sense glucose and many are directly excited by low glucose and inhibited by rising glucose. They receive inputs from need and valence areas such as the amygdala, BNST, HT nuclei and the lateral septum, and widely affect the brain, including Rgen nuclei, the PAG, the parabrachial nucleus, NTS, PVN, and the medulla.

OX promotes and is promoted by the urgent acute response via NE, CRH, DA and ACh. In particular, OX incites to action via dense projections to the VTA, where DA neurons expressing OX1 are excited by OX. At high doses, OX-B opposes the non-urgent DM response by decreasing glut inputs onto DRN SER neurons via presynaptic ECBs [Haj-Dahmane and Shen, 2005]. At lower doses, OX excites SER neurons to promote non-urgent DM [Sinton, 2008]. OX neurons receive inputs from the NAc, which is the target of VTA DA. OX excites thalamic nuclei [Govindaiah and Cox, 2006] and need areas including mPFC, mostly via OX1. OX1 is essential for some of the effects of morphine (a

\footnotetext{
${ }^{47}$ See the energy mode below for their release by the adrenal cortex.
} 
mu-opioid receptor (MOR) agonist, see BEND below) on VTA DA neurons [Baimel and Borgland, 2015].

Behaviorally, OX promotes both innate internal need states and adaptive actions to address all kinds of needs [Sakurai, 2014]. Since the need system is tightly interconnected, OX neurons participate in external needs as well, e.g., rapidly increasing activity in response to air-puff and immobilization stress [González et al., 2016a].

Consistent with its internal need signaling role, OX is associated with the classical brain arousal system and promotes wakefulness, mostly via OX2. Lack of OX neurons causes narcolepsy. The internal need most strongly related to OX is that for food. OX neuron activity rapidly decreases milliseconds after eating onset, due to their sensitivity to the glucose molecule but also by calorie-less food [González et al., 2016b], showing that $\mathrm{OX}$ is affected even by eating actions alone. OX neurons are directly excited by ghrelin and inhibited by leptin, and deletion of OX neurons produces obesity. Feeding behavior induced by MOR agonists in the NAcSh (see below) depends on OX1R activation [Zheng et al., 2007]. The other prominent Rgen released by lateral HT neurons is MCH, also associated with feeding (below). Via its VTA projections, OX also has a role in sex-driven conditioning [Di Sebastiano and Coolen, 2012].

AVP. AVP [Stoop, 2012] is released by HT PVH, preoptic, supraoptic (SON) and DMH neurons, and by the BNST, CeM, olfactory bulb and the LC. AVP is excitatory, working through the Gq-coupled V1a and V1b receptors and the Gs-coupled V2R (and also via the OTR). In addition to synaptic release, AVP undergoes dendritic release, which facilitates wide and prolonged distribution via the cerebrospinal fluid [Sewards and Sewards, 2003]. AVP receptors are mostly present in valence areas, including the amygdala CeM and mPFC.

AVP is associated with three types of needs, water, mating and aggression. Vasopressin has a peripheral role as an anti-diuretic, increasing urine concentration. AVP neurons signal the need for smooth blood flow and water (i.e., thirst). AVP promotes olfaction-triggered innate sex and aggression (including non-conspecific aggression) responses, and is increased by testosterone to promote both response types [Vyas, 2015]. AVP neurons are active in situations indicating the need for mating, and are involved in aggression [Sewards and Sewards, 2003]. Like CRH, AVP stimulates the release of ACTH (see the extended alert and protection modes below), supporting its role as an acute need agent. VP is closely structurally related to oxytocin (OT), which promotes fluid-related satisfaction and conspecific interactions (below).

A unified view is that both AVP and OT promote urgent approach innates, AVP before and OT during need satisfaction. The needs promoted by AVP are related to each other in that thirst and mating both involve fluids and effort, and aggression is tightly related to mating (see SP below). In all cases, AVP signals the need for making an energy-consuming physical effort to address external challenges.

\subsection{Alert: norepinephrine (NE)}

The alert mode is generally triggered by strong surprise, which has both single neuron and system effects. At the single neuron level, expectation means that the cell is excited to some extent. Conversely, surprise means that the cell is hyperpolarized for some time. In many neuron types, when a hyperpolarized neuron suddenly receives enough inputs to get activated, its output is generally amplified to be stronger than that of activated partially excited neurons, showing a high frequency burst of action potentials. For example, after having been hyperpolarized for more than $100 \mathrm{~ms}$, thalamic neurons enter burst mode due to T-type $\mathrm{Ca} 2+$ channels causing a low-threshold spike, on top of which can ride a series of 
high frequency APs [Sherman and Guillery, 2013, Cheong and Shin, 2013].

At the system level, expectation is manifested by the molding of neuronal terrain to channel incoming flow to a response. This is done via excitation of the channeling path, possibly coupled with inhibition along the path's boundary. Surprise-induced flow is not restricted in this way, and can rapidly propagate in the alert and DM networks in neighboring and far-away nodes. The strong burst firing of sensory and alert neurons induced by the surprise may be strong enough to activate their node's execution neurons. Thus, surprises can generate strong high frequency activity in all networks, including the execution networks. Moreover, since response neurons throughout cortex project to DA nuclei, surprises yield a burst of DA release (see below).

The role of valence. Small surprises occur continuously during wakefulness and usually do not trigger an alert mode (see OOA above). There are two typical cases in which an alert mode occurs: execution of a valenced or OOA innate (e.g., pain, quick external movement, food $)^{48}$, or the unexpected occurrence of an event (usually appearance of an object) associated with such an innate. Note that these include positive innates, e.g., when seeing a sexually attractive person or even a friend (see skin response below). The amygdala is a key mediator of alert urgency in both cases, through CeA (in both cases) and BLA (in the latter case). As noted above, CeA projects to Rgen nuclei and the HT, BLA projects very strongly onto cortex, and the thalamus conveys direct sensory inputs to $\mathrm{mPFC}^{49}$. Thus, valence areas receive strong alert flow.

The alert mode is assisted by NE, AVP, CRH, SP and histamine. It has both brain and autonomic effects, the latter being quite similar to the initiation of classic stress response.

Norepinephrine (NE). NE (also called noradrenaline) signals alert and has different roles in the body and the brain. In the body, it induces the mobilization of energy resources through the sympathetic branch of the autonomous nervous system (S-ANS). The S-ANS is stimulated by HT neurons (alerted by $\mathrm{CeA}$ ) yielding NE secretion from the adrenal medulla. The resulting effects include increased heart rate, blood pressure and blood flow to major organs (e.g., the brain, heart, muscles), pupil dilation, accelerated respiration, etc.

In the brain, NE neurons are primarily located in the locus coeruleus (LC), a small bilateral brainstem nucleus, and in a few additional brainstem nuclei (e.g., in the NTS). The LC is innervated by a wide range of regions including cortical valence areas, the CER, and innate-related nuclei (including the amygdala CeA, the HT, and the SC), and projects extensively to virtually all brain areas (except the BG, where DA supports alerts). It has particularly strong projections to $\mathrm{mPFC}$ and to areas involved in spatial attention such as the parietal cortex.

NE is commonly released after the real occurrence of a surprise, but can also result from prediction of alert states (e.g., when you get into a situation known to be dangerous). Valence areas are key mediators in both cases.

NE is excitatory via alpha1 and beta1-3 NE receptors (NERs, also denoted adrenoreceptors (ARs)), and suppressive via alpha2 NERs (Gi/o). Here we present a novel assignment of roles to these receptors.

The affinity of beta NERs to NE is higher than that of alpha1 NERs, and they couple to Gs, which supports immediate execution, while alpha1 NERs couple to Gq, which

\footnotetext{
${ }^{48}$ Note that innate-triggering sensory input would trigger innate execution regardless of whether it is expected or surprising. However, expected innate execution would not yield the alert $\mathrm{N}$ mode, which is an adaptive response.

${ }^{49}$ The PFC area that receives this flow is a vmPFC area called infralimbic (IL) mPFC in rodents, roughly homologous to human subgenual cingulate cortex [Morris et al., 2016].
} 
supports extended execution. Beta NERs are mostly expressed in the sensory and alert networks, while alpha1 NERs are mostly expressed in the DM network and in HT PVH CRH neurons [Goldman-Rakic et al., 1990]. Thus, beta NERs support immediate responses, including innate (e.g., cardiac) ones, while alpha1 NERs support adaptive decision making. Alpha1 NERs are also essential for the transient excitatory effect of GABA during alerts (see above).

The affinity of alpha2 NER to NE is greater than that of alpha1 and beta NERs [Arnsten and Pliszka, 2011], which points to a role in automated responses (suppressing firing neurons after a transition) and low alert situations (e.g., extended alert, below). According with this role, alpha2 NERs act as autoreceptors to suppress ongoing LC NE release. Alpha2 NERs are colocated with HCN channels and close them [Wang et al., 2007]. HCN channels are expressed only in response network pyramids [Sheets et al., 2011] and support repeated firing at slow rates (i.e., the automated mode). Alpha2 NERs may also act in the initial stages of alerts to pause the ongoing task to promote attention to the alert. NE opposes the ongoing task via its mutual suppression of DA and SER [Guiard et al., 2008, Tassin, 2008].

In summary, innate+ activation yields NE, which recruits energy, pauses ongoing activity, enhances sensory inputs, and widely excites the predecision networks to induce an acute response.

\subsection{Extended alert: corticotropin-releasing hormone (CRH)}

Learned responses are resolved and executed immediately. Acute responses result from unexpected events, and involve the activity of a large number of neurons for an unknown duration. The $\mathrm{N}$ process has an extended alert mode that has two actions, recruiting longer term energy resources and sustaining the alert mode. The latter is needed because the alert mode itself is triggered by specific events, not by general alert situations such as places associated with past alerts and threats that are still far away.

Extended alert is mediated by corticotropin-releasing hormone (CRH) (also factor, CRF). CRH is released by hypothalamic PVH, amygdala, and BNST neurons. CRH binds to $\mathrm{CRH} 1$ and $\mathrm{CRH} 2$ receptors, which are excitatory via Gs. The $\mathrm{CRH}$ family includes also the urocortins and their receptors. PVH CRH neurons are directly excited by brainstem NE neurons [Uchoa et al., 2014], and release CRH into the circulation. CRH may also be distributed through the CSF like AVP [Sewards and Sewards, 2003]. CRH action in the pituitary gland promotes ACTH, which yields adrenal release of energy management Rgens. CRH also has peripheral roles in birth.

In the brain, Rgen nuclei, the amygdala and the hippocampus express CRH1R, and to a lesser extent CRH2R. In the amygdala, CRH is mostly released by the CeL part of CeA [Day et al., 1999]. There is substantial co-expression of CRH and DYN in CeA and of CRH and DOR (the ENK receptor) in BLA and CeA [Reyes et al., 2017]. CeA neurons coreleasing CRH and DYN project to the LC, promoting NE release [Reyes et al., 2011]. $\mathrm{CRH}$ reduces amygdala AEA tone (see the protection mode), increasing the excitability of BLA projection neurons and yielding anxiety-like behavior [Gray et al., 2015]. CRH promotes HT OX [Winsky-Sommerer et al., 2004] but suppresses AgRP neurons [Sominsky and Spencer, 2014], showing that while OX signals general needs, the AgRPs signal internal needs.

These data imply that $\mathrm{CRH}$ has a dual role. First, it recruits energy via the pituitary actions of HT CRH neurons. This role is discussed in the energy mode below. Second, it sustains alerts beyond their initial triggering. It is released in alert situations, especially those related to places and longer term actions, as indicated by BNST release. CRH ex- 
cites NE, but unlike acute NE it does not promote rapid responses but an extended state of low alert associated with reduced action. Indeed, CRH and SER mutually promote each other [Forster et al., 2008, Marcinkiewcz et al., 2016], and both promote DM and oppose immediate action. The co-expressed DYN and ENK are released after prolonged neural activity, supporting an extended acute mode. The IINs of the cortical CCN and ECN express CRH binding protein, which inhibits CRHR1 [Ketchesin et al., 2017]. These networks are involved at the final stages of the alert mode, and clear CRH once alert is replaced by execution.

Slower CRH release can yield reduced excitation of the LC, resulting in low NE release that activates alpha2-NERs and suppresses adaptive responses. This promotes innate responses, especially avoid ones (according with DYN corelease). Slow responses coupled with a steady low alert are the prototypical behavior when getting into dangerous situations.

CRH2R may support automated responses through its primary ligand, urocortin 3. $\mathrm{CRH} 2$-expressing pBNST neurons are anxiolytic and reduce GC release [Henckens et al., 2017].

$\mathrm{CRH}$ is a major factor in sustained (chronic) anxiety. Suppression of cortical responses explains why low intensity stress impairs cognitive function (thinking, WM, memory access) and is associated with excessive hesitation. CRH also has a major role in addiction, discussed elsewhere.

\subsection{Decision making, planning: serotonin (SER)}

Alerts induce the activation of several adaptive response alternatives, which compete in the DM mode to win the response decision. There are two different DM modes, urgent (competition) and non-urgent (planning), the former using Rgens that shorten it and push towards a decision (DA and ACh, see below), the latter using an Rgen that prolongs it, serotonin (SER) $)^{50}$. Response urgency is conveyed by innate+ valence flow and by the release capacity and receptor expression of the three Rgens. Here we present a detailed novel account of the role of SER and its receptors.

SER is an ancient molecule promoting photosynthesis in plants and having various related peripheral roles [Azmitia, 2010]. The essential amino acid tryptophan is a SER precursor (also of melatonin and the kynurenines). SER acts through at least 14 types of metabotropic G protein-coupled receptors, the main ones being SER1a, SER1b and SER5, which are suppressive via Gi/o, SER4, SER6 and SER7, which are excitatory via Gs, and SER2a, SER2b and SER2c, which are excitatory via Gq. There is one ionotropic SER receptor, SER3, which is excitatory and close to nicotinic ACh and GABAA receptors in structure. All SER receptors promote neurite growth.

SER is released by neurons in the dorsal and median raphe nuclei (DRN, MRN). The DRN is connected with a wide variety of brain areas, including PFC (specifically mPFC [Maier, 2015]), secondary motor areas, the lateral habenula (LHb), the hypothalamus, DA and other Rgen nuclei, and the BG [Dorocic et al., 2014]. The MRN is connected most strongly with the medial habenula, IPN and the hippocampus, all of them areas in which ACh acts strongly (below). Thus, the DRN and MRN are geared towards the DA and ACh systems, respectively. According with the general connectivity pattern, adaptive areas (PFC, LHb) project to the DRN bilaterally, while innate areas (LH, POA, SNc, amygdala) project ipsilaterally [Zhou et al., 2017].

\footnotetext{
${ }^{50}$ The formal term is 5-hydroxytryptamine $(5-\mathrm{HT})$. We use SER for readability, including in receptor names, e.g., SER1a instead of 5-HT $1 A$.
} 
Behaviorally, SER is associated with patient waiting for reward [Fonseca et al., 2015], which is a decision making situation. DRN SER levels are higher during waiting for a delayed reward, and SER neurons stop firing only when rats give up [Miyazaki et al., 2011]. Tryptophan depletion promotes waiting impulsivity in humans [Worbe et al., 2014]. Similarly, SER reduces panic and avoid innates in general [Graeff, 2017], and low SER levels are associated with high trait aggression in males [da Cunha-Bang et al., 2016]. SER is not limited to positive situations, as SER and CRH (extended alert) mutually excite each other, according with reduced action during general need-driven DM.

Mechanisms. SER promotes the DM mode in four ways. First, it suppresses fight and flight (panic) innate responses in the amygdala CeA via SER1a [Li et al., 2012]. Second, it suppresses the alert mode by opposing NE and inhibiting CeA-HT projections. Third, it suppresses responses. SER inhibits $\mathrm{mPFC}$ pons-projecting response network neurons via postsynaptic SER1a [Avesar and Gulledge, 2012]. SER1aRs are mostly located on the axon initial segment, exerting rapid action [Czyrak et al., 2003]. High doses of SER, as would be released during acute situations, suppress motoneurons via SER1a on the axon initial segment [Perrier and Cotel, 2015]. Thus, SER cooperates with the response suppression network. SER also suppresses contralateral and ventral hippocampal inputs onto the mPFC execution networks via presynaptic SER1bRs, while not harming BU thalamocortical flow [Kjaerby et al., 2016]. Similarly, SER2a excites cell body-targetting IINs, including those in the response suppression network [Jakab and Goldman-Rakic, 2000]. SER suppresses Rgens promoting execution, including ACh [Fink and Göthert, 2007], DA (see below), and MCH [Devera et al., 2015]. SER suppresses quick cerebellum responses by inhibiting parallel fiber-PC synapses [Kawashima, 2017]. SER nuclei are not directly innervated by the BG, showing that SER is not needed for response execution. It exerts an overall inhibitory effect on automated BG execution by suppressing STN inputs and outputs via SER1b [Ding and Zhou, 2014].

Finally, SER directly promotes planning. It excites the DM cortical network via SER2a [Avesar and Gulledge, 2012], mostly on apical dendrites [Jakab and Goldman-Rakic, 2000], excites the competition coordination network (CCN) neurons via SER3, and excites the amygdala BLA, which drives the DM network, via SER2c [Li et al., 2012]. These actions have the combined effect of prolonging the competition process and broadening the range of solutions considered during decision making. In addition, SER and CRH are mutually supportive (SER via SER2c), to promote slow, vigilant responses during decision making. As discussed extensively under imagery below, planning involves response network neurons in high level frontal areas. SER2c excites a subset of mPFC L5 pyramidal cells via SER2c [Santana and Artigas, 2017]. Planning also primes motor circuits, and indeed, SER facilitates mossy fiber-deep nuclei synapses [Kawashima, 2017].

The relationship between SER and DA seems paradoxical. On one hand, they should oppose each other, since SER prolonges DM and DA shortens it (see also the habenula below). Indeed, SER reduces DA [Guiard et al., 2008, De Deurwaerdère and Di Giovanni, 2017] (specifically SER2c [Bailey et al., 2018]). On the other hand, DA excites SER [Guiard et al., 2008], the SER reuptake inhibitor fluoxetine greatly increases PFC DA [Klomp et al., 2014], and DRN stimulation strongly reinforces behavior and releases SER (and glut) in rewards [Qi et al., 2014]. This can be explained as follows. DA excites SER via D2R using non-selective cation channels [Haj-Dahmane, 2001]. That is, this occurs in the automated mode, in which there are no urgent DM responses. Fluoxetine increases DA by stimulating SER1a/1b autoreceptors, which downregulates SER and reduces its suppression of DA. SER should be rewarding, because it is part of the acute response. In addition, there are glut DRN projections onto the VTA, whose activation reinforces behavior [Liu 
et al., 2014, McDevitt et al., 2014].

Like other Rgens (NE, DA, see below), small amounts of SER support automaticity, e.g. by SER1a and SER1b autoreceptors and by facilitating motoneurons via dendritic SER2 and SER7 [Kawashima, 2017, Perrier and Cotel, 2015]. In addition, SER7 cooperates with HCN (Ih current) channels [Santello and Nevian, 2015], which induce a membrane potential that is close to the firing threshold, thereby supporting relaxed oscillations. In cortex, HCN channels are present on response network neurons. SER4 binds to SER with high affinity and couples to Gs, which induces immediate responses. Hence, SER4 seems to support automated responses. Indeed, it is widely expressed at the hippocampus and the BG, and is known to facilitate cognition [Hagena and Manahan-Vaughan, 2017]. SER2c excites dSTR cholinergic IINs (CINs, see below), which support motor areas, via SER6 and SER7 [Virk et al., 2016].

In summary, SER promotes decision making, flexibility and creativity, favoring prolonged planning and thinking (patience) over acting. It reduces alerts, response urgency and innate-driven (emotional) responses without impairing long term motivation.

\subsection{Competition, prediction, goal-setting: dopamine (DA)}

The urgent DM response to alerts involves the excitation via disinhibition of response candidates to quickly induce competition. After competition resolution, the execution of the resulting focused response is sustained as needed. Competition and the subsequent sustained execution are supported by dopamine (DA) and acetylcholine (ACh). During execution, DA defines and sustains goals and prepared actions by exciting the prediction network, while ACh supports the response network. DA has a greater effect on the N process (both long- and short-term), and thus its role can be said to support goal-setting and decisions. DA affects frontal and motor areas, while ACh has a greater effect on short term $\mathrm{Q}$ process execution in sensory areas. Here we present a detailed novel account of the role of DA and its receptors.

Most abrain DA is released by neurons in two midbrain nuclei, the ventral tegmental area (VTA) and the substantia nigra pars compacta (SNc). The VTA and SNc receive inputs from virtually all brain areas. Markedly strong inputs arrive from the BG, where most MSNs project to GABAergic neurons in the VTA/SNc (some project directly to DA neurons, see striosomes below). Cortico-VTA projections arise from the response network. DA neurons project extensively throughout the brain, especially to the BG and frontal cortical areas. The VTA projects to motivation and task areas, while the SNc focuses on motor and sensory areas. The bidirectional connections of DA nuclei with the STR form distinct circuits [Lerner et al., 2015], and specific VTA/SNc subsets project to specific cortical areas [Yetnikoff et al., 2014]. Hence, R17 posits that the cortico-BG-thalamocortical loops include their own DA supply. That is, when a cortical node is active in response mode, it provides itself and its $B G$ path with DA.

Receptors. DA acts through two families of G-protein coupled receptors. D1Rs (D1, D5) are excitatory via Gs and Gq, and their effect prolongs for a long time after DA clearance [Seamans and Yang, 2004]. D2Rs (D2-4) have a higher affinity than D1 and thus a faster effect. D2Rs are mainly inhibitory via Gi/o, but cortical D2Rs have been shown to induce a Gs-like afterdepolarization involving repeated low amplitude spiking for hundreds of milliseconds [Robinson and Sohal, 2017]. D1Rs are abundant in the internal networks, especially in the DM network [Arnsten, 2013, Clarkson et al., 2017b] and in the BG direct path, while D2 favors the response network [Gee et al., 2012] and the BG indirect path.

Behaviorally, D1 is associated with subjective reward value [Schultz, 2016], risk taking 
[Rutledge et al., 2015], motivation, effort, perseverance, and vigor [Salamone and Correa, 2012]. Seemingly paradoxically, DA is also released in aversive events [Bromberg-Martin et al., 2010], including phasic DA release during aggression [Anstrom et al., 2009]. In R17, the role of $D 1$ is to promote and sustain $N$ process decisions via competition and prediction, with an emphasis on urgent decisions, explaining all of these effects. The goal of the $\mathrm{N}$ process is to satisfy needs, explaining DA's association with rewards and motivation, but also its involvement with aversive events, which indicate external needs. DA opposes prolonged DM, explaining risk taking. DA sustains decisions, explaining perseverance and effort. DA is released during charging actions preceding $\mathrm{N}$ process termination by consume and fight innates (see BEND below), explaining vigor. Indeed, inhibiting VTA DA neurons reduces the initiation and maintenance of motivated responses [FischbachWeiss et al., 2017]. DA's excitation of the prediction network explains why DA promotes movement, since the activation of goals promotes actions to satisfy them.

Competition. DA and ACh cooperate, but for readability we describe their actions separately. DA supports competition as follows. When surprises yield high frequency bursts of response neurons, the effect on the DA neurons that they innervate is the well known phasic DA response to surprise ${ }^{51}$. Phasic DA promotes competition in both the BG and in cortex. In the BG, which enjoy massive DA innervation, the quickly rising DA levels first suppress the indirect path via D2Rs and then strongly excite the direct path via the lower affinity D1Rs. The effect of D2Rs is to suppress executing automated actions and any inhibition that these may exert on novel competition. The effect of D1Rs is to widely open the direct path. Since basically all cortical nodes project to the direct path (both their prediction and response neurons), this allows all competing nodes access to the thalamus, which is essential for the execution of adaptive responses. Amygdalar projections to the STR enjoy a similar advantage. The effect of DA in cortex is similar. It suppresses response neurons via D2 and excites the internal (DM and prediction) networks and higher level response neurons via D1. DA also opposes competing $\mathrm{N}$ modes, e.g., via the LC and the amygdala [Kim et al., 2017b]. Thus, cortical DA excites competition participants, and BG DA allows them access to the thalamus.

DA supports competition, but it does not support prolonged competitions. DA reuptake in the BG is very fast, so only strongly pulsing nodes retain their D1 BG path. This means that once a competing node starts losing, its BG path closes, accelerating its loser status. In other words, competition is promoted by DA, is resolved via coordination networks, and its result is consolidated by DA (and ACh, below). DA innervation of cortex is diffuse and its reuptake is slow, which means that competition winners continue providing their DA supply and enjoy sustained support via D1Rs, while losers are suppressed via D2Rs (they are located at a distance and receive smaller DA amounts). The slow cortical reuptake of DA explains part of the difference between DA and ACh. Both topographically innervate cortical nodes, but ACh reuptake is very fast. Thus, DA is capable of sustaining goals, while ACh is more suitable for promoting responses.

Note the difference between the alert and the DA competition/decision responses with respect to surprise. The alert response is mainly activated by the amygdala and targets the whole brain, while the DA response is activated by cortical nodes and targets limited cortical areas. In other words, alerts are very quick, take into account only the valence of the input, and do not suggest an actual response, while competitions occur somewhat later, take into account the input's specific content, and yield responses.

\footnotetext{
${ }^{51}$ There are actually two phasic DA responses induced by different alerts, the first by OOA and the second by stimulus identity [Redgrave and Gurney, 2006, Schultz, 2016].
} 
Transitions in automated responses. DA supports flexible responses, but it also has an important role in automated ones. When a response neuron fires during automated execution, it drives its DA projections, which may promote the relaxed form of DA neuron activity known as tonic DA release (in addition to inherent activity). The small amount of released DA excites D2R autoreceptors on DA neurons to quench their firing and prevent the release of large DA amounts (which would trigger an acute response). The small amount of DA reaching the response path in cortex and the BG streamlines automated transitions via D2R-mediated suppression or sustained oscillation of the response and execution coordination networks after neuronal firing (see action termination above). Thus, the role of D2Rs is to support automated action transitions, via a brief suppression and subsequent excitation of the cell [Robinson and Sohal, 2017]. This explains the role of the indirect path in habit acquisition (below), and why D2Rs excite the execution coordination network only after automaticity [Tseng and O'donnell, 2007].

Recently, a PFC prediction network population expressing D3Rs but not D1 or D2 has been identified, where D3R targets calcium channels on the axon initial segment to suppress burst firing [Clarkson et al., 2017b]. D3 belongs to the D2 receptor family, and may facilitate prediction network transitions (possibly transitions involving 'ignore' actions, complementing the response suppression network).

The direct path trains the indirect path. After competition results are consolidated in the direct path, the indirect path also starts being active. Indirect path MSNs receive excitatory inputs from the winners, since the cortical execution networks (and possibly the amygdala) innervate the indirect path too. Moreover, direct and indirect MSNs are mutually connected, so active direct path MSNs can send coordination requests to indirect MSNs. At this point both paths are active and convey focused response flow, allowing the thalamus to sustain the executing focused quax. In this way, the direct path bootstraps the indirect path, training it to automate the executing responses.

Cortical learning results in reduced bursts and thus reduced phasic DA events. Hence, the direct path is gradually less active as automaticity proceeds. Indeed, direct neurons get excited and activate before indirect ones when starting an attended action [Sippy et al., 2015], and in a motor training paradigm there was strong dorsomedial STR D1 reduction after the first execution, while dorsolateral STR D2 shows progressive reduction after extended training [Sommer et al., 2014].

Note that in the absence of another alert, new cortical nodes joining the Q process during execution activate only the indirect path, since the direct path is blocked by the lack of DA. Indeed, the direct path is essential for the initiation of attended actions, while execution of learned sequences mostly uses the indirect path [Agnoli et al., 2013]. Cortical sensory and internal need areas, which are more involved in action initiation, preferably target the direct path, while motor areas target the indirect path [Wall et al., 2013, Reiner et al., 2010]. The direct path is known to support vigorous movement, and both paths are used during everyday actions [Cui et al., 2013].

Note that the R17 account of the BG is dramatically different from the accepted view, which posits that the direct path supports movement while the indirect path stops movement, and from another popular view in which the indirect path selects between competing actions [Graybiel and Mink, 2009]. In particular, the fact that the indirect path contains two consecutive GABAergic links before GPi does not imply that it cannot support movement. Rather, it supports movement via coordination instead of via disinhibition. Probably for this reason, it allows lower frequency (i.e., automated) oscillations than the direct path, and it needs sustained excitation from the STN. Indeed, focused GPi excitation in low DA states can induce excitatory thalamic motor signals [Kim et al., 2017a], and the acquisition 
of habits yields plasticity in the indirect path [Shan et al., 2015]. Additional strong support for the R17 account is provided by CINs, see ACh below.

In summary, D1 promotes acute (flexible, non-automated) responses, pushing towards making a TD response decision and executing it. It promotes response content via a general excitation of the DM and prediction networks, including the direct BG path, and promotes execution of the emerging focused response through sustained excitation of these networks. Flexible responses are first formed in the direct BG path, which trains the indirect path. D2Rs, whose activation is triggered by responses, promote automated transitions and quench DA as autoreceptors. Long-term $\mathrm{N}$ process decisions are induced by needs and sustain need-driven goals, so the long-term role of DA can be termed motivation. For DA's role in promoting short need-driven charging decisions see also BEND below.

\subsection{Response execution, attention: acetylcholine (ACh)}

Acetylcholine (ACh) supports competition and focused response execution in three main ways. First, like DA, it supports disinhibition during competition, consolidation of competition results, and sustained focused execution. Due to its specific effect on action-object connections, ACh can be viewed as promoting attention. A wealth of evidence supports a role for fast ACh release in promoting increased attention to stimuli and in subsequent plasticity [Sarter et al., 2016]. In addition, ACh excites the response network and suppresses the internal networks [Baker et al., 2018]. Second, ACh is the agent used in motoneuronmuscle connections, i.e., the means by which neurons induce strong muscle contractions. Third, ACh is used to end the alert mode through the parasympathetic ANS (mostly through the vagus nerve), yielding pupil constriction, slowing down of heart rate, reduced blood pressure, etc. Thus, ACh emphasizes the short term sensoriobject aspects of focused execution.

In the brain, $\mathrm{ACh}$ is released by nuclei groups in the basal forebrain (BF, midbrain) and the brainstem [Mesulam, 2013]. The BF group includes the nucleus basalis of Meynert, the medial septum, and the diagonal band of Broca, and mediates ACh's role in focused execution. These nuclei provide massive topographic ACh innervation to cortex, the hippocampus, and the amygdala. ACh mostly targets the superficial cortical layers. A GABAergic BF population also strongly innervates the amygdala [McDonald et al., 2011]. ACh release into the abrain is mostly driven by widespread cortical, amygdalar and HT projections to the BF. The major cortical inputs originate from areas involved in attention, mostly mPFC [Danielmeier et al., 2015].

The brainstem ACh group includes the pedunculopontine (PPN) and laterodorsal pontine tegmental (LDT) nuclei, which promote the ACh N mode. The LDT is bidirectionally connected with need-related areas (medial thalamus, vSTR, VTA, DRN, HT, amygdala, pontine reticular formation), while the PPN supports motor execution. It is innervated by $\mathrm{M} 1$, is bidirectionally connected with the BG (dSTR), and provides ACh to the thalamic $\mathrm{CM} / \mathrm{Pf}$ and SNc [Mena-Segovia, 2016].

Receptors. ACh acts through two families of receptors, nicotinic (nAChR) and muscarinic (mAChR). nAChRs are ionotropic and excitatory, allowing an influx of positive ions. They desensitize very quickly. There are several nicotinic receptor forms, the two most common ones being alpha7 and alpha4beta2. Alpha7 nAChRs are calciumpermeable, allowing strong excitation, and have a low agonist affinity. Alpha4beta2 nAChRs have a lower Ca conductance and a higher agonist affinity. Alpha7Rs enhance glut release and postsynaptic glut-mediated currents, and downregulate GABAA currents. Alpha4beta2 nAChRs are mostly located presynaptically on GABAergic IINs targeting pyramidal neu- 
rons [Albuquerque et al., 2009], but they also enhance glut release from thalamocortical neurons. In V1, ACh excites mostly sensory network (L4) neurons, via nAChRs [Lean et al., 2018]. Beta2-containing nAChRs directly excite deep layer pyramidal neurons and IINs [Poorthuis et al., 2013]. Alpha2-containing nAChRs are present in SOM CCN IINs that specifically target the response network [Hilscher et al., 2017], promoting competition resolution.

mAChRs are metabotropic and include two receptor families, M1/3/5, which are excitatory via $\mathrm{Gq}$ and $\mathrm{K}+$ channels, and M2/4, which are suppressive via Gi/o. Unlike the nicotinic receptors, which desensitize very quickly, the muscarinic receptors have a prolonged effect (but see [Muñoz and Rudy, 2014]). M1 mAChR is the most abundant in the brain. It is present in both glut and GABAergic neurons. The M2 family is primarily presynaptic, on both glut and GABA neurons. M1 mAChRs are coupled to intracellular signaling paths that turn on more slowly but have a longer impact than the M2/4 receptors.

We suggest that nicotinic receptors promote the disinhibition stage of competition while muscarinic receptors support the subsequent stage of prolonged focused execution. In both cases, ACh acts on both glut and GABA neurons. In the disinhibition stage, nAChRs first excite sensory inputs and IINs via alpha4beta2Rs, and as ACh levels increase they strongly excite glut neurons via lower affinity alpha7Rs. The excited IINs are connected by gap junctions and are fast spiking. In unautomated synapses, this gives an excitatory GABAergic initiation to glut neurons (ACh release should be minimal in automated synapses). Thus, the combined effect of nAChRs is to widen the disinhibition area. Indeed, Alpha7 disinhibits apical pyramidal dendrites, at the hippocampus [Albuquerque et al., 2009] and cortex [Letzkus et al., 2011], by exciting the VIP IINs in the CCN. Moreover, locomotion activates mouse V1 VIPs and downregulates SOMs, via BF ACh and nAChRs [Fu et al., 2014]. Since ACh uptake is very fast and nAChRs desensitize rapidly, this facilitation of disinhibition terminates at about the same time that competition is resolved by the CCN. Since sustained focused execution is acute, it involves a continuous release of ACh, so nicotinic receptors continue to support the focused disinhibited path.

In the consolidation and focused execution stages, the M1 family supports the winners while the M2 family terminates disinhibition and suppresses the losers. There is substantial evidence supporting this account. M4 suppresses the BG direct path and DA neurons, while M1 excites the BG indirect path [Ding et al., 2010]. ACh bursts suppress L1 NGFC IINs via mAChRs [Brombas et al., 2014], preventing the complete closure of the disinhibitory hole and thereby sustaining quax skeleton activity. In auditory cortex, mAChRs depolarize the response network, facilitating persistent firing, and suppress the internal networks [Joshi et al., 2016]. Muscarinic agonists powerfully depolarize SOM IINs [Fanselow et al., 2008], narrowing disinhibition to consolidate the winners. M1 enhances inhibitory GABA, while fast spiking of GABAergic neurons without M1 activation (corresponding to the competition stage) suppresses presynaptic GABA release [Domínguez et al., 2014]. In addition, mAChRs prevent the reduction of KCC2 (i.e., a more excitatory GABA) induced by repeated pre-postsynaptic spiking of GABAergic synapses [Mahadevan and Woodin, 2016].

Automated responses. After automaticity, ACh release is minimal but still topographic (similar to DA). The high affinity alpha4beta2 nAChRs support automated responses, e.g. by exciting CCN IINs to promote rapid competition resolution. Like DA, NE and SER, ACh utilizes suppressive autoreceptors (M2 mAChRs) to suppress ACh release after response execution, since positive feedback is not desired in automated responses.

$\mathrm{R}$ learning is not detailed in this paper. Nonetheless, it is worthwhile to briefly mention some learning aspects related to ACh. Alpha7 nAChR is essential for the GABA develop- 
ment switch from excitatory to inhibitory [Fernandes et al., 2014]. Neurogenesis, which reflects execution, is enhanced by M1 mAChR and decreased by nAChR [Van Kampen and Eckman, 2010]. There is massive ACh flux into the hippocampus during REM sleep, to promote the learning of previously executed quacia (see also the claustrum above).

BG cholinergic interneurons (CINs). The STR contains a population of cholinergic interneurons (CINs) comprising 1-5\% of the STR neurons [Gonzales and Smith, 2015]. They are innervated by the center median/parafascicular (CM/Pf) thalamic complex, which is the main source of thalamostriatal connections [Smith et al., 2014]. CM/Pf is innervated by projections from the reality network in low level object and motor areas.

The CINs are usually identified with the STR's tonically active neurons (TANs) and are assumed to provide constant MSN inhibition. In R17, their role is to promote transitions during focused and automated execution.

During alerts, surprises yield burst firing of thalamic CM/Pf neurons [Matsumoto et al., 2001]. The thalamic burst strongly excites the CINs, which burst to yield a strong pulse of suppression on ongoing direct MSNs via M2 mAChRs [Ding et al., 2010]. Following their burst, the CINs (and indirect MSNs) are paused via D2Rs and afterhyperpolarization. The $\mathrm{CIN}$ and MSN pause allows cortical, thalamic and amygdalar inputs to flow freely into the BG, creating a constraint-free path for competition candidates. After DA uptake, the CINs emerge from their temporary suppression to support indirect path activity via M1mAChRs [Ding et al., 2010], and gradually suppress the direct path. This explains the well-known excitation, pause, burst ('rebound') pattern exhibited by CINs, and also why an initial burst does not appear in many cases (surprises are smaller in everyday activity).

During automated execution, the CINs are still excited by thalamic inputs. In this mode there are no bursts, according with CIN tonic firing. CM/Pf-driven synchronous CIN activity triggers axonal DA release [Threlfell et al., 2012, Cachope et al., 2012], inducing a $\mathrm{D} 2 \mathrm{R}$ regime that facilitates quax transitions. The thalamic $\mathrm{Cm} / \mathrm{Pf}$ nuclei innervate the STN as well, promoting its support for the execution of responses to sensory inputs.

In summary, ACh supports $\mathrm{Q}$ process competition and automated responses via nicotinic receptors, and attended Q process execution via muscarinic (and nicotinic) receptors. Since Q process competition and execution are mediated by coordination networks, ACh is the main Rgen that drives cortical IINs. DA also promotes competition and execution, but it targets the $\mathrm{N}$ process, not the $\mathrm{Q}$ process, explaining why DA has a less pronounced effect on IINs and the complex relationship between DA and ACh in the BG.

\subsection{Pain, aggression, mating: substance $\mathbf{P}$ (SP), histamine}

Sensory pain [Basbaum et al., 2009] is triggered by nociceptors sensitive to possible tissue damage. The resulting signals are conveyed by dedicated (labeled) lines ${ }^{52}$ via superficial spinal dorsal horn laminae (lamina I for heat pain, lamina II for mechanical pain). Nociceptive inputs to the superficial dorsal horn express high levels of CP-AMPARs, yielding rapid excitation. Ascending pain flow reaches the lateral parabrachial nucleus, innate circuits (PAG, amygdala), thalamus, cortex (insula, mPFC/ACC), and the HT.

A large number of molecules is involved with pain transmission. Some pain inputs work directly via TRPV1 channels, and some are peptidergic. Two major pain peptides are substance $\mathbf{P}$ (SP) and calcitonin gene-related peptide (CGRP). CGRP excites pathways reporting pain stemming from neuronal inflammation [Iyengar et al., 2017], and SP and histamine report pain that affects the skin. SP signals via the neurokinin-1 receptor

\footnotetext{
${ }^{52}$ Although many nociceptors can be activated by several types of stimuli.
} 
(NK1R), which is excitatory via Gq, while CGRP is excitatory via Gs. SP and CGRP are expressed throughout the ibrain pain path. Most of the primary nociceptive inputs to the dorsal horn express SP, and most nociceptive lamina I neurons express NK1Rs. These are normally located close to SP neurons, allowing rapid and precise excitation.

Pain inputs normally trigger avoid innates, but SP also promotes adaptive responses. It is expressed in the HT and excites most LC NE neurons [Ebner and Singewald, 2006]. It is densely expressed in ACh areas, including the BF, lateral septum, and LDT projections to the cingulate cortices, and in the habenula MHb-IPN path (see below). Habenular SP projections to the DRN reduce SER via GABAergic DRN neurons [Zhao et al., 2015]. In the BG, SP is expressed in direct path neurons, CINs and the SNr, and NK1R is expressed by indirect path neurons [Mizutani et al., 2017]. NK1R (but not SP) is strongly expressed in the hippocampus. SP is opposed by the three opioid analgesics, BEND, DYN and ENK. NK1R is co-located with MOR (the BEND and ENK receptor) in the amygdala [PalomaresCastillo et al., 2012] (see ICMs below) and the MHb.

These data seem paradoxical. If SP communicates pain, it is natural to expect it to encourage adaptive actions meant to avoid pain. However, its effect seems to be the opposite, promoting a mode of execution ( $\mathrm{ACh}$ ) that involves attended interaction with objects. Attended execution actually reduces the perception of pain, and SP may mediate descending analgesia in the PAG [Drew et al., 2009]. Moreover, NK1R antagonists have only a limited effect on pain [Borsook et al., 2014].

We propose that the role of SP is to promote aggressive approach actions, including fight and mating. Fight and pain are obviously related, and fight and mating are related because both utilize intense charging approach innates, both involve rough touch, and animal mating attempts commonly involve aggression (male-male, male-female and femalemale). Indeed, stimulation of the intermediate HT area and the ventral VMH pole, which have high densities of NK1Rs, yields aggressive innates in male rats [Halasz et al., 2009]. Tachykinin (a SP homologue) promotes drosophila male-male aggression and male-female mating [Asahina et al., 2014], and NK1 knockout mice are less aggressive and have a higher SER tone [Nelson and Chiavegatto, 2001]. Moreover, the ARC KISS1 population inducing the acute release of sex hormones via GnRH and LH expresses tachykinin B, and peripheral administration of SP in men induces LH secretion [Fergani and Navarro, 2017]. SP is expressed in $\mathrm{BG}$ direct path MSNs because these are the neurons activated during charging responses to surprising objects, and the released SP acts to promote execution responses via CINs.

Histamine. Histamine [Haas et al., 2008] is involved in immune responses to inflammation. In the brain, it is released by neurons in the hypothalamic TMN. It operates via three receptors, $\mathrm{H} 1, \mathrm{H} 2$ and $\mathrm{H} 3$, which are GPCRs acting through Gq, Gs and Gi/o located in many brain areas, including the STR. Histamine neurons are excited by their adjacent OX neurons, and are classically thought to be tonically active and to promote circadian wakefulness, exhibiting high frequency firing in wakefulness and silence in sleep. Histamine excites motoneurons [Coslovich et al., 2017], depolarizes the superficial, but not the deep, MEC layers [He et al., 2016], excites NE and ACh, and affects SER. H3Rs promote/oppose D1Rs/D2Rs [Rapanelli, 2017].

These data show that like SP, histamine is involved in both innate and adaptive responses. Histamine neurons are excited by $\mathrm{C}$-fibers in lamina I spinothalamic neurons that are specific to conveying cutaneous itch (a non-histaminergic itch is triggered by the gastrin-releasing peptide receptor). Itch normally triggers an innate scratch response. His- 
tamine is analgesic ${ }^{53}$ in the PAG via H2R [Haas et al., 2008], again similarly to SP. We propose that histamine promotes innate and adaptive responses utilizing scratch actions. Indeed, histamine increases aggression [Nelson and Chiavegatto, 2001].

In summary, SP and histamine indicate urgent externally-induced needs, pain and itch, and promote efforts to resolve them. Probably due to the urgent and body-proximal nature of these needs, aggression is the preferred adaptive way to address them when innate avoid responses have failed. Since aggressive approach actions are also used by males during mating, SP has a role in mating. While sex hormones respond to innate sensory inputs, prime the sex organs and excite the brain mating paths, SP (and AVP) provide a link to the skeletal innate actions needed for mating-driven approach.

Additional important related topics are TD analgesia (see opioids below), termination via fight responses (see NOC below), and chronic pain (discussed elsewhere).

\subsection{Mistake: the habenula}

The habenula (Hb) [Benarroch, 2015] is a structure in the dorsomedial thalamus. It has two parts, lateral (LHb) and medial (MHb), containing glut neurons. LHb neurons express quickly excitable CP-AMPARs. LHb receives inputs from the PFC (ACC), anterior insula, BG, preoptic area, suprachiasmatic nucleus (SCN), HT, and the NE, DA and SER nuclei. There is a GABAergic BF population suppressing the LHb. LHb receives inputs from two BG populations, GP neurons ( $\mathrm{GPh}$ ) expressing glutamate and receiving input from the striosomes (see below) and cortex [Stephenson-Jones et al., 2013], and GABAergic VP neurons. LHb is suppressed by DA via D2Rs and via D4Rs, activated both by DA and by NE acting as a D4R agonist [Root et al., 2015]. SER excites the LHb via SER2c [Tchenio et al., 2016].

LHb innervates the rostromedial tegmental nucleus (RMTg), a VTA area containing GABAergic neurons projecting to VTA DA neurons [Jhou et al., 2009]. LHb innervates DRN and MRN SER neurons [Dorocic et al., 2014], and constitutes the major input to the DRN. It also targets histamine neurons.

LHb is activated in motivated negative situations [Proulx et al., 2014], especially in negative surprise (when the animal expects a reward but it does not arrive), errors, unexpected aversive events (airpuff), and noxious stimuli. In unrewarded trials, LHb excitation precedes the inhibition of DA neurons. LHb also promotes freeze.

$\mathrm{MHb}$ sends massive outputs to the interpeduncular nucleus (IPN). The IPN projects to the VTA, LDT, LC, and PAG, and is mutually connected by GABAergic projections with the MRN. MHb is mainly innervated by the septum and adjacent ACh nuclei (which provide it with ACh and GABA inputs), is bidirectionally connected with the MRN via GABAergic projections, and receives VTA DA and LC NE inputs. MHb and IPN have high MOR levels, which means that they are an adaptive TD path that utilizes innates (see BEND below). MOR is substantially colocated with SP at the MHb and IPN, so the utilized innates may be those supporting fight and mating. Selective ablation of $\mathrm{MHb}$ neurons induces hyperactivity and impulsivity.

Combining these data, the LHb and MHb are clearly associated with DA and ACh, respectively, to suppress decision and execution. More specifically, the habenula can suppress the final intense approach stage of execution (the charging promoted by DA, and the aggression promoted by SP in the ACh path). Both habenula parts are connected to a SER nucleus (DRN and MRN), whose role is to put a rapid brake on this final approach. We

\footnotetext{
${ }^{53}$ Which may explain why people continue scratching beyond what is necessary.
} 
propose that the role of the habenula is to rapidly stop execution to move to planning (nonurgent DM) mode. The switch from execution to planning is done when alerting sensory inputs indicate that executing the action would be a mistake (e.g., by contradicting the predictions for a positive valence driving the executing actions). This switch is what induces the well known DA dip in negative surprise [Tian and Uchida, 2015, Schultz, 2016]. The habenula is also involved in circadian sleep cycles, where it may be used as an efficient DA/ACh suppression tool.

\subsection{Consume: beta-endorphin (BEND)}

Needs and their manners of satisfaction are innate. Hence, final termination of adaptive responses is done via innates. A novel thesis of R17 is that $\mathrm{N}$ process termination via movement innates is mediated by the opioid (OP) peptides. There are four main OPs, betaendorphin, dynorphin, enkephalin and nociceptin, each facilitating certain innate actions and suppressing competing ones. The OPs are suppressive via GIRK channels and Gi/o, also retrogradedly (i.e., postsynaptic release suppressing presynapses), but can also excite cells via somatodendritic Cav2.1 channels [Fields and Margolis, 2015].

Beta-endorphin (BEND) belongs to the endorphin OP family. It is released by arcuate hypothalamus POMC neurons, some NTS neurons, and the pituitary. There are POMC projections onto the spinal cord, the LC, the parabrachial nucleus, and the PAG. Peripheral BEND may be able to enter the brain via the circumventricular organs lacking a blood-brain barrier (BBB) and via the choroid plexus (the blood-CSF barrier). BEND diffuses widely via the CSF, in the brain and the periphery [Veening et al., 2012]. BEND operates via muopioid receptors (MORs), densely located in key abrain-ibrain interface areas, including the STR (in striosomes, see below), VTA (RMTg), amygdala (ICMs, see below), medial habenula, and PAG. The PV and SOM IINs of the hippocampus express MOR [Volk et al., 2012]. In cortex, MOR is expressed in VIP IINs, many of which coexpress CRH [Taki et al., 2000].

Behaviorally, BEND is best known as an analgesic. MOR agonists such as morphine are extremely powerful pain relievers. This is implemented by MOR-induced suppression of GABAergic currents in the PAG-rostral ventromedial medulla (RVM)-spinal dorsal horn pathway [Fields, 2007]. BEND also counters alerts (stress) via strong innervation of the LC by HT POMC neurons, and by presynaptic suppression of thalamo-STR projections [Atwood et al., 2014].

BEND is strongly associated with pleasure, including food, sex and rhythmic music. HT POMC neurons directly sense body fat and blood glucose levels via leptin and insulin [Mercer et al., 2013]. NAc MORs increase fat, sucrose, salt, saccharin, and ethanol intake [Kelley et al., 2002]. There is a small NAc area in which MOR ligands yield increased innate orofacial licking ('liking') responses associated with food [Castro and Berridge, 2014]. NAc MOR antagonists attenuate palatable food intake, diminishing both liking and adaptive responses to sucrose ${ }^{54}$ [Shin et al., 2010]. CSF BEND rises during fat eating, then rapidly declines after 15 minutes [Mizushige et al., 2009].

Regarding copulation, lumbar spinothalamic MOR agonists can trigger ejaculation even without sensory stimulation [Kozyrev and Coolen, 2015]. HT POMC neurons have bidirectional connections with the HT KISS1 neurons driving acute sex hormone release [Backholer et al., 2010]. Estrogen yields BEND release from ARC to MPOA to promote lordosis, the innate female body posture for receptive copulation exhibited by most mammals

\footnotetext{
${ }^{54}$ The adaptive ('wanting') responses are measured by completion speed and distractibility on the way to a food reward.
} 
[Micevych et al., 2017]. Sex-related consume innates are promoted by another endorphin opioid, endomorphin, which strongly activates MORs [Paredes, 2014].

The association of BEND with dance and music may be related to its role in facilitating strong restraint-free copulatory and final preying movements. Active singing, dancing and drumming all trigger BEND release, while passive listening to music does not [Dunbar et al., 2012].

Striosomes. MSN protein expression can be used to divide the STR into two areas, striosomes (patch) and matrix [Crittenden and Graybiel, 2011]. Patch MSNs express high levels of MORs, SP, D1Rs, and calretinin, while matrix MSNs express calbindin, SOM, and ACh markers, and show higher level of D2R than D1R. MOR-expressing MSNs are located in patches whose cortical innervation is mostly from valence areas (OFC, ACC, insula, amygdala). The thalamic nucleus supporting valence, PVT, prefers the striosomes [Unzai et al., 2017]. In DA nuclei, patch MSNs project to DA neurons while matrix MSNs project to GABAergic IINs [Fujiyama et al., 2011, Watabe-Uchida et al., 2012]. DA neurons densely innervate the patches, and patch neurons are first to develop. The GP glut population projecting to the $\mathrm{LHb}$ receives input only from striosome MSNs [StephensonJones et al., 2013]. By their molecular markers and innervation patterns, the striosomes clearly support actions that are strongly valence-driven.

RMTg. The GABAergic RMTg neurons projecting to VTA DA neurons express high level of MOR [Jhou et al., 2009]. Thus, in both the striosomes and the VTA, BEND upregulates DA by suppressing GABAergic neurons that suppress it. In addition, MOR directly excites VTA DA neurons [Fields and Margolis, 2015].

ICMs. The amygdala has a few small GABAergic nuclei called the intercalated masses (ICMs) (see Figure 3). One ICM island passes flow from mPFC to BLA, others from BLA to $\mathrm{CeA}$, and another from infralimbic PFC to CeM. Based on flow directions, the ICMs have a TD (adaptive) function. The ICMs are densely populated with MORs, and (at least the main BLA-CeA island) are enriched in D1Rs and ENK that are expressed in different sub-regions [Jacobsen et al., 2006]. Amygdalar D1Rs are most abundant in the ICMs. Amygdalar flow, including that passing through the ICMs, is generally unidirectional (sensory input/mPFC-BLA-CeM-PAG).

Combining these data, a striking similarity of the ICMs and the striosome (which contains direct D1 and indirect ENK MSNs just as the STR matrix) is evident ${ }^{55}$. Supporting the involvement of ICMs in TD adaptive valence-driven responses, there are very dense posterior OFC projections to the CeM-projecting ICM, which are suppressed by alerts [Zikopoulos et al., 2017].

We note that there are many ICMs, and it is possible that not all of them express MORs. The role of such ICMs, if they exist, can be deduced from their Rgen expression.

Function. To understand the role of BEND in the N process, consider the following points. First, BEND acts exactly at the time point in which needs start getting satisfied, and POMC neurons receive signals to that effect (by direct sensing of food, and innervation from KISS1 neurons that sense sex hormone levels). Second, BEND seems to facilitate innate consume responses associated with need satisfaction (licking, ejaculation). Third, in the amygdala, BEND prevents TD flow from reaching the innate control area, CeA, and in the BG, BEND suppresses adaptive flow supporting strong valenced actions. Fourth, analgesia is achieved via suppression of a TD path (PAG-RVM-dorsal horn). Fifth, in the MHb, MOR suppresses

\footnotetext{
${ }^{55}$ A general ICM-STR similarity has already been noted [Palomares-Castillo et al., 2012], but not with respect to striosomes, D1 and ENK.
} 
the attended execution path.

These data imply that BEND promotes innate consume responses and disconnects adaptive responses. However, BEND upregulates DA, which facilitates an adaptive mode, not an innate one. An answer to this puzzle comes by noting that MOR promotes innate responses to cues predicting reward. For example, BLA MORs facilitate vigorous approach to predictive food cues [Lichtenberg and Wassum, 2017]. Cues are learned by conditioning, so are part of the innate+ system, which extends the innate system. Thus, BEND promotes DA to support rapid execution of innates, the final charging actions before consummatory acts.

We conclude that the role of BEND is to promote immediate innate consume responses that utilize external movement. BEND-releasing neurons receive both predictive signals indicating imminent satisfaction and innate signals indicating the start of satisfaction. The resulting BEND release promotes strong adaptive responses leading to consume innates (via D1), and the consume innates themselves (via the PAG-spinal dorsal horn path). After this initial boost, BEND levels decrease and it slowly diffuses all over the brain, where its inhibitory effect yields relaxation of adaptive responses. Innate stimulus-driven responses continue for some time, but with reduced vigor ${ }^{56}$.

BEND is analgesic because as in all brain execution, quax formation involves competition, and BEND suppresses the losers. Since the BEND-promoted responses are implemented at the spinal level, their competitors are other spinal paths, including ascending spinal pain pathways. These are active because successful predation virtually always involves fight. Pain promotes avoid and aggression innates, so suppressing it allows other types of innates. Indeed, MOR-induced analgesia classically involve MOR-activated cells that suppress responses to noxious stimuli ('OFF cells') and MOR-suppressed cells activated by such stimuli ('ON cells') [Fields, 2007].

BEND seems to act specifically on success-related responses that utilize skeletal muscles, while success responses that involve smooth muscles are mediated by a different agent (OT, see below).

Striosomes, dopamine. Returning to the striosomes, it should be asked when exactly they are used and why their MSNs project directly to DA neurons while matrix MSNs project to DA nuclei IINs. As noted above, the striosomes support actions strongly driven by valence. This includes conditioned cue-driven (i.e., innate+) actions, and actions in which termination by innates is imminent. Indeed, the BG allow locomotion, posture, mouth movements, vocalization, and saccades innates when executed in adaptive mode [Hikosaka, 2007].

Surprising cues yield a D1 regime that switches to a D2 regime when using learned transitions. In matrix-supported actions, the competition (D1) stage is followed by sustained execution of adaptive actions. The coordination used by such actions utilizes GABAGABA-content paths (a VIP-SOM-pyramidal neuron path for representations, and a MSNVTA IIN-DA path for DA supply). In striosome-supported actions, competition is followed by charging innate execution, so the duration of subsequent adaptive transitions is very short. Indeed, the number of patch D2R MSNs is small. Since coordination of DA is not needed, when activated, patch MSNs directly suppress DA release.

The sequence of events in this account is as follows. First, an innate+ cue excites BLA representations. These activate cortical valence nodes, POMC neurons, and (mostly) patch

\footnotetext{
${ }^{56}$ This explains why eating can be largely detached from energy needs, and why when engaging in a pleasurable activity (eating, listening to music) it is not easy to sustain attention to it. Normally, people continue eating or listening in an automated manner while their thoughts wander or while conversing with others.
} 
MSNs. The activated nodes and POMC-released BEND promote phasic DA release (the DA surge in the classical reward prediction error models). The resulting D1 regime allows STR competition, which takes place in the striosomes because these are the MSNs innervated by imminent innate valence flow. At the same time, BEND mediates competition in the ibrain. The activated striosome MSNs suppress the $\mathrm{GPh}$ population, preventing the $\mathrm{LHb}$ from inducing its rapid brake on approach actions. When the competition resolves, the BG allow adaptive charging actions, the appropriate innates start executing, and the striosome MSNs directly suppress DA. The execution of innates yields satisfaction, which increases POMC firing and BEND levels. After some time (usually after the action is over), BEND diffusion suppresses TD execution via its actions in the striosomes, MOR-expressing ICMs, $\mathrm{MHb}$ and the ibrain, allowing more relaxed sensory-driven innates.

In summary, BEND strongly amplifies skeletal consume innates and DA, and then suppresses valenced responses. Thus, it promotes what is usually called hedonic pleasure, the uncontrolled, frenzied and strongly satisfying response at the initial stage of need satisfaction, and assists subsequent unstressful relaxation.

\subsection{Satisfaction: oxytocin (OT)}

Oxytocin (OT) [Stoop, 2012] is a neuropeptide very closely related to AVP. It is released peripherally and by PVH and supraoptic HT neurons, using both synaptic and somatodendritic release. Its half life is short, about 20 minutes. The OT receptor (OTR) is a GPCR that can be excitatory or inhibitory by binding to Gq, Gi/o and Gs. OTR is expressed mostly in valence areas, including the amygdala CeL, BNST, mPFC, olfactory bulb, NAc, hippocampus, lateral septum, LC, other HT nuclei, and the spinal cord. Like BEND and AVP, OT can diffuse widely via the CSF [Veening et al., 2010].

Innate and need effects. OT is critical for peripheral processes at the time of birth, including facilitation of parturition, lactation, and maternal behavior. It is present in human milk [Carter, 2014], and can be released via the activation of oral mucosa and the gastrointestinal tract [Uvnäs-Moberg et al., 2014]. These situations are also reported to the brain via the NTS. It is also released in response to activation of $\mathrm{C}$ fibers that mediate non-painful and pleasant information, e.g., light touch, stroking, and warmth. Pleasant touch activates hypothalamic OT neurons, and yields $50 \mathrm{~Hz}$ vocalizations, indicating positive affect, in rats [Okabe et al., 2015].

OT is also involved in sex innates [Veening et al., 2015]. The female pelvic copulatory organs express OTRs, which can provide lubrication, muscular contractility and analgesia. OT facilitates female lordosis and male ejaculation. It also excites HT POMC neurons [Maejima et al., 2014].

OT terminates stress, both peripherally and in the brain, and generally reduces food intake. Anterior pituitary OT release inhibits $\mathrm{ACTH}$, and circulating OT directly inhibits adrenal GC release [Uvnäs-Moberg et al., 2014]. OT suppresses stress-induced increase in PVH CRH via GABAARs [Smith et al., 2016], and is highly cardioprotective [Gutkowska et al., 2014]. OT is expressed in more than half of the CeL PKCD neurons supporting innate paths [Haubensak et al., 2010], and reduces conditioned freezing but not cardiovascular response [Viviani et al., 2011]. OT yields the anxiolytic effect of post mating in males [Waldherr and Neumann, 2007].

OT is essential for the GABA switch after birth, downregulating NKCC1 [Ben-Ari, 2014]. This accords with OT and AVP having opposite effects, because bumetanide, a well-known diuretic (countering AVP), works by blocking NKCC1. AVP and OT signal a need (for water, sex) and its resolution, respectively. 
Sociality. Many, perhaps all, of the innate effects of OT occur in behaviors that involve interaction with conspecifics. Since OT signals success and subsequent pleasant relaxation, it promotes positive (approach) innate+ responses to conspecifics. A large body of work supports this role of OT in sociality [Harari-Dahan and Bernstein, 2014] (see also language below). However, since OT mediates a wide range of approach innates and works in conjunction with other Rgens (see DA below), it can also promote non-social approach responses and 'paradoxical' negative responses. Indeed, exogenous OT can promote intergroup discrimination and defensive aggression towards out-of-group individuals [De Dreu and Kret, 2016]. These are easily explained by OT's role in the N process, since suppression of acute adaptive responses (attention, control) facilitates innate responses (including negative ones), and the excitation of the conspecific representation paths may select aggression responses.

Rgens. OT cooperates with DA in its approach effects. Injection of OT into the VTA, and mother licking and grooming, increase NAc DA levels [Shahrokh et al., 2010]. About half of the VTA OTR-expressing neurons express glut but not DA [Peris et al., 2017], providing a way for OT to directly excite DA neurons. Some of the relaxation effects of OT are mediated via OTRs on SER raphe neurons. For example, SER is involved in its affective effects in touch [McGlone et al., 2014], and social reward requires the downregulation of NAc neurons via OT-mediated SER1B [Dölen et al., 2013]. OT also uses the cannabinoid CB1 receptor for termination [Ninan, 2011].

$\mathbf{N}$ process role. OT is similar to BEND in many ways. Both signal immediate success and longer term relaxation, are released just before satisfaction to facilitate it, promote DA, reduce pain, distribute through the CSF, and disconnect adaptive responses via the BG. The difference between them seems to be that the innates promoted by BEND use skeletal movements, while OT affects smooth muscles and the secretion of fluids. OT is also associated with movements, but these are delicate ones (light touch, caress) at the receiving end, while BEND promotes frenzied movement execution. Because it conveys the positive valence associated with conspecifics, OT is critical for social learning.

\subsection{Failure, withdrawal: dynorphin (DYN)}

The opioid dynorphin (DYN) [Tejeda et al., 2012] signals through kappa opioid receptors (KORs), which are suppressive via Gi/o and calcium and $\mathrm{K}+$ channels. It is expressed in ibrain areas, many of the amygdala CeA CRH-expressing neurons, mPFC-projecting BLA neurons, almost all HT OX neurons, HT AVP neurons, ARC KISS1 neurons, and direct path BG MSNs. It is released by intensely active neurons and suppresses their inputs in a retrograde manner.

Like BEND and the other opioids, DYN has strong innate effects and suppresses adaptive execution. It is analgesic, but not as strong as BEND. There is extensive evidence that it promotes freeze and withdrawal innates. For example, amygdala CeL neurons expressing DYN mediate innate freezing [Jüngling et al., 2015]. According with freeze, DYN reduces blood pressure and heart rate [Guo et al., 2007]. It is increased by acute social defeat stress [Donahue et al., 2015], mediates social avoidance [Resendez et al., 2016], and is strongly associated with depression and the dysphoric states resulting from addiction [Van't Veer and Carlezon, 2013, Tejeda et al., 2012].

The CRH neurons that express DYN project to the VTA, SNc, and to LC NE neurons [Reyes et al., 2011]. DYN suppresses DRN SER neurons that project to the vSTR [Schindler et al., 2012]. DYN opposes basal forebrain ACh [Ferrari et al., 2016], and colo- 
calizes with SP in BNST, MPOA, CeM. KORs are expressed by HT AgRP neurons. Thus, DYN suppresses the acute mode (NE, CRH, AVP, OX, DA, AgRPs), execution (ACh, SP), and motivated decision making (SER). In other words, it suppresses adaptive responses.

Examining this data, it is evident that like the other OPs, DYN promotes termination by innates. The innate response that it promotes is freeze/disengage, which provides analgesia via competition with pain pathways. Through its innate role, DYN terminates adaptive responses in case of failure. DYN is released when the direct BG path has been active for an extended period, implying a prolonged acute decision making. This happens when the brain does not manage to find a way to deal with an urgently alerting situation. Thus, the role of DYN is to promote withdrawal and switch adaptive responses off. This response is protective in two ways, avoiding the alert itself, and preventing cell damage (e.g., oxidative stress) due to prolonged intense activity. This novel R17 account explains DYN's involvement in psychiatric disorders, detailed elsewhere.

\subsection{Aggression: nociceptin (NOC, N/OFQ)}

Nociceptin (NOC) [Toll et al., 2016] (also called nociceptin/orphanin FQ (N/OFQ)) is a recently discovered opioid acting through the NOP (nociceptin/orphanin FQ opioid peptide) receptor. NOP is widely expressed in innate and valence areas, including the superficial dorsal horn laminae I and II conveying nociceptive inputs, PAG, CeA, HT VTA, NAc, $\mathrm{PFC}$, the lateral septum, MHb, and the BNST. More than half of BNST neurons express NOP [Kash et al., 2015].

NOC suppresses the other OPs, allowing innates that they suppress. Specifically, it is not analgesic. Spinal NOC induces scratching, biting, and licking responses via NK1, the SP receptor [Sakurada et al., 1999]. In the HT, it is expressed in nuclei promoting aggression (MPOA, VMH and SUM). Social defeat increases STR NOP [Der-Avakian et al., 2017]. Structurally, it is related to DYN, the other OP associated with negative innates. NOC also promotes micturition, prevented by combined NK1 and NK2 antagonists [Lecci et al., 2000]. This innate response is common in alert situations, here probably assisting fighting.

In summary, although the amount of available data is limited, it seems that like the other OPs, NOC promotes termination via a basic innate response. By its association with SP, its distribution, and its behavioral effects, this innate response is most likely fight.

\subsection{Running: enkephalin (ENK)}

The opioid enkephalin (ENK) signals through delta opioid receptors (DORs) and MORs, and is suppressive via Gi/o, mostly retrogradedly. It is expressed in many brain areas, including the spinal dorsal horn (particularly in laminae I and II), PAG, the parabrachial nucleus, the NTS, and medullar nuclei innervating the LC, amygdala ICMs and CeL (where DYN/CRH and ENK neurons are different populations), cortical motor areas, and BG indirect path MSNs. It is colocalized with CRH in many hypothalamic neurons and the BNST [Valentino and Van Bockstaele, 2015]. Like BEND and DYN, it is analgesic.

In addition to being a DOR ligand, ENK is a MOR ligand, and like BEND there is a STR ENK surge when eating palatable food (chocolate) [DiFeliceantonio et al., 2012]. ENK suppresses excitatory inputs to the STR, preferentially cortical ones, while MOR prefers thalamic ones (according with their tight connection with sensory inputs) [Atwood et al., 2014]. Like DYN, it is released after prolonged cell activity and suppresses OX [Li and van den Pol, 2008]. 
These data support a role for ENK as a protection agent that quenches prolonged focused execution, especially during motor execution but also during consume innates (being a MOR ligand). Like BEND and DYN, it promotes termination via innates, with a focus on locomotion innates. Indeed, DORs have well-known neuroprotective and analgesic effects [Feng et al., 2012] and increase locomotion [Katsuura and Taha, 2010]. Since ENK promotes running, analgesia, and MORs, it is a prime candidate for mediating the feeling known as 'runner's high', classically thought to involve endorphins ${ }^{57}$.

\subsection{Interaction: melanin-concentrating hormone (MCH)}

Melanin-concentrating hormone (MCH) [Brown et al., 2015] is released by LH and zona incerta neurons generally considered to be GABAergic (although some express glut). The brain has two MCH receptors, MCHR1, which is a GPCR that activates Gi/o and Gq, and MCHR2 (not present in rodents), which activates Gq. The two receptors have a similar brain distribution and show a high affinity to $\mathrm{MCH}$. MCH neurons project widely throughout the brain, most strongly to alert/acute areas (LC, amygdala BLA, hippocampus, NAc shell, VMH, ARC). In the BG, MCHR1 is expressed on both paths in the NAc shell, reducing MSN excitability. $\mathrm{MCH}$ neurons receive strong amygdala and BNST inputs. $\mathrm{MCH}$ opposes need and alert agents (OX, CRH, NE, histamine), and also DA and some DRN SER neurons. $\mathrm{MCH}$ is also suppressed by the negative termination Rgens DYN and NOC. $\mathrm{MCH}$ increases plasma levels of ACTH, which promotes energy management (below). According with these relationships, MCH is anxiolytic [Kela et al., 2003].

Behaviorally, $\mathrm{MCH}$ has two main known effects, promotion of feeding and of sleep. With respect to feeding, MCH neurons are directly excited by glucose. MCHR1 antagonists reduce feeding, meal size and weight gain in normal weight and obese rodents [Georgescu et al., 2005]. Central administration of MCH increases water intake. There is some evidence relating $\mathrm{MCH}$ to olfaction, which is linked to food [Adams et al., 2011]. In rodents, amygdala-MCH projections are from olfaction nuclei [Niu et al., 2012]. MCH KO mice have significant difficulty in locating hidden food. In addition, $\mathrm{MCH}$ KO mice are impaired in olfaction-mediated behavior such as mating, inter-female estrous cycle synchronization and maternal behavior. $\mathrm{MCH} \mathrm{KO}$ males are excessively aggressive.

With respect to sleep, there is an $\mathrm{MCH}$ population that promotes REM sleep via projections to specialized sleep nuclei. The effect of $\mathrm{MCH}$ seems to be specific to promoting REM sleep [Vetrivelan et al., 2016]. MCH neurons fire maximally (3-12 Hz) during REM sleep, their activation during NREM sleep induces a transition to REMS, and their highfrequency stimulation during REMS prolongs its duration. They are not essential for sleep.

Combining these data, we propose that the role of $\mathrm{MCH}$ is to quickly terminate alerts to promote interaction with objects, especially those associated with approach actions (feeding, mating). It is excited by amygdala and BNST inputs indicating non-negative stimuli. Indeed, $\mathrm{MCH}$ neurons were recently shown to be activated during non-stressful novelty [González et al., 2016a], and human amygdala MCH is minimal during emotional situations [Blouin et al., 2013]. After its release, it has a strong short term alert quenching effect, and also promotes longer term termination via GCs. MCH has completely opposite effects to those of its LH neighbor OX. OX neurons are glucose-inhibited and signal alerts, while $\mathrm{MCH}$ neurons are glucose excited and cancel alerts.

$\mathrm{MCH}$ has a role in sleep because it is memory promoting and participates in the learning process, probably via the binding of $\mathrm{MCH}$ receptors to Gq (see below). REM sleep is the sleep stage at which learning occurs, explaining the specific role of $\mathrm{MCH}$ in REMS. MCH

\footnotetext{
${ }^{57}$ ECBs are also strong candidates [Fuss et al., 2015].
} 
promotes GCs and ACh efflux into the hippocampus [Lu et al., 2013], and both of these are important factors in REMS learning.

An apparent paradox is that although MCH cancels alerts, $\mathrm{MCH}$ antagonism is considered to be anxiolytic [Lee et al., 2011]. This can be explained by examining the paradigms used to measure anxiety. Lee et al used the established elevated plus maze and light-dark transition paradigms, where entry into open arms or the light compartment indicates reduced anxiety. However, this measure reflects the extended alert $\mathrm{N}$ mode more than it reflects the interaction mode, because an elevated open arm is a dangerous situation that should induce slow movements, and there is no object to trigger interaction. Antagonism of $\mathrm{MCH}$ removes its opposition to $\mathrm{CRH}$, thereby promoting vigilant adaptive responses (rather than innate freezing). Similarly, $\mathrm{MCH}$ was reported to produce a depressive effect in the swim test [Georgescu et al., 2005]. In all of these cases, the alerts that MCH opposes are needed for responding, so its antagonism allows responses and therefore seems anxiolytic. These paradigms measure the animal's capacity of forming adaptive responses to counter innate freezing in stressful situations, reflecting the paralyzing aspect of anxiety, but they do not reflect well the difference between situation- and stimulus-induced alerts.

\subsection{Energy, protection: glucocorticoids (GCs), endocannabinoids (ECBs), DHEA}

Execution requires energy. Prolonged acute execution is costly in terms of energy, and is dangerous to cells due to oxidative stress and related processes. Hence, the $\mathrm{R}$ process has an energy management mode that provides energy and protects cells via a negative feedback mechanism. The latter suppresses acute responses after excessive execution (typically minutes to dozens of minutes). This promotes innate responses without promoting any specific innate.

Energy is supported by a complex metabolism system, described elsewhere. Here we describe three Rgens that are directly involved in the R process, glucocorticoids (GCs) (or corticosteroids, cortisol in humans and corticosterone in rodents), endocannabinoids (ECBs), and dihydroepiandrosterone (DHEA). GCs and DHEA are steroids. GCs and ECBs are strongly involved in energy metabolism in many body organs [Balsevich et al., 2017]. The peripheral role of GCs is to maintain blood glucose levels by promoting glycogenolysis, gluconeogenesis (mostly in the liver), and lipolysis, and by reducing glucose uptake.

GC and DHEA release is initiated by hypothalamic CRH that stimulates pituitary ACTH, which in turn stimulates steroid release from the adrenal cortex [Joëls et al., 2012] to form the well-known HT pituitary adrenal (HPA) stress axis ${ }^{58}$. The HT triggers tonic steroid release in fixed basal rhythms (circadian and hourly), and there is greatly increased release in the extended alert mode. GCs are small molecules that easily cross the blood-brain barrier to act in the brain. They act via two receptors, MR and GR. The affinity of MR is ten-fold higher than that of GR. MR and GR are expressed in neurons and glia. GR is expressed all over the brain with a dense expression in valence areas. MR is expressed in valence areas, PFC and the hippocampus. Again like the sex hormones, GCs have genomic long term effects mediated by nuclear receptors and faster non-genomic effects mediated by membrane and genomic receptors [Myers et al., 2014]. Most of the acute short term effects of GCs are done via ECBs.

The ECBs [Mechoulam and Parker, 2013, Balsevich et al., 2017] are a large family of

\footnotetext{
${ }^{58} \mathrm{~A}$ similar process induces the release of gonadal sex hormones, see above.
} 
agents, the two main ones being AEA ( $\mathrm{N}$-arachidonoyl ethanolamine, also known as anandamide) and 2-AG (2-arachidonoyl glycerol). 2-AG is primarily a retrograde messenger, released by neurons in dendritic compartments to bind to presynaptic receptors. Its degradation is presynaptic. AEA is synthesized both pre- and postsynaptically and is degraded postsynaptically, so it can act through both retrograde and anterograde signaling. The main ECB receptors are $\mathrm{CB} 1$ and $\mathrm{CB} 2$, operating in the brain and the periphery, respectively. ECB receptors are GPCRs that suppress neurons and agent release via Gi/o, inhibition of calcium channels, and stimulation of GIRK channels. AEA has higher affinity at both receptors, and it also directly increases glut release probability via TRPV1 channels.

The non-acute (basal) effects of ECBs and GCs are mediated by the higher affinities $A E A$ and $M R$, while the acute effects are mediated by 2-AG and GR. In non-acute conditions, GCs facilitate glut transmission via a presynaptic MR-mediated effect [Karst et al., 2010], and there is a tonic AEA tone (e.g., in the amygdala). GCs rapidly stimulate GluA2containing CI AMPARs (i.e., the glut receptors involved in automated responses) via MRs [Groc et al., 2008]. Acute neuronal firing yields postsynaptic synthesis and release of 2-AG, which binds to presynaptic CB1Rs to quench transmission. 2-AG synthesis is commonly induced by GCs. In the BG, CB1Rs are highly expressed on cortico-STR synapses [Shen et al., 2008], MSN axons, and IINs [Rossi et al., 2008]. In cortex, CB1Rs suppress the execution and competition coordination networks, being present in most cortical CCK, SOM, and VIP IINs, and also in half of the pyramidal neurons [Hill et al., 2007]. Presynaptic CB1Rs are abundant in the basket CCK IINs of the competition coordination network, with relative paucity in the execution coordination network. This is explained by noting that the latter network supports both automated and acute execution. Thus, GCs suppress acute responses via GR, reducing both excitatory and coordinating transmission in cortex and the BG. In addition, GCs suppress their own release via ECB-mediated suppression of HT CRH neurons [Uchoa et al., 2014]. In other words, GCs utilize two ways to provide negative feedback on acute responses, in order to protect cells from excessive activity-induced damage.

GCs do not induce brain-wide response suppression, which would be dangerous when responses are required. In valence areas (amygdala CeA, BNST, hippocampus, OFC), GCs yield a rapid excitatory effect [Schulkin, 2017], and alerts reduce AEA (the non-acute ECB) via CRH1R-mediated increase of its degradation by FAAH. The GC-mediated excitation is sustained for a prolonged duration in the amygdala and ventral hippocampus [Joëls et al., 2012, McEwen et al., 2016]. The combined short term effect of GCs and 2-AG is thus to suppress acute execution and promote automated and innate responses.

DHEA is the precursor of the steroid sex hormones. DHEA and GCs oppose each other, both in synthesis and in brain effects [Kamin and Kertes, 2017]. Unlike GCs, which are only released by the adrenal cortex, only about $80 \%$ of DHEA is released by the adrenal cortex, with the rest synthesized in mitochondria of brain cells ${ }^{59}$. DHEA does not have a negative FB mechanism. DHEA weakens GABAergic transmission, enhances NMDA transmission, and is the precursor of estrogen, which substantially optimizes brain energetic execution efficiency and health [Brinton, 2008].

In light of this, we propose the following novel view of GCs and DHEA. The role of DHEA in the brain is to promote execution by optimizing the energy utilization of the quax skeleton. This is a continue energy management $\mathrm{R}$ mode. The role of GCs is to suppress acute execution, constituting a protection energy management mode $e^{60}$.

\footnotetext{
${ }^{59}$ Neurosteroids are discussed in depth with plasticity elsewhere.

${ }^{60} \mathrm{The}$ long term learning (plasticity) effects of energy agents, as well as the detrimental effects of chronic release, are discussed elsewhere.
} 
Protection offers an explanation of various known phenomena related to stress and ECBs. Stress and GCs impair memory retrieval, which is a short term non-automated adaptive process, but enhance long term emotional (i.e., valence) memory consolidation and extinction [Roozendaal and McGaugh, 2011]. Cannabis, which contains several ECBs, can relieve stress and pain as part of suppressing acute execution, but it is also known to induce panic attacks, since these are an innate response, and to impair memory ${ }^{61}$.

According to R17, all life systems are managed by an R process, and agents participating in several systems should have similar roles in these systems. Indeed, in the immune system, GCs have a major role in suppressing inflammation, which is the immune parallel of the brain's acute response. However, they can also enhance innate inflammatory responses [Cain and Cidlowski, 2017]. This apparent paradox is easily explained by noting that this is exactly what GCs do in the brain. This is a good example of how an R process analysis can improve our understanding of other living systems.

\section{Language}

Language utilizes the same Q process as motor control, but it has some unique aspects that we highlight in this section. Much of the discussion pertains to inter-animal communication in general.

\subsection{The nature of language}

The brain's function is to generate movements to satisfy needs. Our view is that like other brain capacities, communication is a way of generating movements. It differs from other capacities in that the generated movements are made by other organisms. Our everyday use of language may hide this nature, but this is what language is from the brain's perspective.

The organisms at the recipient side of language produce movement via their brains. Hence, communication needs to activate neurons located in others' brains. Due to the lack of a direct neuronal connection between the two brains, a bridging medium (auditory, touch, visual) must be used. Audition is an effective medium in natural settings, because it can operate over long distances and is omnidirectional (it does not require narrow spatial attention). Human language is a communication capacity that uses sounds to bridge the inter-organism neuronal gap. Apart from this difference, language uses the same brain processes used by other capacities.

\subsection{Representations \& areas}

Content types. Language involves three types of content. The first includes the needs that drive communication and the sensory events that would satisfy them. This content type is the meaning of the linguistic utterance. The second includes the sounds (or gestures, or written symbols) used for bridging the neuronal gap. This content type is the form of the linguistic utterance. The third type comprises the interface between meaning and form. This language-unique type is needed because something needs to map goal attainment in the lower level auditory motor nodes to that in the high level meaning nodes.

\footnotetext{
${ }^{61}$ Cannabis also induces hunger, via feeding-promoting ECB actions in feeding areas designed to replenish energy [Cristino et al., 2014]. Again, the full R17 account of metabolism is give elsewhere.
} 
The first two types roughly correspond to the classical linguistic areas of semantics and phonetics / phonology. The third type requires some discussion. First, it includes the classical linguistic notions of lexicon (vocabulary) and syntax (grammar). Modern linguistics has recognized that words and grammatical patterns are similar in that they both interface between form and meaning, and has unified them in the notion of the linguistic construction (or unit) [Goldberg, 2006, Langacker, 2008]. We refer to this form-meaning interface content type as lexicon-syntax (LS). LS spans a continuum between simple morphemes (parts of words that carry meanings, e.g., morphological affixes such as 'un-'), words, multiword expressions, and detailed and abstract grammatical patterns.

There is a second content type connecting meaning to form. This type includes all of the knowledge that the person has acquired through language and that requires form-meaning mediation in order to be used. For example, the fact that Paris is the capital of France is known to people only via interactions that use language. We use the word knowledge to refer to this content type, since this is what it usually means in everyday use. Together, the two form-meaning types are referred to as LSK linguistic content.

Words. To yield movement by the recipient, language should be capable of invoking goals in the recipient's brain. Since goals are represented by configurations of object nodes, language needs to be able to describe objects and object configurations. Describing objects is the role of words, an LS object type. Objects strongly grounded in sensory inputs are described by so-called concrete words (e.g., 'woman'). Language can also refer to a shared aspect of configurations that have very few concrete sensory features in common, using abstract words. For example, the word 'love' can refer to very different situations (compare loving your child vs. 'I love apples'), but they all share the important quality of involving positive approach innates.

Syntax. The number of object configurations is too large to be able to represent all (or even most) of them through words, so multi-word utterances are essential for language. Syntax (commonly called 'grammar') manages the usage of word sequences to express meanings that are not expressed by individual words. Its main tools are word order, word inflections, and auxiliary words such as prepositions, all denoting the role of words in the larger utterance. Like words, there are very specific grammatical patterns (e.g., the unique word order in sentences that use the word 'ago'), and abstract grammatical patterns (e.g., that the verb agrees in number with the subject in Hebrew). Syntax is thus used to assemble word sequences in a similar manner to how sequences of low level motor actions are used to attain higher level goals.

Areas. The three content types are represented in different brain areas. Semantics areas include areas representing needs (mPFC, ACC, the anterior insula), high level action plans (the frontal pole, dlPFC), valence (OFC, the amygdala), physical space (parietal cortex, the hippocampal formation), and specific sensory modalities (occipital, somatosensory, posterior insular cortices). In other words, they include all of the brain areas that are not language-specific.

Auditory areas consist of representations supporting the audition part of language. These are sounds and self movements that acquire language specific use. Thus, the auditory areas include low level motor areas interfacing with the speech organs (including the cerebellum), and the auditory cortices and areas close to them in the postero-dorsal auditory stream (planum temporale (PT), temporo-parietal junction (TPJ), and inferior parietal (IP) cortex). Language form representations are also present in non-auditory areas, e.g., to support motor gestures that a person uses to accompany speech. 
LSK areas are organized along dorsal and ventral streams ${ }^{62}$ supporting context dependent and context independent content respectively. The dorsal LS areas are located on the strip extending from PFC in the direction of the auditory cortex (i.e., along the Sylvian fissure (lateral sulcus)). This strip includes syntax areas, which are laterally adjacent to premotor cortex (PMC) and are traditionally called Broca's area (approximately BA 44 and 45 , left inferior frontal gyrus (LIFG) ${ }^{63}$ ), and reaches the lexicon area (below). Note that PMC supports sequences of basic actions, which is the exact analogue of syntax, as noted above. The frontal areas supporting language, BA 46, 45 and 44 (anterior to posterior), have a semantic, syntactic, and auditory-syntactic nature, respectively. Indeed, BA 44 is dysgranular, according with quick auditory transitions [Zilles and Amunts, 2018]. The cerebellum specializes in rapid quax transitions, so it supports both audition and syntax [Mariën et al., 2014].

The context-independent linguistic objects are called words, phrases, concepts, facts, etc. and are located more ventrally in the posterior and anterior temporal lobe (the adjacent more posterior fusiform area represents context-independent visual objects, and is usually more prominent in the opposite hemisphere). The words area is traditionally called Wernicke's area (BA 22). Knowledge is located in the anterior temporal lobe (ATL), and is obviously tightly connected to relevant representations acquired through other means. For example, linguistically acquired information about people (name, occupation etc.) is represented at the ATL along with their faces [Wang et al., 2017], which are visually acquired ${ }^{64}$. If the interaction with a person strongly involves other modalities, the main association area representing that person would be located elsewhere. For example, interaction with very close people involves interoception, so their representations are more medial [Fiacconi et al., 2014].

\subsection{Processing}

The process. As with all actions, language processing is performed by a Q process. The high level nodes motivating language production (meaning nodes) mobilize high level task nodes and establish goals. Goal setting requires that the brain be capable of predicting how its actions would affect movements produced by others. In other words, the speaker must have a 'theory of mind' [Frith and Frith, 2005].

The high level task nodes interact with the concrete environment and activate nodes representing concrete goals. These are connected to word nodes, which are mobilized. The order in which words and additional morphemes are activated is determined by syntax nodes and the current molding of the neuronal terrain.

Most language use is interactive, combining production and comprehension. Similarly to action observation (AO) vs. execution, comprehension and production use overlapping LSK representations activated during a Q process (see AO below).

Interaction with other people is a fundamental feature of human life. As a result, LS nodes get highly automated, ensuring smooth and rapid production. However, automaticity can be hampered in many ways (stress, speaking in an unautomated foreign language, unclear semantic goals). This can result in temporal pauses, filled using whatever technique the speaker is used to (usually filler sounds, which are more natural than silence due to the mobilization of the sound producing organs).

\footnotetext{
${ }^{62} \mathrm{As}$ in the visual modality.

${ }^{63} \mathrm{LS}$ areas are left lateralized in most people.

${ }^{64}$ The ATL characterization as the hub of linguistically acquired knowledge is a novel contribution of R17.
} 
Report mode. General object, action and valence areas comprise the semantics areas and are bidirectionally connected with LS areas, whose function is to describe object configurations. When language gets highly automated, flow from these semantics areas reaches LS areas and can easily activate LS nodes. Even if external audition is not activated, internal language representations easily are, yielding inner speech (a kind of mental imagery, see below). Thus, the human brain naturally activates language in a mode that continuously reports (shadows) whatever is going on in the brain. In other words, even if thoughts (however defined) can occur without language, it is natural for language to accompany thoughts.

Language does not only shadow the thinking process, it also assists it. By allowing efficient use of a large range of solutions (e.g., because abstract words cover many different situations, and because language allows access to knowledge previously acquired through it), it improves planning and decision making. Thinking, and the tight relationship between language and awareness, are further discussed under internal cognition below.

Evolution and acquisition. This paper does not address language evolution and change. Here, we only note that since there are no particular constraints on LSK representations besides those imposed by the human auditory systems (hearing and articulating), the space of specific possible languages is quite large. As in other forms of biological evolution, people are subjected to environmental and other types of pressures, which change the way they communicate. As a result, different languages can emerge after just a few generations (especially if speakers are not in direct contact with each other). The emergence of language is not limited to large groups of people; even very small groups (couples, families) can evolve their own unique phrases.

Note that since language is implemented by a $\mathrm{Q}$ process, and since $\mathrm{Q}$ processes naturally undergo automaticity, automaticity of communication naturally occurs both between generations and along the life time of a single organism. In the human social sphere, this results in the emergence of customs, rituals etc. In the linguistic domain, it supports the transmission and continued use of language. As in other domains (e.g., vision), there are periods during ontogenic development in which LSK areas exhibit high plasticity, facilitating the acquisition and automaticity of language.

\subsection{Supporting evidence}

The R17 language account is supported by several types of evidence. A brief sketch is given below.

Areas. Language involves the activation of a wide brain network that includes LIFG, perisylvian areas, the ATL, general task areas, the thalamus and the BG [Friederici, 2011]. Sensitivity to sequences and semantics (identified via fMRI and lesions) is mostly identified in BA 45 and 46, respectively. Syntactic sequences (constructions) can be distinguished in fMRI data using BA22 and BA47 [Allen et al., 2012].

Behavior. People can interact with people who do not speak their language well (e.g., tourists), and also with people who do not speak any language well (e.g., very young children), situations characterized by the linguistic notion of 'good enough' processing [Karimi and Ferreira, 2016]. I interpret these obvious facts as indicating that language involves a $\mathrm{Q}$ process. In such situations, the LS part of language knowledge is missing or seriously impaired. Nonetheless, the neuronal gap between the speakers can still be bridged if they are motivated to communicate. In this case, the sensory inputs relevant to the speakers (i.e., the context) helps in triggering the intended meaning nodes. In the case of adult speakers, meaning nodes automatically mobilize LSK nodes supporting their native language (L1). 
The result is a combination of L1 and L2 words, L1 syntax imposed upon L2 words, gestures and sounds. The combination may not conform to any 'valid' linguistic utterance, but it can be used for effective communication if it manages to activate the intended meaning nodes.

During speech, words are generally fused, not separated, and frequent sequences exhibit a general shortening of articulation time. These phenomena are easily explained using the R17 account of the simultaneous activation of different muscles during motor execution (see Q process above).

Speech errors usually involve pieces of prepared speech (words, syllables) articulated in the wrong order. This can be explained by an erroneous transition of the prepared node from prediction to reality mode. Since prepared nodes are active in prediction mode, and since speech transitions are very fast, it is easy for the wrong node to be excited.

Anticipation in language. Anticipations and predictions, on both the LSK and the semantic side, are exhibited in a wide array of experimental language paradigms [DeLong et al., 2014]. Best known is the N400 ERP component, whose amplitude is inversely correlated with the semantic compatibility (degree of anticipation) of a new word. As in motor actions, anticipation can involve a sustained anterior negativity (SAN). [Li et al., 2017b] show that SANs start right after hearing a word that generates relatively focused expectations in a given context (e.g., a verb and its arguments), and increase until the predicted word arrives.

In general, priming phenomena in language are well known, e.g., speakers tend to repeat words and grammatical patterns used recently in a conversation by any of the participants. Priming can be explained via a continued state of excitation of recently active cells, but in some experiments it was shown to result from active mobilization. Using cross-modal lexical priming, [De Goede et al., 2009] showed that the meaning of a verb continues to be active (to prime related words) only as long as its clause is not closed. After the clause ends, all meanings (nouns, verbs, etc) rapidly decay. This provides support for mobilization, goaling and transitions: the meaning of a verb that has already appeared in the sentence continues to prime because one (or more) of the nodes that has activated it still actively excites it. Here, this mobilization is most likely done by syntax nodes, because it stops when the syntactic unit (the clause) terminates.

Syntax is highly automated, using precise predictions that result in rapid non-surprising transitions. There are two early ERP components, the LAN (left anterior negativity) and the ELAN (early left anterior negativity) that reflect short term anticipations. Both are larger in case of structural (syntactic) surprises (e.g., wrong morphological inflections).

Late ERP positivities occur in cases of semantic conflict beyond that of the single word. This can be explained via increased high level competition that involves the activation of a larger number of higher level nodes in reality mode. For example, upon hearing 'The boy kicked...', a prediction for a noun phrase telling us what was kicked is formed, but if the next word is 'by' it generates a surprise that activates new action nodes in reality mode, yielding action node competition. Accordingly, the P600 ERP component is larger when an initial interpretation of the sentence needs to be revised.

Embodied cognition. A large set of experimental results shows that motor neurons controlling various effectors bidirectionally affect language processing in an effector-specific manner [Schomers et al., 2015]. This is easily explained by reciprocal mobilization of motor neurons, task (semantic) and word nodes. For example, when you hear 'kick', the word triggers all action nodes relevant to kick, including nodes implementing your own kicking. This mobilization is usually weak, induced by single features without support from the full context (see also mirror neurons below). Additional related evidence is discussed under 
internal cognition below.

Need-driven acquisition. Oxytocin receptors in left auditory cortex enhance the learning of pup call responses in non-pup-naive female mice [Marlin et al., 2015], providing a link between lateralized audition and motivated conspecific communication.

\section{Internal Cognition}

Internal cognition refers to explicit awareness of external stimuli and internal states, and to functional capacities that do not involve movement, such as imagination and thought. These are fundamental to the human experience and must be addressed by any brain theory. Here we present a mechanistic account of these phenomena. Our account of imagery is completely novel, while our accounts of awareness and emotions share some aspects with existing accounts and are novel in their integration with the $\mathrm{N}$ and $\mathrm{Q}$ processes.

\subsection{Awareness, consciousness}

Humans have a subjective feeling of consciousness, a sense of awareness of external events and internal states and thoughts ${ }^{65}$. Although subjective feelings are (at present) not directly accessible to science, consciousness can and should be investigated as a biological phenomenon [Koch, 2004]. The major approach to address awareness in a scientific way is via reportability [Dehaene, 2014]. Humans can report feelings by using language. There are several questions to answer. First, what mechanisms support such reporting? Second, can consciousness exist without reporting? in other words, does it arise when the reported event occurs, or only during (and thus due to) reporting? Third, does consciousness exist without language? Finally, are there brain areas that are the 'seat' of consciousness?

All actions involve action nodes active in response mode and object nodes activated in internal mode serving as action goals. Execution continues until the goal nodes switch to reality mode. Let us first examine awareness of some external sensory event. Suppose that you are asked 'do you see a ball?'. Language nodes translate the question to action nodes implementing a visual search task whose goal is a ball. When the search is successfully met, the goal nodes switch to reality mode and trigger a transition to the next action in the sequence, which in this case is the language action reporting 'yes'.

In light of this, it seems that all that is needed for reporting is something (language here) to establish the correct action sequence (search) quax (and motivation to drive it). This holds also for reporting awareness of things that are no longer present in the sensory input (e.g., our actions after executing them). In this case, the search quax is completely internal. We can report such things because the completed action's goal configuration is still partially excited, so it immediately meets the search quax established by language. When the completed action has been executed in automatic mode, it cannot always be reported, because automated actions leave very weak traces in the brain.

This account answers the first question above (reporting mechanisms) for external events, but it does not address the unique feeling of consciousness that people claim to have. As others (e.g., [Craig, 2014]), our view is that this feeling arises from body states. The insula and $\mathrm{mPFC}$ receive interoceptive sensory inputs conveying the state of internal body organs and tissues, and these can be reported in an identical manner to that described above for exteroception. Note that the insula is adjacent to the Sylvian fissure, the main LS

\footnotetext{
${ }^{65}$ Consciousness can be viewed as awareness of internal states. In order not to get into terminological arguments, we use these two words synonymously.
} 
language area. In fact, it is closer to language areas than to all other cortical areas, essentially being the medial language area. Even the simplest linguistic utterance (e.g., pointing to an apple and saying 'apple') involves reporting an awareness of an external object. Thus, there is an intimate connection between language, awareness and interoception ${ }^{66}$.

It can be argued that some representation of 'self' is an essential part of an awareness report. That is, for external awareness the reporter should be capable of seeing her/himself as participating in the situation, and internal awareness is equivalent to the report that the self exists. Self is probably a high level node that generalizes many external and internal sensory representations, and may hence be most strongly represented around the anterior insula. Note that language facilitates the acquisition of the concept of self, but there is no theoretical reason why it cannot be acquired without language. All that is required is a sufficiently large UQS in interoceptive areas.

For the second question, some kind of consciousness may exist without reporting, but human brains appear to be incapable of showing it or imagining it. It can be argued that all attended execution possesses inherent consciousness, because it can in principle be reported later given reporting capacities. However, even so, the only thing that is special about awareness and that allows distinguishing it from non-awareness is reporting.

For the third question, the role of language is to establish a search quax and its final reporting action. It is certainly possible for search quacia to be established for other reasons and by language-less animals. For example, a sensory cue (e.g., sound, smell) can activate (in internal mode) nodes representing objects having a strong positive valence (e.g., an offspring), and this activation can in turn excite the interoceptive nodes associated with this object. These internal mode activations can drive the formation of a search quax. Upon successful detection of the object, the animal can execute a variety of actions that can be viewed as reports. This moment can be argued to be one in which the animal has awareness of the object. Conversely, consider an animal that emits auditory signals when its offspring is separated from it. These are innate distress signals triggered by an unsuccessful search, and can thus be considered an awareness report.

Regarding the last question, the insula (and possibly mPFC) is essential for internal awareness, and all brain areas that are essential for quax formation and execution are essential for both kinds of awareness. Specifically, the thalamus is viewed by many as being especially essential for awareness [Ward, 2011]. Our analysis implies that this is probably true, simply because the execution of adaptive quacia requires the thalamus.

There is a well known (and disputed) distinction between the 'easy' problems of consciousness (approximately those discussed above) and the 'hard' problem of consciousness, which asks why we experience some sensory inputs as having their own unique 'qualia' (for example, why 'redness' evokes a different experience from 'blueness'). My answer is that we sense things as different exactly when they yield different brain responses, and when we report this fact to ourselves. Any object node can potentially be sensed as different from all others. This solves the hard problem.

The account of awareness in this section enjoys the advantage that it relies on brain capacities that are known to exist. It is of course possible that awareness involves a unique type of process, not the $\mathrm{Q}$ process, but an account that uses known processes is more parsimonious than an account that posits a new unknown process type.

\footnotetext{
${ }^{66}$ Recall that interoceptive inputs to the insula are a primate evolutionary innovation. This may have been a prerequisite for the emergence of language.
} 


\subsection{Emotions}

The word emotions refers to a set of words that includes happiness, surprise, fear, anger, disappointment, sadness, disgust, etc. Each of these words is an abstract word, i.e., a word describing a variety of specific concrete situations, sometimes quite different from each other. The main questions regarding emotions are what brain mechanisms they involve and whether they exist without words. These are exactly the questions we discussed under awareness above, and our answers are similar. As in internal awareness, emotions report the activation status of interoceptive nodes. What is unique about emotions is that emotion-carrying situations strongly correspond to $\mathrm{N}$ modes ${ }^{67}$. For example, 'happiness' corresponds to the satisfaction $\mathrm{N}$ mode, 'euphoria' to the consume $\mathrm{N}$ mode ${ }^{68}$, and 'fear' to the alert $\mathrm{N}$ mode.

The association of emotions with interoception is similar to existing well known views equating emotions with bodily states [Damasio and Carvalho, 2013]. The R17 account improves upon this observation by using the mechanistic account of awareness via reporting described above and by associating emotions with $\mathrm{N}$ modes. Note that although $\mathrm{N}$ modes are strongly related to innate responses, innate responses cannot be equated with emotions. Emotions are reports, not responses. Hence, most experimental paradigms involving non-human animals do not investigate emotions. For example, 'fear' is an emotion, but so-called fear research actually deals with threat responses [LeDoux, 2015].

There are more emotion words than $\mathrm{N}$ modes, due to two reasons. First, many emotions refer to sub-N modes distinguished according to participants (e.g., people-specific vs. general). Second, since the timing of the reporting action is decoupled from event execution, the reported situations can be distinguished by their timing relative to the event (before, during or after it), and by reanalysis of event outcome.

As a detailed example, consider the emotions associated with the failure $\mathrm{N}$ mode, which is characterized by freezing and by not being able to come up with an action except disengage, and is promoted by the Rgen DYN. Frustration and despair are pre-failure situations involving effortful execution that induces DYN release. In frustration the result is still not clear, while in despair failure is imminent (and the goal is commonly more important). Insult, embarrassment and shame report quick failures involving other people. Insult reports losing a type of social fight. Embarrassment and shame report specific freeze-like social responses ('I want them to stop seeing me', the original role of freezing). Hurt is like insult but commonly comes after a social approach action rather than after a fight-like event. Longing is a social failure stemming from separation from a known person (or sometimes an object). Loneliness is similar but without a concrete target. Sadness and depression describe general failure situations, depression being a deep, prolonged sadness. Disappointment refers to approach actions whose goals were not attained. Regret and guilt stem from post-hoc analysis ('I should have done something else'). In regret, the event itself has usually terminated in failure, while in guilt it has usually terminated in success. Both constitute post-hoc failure in that you want to 'disengage' from the act.

Viewing emotions as reported $\mathrm{N}$ modes is strongly supported by familiarity paradigms, see below. In addition, increased interoceptive vagal inputs are associated with higher frequency heart rate variability (HRV), a measure of beat-to-beat temporal heart rate changes, which correlates with emotion recognition [Quintana et al., 2012]. Additional support for the relationship between interoception and innates (and thus $\mathrm{N}$ modes) and emotions is

\footnotetext{
${ }^{67}$ Technically, this holds for all feelings too, so feelings and emotions are synonymous. In everyday use, the term 'emotions' is used for situations involving stronger flow and having longer term effects.

${ }^{68}$ Indeed, euphoria is commonly used to describe the feeling induced by heroin, whose main effect is achieved via MORs, the receptors for the consume Rgen BEND.
} 
given in [Craig, 2014, Damasio and Carvalho, 2013, LeDoux, 2015].

\subsection{Familiarity \& recollection}

An important type of feeling is the feeling of familiarity (FOF), reported by 'I have met this object before'. When the object is a piece of knowledge, FOF is sometimes called feeling of knowing (FOK). Familiarity can be contrasted with another type of recognition, recollection, where we report specific information (name, past events, general knowledge) related to the object.

Recall that objects generate two input flow channels, into cortical object areas and into the amygdala. CeA generates innate (including autonomic) responses, and BLA passes object-innate associations into cortical valence areas. The executed innate responses yield changes to bodily state, which are also reported to valence areas, as interoceptive inputs.

When an object is encountered for the first time, there are no existing associations. If the encounter involves a memory-promoting response (i.e., an acute or interaction $\mathrm{N}$ mode), learning processes induce object and valence representations. During subsequent encounters with the object, its object and valence representations are activated. The object nodes are connected with other object and context nodes, and together they support recollection. Indeed, recollection is strongly associated with the hippocampus, which represents arbitrary inter-object and object-place associations.

When asked whether an object is familiar, a person can rely on successful recollection in order to answer 'yes'. However, it is not rare for an object to induce familiarity without recollection ('I know this person but I can't remember where we met'). Our view is that this feeling relies on the object's interoceptive valence nodes. These are sensory nodes and can thus serve as goals for action nodes. When asked if you are familiar with an object, language nodes establish a search action plan that uses these valence nodes as goals. If they are excited, this allows a 'yes' report to be made ${ }^{69}$.

Familiarity can induce both increased and decreased alerts. Familiar objects can induce higher alerts than unfamiliar ones because everything else being equal, familiar objects generate stronger responses due to their existing object and valence representations. For example, when we encounter a familiar person in an unexpected context we experience an alert response that does not occur for unfamiliar people (the 'butcher on the bus' phenomenon). In other words, familiar objects are more salient than unfamiliar ones and trigger the orientation of attention. Conversely, if a person or animal makes an alerting surprising appearance in a given situation, the alert may be suppressed when they are recognized as familiar and non-threatening. This is related to the specific valence of the object, not to the fact that a stored familiar valence exists.

There is substantial experimental support for the involvement of interoception in familiarity, especially from the reaction to faces. The measured sympathetic responses are mainly sweat gland activity known as electrodermal activity (EDA) (also skin conductance response (SCR)), and pupil dilation. For example, [Bonifacci et al., 2015] found a significant EDA increase for personally familiar faces compared to unfamiliar ones. Moreover, the response for relatives disappeared faster than that for close friends. In [Kafkas and Montaldi, 2015], familiar objects induced larger pupil dilations than novel ones, with larger dilations for surprising objects in both categories (as expected). FOK involves the anterior insula, ACC, PCC [Kikyo et al., 2002], and mPFC [Modirrousta and Fellows, 2008], all valence areas.

\footnotetext{
${ }^{69}$ The positive 'I found it!' feeling at the end of successful effortful recollection is different, stemming from the success $\mathrm{N}$ mode associated with the search process, not from the valence of the recalled information.
} 
Recollection and familiarity dissociate. In 'tip of the tongue' (TOT) and related states, people do not manage to recall a word even though they feel that they know it. People with prosopagnosia (face recognition impairment) show the normal EDA distinction between familiar and novel faces, without recollection. Conversely, patients with bilateral ventromedial frontal damage can recognize familiar faces without showing this distinction [Tranel et al., 1995].

People who recall an object can rely on this fact to say that they are familiar with it as a response strategy, even if their autonomic responses to it are impaired. In the rare Capgras syndrome, people are incapable of applying this strategy to people (or less frequently, to objects) possessing a great emotional significance, presumably because in this case the autonomic response is essential for full recognition. Patients can correctly identify a close family member, but report that they feel that $\mathrm{s} / \mathrm{he}$ is an impostor. Capgras is associated with mPFC damage [Fiacconi et al., 2014], and patients do not have the EDA familiar-novel distinction to faces [Brighetti et al., 2007].

Familiarity judgements for objects that are not strongly need-related and/or that have been encountered very recently in a spatial context may rely on the perirhinal, entorhinal and parahippocampal cortices at the medial temporal lobe, which are key areas in the representation of objects in context. Thus, EC lesions can impair familiarity judgements in study list items while recollection remains intact [Brandt et al., 2016]. The hippocampus is essential for novel object-context-valence combinations and is thus associated with recollection.

\subsection{Imagery, thinking}

Humans have a capacity for sensing objects that are not supported by external sensory inputs, in a way that involves a reportably different experience from how reality is sensed. Such imagery involves the visual and auditory modalities, and can be used to access several content types, including objects, episodic memories, words and sounds. Temporally changing (dynamic) imagery includes visual motion (where motor imagery includes movements in peri-personal space), music, and inner speech. In addition, vision also allows static imagery for most people. We use the terms imagery, thinking and imagining synonymously. Like all brain processes, imagery can be need-driven (planning) or automatic (e.g., a song playing in one's head without control). In all forms of imagery, the object lacks details and vividness. Visual imagery includes high level object contours without color, form or textural details, and auditory imagery does not include rich timbre.

The main questions about imagery are what its evolutionary role is, how it is mechanistically implemented in the brain, and whether non-human animals are capable of it. Here we provide novel answers to these questions.

Evolutionary role. Evolution is guided by the degree by which organisms answer their needs. Need satisfaction is managed by the $\mathrm{N}$ process, so the evolutionary role of a brain capacity should be explained by showing how it integrates with the $\mathrm{N}$ process. Imagery is clearly not an innate response, so non-automated imagery is part of the acute mode. More specifically, it commonly occurs after alerts and before focused execution, when flexible (novel) but usually not urgent responses need to be found. In other words, imagery (planning) is the R process non-urgent DM mode. Organisms with better response planning capacity have a clear evolutionary advantage.

Since planning is done prior to movement, and since part of the brain's initial adaptive response to surprise is to stop on-going execution, imagery should be intimately related to movement suppression. Indeed, phasic release of SER, the Rgen promoting planning, 
suppresses responses (see above).

Mechanism. Since the Q process underlies the generation of external movements, and since the brain's architecture is tailored for running it, our view is that a $\mathrm{Q}$ process underlies imagery as well. Imagery is a $Q$ process that does not include low level sensory nodes active in reality mode. This occurs because such nodes are not excited by sensory input, which is normally essential for their activation in reality mode.

There are several issues that should be addressed with respect to this account. First, the external Q process is powered by BU flow generated by sensory inputs. What drives imagery, which lacks these inputs? Second, which object nodes participate in imagery and in which mode are they active? Third, what is the role of motor nodes in imagery? Fourth, transitions are normally induced by sensory inputs. What induces transitions in imagery? Fifth, which areas are involved in imagery? Are there areas dedicated only to imagery? Finally, the brain can distinguish between imagination and reality and report the difference. How is this type of awareness implemented?

Drive. Internal needs excite neurons in motivation areas via OX (see above), providing continuous frontal drive in various networks. The flow networks are strongly interconnected all over cortex, short distance connections being stronger than long distance ones. Thus, there is no reason why frontal drive would not be able to activate frontal neurons in other nodes (even response neurons if they are not very large).

We note that an important trend in brain evolution apparent in recent ancestors of humans is a significant size increase, especially in PFC, PPC, lateral temporal cortex, and the insula, including denser connectivity of prefrontal pyramidal neurons [Kaas, 2013]. The greatest difference between humans and other ape species is in PFC white matter (myelinated axons supporting strong flow) [Schoenemann et al., 2005]. These data imply that the capacity of frontal neurons to converge upon less frontal neurons and excite them effectively has greatly increased in animals having higher internal cognition capacities such as planning and tool use.

The moment at which non-urgent DM (i.e., planning) terminates is called decision time (DT). It is the equivalent of Q process competition resolution. DT arrives when a satisfactory plan has emerged, which occurs when the plan reaches an end-state goal having an appropriate valence. Amygdala BLA-cortex projections reach the DM network, providing valence-loaded BU drive to the planning Q process. During planning, the goal is activated in imagery mode (below), and its valence is accessible by cortical connections with BLA. The actual decision to move should be mediated by mPFC, the main motivation area. Indeed, the anterior midcingulate cortex (aMCC), which is strongly connected with all motor regions, is critical in volitional motor control [Hoffstaedter et al., 2014].

Object nodes. Low level sensory areas are identified in fMRI as active during imagery. For example, visual imagery extends as low as V1 [Pearson et al., 2015]. This is easily explained via internal network activity, since internal (mostly DM) neurons are mobilized by quax neurons even if they do not join the quax. Sensory inputs are only essential for activating low level nodes (object and action) in response mode, due to the relatively large size of their response neurons.

The main question related to object nodes is which nodes represent the imagined objects. There are two possible accounts. First, they could be located in high level object areas or even frontal action areas (recall that the representation of categories could be frontal). In this case, the fact that their activation is not perceived as occurring in reality would be due 
to their high level location, even if they are activated in reality mode ${ }^{70}$. Alternatively, they could be located anywhere but activated in internal (most likely DM) mode.

In the first option, imagery is implemented by an ordinary Q process, and the only difference between imagery and movement quacia is that the former do not include low level object nodes in reality mode. In the second option, imagery is implemented by a $\mathrm{Q}$ process that is different from the external one in two ways. First, external Q transitions are triggered by the activation of nodes in reality mode, while here they would be triggered by the activation of internal network neurons. Second, external quacia do not include focused DM paths without reality network activity (DM paths must be focused during imagery, because we imagine specific objects). We discuss this issue with transitions below.

Motor nodes. There is incontrovertible imaging evidence that areas activated during motor execution are also activated during imagery, in a limb-specific manner [Guillot et al., 2014]. Moreover, execution and imagery exhibit similar ERPs [Machado et al., 2013]. As for low level object nodes, these data can be explained as DM network activity coupled with a lack of activation of the response network ${ }^{71}$. However, there is also evidence for response network activation during imagery, since imagery involves increased corticospinal excitability (albeit weaker than during execution) [Grosprêtre et al., 2016]. In all motor areas, including high level ones, response neurons project to the spinal cord. Why would response neurons be activated during imagery? and if so, why does movement not occur?

Our answer is that spinal excitability during imagery is due to gMTNs, which do not induce movement. A simple explanation of why gMTNs are active during imagery is that the DM mode primes execution circuits during movement planning to facilitate the switch from DM to execution. Thus, gMTNs can be viewed as an innate part of the prediction network $^{72}$.

There is good (albeit indirect) evidence supporting this account. Differences between the stretch and $\mathrm{H}$ reflexes in imagery suggest that it involves increased gMTN activity [Aoyama and Kaneko, 2011, Jeannerod, 1995]. Mental computation yields increased excitability of spinal reflexes due to gMTNs [Rossi-Durand, 2002]. In addition, imagery involves a decrease in the steady state motor EEG beta rhythm, which has been explained via cortico-spinal-cortical loops involving proprioception [Aumann and Prut, 2015]. Such a reduction can be due to increased gMTN excitability (as in alpha oscillation decreases during increased attention, see below). Finally, there is substantial evidence for the involvement of the motor system in cognition (see embodied cognition under language above), a lot of this evidence involving imagery. For example, there is increased excitability of lip muscles when reading the letter ' $\mathrm{P}$ ' (whose articulation involves the lips), but not the letter ' $\mathrm{T}$ ' (whose articulation does not involve the lips), with opposite results for tongue muscles [McGuigan and Dollins, 1989]. Similarly, forced relaxation of facial muscles (lip, forehead) reduces verbal rumination [Nalborczyk et al., 2017].

A simple explanation of why movements are not produced during imagery is that low level motor nodes are still not active in reality mode. Movement is initiated at decision time, when M1 and PMC accumulate enough excitation due to motivation and valence flow reaching $\mathrm{mPFC}$ (in particular aMCC).

Transitions and node activation mode. In movement execution, goal attainment (the

\footnotetext{
${ }^{70}$ The reason why it is harder to imagine touch than images and sounds may be because sensory touch representations (in S1, S2) are close to the nodes representing their responses (in M1), which does not leave enough cortical space for the formation of high level nodes.

${ }^{71}$ It could be proposed that there are motor nodes dedicated to imagery. However, we can imagine everything that we execute, and it is not reasonable that the brain has two copies of each motor node.

${ }^{72}$ If they are solely activated by adaptive nodes, they cannot be viewed as an innate prediction network.
} 
selection of object nodes) and transitions (the selection of the next action by attained goals) are done by sensory inputs. In imagery, object nodes are selected by flow generated by other object nodes serving as cues, in addition to flow generated by frontal task nodes. For example, when you try to find an animal whose name starts with a particular letter, the letter and the word 'animal' serve as cues that generate flow that triangulates frontal flow to activate the correct nodes.

The nodes representing the cues are also activated in imagery mode. Since the flow networks are internally connected, cue nodes can generate flow to activate other nodes in imagery mode regardless of whether imagery involves the internal networks or the response network. Thus, there is no inherent constraint that prevents transitions from being triggered by the activation of a node in focused DM or prediction mode.

Note that the BG and thalamus do not provide such a constraint. First, the prediction network projects to the STR, and even the DM network may do so. More importantly, the $\mathrm{BG}$ are required for adaptive quacia in order to allow cortex to recruit the thalamus, which is mainly needed in order to sustain response network execution. If imagery does not require the response network (for the imagined objects), the thalamus may not be needed as well (the thalamus may be needed to sustain internal network activity, an argument answered by the first point).

Some nodes must be active in reality mode during non-automatic imagery, in particular motivation nodes, high level task nodes, and the nodes driving gMTNs (these are driven through corticospinal projections, so their activation requires the response network). Moreover, there is clear evidence that motor imagery can involve the BG [Hétu et al., 2013]. SER, the Rgen promoting DM, has both suppressive and excitatory effects in the BG. Thus, the role of the latter may be to support imagery.

Areas. Since imagery is TD driven, it always involves frontal areas. The other areas involved depend on the type of the imagined content. Episodic memory involves the extended hippocampal system (see above) [Schacter et al., 2017], music involves the auditory cortex, motor imagery involves motor areas, and imagining needs involves valence need areas (mPFC, the insula) [Lin et al., 2015].

Relatedly, there is a network of brain regions consistently activated during relaxed internal cognition, known as the default mode network (DMN) [Raichle, 2015], whose core areas are mPFC, posterior cingulate cortex (PCC), lateral and medial temporal cortex, and the posterior inferior parietal lobule. Thus, the DMN largely overlaps with the episodic memory network. In a study using autobiographical and visuospatial planning tasks, the former engaged the DMN, while the latter engaged the dorsal attention network (dlPFC, FEF, visual motion area MT+, motor areas, and the superior parietal lobule) [Spreng et al., 2010]. Both tasks also engaged a general frontoparietal control network located between these two networks.

Being a DM and thus a longer term response, imagery should be supported by action areas that are more anterior than the areas supporting immediate movement. The leading candidate for this role is the supplementary motor area (SMA), especially its preSMA part. Indeed, these areas are strongly associated with imagery, including auditory imagery [Lima et al., 2016] and semantic memory access [Hart et al., 2013]. SMA neurons project directly to the spinal cord, and their projections and the M1 ones converge on the same motoneurons [Maier et al., 2002]. The spinal effect of SMA is weaker than that of M1, has a different nature, and is related to silent periods more than to movement [Kikuchi et al., 2012]. The SMA contributes to the early readiness potential shown in voluntary movement (i.e., to movement planning) [Jahanshahi et al., 1995]. SMA also shows stronger activation in imagery than in hallucinations [Raij and Riekki, 2012], which are a kind of imagery 
that stems from brain impairment rather than from the $\mathrm{N}$ process. In this view, gMTNs are driven by spinal projections of the SMA and preSMA response network.

Supporting our predicted connection between imagery and movement suppression, there is evidence that preSMA mediates suppression of the losing alternative during strong competition [Duque et al., 2013]. Surprises (including errors) induce global stopping via the right inferior frontal cortex (RIFC) and preSMA, while the anterior insula is involved is less immediate stopping [Wessel and Aron, 2017].

Note that many of the areas involved in imagery are medial areas (the hippocampal system, mPFC, preSMA, SMA, the insula), which accords with imagery being a needdriven DM process (recall that valence areas are generally medial).

Reporting. When a person is asked whether a recent experience involved reality or imagination, a search quax is established as in all reporting questions. To be able to answer, the quax needs to include goal nodes that are active only in case of reality or only active in case of imagination. As noted above, the insula contains the former, and preSMA may contain the latter. The insula seems a more probable candidate, because preSMA may participate only in motor imagery and because the insula supports other awareness questions.

Language LS areas continuously generate reports of ongoing actions, and most of these reports are silent. It would be efficient if these areas could mediate speech suppression. Supporting this, the right IFC is strongly associated with stopping motor actions (as noted above), so the left IFC (the language syntax area) may be able to do this too, through a sub-area specializing in suppressing speech.

Non-human animals. Imagery is an R process DM mode, and the brains of all animals are managed by an $\mathrm{R}$ process that includes DM. Hence, animals can be argued to be capable of thinking, unless thinking is specifically defined as imagery that uses language. There is evidence that animals imagine action scenarios in the CA1 field of the hippocampus before executing them [Pfeiffer and Foster, 2013]. More generally, sharp wave ripples (SWRs) originating at the hippocampus and present in many brain areas occur during immobile wakefulness and sleep [Buzsáki, 2015], are essential for memory formation, and are involved in navigation planning [Roumis and Frank, 2015]. SWRs involve rapid transitions between negativities and positivities and thus provide support for a Q process-like execution during internal cognition. SWRs are most prominent at event and frontal areas and least prominent in sensory areas, supporting their interpretation as planning.

\subsection{True \& false recollection}

Both recollection of real events and imagining invented events involve imagery of events that are not taking place in immediate reality. How does the brain distinguish between these situations? A natural answer is that the former involves familiarity nodes, while the latter does not. If so, how does the brain distinguish between real past events and imagined events that have already been imagined in the past? both should possess familiarity signals.

A possible answer is that the latter situation involves recollection of the fact that the event has been imagined. This could be only a small part of the neural activation involved in recollection, and people indeed frequently fail to make the distinction correctly. For example, it is not rare for people to erroneously remembering being present in some event. This false recollection usually happens when people are familiar with the real event (having heard of it or seen it on video) and see themselves as natural participants in it. This happens because recollection works via arbitrary combinations of different nodes, making it relatively easy for incorrect nodes to get included in the quax. In this case, recollection 
is accompanied by familiarity nodes, making the person sure that the false recollection is correct.

Note that repeated recollection of an event can modify its brain representation, since all non-automated quacia induce plasticity changes. This phenomenon is called memory reconsolidation [Alberini and LeDoux, 2013]. In particular, repeated recollection increases the probability that event details would get distorted, because any content node that has accidentally been incorporated into the imagination quax is now associated with a familiarity signal. This explains the well known problems with eye witness accounts.

\subsection{Summary}

Internal cognition has two main orthogonal axes, emotions and imagery. Emotions, internal awareness (consciousness), and the feeling of familiarity arise from reports of interoceptive nodes reflecting $\mathrm{N}$ modes. The insula (interoceptive cortex) is thus a core internal cognition area. Imagery is the $\mathrm{N}$ process non-urgent $\mathrm{DM}$ mode and is used for planning responses before their execution. It is implemented by a $\mathrm{Q}$ process that involves action nodes and high level object (or category) nodes activated via cues to induce transitions. The special quality of imagination stems either from the non-activation of low level sensory nodes or from object node activation in internal mode. Motor circuits are primed during imagery by SMA/preSMA nodes via gMTNs, which can thus be viewed as an innate DM/prediction network. Imagery and external execution are supported by different familiarity signals and different areas (with overlap in motivation and task areas), allowing the brain to report the difference between them. This includes the distinction between re-imagery and recollection. Thinking, planning, imagery, suppression of losing alternatives in action selection, error responses, and stopping an on-going action are all related to the $\mathrm{N}$ process DM mode, and their respective areas exhibit an anterior to posterior gradient.

\section{Additional Evidence}

The amount of evidence supporting various aspects of R17 is huge, and a single paper can only present a small part of it. In this section I present several additional important results and paradigms that support the theory. Brain disorders also provide substantial supporting evidence, but doing any justice to this topic would require too much space so it is presented in other papers.

\subsection{Action observation, mirror neurons}

Action observation (AO) is similar to imagery in that it is a $\mathrm{Q}$ process without self movement. Indeed, like imagery, AO shows a bilateral reduction in beta band LFP [Alegre et al., 2010]. AO is different from imagery in that many transitions are triggered by external movement. The progression of events is externally guided, but the process may involve thinking episodes between or even in parallel to external transitions.

As normal in the $\mathrm{Q}$ process, the frontal nodes triggered by the observed actions actively mobilize other nodes, which is most strongly manifested in the DM network. The famous mirror neurons [Rizzolatti and Sinigaglia, 2008] are such mobilized neurons. Mirror neurons fire both when an animal performs an action and when it observes a similar action. The fact that the actions are similar means that there are many features (sensory or higher level) common to the two situations, so it is natural that there should be many neurons mobilized in both situations. 
There is solid support for mirror neurons being part of the DM response. Mirror neurons can predictively activate before an action [Kilner et al., 2004], including before withheld actions [Maranesi et al., 2014]. They are easily found in brain recordings, are located in $\mathrm{L} 2 / 3$, and are found in many brain areas. Because mirror neuron activation is triggered by features (i.e., not by complete objects or situations but only by some of their aspects), it does not require that the animal be capable of performing the same action itself.

Language comprehension is the language analogue of action observation. Indeed, there is substantial evidence that production and comprehension share syntax areas [Segaert et al., 2012] and predictive mechanisms [Pickering and Garrod, 2013].

\subsection{Place \& grid cells, theta phase precession}

In addition to its role in episodic memory, the hippocampus is famous for containing place cells [Buzsáki and Moser, 2013], which are active when the animal reaches specific locations and are thus thought to be part of the animal's spatial map representation. Place cells form in locations of meaningful events, and take time to emerge in new environments. Thus, they constitute the prototypical content type represented at the hippocampus, spatial events, combining place, object and valence. Other types of space-related cells are MEC grid (below), head direction, border and speed cells.

The hippocampus shows a dominant EEG theta rhythm (4-10Hz) during rodent activity. Under certain conditions (especially uniform running) place cell firing exhibits theta phase precession, i.e., the cell fires once in every cycle of the theta rhythm, firing earlier in the cycle in consecutive cycles [Malhotra et al., 2012]. Many accounts have been proposed to explain theta and theta phase precession. Here we describe a novel account for these phenomena, in which place cells are activated as goals of locomotion actions.

The theta rhythm. Attaining a goal involves suppressing the TD networks and activating the response network, so it should be reflected as a relatively strong positivity in the EEG. This is the theta peak. If running is done at a constant speed, the environment is known, and setting location goals is necessary for smooth running, then place cells should be approximately evenly spaced, which is indeed the case [Cei et al., 2014]. This is why EEG peaks are equidistant. In other words, this is why hippocampal theta oscillations occur.

Phase precession. When the animal reaches the place serving as an action goal, the action completes. As is usual during the $\mathrm{Q}$ process, an attained goal triggers the next action, which innervates a place cell as its goal. Hence, a new place cell activates when an existing one stops. In other words, the number of active place cells during locomotion is constant. Since the animal runs at a constant speed, and since locomotion is highly automated, we can assume that the charging rate of place cells remains the same throughout execution. This means that the cell should fire a few times and stop, which is exactly the behavior shown by place cells. Now, the theta peak corresponds to attaining a goal (see above). It is clearly faster to activate an action's goal (place cell) than to attain the next goal. Thus, a newly opened goal is specified at or right after the peak of the theta cycle, and it fires somewhere inside the theta cycle (before the next peak). Because the time to fully charge it to activation is smaller than the theta cycle and its charging continues at a uniform rate, its next firing would be earlier in the next theta cycle. This goes on until the animal attains the location goal, when the cell's firing coincides with the theta peak. This explains theta

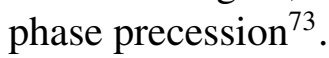

\footnotetext{
${ }^{73}$ Supporting this account is the fact that phase precession occurs also in the STR and other locations [Malhotra et al., 2012], as it should, because all executing adaptive nodes drive flow through the STR.
} 
Grid cells. MEC cells active when the animal reaches locations arranged in a triangular grid pattern are called grid cells [Moser et al., 2014]. MEC neurons exhibit a topographic change in grid size along the dorso-ventral (i.e., the space-valence) axis. Grid cells are tightly connected to place cells (via anatomy and phase precession), so we can assume that they are related to locomotion too. They phase precess, so they are action goals. Grid patterns are independent of particular environments, which points to goals expressed in an egocentric reference frame. This is supported by grid cells being essential for navigation only without landmarks, e.g., in the dark [Poucet et al., 2014], and by pattern loss at the boundaries of the environment, where locomotion manner dramatically changes. The grid cell pattern regularity also accords with the regular rhythm of locomotion movements. We conclude that grid locations express something like 'this is where I get if I move in this direction at this velocity'. Grid cells represent goals of automated, event-independent locomotion actions.

\subsection{Conditioning, generalization \& extinction}

Conditioning is the valence learning scenario in which a neutral object (conditioned stimulus $(C S)$ ) is being associated with a simultaneous (or slightly preceding or following) nonneutral innate action triggered by an unconditioned stimulus (US). Conditioning usually results in the execution of innate responses upon CS occurrence, the capacity we named innate+. However, since CSs are learned, cortex can exert control over this execution to restrain it. Here we describe a detailed novel account for how this is implemented.

In tone-induced fear conditioning, the animal hears a tone followed by pain (e.g., foot shock), which elicits a freeze response (possibly following a brief 'flight'). After training, when the animal hears the tone alone, this strongly excites amygdalar neurons involved in the pain quax. A strong association of active low level sensory features (the tone) with innate pain response paths is formed in BLA and $\mathrm{CeA}$, where CeM triggers a freeze response through PAG projections and issues an alert via the LC and the HT. Similarly, a triggering of an innate response by exposure to a specific spatial context (contextual conditioning) can occur following the formation of an event representation at the hippocampus. An association with an approach (positive) innate can be created in the same manner, as in Pavlov's dog experiments pairing food and sound, and in the conditioned place preference (CPP) experimental paradigm.

Sensory and innate amygdala inputs meet at CeL. There is some evidence that they reach two distinct neuronal populations. About half of the CeL neurons express protein kinase $\mathrm{C}$ delta (PKCD), and form reciprocal GABAergic synapses with non-PKCD neurons [Haubensak et al., 2010]. The PKCDs receive glut input from parabrachial nucleus (PBN) CGRP expressing neurons (but also from some PBN non-CGRPs) [Cai et al., 2014], and their activation/silencing suppresses/increases eating. Similarly, the PBN CGRPs convey a gastrointestinal signal required to establish taste aversion [Carter et al., 2015], convey pain to CeL CGRPs (many of which express PKCD), and are essential for pain response and memory [Han et al., 2015]. CeL PKCDs convey US flow to LA, and many of them reduce activity with learning [Yu et al., 2017]. When the CS appears after conditioning, the PKCDs are silent and a non-PKCD population is active, yielding freezing [Ciocchi et al., 2010, Haubensak et al., 2010].

In light of these data, we propose that CeL PKCD and non-PKCD neurons are activated during innate and adaptive (innate+) responses, respectively. Activity of the PKCD isoform is independent of $\mathrm{Ca} 2+$, conforming to innate (vs. learned) execution. In addition, the PKCDs do not express CRH/DYN, which mark adaptive responses. During condi- 
tioning, the PKCDs, which receive innate BU flow, recruit (coordinate with) non-PKCD neurons excited by the sensory flow from the CS. After conditioning, the CS activates only the non-PKCDs, because there is no innate BU input. The cortical area handling innate BU flow (PKCD alerts) is the infralimbic (IL) mPFC, while the adjacent prelimbic (PL) $\mathrm{mPFC}$ is involved in adaptive (non-PKCD) responses ${ }^{74}$. IL mPFC projections to ICMs is to MOR-poor areas [Pinard et al., 2012], supporting its role as mediating non-approach innates (e.g., freeze) rather than consume. The PKCDs also support positive and locomotion innates, since many of them express OT and ENK [Haubensak et al., 2010, Day et al., 1999].

An independent issue is how a transient CS whose appearance precedes that of the US (e.g., the sequence tone (CS), empty delay period, US) is associated with the innate, as in trace fear conditioning paradigms. A possible answer is that learning of acute events is assisted by various agents (NE, DA, ACh, GCs), and these operate on all neurons that are excited, including those that have been activated a short time before the acute event.

In summary, CeL contains a PKCD population conveying innate sensory inputs and a PKCD- population trained by the PKCDs to support innate+ (conditioned) responses. Both populations suppress CeM neurons and thereby disinhibit innate responses such as freezing. PFC can utilize the PKCD- neurons to execute some innate responses in adaptive mode. In addition, PFC can excite the ICM that suppresses BLA, which allows innate responses such as consume (since the ICM expresses MORs). PFC can also prevent innate responses by exciting BLA, which excites CeM and competes with disinhibiting inputs.

Generalization. Following conditioning, when the CS is activated by sensory input, BLA communicates its valence to cortical valence areas. The activation of the CS is usually accompanied by other sensory inputs, and these are included in the resulting quax as well. If the CS, its valence, and such inputs are active together a sufficiently large number of times, these new sensory inputs can acquire the valence of the CS and generate innate responses on their own (i.e., become a new CS, without the first one). This conditioning process is called generalization. Generalization is sometimes measured through the ability to discriminate between the CS and different sensory input that resembles it (CS-, e.g., a different tone). Resemblance means that there is quax overlap when they appear, making it easy for the CS- stimulus to induce a quax that includes the innate response.

Like conditioning, generalization can apply to positive stimuli. This is readily seen in human behavior (for example, if you fall in love with a person from a different country, it is easy to develop a liking for many things related to that country).

Generalization may be assisted by general enhanced excitability of amygdala neurons due to conditioning. Indeed, the tonic activity of both PKCDs and non-PKCDs increases after conditioning [Ciocchi et al., 2010]. The greater excitability of the PKCDs is due to a reduced inhibition in the extrasynaptic GABAA receptor alpha5 subunit, and PKCD stimulation promotes generalization [Botta et al., 2015].

Extinction. The conditioning-induced association between a CS and an innate can be subsequently 'weakened' by having the CS participate in quacia that do not involve the innate. The result of this extinction process is that the CS no longer activates the innate [Maren and Holmes, 2016, Pape and Pare, 2010]. Extinction does not necessarily reduce the strength of the original association, but it enables effective competition with it.

Most extinction protocols involve inputs having neutral valence, as opposed to other, competing innates (e.g., being in a certain spatial context without receiving footshock). If

\footnotetext{
${ }^{74}$ These are rodent terms. Approximate human homologues of IL and PL are the subgenual cingulate (BA25) and the dorsal anterior cingulate (BA32), respectively.
} 
the animal is in a situation that strengthens the competitive force of the original innate (e.g., anxiety), it may win the competition and overcome extinction. This is why stress can cause renewal of the original response, a phenomenon known as relapse or reinstatement.

\subsection{EEG oscillations}

The EEG shows oscillations in various frequency bands, especially during external execution. Oscillations reflect the activity of many neurons and are a natural manifestation of executing quacia. They are generally interrupted by alerts, a phenomenon known as eventrelated desynchronization (ERD). There have been attempts to associate oscillations in a given frequency band with specific cognitive operations. The R17 view is that frequency is affected by several general factors, including $\mathrm{R}$ mode, sensory input frequency, and area.

Oscillations are stronger with stronger neural firing. Thus, higher frequency oscillations are associated with more effortful R modes, especially acute focused execution. Sensory input can possess inherent spatial and/or temporal frequency shown by the flow it generates. Sensory (BU) input generates stronger flow than TD flow, so we can expect internal cognition to involve slower frequencies. Area affects frequency also because nodes in higher level areas are less susceptible to small surprise during execution. Hence, frontal regions usually exhibit slower rhythms (theta), while posterior regions exhibit faster ones (gamma).

Since oscillation frequency is area-dependent, and since the quax combines nodes from different areas, quacia normally exhibit various correlated frequencies (cross-frequency coupling (CFC)). Indeed, quacia that are more functionally effective (e.g., encode memory better) are associated with increased CFC between frontal and posterior regions [Friese et al., 2013], and fast posterior BU gamma from V1 to V4 is enhanced by slower TD beta from parietal area 7a to V1 [Richter et al., 2017].

Frequencies cannot be associated with cortical layers reliably in a task-independent manner. The superficial layers participate in acute responses, so can exhibit high frequencies. However, the superficial-deep layer balance is higher during internal cognition than during external cognition (because L2/3 is an internal network), so the superficial layers can also be associated with slower oscillations. For example, SOM and PV IINs, which generally coordinate superficial and deep pyramidal neurons respectively, support beta and gamma band oscillations in V1 (respectively) [Chen et al., 2017].

The gamma $(30-100 \mathrm{~Hz})$ band is associated with effortful, attended responses, and can also be promoted by high frequency inputs. For example, visual attention to an object exhibits gamma synchrony between superficial layers of the frontal eye fields (PFC) and visual area V4 [Gregoriou et al., 2012]. Gamma is naturally promoted by the PV basket IINs of the execution coordination network, as noted above. The beta $(15-30 \mathrm{~Hz})$ band is associated with motor pre-movement (e.g., posture, wait) motor actions [Aumann and Prut, 2015] and with TD flow [Kopell et al., 2010]. The theta (4-8Hz) band has two main instances. First, cortical (midline) theta is the rhythm of higher frontal nodes. Using human intracranial recordings, it was shown to be generated by alternating inputs into middle layers and feedback into superficial layers [Halgren et al., 2015]. Second, the rodent hippocampal theta rhythm is associated with locomotion, as discussed above. Oscillations in the delta $(1-4 \mathrm{~Hz})$ band are also encountered during wakefulness, but are less common.

The alpha $(8-15 \mathrm{~Hz})$ band is associated with three prominent phenomena. First, there is occipital alpha, which is associated with relaxed wakefulness, is strongest when the eyes are closed, and is replaced by slower $(3-8 \mathrm{~Hz})$ oscillations during sleep. Second, there is posterior alpha, which is stronger in areas that are not attended. It disintegrates during attention, showing ERD. It is very similar to the so-called Rolandic mu rhythm in sen- 
sorimotor cortex, which is shown during relaxed wakefulness but is diminished by tactile stimuli and motor activity. Third, there are alpha oscillations associated with increased attention, e.g., in the form of working memory [Foster et al., 2016]. All cases are explained by alpha being an internal rhythm diminished when attending external stimuli.

Occipital alpha may reflect the brain's processing of the visual background. We are all aware of the existence of a visual background during all of our activities (attended or automatic). This visual background is highly internally synchronized, and objects in it that move or that violate normal predictions can trigger the orienting of attention. The specific thalamic nucleus projecting to V1, the LGN, contains a population of cells inherently pulsing at alpha and connected by gap junctions [Hughes and Crunelli, 2007]. Recall that cortical L6 projects to the thalamic core network, completing a cycle that can drive precise synchrony. Cortical projections can yield attentional effects in the LGN [Saalmann and Kastner, 2014], showing that precise predictions affect all visual stations such that any unexpected motion would very quickly generate alerts.

\subsection{Attentional paradigms}

The attentional blink (AB). When a sequence of sensory targets is presented in rapid succession (3-10 per second), subjects usually do not report seeing the second of a successive pair of pre-specified targets, a phenomenon called the attentional blink (AB) [Kawahara et al., 2006]. The AB supports the $\mathrm{R} 17$ account of the $\mathrm{Q}$ process as the basic processing unit, and reporting as the basis for awareness. The task is a conditional wait, which involves the activation of the given targets in internal mode (probably prediction mode, because presentation is rapid). When the first target is identified, there is a transition from wait to execution of the conditional's reporting action (e.g., a button press). As in all transitions, some of the nodes implementing the previous state (the condition) are suppressed. If the second target arrives before higher level task nodes manage to reactivate these nodes, it is not reported. If the second target arrives while the brain executes the reporting task, the flow generated by it may be suppressed by competition, again with the result that it is not reported. In both cases, reporting is equivalent to awareness. The issue lies with the execution of the reporting action (following goal attainment) and not just with the speed of presentation, since spoken and written language can be understood at these paces.

A related phenomenon is repetition blindness, where people cannot keep track of how many times a target appears in a rapid sequence.

The brain can execute several tasks at the same time, but only one of them can be attended. This bottleneck may be due to the BG architecture, since cortico-STR-GPi/SNr projections exhibit large convergence, and competition in the vSTR may allow only a single motivated task. Several experimental paradigms exploit this property of the brain.

Inattentional blindness (IB). When subjects effortfully attend a task, they can fail to report salient simultaneous sensory events that would normally be noted (e.g., the famous gorilla appearing in a basketball scene [Chabris and Simons, 2010]). The flow generated by the sensory event does not make contact with non-automated reporting actions because it does not succeed in penetrating the running quax, which is busy with the attended task.

The Stroop effect. In target reporting experiments, reaction time is larger and there are more errors when the target has features that are normally associated with competing targets (for example, when the word 'blue' is printed in red color). This so-called Stroop effect is easily explained by $\mathrm{Q}$ process competition between the flow streams generated by the 
different features, which slows down automated processing.

Attentional cueing. In the well-known Posner paradigm, performance is better when a target appears at a pre-cued location. Cueing excites the DM network in a limited area, and this makes the response to objects appearing in this area faster.

Other phenomena having a similar nature as the ones above are the psychological refractory period and the inhibition of return.

\section{Discussion}

The brain is widely viewed as an enigma. Many partial accounts for specific phenomena have been proposed, but no comprehensive theory for brain function has yet emerged. Here I presented the first such theory. In this final section I discuss the nature of the theory, the weight of the evidence supporting it, its relationship with existing well-known notions, theory predictions and future work.

Nature of theory. As a general scientific theory, R17 has several positive properties. First, it is comprehensive, accounting for virtually all of the relevant important behavioral facts (due to length, learning and disorders are presented elsewhere). Second, it is supported by converging evidence from multiple modalities. Third, it is not contradicted by any existing evidence. Fourth, it is conceptually simple, phrased in terms of a single basic process. Finally, it expands its central high level processual description into a detailed concrete mechanistic account, thereby allowing the integration of experimental measurements and the formulation of theory predictions.

As a brain theory, R17 has additional positive aspects. First, it explains how brain function can arise from neurons and connections, without relying on any higher level management capacity. It does not use phrasings such as 'area $\mathrm{X}$ mediates function $\mathrm{Y}$ ' without first giving a precise definition of what it means for an area to mediate a function and how this arises from neuron-induced energy flow. Second, it is a biological theory, tightly connected to the needs of the organism and to its general state. Accounts phrased in other terms (e.g., cognitive, computational) can be easily misled by false metaphors and analogies and are not naturally suitable for bridging the gap with the biological instantiation. Brain theories should be especially wary of using words that have common everyday meanings (e.g., 'predictions'), because such words tend to divert the discussion from the concrete level to an excessively abstract ontological one. Third, the central notions in R17 are processes, not equations, which fits its goal of explaining a complex biological system.

Any theory claiming to explain brain function must show that it can plausibly explain all of the important aspects of brain function. The brain is a single organ, and omitting to explain a major capacity casts doubt on the explanations that are given. For example, a theory that addresses external movement but says nothing about imagery is akin to a theory of heart function that explains how the heart pumps blood out but not how blood gets in. This is why this paper includes aspects of the brain for which there is a smaller amount of evidence. Some readers may feel that it addresses too much material, but taking topics out would weaken the overall claim.

That this paper is comprehensive does not mean that all of the explanations it presents are correct. It is certainly possible that the overall framework and many specific accounts are correct, while some specific accounts are partially or fully incorrect. For example, consider the novel idea that SMA/preSMA use gMTNs to prime movement during imagery. This idea is consistent with the R17 imagery account, it provides a plausible reason for the existence of gMTNs, and it is supported by some converging evidence. However, it could 
also be wrong, and I am not aware of direct evidence supporting it. Nonetheless, even if it is wrong, this would not impair R17 as a brain theory in any serious way, because it is not essential for almost all parts of the theory.

Evidence. At present, many types of modalities provide large amounts of information about the brain, including methods from anatomy, physiology, pharmacology, imaging, EEG, lesion studies, genetics, and behavior. However, none of these methods provides information that is both spatially and temporally precise and covers a large number of neurons at large distances. As a result, current evidence provides direct support for some aspects of R17, but only indirect support for others. Specifically, there is good support for the general $\mathrm{N}$ mode sequence, the pairing of Rgens with $\mathrm{N}$ modes and cognitive functions, the existence and roles of the cortical flow networks, and center-surround competition, but only indirect support for the existence of the quax and our account of quax transitions and hierarchical action sequences. Nonetheless, there is a large amount of converging evidence to support these notions. Moreover, the fact that the better-understood immune system operates along lines generally similar to the $R$ process $[\mathrm{Paul}, 2013$ ] provides a strong argument in favor of R17. Comparing the situation to another major biological theory, evolution, the situation of brain theories is incomparably better. Here, we can perform a wider range of experiments, and it is reasonable to expect that measurement techniques would improve to provide increasingly more detailed information.

In most aspects of brain function, the weight of converging evidence is large enough to have allowed us to present a single dominant account. In some areas the situation is not that fortunate. For example, we could provide two alternatives for the details of how imagery (thinking) is implemented, but we could not definitely argue for any one of them.

Relationship with existing views. Virtually all of the central notions in R17 are novel, not having been recognized or discussed at all by previous work. These include the $\mathrm{R}$ process as a general process governing brain function, the $\mathrm{N}$ process, the management of $\mathrm{N}$ process modes by Rgens, the existence and functional roles of the flow and coordination networks, and the mechanistic account of $\mathrm{Q}$ process transitions and how it explains action sequences, language, and thought. Obviously, the existence of different types of excitatory neurons, inhibitory neurons and neuromodulators has been noted. However, as far as I am aware, these have not been assembled into higher level explanatory models. Also completely novel are the R17 accounts of the striosomes, $\mathrm{MCH}$, non-specific thalamic nuclei core, and theta phase precession, and the view of opioids as promoting the fundamental innate responses.

Strikingly, R17 dramatically differs from the accepted views in several crucial aspects of brain function, most notably with respect to the BG, GABA, mirror neurons, language, and motor control. In many other cases, R17 views some accepted accounts as highly misleading, e.g., in the case of DA and SER. There are many notions where R17 does not contradict accepted views, but improves upon them by presenting sharper definitions, detailed mechanisms, and integration with more general processes. This is the case with the amygdala (including fear conditioning, generalization and extinction), thalamus, habenula, hippocampus, minicolumns, NE, AVP, CRH, histamine, ACh, BEND, OT, DYN, ENK, GCs, ECBs, predictions, awareness via reporting, emotions, imagery, and place cells.

Let us discuss a few examples, starting with the BG. Virtually all existing accounts view the indirect path as stopping movement, while the R17 view is exactly the opposite, that it is what supports effortful and automated execution. In addition, the BG have been described as supporting reward, learning, motor actions, habits, chunking, focused selection and inhibition of competing motor programs, action selection, action reinforcement, switching from automatic to controlled behavior, and more. R17 agrees with these descrip- 
tions, but maintains that each of them presents just a partial facet of BG function.

A similar situation holds with respect to GABA. Inhibition is known to sculpt neural activity, which partially overlaps with R17's notion of coordination networks. However, GABA is most commonly described as providing 'inhibition'. The R17 view is that while technically correct (most of the time), this description is highly misleading, since the functional role of GABA is to support acute focused execution. When provided exogenously beyond the normal physiological doses, GABA can certainly suppress brain activity, but this does not say much about its normal functional role in brain processing ${ }^{75}$.

Regarding general brain anatomy, there is a general view that cortex represents higher cognition while subcortex represents more primitive or basic functions. The R17 account of two major subcortical structures, the thalamus and BG, is completely opposite to this common view, because they cooperate with cortex to jointly support adaptive responses but do not support innate ones.

With respect to mirror neurons, these have been given many exciting interpretations [Rizzolatti and Sinigaglia, 2008]. In R17, they are explained by a simple account that naturally arises from the nature of the $\mathrm{Q}$ process and content representations.

With respect to language, the academic world is polarized. There is a linguistics movement known as 'generative grammar' that has completely dominated linguistics research until very recently, and an opposing camp that generally takes a cognitive perspective. R17 views basically all of the tenets of generative linguistics as being totally wrong (see the supplementary material). At a very high level, R17 agrees with the cognitive linguistics camp about the nature of linguistic representations. However, their ideas have not been translated into a convincing brain theory. There are ideas from neuroscience that are compatible with some aspects of R17 (e.g., [Fiebach and Schubotz, 2006] view Broca's area as a premotor area), but all attempts that I am aware of for a more general theory (e.g., see [Hickok and Small, 2015]) are too vague.

Regarding motor control, the dominant accounts are inspired by control theory and view the brain as implementing forward or inverse models for optimal control. The Q process shows how these notions are integrated into a coherent whole, since its TD goaling can be viewed as the specification part of an inverse model, while its BU action triggering can be viewed as a forward model. In addition, optimality is not a central notion and is not guaranteed, as discussed at the end of the Q process section.

Dopamine has been described as indicating reward prediction error, general prediction error, motivation, effort, and hedonic pleasure. R17 agrees that DA is indeed involved with these. However, each of these descriptions is misleading, because they all stem from DA's general role in the $\mathrm{N}$ process, and contradict or omit other aspects of DA function. Especially misleading is the widely popular notion of DA as the brain's pleasure and reward chemical, since although DA is amplified during pleasure, it can be involved in highly nonpleasurable events (e.g., charging actions when fighting predators). Hence, we argue that the widely used term 'the reward system' is incorrect. In addition to DA's N process role, R17 also details the mechanistic aspects of DA's function, most of which not addressed by previous work (for example, I am not aware of any previous work that examined what exactly induces DA release during surprise).

Serotonin is widely believed to induce happiness. However, the fact that drugs that enhance SER presence in the brain (SSRIs) are used as antidepressants does not mean that SER yields happiness. In fact, the situation can be argued to be the opposite, since SER quenches emotions and promotes ruminations (thinking). SER may be useful in giving

\footnotetext{
${ }^{75} \mathrm{~A}$ similar argument can be made to critically criticize many conclusions drawn from experiments using optogenetic stimulation.
} 
people the opportunity of moving from a depressed to a more positive state, but it does not directly induce positive feelings. The best way to describe the role of SER (and other Rgens) is as promoting an N process mode (DM). Both its normal effects and its effects when given exogenously (e.g., enhancing creativity in small doses) stem from this role.

Finally, there are many notions that overlap with some aspects of R17 but address specific areas without sufficient detail or generalization. These include Hebb's cell assemblies, biased competition [Desimone and Duncan, 1995], the action perception cycle [Fuster, 2015], predictive coding [Adams et al., 2013], reverse hierarchy theory [Ahissar and Hochstein, 2004], dual-process theories [Kahneman, 2011], awareness and emotions. Some theories view predictions as the major principle of brain function [Clark, 2015], while in R17 they are just one aspect of R process execution. Similarly, our view of the hippocampus as representing arbitrary new acute events is a natural generalization of its role in spatial navigation and memory [Buzsáki and Moser, 2013, Moscovitch et al., 2016], but its mechanistic function is much easier to understand with the R17 observation that its subfields belong to the $\mathrm{R}$ process flow networks. In the well-known stress response, stressors excite GCs via CRH and pituitary ACTH, and GCs exert negative feedback to suppress the HPA axis. R17 agrees that this process occurs, but views it as a relatively small part of the acute response, constituting a single $\mathrm{N}$ process mode, one type of termination.

Falsification and theory predictions. R17 gives a precise description of the role of Rgens and of the sequence of neuronal activation in various execution modes. It is thus inherently falsifiable and can be used to derive a large number of theory predictions. Any technique that measures neurons of a specific type and discovers a consistently different sequence would refute the relevant parts of R17. For example, if a node's response network neurons would turn out to be activated before prediction network neurons during attended surpriseless execution, this would falsify Q process goaling. If controlled and automated execution would turn out to use identical patterns of cortical activation and differ only in the BG or thalamus, this would falsify the R17 account of automaticity and/or competition. If BEND, ACh or any other Rgen would turn out to be substantially released in situations that do not conform with the $\mathrm{N}$ modes assigned to it by $\mathrm{R} 17$, some of the $\mathrm{N}$ process would be falsified. Naturally, such measurements may not be easy to do; nonetheless, modern measurement techniques such as two-photon imaging, which can capture single dendritic spines, can already provide data at a sufficient resolution.

It is informative to highlight a theory prediction in the area at which R17 is least detailed, dynamic imagery. Even though we could not present a definitive account of the activation mode of the imagined object nodes, we still presented a detailed prediction of many aspects of how imagery is implemented, including inter-node flow, transitions, sequencing, hierarchy, $\mathrm{N}$ mode, supporting Rgen, and major areas. Any of these can be refuted. For example, if brains are measured and it is discovered that imagery is not managed by a $\mathrm{Q}$ process, this part of R17 would be falsified.

Future work. R17 is a detailed theory of brain function, but it is still a high level theory. In my opinion, this is the level at which a theory of brain function should be phrased. Nonetheless, there is an endless depth of details that need to be further elaborated, as in all scientific theories. An important high level direction for future work is to implement brain simulations based on R17. Simulations with a non-trivial number of neurons and nodes are certainly feasible with today's technology.

Several important issues were not fully settled in this paper, including whether nodes are unique representing sets and the activation mode of imagined object nodes. Many topics merit a longer discussion, including the exact representation of time, the areal location of 
specific content types, the mechanistic links between the different types of space-related cells, and volition. There are some aspects of brain anatomy and function that were not addressed at all, for example von Economo neurons, the zona incerta, and Rgens such as galanin and neuropeptide $\mathrm{S}$.

Several important topics have already been elaborated in R17, but are described in different publications due to length constraints. These include brain learning (including development and sleep), the food system, the sex system (including gender differences), the brain's relationship with the metabolism and immune systems, genetics (including epigenetics), and brain disorders

\section{Final Words}

This paper is admittedly ambitious, presenting a single theory that claims to answer most of the fundamental questions about cognition and the brain that have fascinated humanity for thousands of years. I think that the theory is strongly supported by the available factual evidence and by its simplicity and self consistency. Errors and omissions are inevitable in a work of this scope, but hopefully the remaining ones are relatively minor and easy to fix. In any event, I hope that R17 inspires other people to engage in similar efforts, to the betterment of humankind.

\section{References}

[Adams et al., 2011] Adams, A. C., Domouzoglou, E. M., Chee, M. J., Segal-Lieberman, G., Pissios, P., and Maratos-Flier, E. (2011). Ablation of the hypothalamic neuropeptide melanin concentrating hormone is associated with behavioral abnormalities that reflect impaired olfactory integration. Behavioural brain research, 224(1):195-200.

[Adams et al., 2013] Adams, R. A., Shipp, S., and Friston, K. J. (2013). Predictions not commands: active inference in the motor system. Brain Structure and Function, 218(3):611-643.

[Adolphs, 2015] Adolphs, R. (2015). The unsolved problems of neuroscience. Trends in cognitive sciences, 19(4):173-175.

[Aggleton, 2012] Aggleton, J. P. (2012). Multiple anatomical systems embedded within the primate medial temporal lobe: implications for hippocampal function. Neuroscience \& Biobehavioral Reviews, 36(7):1579-1596.

[Agnoli et al., 2013] Agnoli, L., Mainolfi, P., Invernizzi, R. W., and Carli, M. (2013). Dopamine D1-like and D2-like receptors in the dorsal striatum control different aspects of attentional performance in the five-choice serial reaction time task under a condition of increased activity of corticostriatal inputs. Neuropsychopharmacology, 38(5):701714.

[Ahissar and Hochstein, 2004] Ahissar, M. and Hochstein, S. (2004). The reverse hierarchy theory of visual perceptual learning. Trends in cognitive sciences, 8(10):457-464.

[Alberini and LeDoux, 2013] Alberini, C. M. and LeDoux, J. E. (2013). Memory reconsolidation. Current Biology, 23(17):R746-R750. 
[Albuquerque et al., 2009] Albuquerque, E. X., Pereira, E. F., Alkondon, M., and Rogers, S. W. (2009). Mammalian nicotinic acetylcholine receptors: from structure to function. Physiological reviews, 89(1):73-120.

[Alegre et al., 2010] Alegre, M., Rodríguez-Oroz, M., Valencia, M., Pérez-Alcázar, M., Guridi, J., Iriarte, J., Obeso, J., and Artieda, J. (2010). Changes in subthalamic activity during movement observation in Parkinson's disease: is the mirror system mirrored in the basal ganglia? Clinical Neurophysiology, 121(3):414-425.

[Allen et al., 2012] Allen, K., Pereira, F., Botvinick, M., and Goldberg, A. E. (2012). Distinguishing grammatical constructions with fMRI pattern analysis. Brain and Language, 123(3):174-182.

[Alunni-Menichini et al., 2014] Alunni-Menichini, K., Guimond, S., Bermudez, P., Nolden, S., Lefebvre, C., and Jolicoeur, P. (2014). Saturation of auditory short-term memory causes a plateau in the sustained anterior negativity event-related potential. Brain research, 1592:55-64.

[Anstrom et al., 2009] Anstrom, K. K., Miczek, K. A., and Budygin, E. A. (2009). Increased phasic dopamine signaling in the mesolimbic pathway during social defeat in rats. Neuroscience, 161(1):3-12.

[Antoniadis and McDonald, 2001] Antoniadis, E. A. and McDonald, R. J. (2001). Amygdala, hippocampus, and unconditioned fear. Experimental Brain Research, 138(2):200209.

[Aoyama and Kaneko, 2011] Aoyama, T. and Kaneko, F. (2011). The effect of motor imagery on gain modulation of the spinal reflex. Brain research, 1372:41-48.

[Arnsten, 2013] Arnsten, A. (2013). The neurobiology of thought: the groundbreaking discoveries of Patricia Goldman-Rakic 1937-2003. Cerebral Cortex, 23(10):2269-2281.

[Arnsten and Pliszka, 2011] Arnsten, A. F. and Pliszka, S. R. (2011). Catecholamine influences on prefrontal cortical function: relevance to treatment of attention deficit/hyperactivity disorder and related disorders. Pharmacology Biochemistry and Behavior, 99(2):211-216.

[Asahina et al., 2014] Asahina, K., Watanabe, K., Duistermars, B. J., Hoopfer, E., González, C. R., Eyjólfsdóttir, E. A., Perona, P., and Anderson, D. J. (2014). Tachykininexpressing neurons control male-specific aggressive arousal in drosophila. Cell, 156(1):221-235.

[Atwood et al., 2014] Atwood, B. K., Kupferschmidt, D. A., and Lovinger, D. M. (2014). Opioids induce dissociable forms of long-term depression of excitatory inputs to the dorsal striatum. Nature neuroscience, 17(4):540-548.

[Aumann and Prut, 2015] Aumann, T. D. and Prut, Y. (2015). Do sensorimotor $\beta$ oscillations maintain muscle synergy representations in primary motor cortex? Trends in neurosciences, 38(2):77-85.

[Avesar and Gulledge, 2012] Avesar, D. and Gulledge, A. T. (2012). Selective serotonergic excitation of callosal projection neurons. Frontiers in neural circuits, 6:12. 
[Azmitia, 2010] Azmitia, E. C. (2010). .1-evolution of serotonin: Sunlight to suicide. Handbook of Behavioral Neuroscience, 21:3-22.

[Backholer et al., 2010] Backholer, K., Smith, J. T., Rao, A., Pereira, A., Iqbal, J., Ogawa, S., Li, Q., and Clarke, I. J. (2010). Kisspeptin cells in the ewe brain respond to leptin and communicate with neuropeptide $\mathrm{Y}$ and proopiomelanocortin cells. Endocrinology, 151(5):2233-2243.

[Bailey et al., 2018] Bailey, M. R., Goldman, O., Bello, E. P., Chohan, M. O., Jeong, N., Winiger, V., Chun, E., Schipani, E., Kalmbach, A., Cheer, J. F., et al. (2018). An interaction between serotonin receptor signaling and dopamine enhances goal-directed vigor and persistence in mice. Journal of Neuroscience, 38(9):2149-2162.

[Baimel and Borgland, 2015] Baimel, C. and Borgland, S. L. (2015). Orexin signaling in the VTA gates morphine-induced synaptic plasticity. Journal of Neuroscience, 35(18):7295-7303.

[Bains, 2014] Bains, J. S. (2014). Stress-induced metaplasticity at GABA synapses. In Synaptic Stress and Pathogenesis of Neuropsychiatric Disorders, pages 125-136. Springer.

[Baker et al., 2018] Baker, A. L., O’Toole, R. J., and Gulledge, A. T. (2018). Preferential cholinergic excitation of corticopontine neurons. The Journal of physiology.

[Balsevich et al., 2017] Balsevich, G., Petrie, G. N., and Hill, M. N. (2017). Endocannabinoids: Effectors of glucocorticoid signaling. Frontiers in Neuroendocrinology, 47:86108.

[Bar-Gad et al., 2003] Bar-Gad, I., Morris, G., and Bergman, H. (2003). Information processing, dimensionality reduction and reinforcement learning in the basal ganglia. Progress in neurobiology, 71(6):439-473.

[Basbaum et al., 2009] Basbaum, A. I., Bautista, D. M., Scherrer, G., and Julius, D. (2009). Cellular and molecular mechanisms of pain. Cell, 139(2):267-284.

[Ben-Ari, 2014] Ben-Ari, Y. (2014). The GABA excitatory/inhibitory developmental sequence: a personal journey. Neuroscience, 279:187-219.

[Benarroch, 2012] Benarroch, E. E. (2012). GABAB receptors structure, functions, and clinical implications. Neurology, 78(8):578-584.

[Benarroch, 2015] Benarroch, E. E. (2015). Habenula recently recognized functions and potential clinical relevance. Neurology, 85(11):992-1000.

[Blot and Barbour, 2014] Blot, A. and Barbour, B. (2014). Ultra-rapid axon-axon ephaptic inhibition of cerebellar Purkinje cells by the pinceau. Nature neuroscience, 17(2):289295.

[Blouin et al., 2013] Blouin, A. M., Fried, I., Wilson, C. L., Staba, R. J., Behnke, E. J., Lam, H. A., Maidment, N. T., Lapierre, J. L., Siegel, J. M., et al. (2013). Human hypocretin and melanin-concentrating hormone levels are linked to emotion and social interaction. Nature communications, 4:1547. 
[Bonifacci et al., 2015] Bonifacci, P., Desideri, L., and Ottaviani, C. (2015). Familiarity of faces: Sense or feeling? an exploratory investigation with eye movements and skin conductance. Journal of Psychophysiology.

[Borsook et al., 2014] Borsook, D., Hargreaves, R., Bountra, C., and Porreca, F. (2014). Lost but making progress - where will new analgesic drugs come from? Science translational medicine, 6(249):249sr3-249sr3.

[Bostan et al., 2013] Bostan, A. C., Dum, R. P., and Strick, P. L. (2013). Cerebellar networks with the cerebral cortex and basal ganglia. Trends in cognitive sciences, 17(5):241-254.

[Botta et al., 2015] Botta, P., Demmou, L., Kasugai, Y., Markovic, M., Xu, C., Fadok, J. P., Lu, T., Poe, M. M., Xu, L., Cook, J. M., et al. (2015). Regulating anxiety with extrasynaptic inhibition. Nature neuroscience, 18(10):1493-1500.

[Brandt et al., 2016] Brandt, K. R., Eysenck, M. W., Nielsen, M. K., and von Oertzen, T. J. (2016). Selective lesion to the entorhinal cortex leads to an impairment in familiarity but not recollection. Brain and cognition, 104:82-92.

[Brighetti et al., 2007] Brighetti, G., Bonifacci, P., Borlimi, R., and Ottaviani, C. (2007). "far from the heart far from the eye": evidence from the capgras delusion. Cognitive Neuropsychiatry, 12(3):189-197.

[Brinton, 2008] Brinton, R. D. (2008). The healthy cell bias of estrogen action: mitochondrial bioenergetics and neurological implications. Trends in neurosciences, 31(10):529_ 537.

[Brombas et al., 2014] Brombas, A., Fletcher, L. N., and Williams, S. R. (2014). Activitydependent modulation of layer 1 inhibitory neocortical circuits by acetylcholine. Journal of Neuroscience, 34(5):1932-1941.

[Bromberg-Martin et al., 2010] Bromberg-Martin, E. S., Matsumoto, M., and Hikosaka, O. (2010). Dopamine in motivational control: rewarding, aversive, and alerting. Neuron, 68(5):815-834.

[Brown et al., 2015] Brown, J. A., Woodworth, H. L., and Leinninger, G. M. (2015). To ingest or rest? specialized roles of lateral hypothalamic area neurons in coordinating energy balance. Frontiers in systems neuroscience, 9:9.

[Brown, 2014] Brown, J. W. (2014). The tale of the neuroscientists and the computer: why mechanistic theory matters. Frontiers in neuroscience, 8.

[Brunia et al., 2012] Brunia, C., van Boxtel, G., Böcker, K., Kappenman, E., and Luck, S. (2012). Negative slow waves as indices of anticipation: The bereitschaftspotential, the contingent negative variation, and the stimulus-preceding negativity. The Oxford handbook of event-related potential components, pages 189-207.

[Buzsaki, 2006] Buzsaki, G. (2006). Rhythms of the Brain. Oxford University Press.

[Buzsáki, 2015] Buzsáki, G. (2015). Hippocampal sharp wave-ripple: A cognitive biomarker for episodic memory and planning. Hippocampus, 25(10):1073-1188. 
[Buzsáki and Moser, 2013] Buzsáki, G. and Moser, E. I. (2013). Memory, navigation and theta rhythm in the hippocampal-entorhinal system. Nature neuroscience, 16(2):130 138.

[Cachope et al., 2012] Cachope, R., Mateo, Y., Mathur, B. N., Irving, J., Wang, H.-L., Morales, M., Lovinger, D. M., and Cheer, J. F. (2012). Selective activation of cholinergic interneurons enhances accumbal phasic dopamine release: setting the tone for reward processing. Cell reports, 2(1):33-41.

[Cai et al., 2014] Cai, H., Haubensak, W., Anthony, T. E., and Anderson, D. J. (2014). Central amygdala PKC-[delta]+ neurons mediate the influence of multiple anorexigenic signals. Nature neuroscience, 17(9):1240-1248.

[Cain and Cidlowski, 2017] Cain, D. W. and Cidlowski, J. A. (2017). Immune regulation by glucocorticoids. Nature Reviews Immunology, 17(4):233-247.

[Caplan and Waters, 1999] Caplan, D. and Waters, G. S. (1999). Verbal working memory and sentence comprehension. Behavioral and brain Sciences, 22(1):77-94.

[Cardoso et al., 2012] Cardoso, M. M., Sirotin, Y. B., Lima, B., Glushenkova, E., and Das, A. (2012). The neuroimaging signal is a linear sum of neurally distinct stimulus-and task-related components. Nature neuroscience, 15(9):1298-1306.

[Carr, 2015] Carr, J. A. (2015). I'll take the low road: the evolutionary underpinnings of visually triggered fear. Frontiers in neuroscience, 9.

[Carter, 2014] Carter, C. S. (2014). Oxytocin pathways and the evolution of human behavior. Annual review of psychology, 65:17-39.

[Carter et al., 2015] Carter, M. E., Han, S., and Palmiter, R. D. (2015). Parabrachial calcitonin gene-related peptide neurons mediate conditioned taste aversion. The Journal of neuroscience, 35(11):4582-4586.

[Castro and Berridge, 2014] Castro, D. C. and Berridge, K. C. (2014). Opioid hedonic hotspot in nucleus accumbens shell: mu, delta, and kappa maps for enhancement of sweetness "liking" and "wanting". The Journal of Neuroscience, 34(12):4239-4250.

[Cei et al., 2014] Cei, A., Girardeau, G., Drieu, C., El Kanbi, K., and Zugaro, M. (2014). Reversed theta sequences of hippocampal cell assemblies during backward travel. $\mathrm{Na}$ ture neuroscience, 17(5):719-724.

[Chabris and Simons, 2010] Chabris, C. and Simons, D. (2010). The invisible gorilla: And other ways our intuitions deceive us. Harmony.

[Chen et al., 2017] Chen, G., Zhang, Y., Li, X., Zhao, X., Ye, Q., Lin, Y., Tao, H. W., Rasch, M. J., and Zhang, X. (2017). Distinct inhibitory circuits orchestrate cortical beta and gamma band oscillations. Neuron, 96(6):1403-1418.

[Chen et al., 2015] Chen, Q., Lecea, L., Hu, Z., and Gao, D. (2015). The hypocretin/orexin system: an increasingly important role in neuropsychiatry. Medicinal research reviews, 35(1):152-197. 
[Chen et al., 2011] Chen, X., Gabitto, M., Peng, Y., Ryba, N. J., and Zuker, C. S. (2011). A gustotopic map of taste qualities in the mammalian brain. Science, 333(6047):12621266.

[Cheng, 2013] Cheng, M.-F. (2013). Hypothalamic neurogenesis in the adult brain. Frontiers in neuroendocrinology, 34(3):167-178.

[Cheong and Shin, 2013] Cheong, E. and Shin, H.-S. (2013). T-type Ca2+ channels in normal and abnormal brain functions. Physiological reviews, 93(3):961-992.

[Christian et al., 2014] Christian, K. M., Song, H., and Ming, G.-1. (2014). Functions and dysfunctions of adult hippocampal neurogenesis. Annual review of neuroscience, 37:243-262.

[Ciocchi et al., 2010] Ciocchi, S., Herry, C., Grenier, F., Wolff, S. B., Letzkus, J. J., Vlachos, I., Ehrlich, I., Sprengel, R., Deisseroth, K., Stadler, M. B., et al. (2010). Encoding of conditioned fear in central amygdala inhibitory circuits. Nature, 468(7321):277-282.

[Cisek and Pastor-Bernier, 2014] Cisek, P. and Pastor-Bernier, A. (2014). On the challenges and mechanisms of embodied decisions. Phil. Trans. R. Soc. B, 369(1655):20130479.

[Clark, 2015] Clark, A. (2015). Surfing uncertainty: Prediction, action, and the embodied mind. Oxford University Press.

[Clarkson et al., 2017a] Clarkson, J., Han, S. Y., Piet, R., McLennan, T., Kane, G. M., Ng, J., Porteous, R. W., Kim, J. S., Colledge, W. H., Iremonger, K. J., et al. (2017a). Definition of the hypothalamic gnrh pulse generator in mice. Proceedings of the National Academy of Sciences, page 201713897.

[Clarkson et al., 2017b] Clarkson, R. L., Liptak, A. T., Gee, S. M., Sohal, V. S., and Bender, K. J. (2017b). D3 receptors regulate excitability in a unique class of prefrontal pyramidal cells. Journal of Neuroscience, 37(24):5846-5860.

[Clascá et al., 2012] Clascá, F., Rubio-Garrido, P., and Jabaudon, D. (2012). Unveiling the diversity of thalamocortical neuron subtypes. European Journal of Neuroscience, 35(10):1524-1532.

[Cole and Paillard, 1995] Cole, J. and Paillard, J. (1995). Living without touch and peripheral information about body position and movement: Studies with deafferented subjects. The body and the self, pages 245-266.

[Constantinople and Bruno, 2013] Constantinople, C. M. and Bruno, R. M. (2013). Deep cortical layers are activated directly by thalamus. Science, 340(6140):1591-1594.

[Coslovich et al., 2017] Coslovich, T., Brumley, M. R., D’Angelo, G., Della Mora, A., Swann, H. E., Ortolani, F., and Taccola, G. (2017). Histamine modulates spinal motoneurons and locomotor circuits. Journal of Neuroscience Research.

[Craig, 2014] Craig, A. (2014). How do you feel?: an interoceptive moment with your neurobiological self. Princeton University Press.

[Cristino et al., 2014] Cristino, L., Becker, T., and Marzo, V. (2014). Endocannabinoids and energy homeostasis: an update. Biofactors, 40(4):389-397. 
[Crittenden and Graybiel, 2011] Crittenden, J. R. and Graybiel, A. M. (2011). Basal ganglia disorders associated with imbalances in the striatal striosome and matrix compartments. Frontiers in Neuroanatomy, 5:59.

[Cui et al., 2013] Cui, G., Jun, S. B., Jin, X., Pham, M. D., Vogel, S. S., Lovinger, D. M., and Costa, R. M. (2013). Concurrent activation of striatal direct and indirect pathways during action initiation. Nature, 494(7436):238-242.

[Czyrak et al., 2003] Czyrak, A., Czepiel, K., Maćkowiak, M., Chocyk, A., and Wędzony, K. (2003). Serotonin 5-HT1A receptors might control the output of cortical glutamatergic neurons in rat cingulate cortex. Brain research, 989(1):42-51.

[da Cunha-Bang et al., 2016] da Cunha-Bang, S., Mc Mahon, B., MacDonald Fisher, P., Jensen, P. S., Svarer, C., and Moos Knudsen, G. (2016). High trait aggression in men is associated with low 5-HT levels, as indexed by 5-HT 4 receptor binding. Social cognitive and affective neuroscience, 11(4):548-555.

[Damasio and Carvalho, 2013] Damasio, A. and Carvalho, G. B. (2013). The nature of feelings: evolutionary and neurobiological origins. Nature Reviews Neuroscience, 14(2):143-152.

[Danielmeier et al., 2015] Danielmeier, C., Allen, E. A., Jocham, G., Onur, O. A., Eichele, T., and Ullsperger, M. (2015). Acetylcholine mediates behavioral and neural post-error control. Current Biology, 25(11):1461-1468.

[Davare et al., 2015] Davare, M., Zénon, A., Desmurget, M., and Olivier, E. (2015). Dissociable contribution of the parietal and frontal cortex to coding movement direction and amplitude. Frontiers in human neuroscience, 9.

[Day et al., 1999] Day, H., Curran, E., Watson Jr, S., and Akil, H. (1999). Distinct neurochemical populations in the rat central nucleus of the amygdala and bed nucleus of the stria terminalis: evidence for their selective activation by interleukin-1beta. The Journal of comparative neurology, 413(1):113.

[De Deurwaerdère and Di Giovanni, 2017] De Deurwaerdère, P. and Di Giovanni, G. (2017). Serotonergic modulation of the activity of mesencephalic dopaminergic systems: therapeutic implications. Progress in neurobiology, 151:175-236.

[De Dreu and Kret, 2016] De Dreu, C. K. and Kret, M. E. (2016). Oxytocin conditions intergroup relations through upregulated in-group empathy, cooperation, conformity, and defense. Biological Psychiatry, 79(3):165-173.

[De Goede et al., 2009] De Goede, D., Shapiro, L. P., Wester, F., Swinney, D. A., and Bastiaanse, R. (2009). The time course of verb processing in Dutch sentences. Journal of psycholinguistic research, 38(3):181-199.

[DeFelipe et al., 2013] DeFelipe, J., López-Cruz, P. L., Benavides-Piccione, R., Bielza, C., Larrañaga, P., Anderson, S., Burkhalter, A., Cauli, B., Fairén, A., Feldmeyer, D., et al. (2013). New insights into the classification and nomenclature of cortical GABAergic interneurons. Nature Reviews Neuroscience, 14(3):202-216.

[Dehaene, 2014] Dehaene, S. (2014). Consciousness and the brain: Deciphering how the brain codes our thoughts. Penguin. 
[DeLong et al., 2014] DeLong, K. A., Troyer, M., and Kutas, M. (2014). Pre-processing in sentence comprehension: Sensitivity to likely upcoming meaning and structure. Language and Linguistics Compass, 8(12):631-645.

[Der-Avakian et al., 2017] Der-Avakian, A., D’Souza, M. S., Potter, D. N., Chartoff, E. H., Carlezon, W. A., Pizzagalli, D. A., and Markou, A. (2017). Social defeat disrupts reward learning and potentiates striatal nociceptin/orphanin FQ mRNA in rats. Psychopharmacology, 234(9-10):1603-1614.

[Desimone and Duncan, 1995] Desimone, R. and Duncan, J. (1995). Neural mechanisms of selective visual attention. Annual review of neuroscience, 18(1):193-222.

[Desmurget et al., 2009] Desmurget, M., Reilly, K. T., Richard, N., Szathmari, A., Mottolese, C., and Sirigu, A. (2009). Movement intention after parietal cortex stimulation in humans. science, 324(5928):811-813.

[Devera et al., 2015] Devera, A., Pascovich, C., Lagos, P., Falconi, A., Sampogna, S., Chase, M. H., and Torterolo, P. (2015). Melanin-concentrating hormone (MCH) modulates the activity of dorsal raphe neurons. Brain research, 1598:114-128.

[Di Sebastiano and Coolen, 2012] Di Sebastiano, A. and Coolen, L. (2012). Orexin and natural reward: feeding, maternal, and male sexual behavior. In Shekhar, A., editor, The Orexin/hypocretin System. Elsevier.

[DiFeliceantonio et al., 2012] DiFeliceantonio, A. G., Mabrouk, O. S., Kennedy, R. T., and Berridge, K. C. (2012). Enkephalin surges in dorsal neostriatum as a signal to eat. Current Biology, 22(20):1918-1924.

[Ding et al., 2010] Ding, J. B., Guzman, J. N., Peterson, J. D., Goldberg, J. A., and Surmeier, D. J. (2010). Thalamic gating of corticostriatal signaling by cholinergic interneurons. Neuron, 67(2):294-307.

[Ding and Zhou, 2014] Ding, S. and Zhou, F.-M. (2014). Serotonin regulation of subthalamic neurons. Reviews in the Neurosciences, 25(4):605-619.

[Dixon, 2005] Dixon, R. M. W. (2005). A semantic approach to English grammar. Oxford: Oxford University Press,, 2nd edition.

[Dölen et al., 2013] Dölen, G., Darvishzadeh, A., Huang, K. W., and Malenka, R. C. (2013). Social reward requires coordinated activity of nucleus accumbens oxytocin and serotonin. Nature, 501(7466):179-184.

[Domínguez et al., 2014] Domínguez, S., de Sevilla, D. F., and Buño, W. (2014). Postsynaptic activity reverses the sign of the acetylcholine-induced long-term plasticity of GABAA inhibition. Proceedings of the National Academy of Sciences, 111(26):E2741E2750.

[Donahue et al., 2015] Donahue, R. J., Landino, S. M., Golden, S. A., Carroll, F. I., Russo, S. J., and Carlezon Jr, W. A. (2015). Effects of acute and chronic social defeat stress are differentially mediated by the dynorphin/kappa-opioid receptor system. Behavioural pharmacology, $26(70$ 0):654. 
[Dorocic et al., 2014] Dorocic, I. P., Fürth, D., Xuan, Y., Johansson, Y., Pozzi, L., Silberberg, G., Carlén, M., and Meletis, K. (2014). A whole-brain atlas of inputs to serotonergic neurons of the dorsal and median raphe nuclei. Neuron, 83(3):663-678.

[Drew et al., 2009] Drew, G. M., Lau, B. K., and Vaughan, C. W. (2009). Substance P drives endocannabinoid-mediated disinhibition in a midbrain descending analgesic pathway. Journal of Neuroscience, 29(22):7220-7229.

[Dunbar et al., 2012] Dunbar, R., Kaskatis, K., MacDonald, I., and Barra, V. (2012). Performance of music elevates pain threshold and positive affect: implications for the evolutionary function of music. Evolutionary psychology: an international journal of evolutionary approaches to psychology and behavior, 10(4):688-702.

[Duque et al., 2013] Duque, J., Olivier, E., and Rushworth, M. (2013). Top-down inhibitory control exerted by the medial frontal cortex during action selection under conflict. Journal of cognitive neuroscience, 25(10):1634-1648.

[Ebner and Singewald, 2006] Ebner, K. and Singewald, N. (2006). The role of substance $\mathrm{P}$ in stress and anxiety responses. Amino acids, 31(3):251-272.

[Elston et al., 2006] Elston, G. N., Benavides-Piccione, R., Elston, A., Zietsch, B., Defelipe, J., Manger, P., Casagrande, V., and Kaas, J. H. (2006). Specializations of the granular prefrontal cortex of primates: implications for cognitive processing. The Anatomical Record, 288(1):26-35.

[Fanselow et al., 2008] Fanselow, E. E., Richardson, K. A., and Connors, B. W. (2008). Selective, state-dependent activation of somatostatin-expressing inhibitory interneurons in mouse neocortex. Journal of Neurophysiology, 100(5):2640-2652.

[Feng et al., 2012] Feng, Y., He, X., Yang, Y., Chao, D., Lazarus, L. H., and Xia, Y. (2012). Current research on opioid receptor function. Current drug targets, 13(2):230.

[Fergani and Navarro, 2017] Fergani, C. and Navarro, V. M. (2017). Expanding the role of tachykinins in the neuroendocrine control of reproduction. Reproduction, 153(1):R1R14.

[Ferguson and Gao, 2017] Ferguson, B. R. and Gao, W.-J. (2017). Thalamic control of cognition and social behavior via regulation of GABAergic signaling and E/I balance in the medial prefrontal cortex. Biological Psychiatry.

[Fernandes et al., 2014] Fernandes, C. C., Lozada, A. F., and Berg, D. K. (2014). Nicotinic signaling in development. In Nicotinic Receptors, pages 115-135. Springer.

[Ferrari et al., 2016] Ferrari, L., Agostinelli, L., Krashes, M., Lowell, B., Scammell, T., and Arrigoni, E. (2016). Dynorphin inhibits basal forebrain cholinergic neurons by preand postsynaptic mechanisms. The Journal of physiology, 594(4):1069-1085.

[Fiacconi et al., 2014] Fiacconi, C. M., Barkley, V., Finger, E. C., Carson, N., Duke, D., Rosenbaum, R. S., Gilboa, A., and Köhler, S. (2014). Nature and extent of person recognition impairments associated with capgras syndrome in lewy body dementia. Frontiers in human neuroscience, 8:726. 
[Fiebach and Schubotz, 2006] Fiebach, C. J. and Schubotz, R. I. (2006). Dynamic anticipatory processing of hierarchical sequential events: a common role for Broca's area and ventral premotor cortex across domains? Cortex, 42(4):499-502.

[Fields, 2007] Fields, H. L. (2007). Understanding how opioids contribute to reward and analgesia. Regional anesthesia and pain medicine, 32(3):242-246.

[Fields and Margolis, 2015] Fields, H. L. and Margolis, E. B. (2015). Understanding opioid reward. Trends in neurosciences, 38(4):217-225.

[Fink and Göthert, 2007] Fink, K. B. and Göthert, M. (2007). 5-HT receptor regulation of neurotransmitter release. Pharmacological reviews, 59(4):360-417.

[Fischbach-Weiss et al., 2017] Fischbach-Weiss, S., Reese, R. M., and Janak, P. H. (2017). Inhibiting mesolimbic dopamine neurons reduces the initiation and maintenance of instrumental responding. Neuroscience.

[Fish et al., 2013] Fish, K. N., Hoftman, G. D., Sheikh, W., Kitchens, M., and Lewis, D. A. (2013). Parvalbumin-containing chandelier and basket cell boutons have distinctive modes of maturation in monkey prefrontal cortex. Journal of Neuroscience, 33(19):8352-8358.

[Fonseca et al., 2015] Fonseca, M. S., Murakami, M., and Mainen, Z. F. (2015). Activation of dorsal raphe serotonergic neurons promotes waiting but is not reinforcing. Current Biology, 25(3):306-315.

[Forster et al., 2008] Forster, G. L., Pringle, R. B., Mouw, N. J., Vuong, S. M., Watt, M. J., Burke, A. R., Lowry, C. A., Summers, C. H., and Renner, K. J. (2008). Corticotropinreleasing factor in the dorsal raphe nucleus increases medial prefrontal cortical serotonin via type 2 receptors and median raphe nucleus activity. European Journal of Neuroscience, 28(2):299-310.

[Foster et al., 2016] Foster, J. J., Sutterer, D. W., Serences, J. T., Vogel, E. K., and Awh, E. (2016). The topography of alpha-band activity tracks the content of spatial working memory. Journal of neurophysiology, 115(1):168-177.

[Frank et al., 2015] Frank, S. L., Trompenaars, T., and Vasishth, S. (2015). Crosslinguistic differences in processing double-embedded relative clauses: Workingmemory constraints or language statistics? Cognitive science.

[Friederici, 2011] Friederici, A. D. (2011). The brain basis of language processing: from structure to function. Physiological reviews, 91(4):1357-1392.

[Friese et al., 2013] Friese, U., Köster, M., Hassler, U., Martens, U., Trujillo-Barreto, N., and Gruber, T. (2013). Successful memory encoding is associated with increased crossfrequency coupling between frontal theta and posterior gamma oscillations in human scalp-recorded EEG. Neuroimage, 66:642-647.

[Frith and Frith, 2005] Frith, C. and Frith, U. (2005). Theory of mind. Current Biology, 15(17):R644-R645.

[Fu et al., 2014] Fu, Y., Tucciarone, J. M., Espinosa, J. S., Sheng, N., Darcy, D. P., Nicoll, R. A., Huang, Z. J., and Stryker, M. P. (2014). A cortical circuit for gain control by behavioral state. Cell, 156(6):1139-1152. 
[Fujiyama et al., 2011] Fujiyama, F., Sohn, J., Nakano, T., Furuta, T., Nakamura, K. C., Matsuda, W., and Kaneko, T. (2011). Exclusive and common targets of neostriatofugal projections of rat striosome neurons: a single neuron-tracing study using a viral vector. European Journal of Neuroscience, 33(4):668-677.

[Fuss et al., 2015] Fuss, J., Steinle, J., Bindila, L., Auer, M. K., Kirchherr, H., Lutz, B., and Gass, P. (2015). A runner's high depends on cannabinoid receptors in mice. Proceedings of the National Academy of Sciences, 112(42):13105-13108.

[Fuster, 2015] Fuster, J. (2015). The Prefrontal Cortex. Academic Press, 5th edition.

[Gabbott et al., 2005] Gabbott, P. L., Warner, T. A., Jays, P. R., Salway, P., and Busby, S. J. (2005). Prefrontal cortex in the rat: projections to subcortical autonomic, motor, and limbic centers. Journal of Comparative Neurology, 492(2):145-177.

[Galán et al., 2015] Galán, F., Baker, M. R., Alter, K., and Baker, S. N. (2015). Degraded EEG decoding of wrist movements in absence of kinaesthetic feedback. Human brain mapping, 36(2):643-654.

[Gao and Hermes, 2015] Gao, X.-B. and Hermes, G. (2015). Neural plasticity in hypocretin neurons: the basis of hypocretinergic regulation of physiological and behavioral functions in animals. Frontiers in systems neuroscience, 9.

[Gauthier, 2017] Gauthier, I. (2017). The quest for the FFA led to the expertise account of its specialization. arXiv preprint arXiv:1702.07038.

[Gee et al., 2012] Gee, S., Ellwood, I., Patel, T., Luongo, F., Deisseroth, K., and Sohal, V. S. (2012). Synaptic activity unmasks dopamine D2 receptor modulation of a specific class of layer $\mathrm{V}$ pyramidal neurons in prefrontal cortex. The Journal of Neuroscience, 32(14):4959-4971.

[Georgescu et al., 2005] Georgescu, D., Sears, R. M., Hommel, J. D., Barrot, M., Bolanos, C. A., Marsh, D. J., Bednarek, M. A., Bibb, J. A., Maratos-Flier, E., Nestler, E. J., et al. (2005). The hypothalamic neuropeptide melanin-concentrating hormone acts in the nucleus accumbens to modulate feeding behavior and forced-swim performance. Journal of Neuroscience, 25(11):2933-2940.

[Gibson and Thomas, 1999] Gibson, E. and Thomas, J. (1999). Memory limitations and structural forgetting: The perception of complex ungrammatical sentences as grammatical. Language and Cognitive Processes, 14(3):225-248.

[Gimenes et al., 2009] Gimenes, M., Rigalleau, F., and Gaonac'h, D. (2009). The effect of noun phrase type on working memory saturation during sentence comprehension. European Journal of Cognitive Psychology, 21(7):980-1000.

[Giovannucci et al., 2017] Giovannucci, A., Badura, A., Deverett, B., Najafi, F., Pereira, T. D., Gao, Z., Ozden, I., Kloth, A. D., Pnevmatikakis, E., Paninski, L., et al. (2017). Cerebellar granule cells acquire a widespread predictive feedback signal during motor learning. Nature Neuroscience, 20(5):727-734.

[Goldberg, 2006] Goldberg, A. E. (2006). Constructions at work: The nature of generalization in language. Oxford University Press on Demand. 
[Goldman-Rakic et al., 1990] Goldman-Rakic, P., Lidow, M., and Gallager, D. (1990). Overlap of dopaminergic, adrenergic, and serotoninergic receptors and complementarity of their subtypes in primate prefrontal cortex. The Journal of Neuroscience, 10(7):21252138.

[Goll et al., 2015] Goll, Y., Atlan, G., and Citri, A. (2015). Attention: the claustrum. Trends in neurosciences, 38(8):486-495.

[Gonzales and Smith, 2015] Gonzales, K. K. and Smith, Y. (2015). Cholinergic interneurons in the dorsal and ventral striatum: anatomical and functional considerations in normal and diseased conditions. Annals of the New York Academy of Sciences.

[González et al., 2016a] González, J. A., Iordanidou, P., Strom, M., Adamantidis, A., and Burdakov, D. (2016a). Awake dynamics and brain-wide direct inputs of hypothalamic $\mathrm{MCH}$ and orexin networks. Nature communications, 7.

[González et al., 2016b] González, J. A., Jensen, L. T., Iordanidou, P., Strom, M., Fugger, L., and Burdakov, D. (2016b). Inhibitory interplay between orexin neurons and eating. Current Biology, 26(18):2486-2491.

[Govindaiah and Cox, 2006] Govindaiah, G. and Cox, C. L. (2006). Modulation of thalamic neuron excitability by orexins. Neuropharmacology, 51(3):414-425.

[Graeff, 2017] Graeff, F. G. (2017). Translational approach to the pathophysiology of panic disorder: Focus on serotonin and endogenous opioids. Neuroscience \& Biobehavioral Reviews.

[Gray et al., 2015] Gray, J. M., Vecchiarelli, H. A., Morena, M., Lee, T. T., Hermanson, D. J., Kim, A. B., McLaughlin, R. J., Hassan, K. I., Kühne, C., Wotjak, C. T., et al. (2015). Corticotropin-releasing hormone drives anandamide hydrolysis in the amygdala to promote anxiety. Journal of Neuroscience, 35(9):3879-3892.

[Graybiel and Mink, 2009] Graybiel, A. and Mink, J. (2009). The basal ganglia and cognition. In Gazzaniga, M., editor, The Cognitive Neurosciences IV. MIT Press, Cambridge, MA.

[Graziano, 2016] Graziano, M. S. (2016). Ethological action maps: a paradigm shift for the motor cortex. Trends in cognitive sciences, 20(2):121-132.

[Gregoriou et al., 2012] Gregoriou, G. G., Gotts, S. J., and Desimone, R. (2012). Celltype-specific synchronization of neural activity in FEF with V4 during attention. Neuron, 73(3):581-594.

[Grillner, 2014] Grillner, S. (2014). Megascience efforts and the brain. Neuron, 82(6):1209-1211.

[Groc et al., 2008] Groc, L., Choquet, D., and Chaouloff, F. (2008). The stress hormone corticosterone conditions AMPAR surface trafficking and synaptic potentiation. Nature neuroscience, 11(8):868-870.

[Grosprêtre et al., 2016] Grosprêtre, S., Ruffino, C., and Lebon, F. (2016). Motor imagery and cortico-spinal excitability: a review. European journal of sport science, 16(3):317324. 
[Guiard et al., 2008] Guiard, B. P., El Mansari, M., Merali, Z., and Blier, P. (2008). Functional interactions between dopamine, serotonin and norepinephrine neurons: an in-vivo electrophysiological study in rats with monoaminergic lesions. International Journal of Neuropsychopharmacology, 11(5):625-639.

[Guillot et al., 2014] Guillot, A., Di Rienzo, F., and Collet, C. (2014). The neurofunctional architecture of motor imagery. In Advanced Brain Neuroimaging Topics in Health and Disease-Methods and Applications. InTech.

[Guo et al., 2016] Guo, C., Witter, L., Rudolph, S., Elliott, H. L., Ennis, K. A., and Regehr, W. G. (2016). Purkinje cells directly inhibit granule cells in specialized regions of the cerebellar cortex. Neuron, 91(6):1330-1341.

[Guo et al., 2007] Guo, H.-T., Zhang, R.-H., Huang, L.-Y., Li, J., Liu, Y.-L., Bi, H., Zhang, Q. Y., Wang, Y.-M., Sun, X., Ma, X.-L., et al. (2007). Mechanisms involved in the hypotensive effect of a $\kappa$-opioid receptor agonist in hypertensive rats. Archives of medical research, 38(7):723-729.

[Guo et al., 2017] Guo, Z. V., Inagaki, H. K., Daie, K., Druckmann, S., Gerfen, C. R., and Svoboda, K. (2017). Maintenance of persistent activity in a frontal thalamocortical loop. Nature.

[Gutkowska et al., 2014] Gutkowska, J., Jankowski, M., and Antunes-Rodrigues, J. (2014). The role of oxytocin in cardiovascular regulation. Brazilian Journal of Medical and Biological Research, 47(3):206-214.

[Haas et al., 2008] Haas, H. L., Sergeeva, O. A., and Selbach, O. (2008). Histamine in the nervous system. Physiological reviews, 88(3):1183-1241.

[Hagena and Manahan-Vaughan, 2017] Hagena, H. and Manahan-Vaughan, D. (2017). The serotonergic 5-HT4 receptor: A unique modulator of hippocampal synaptic information processing and cognition. Neurobiology of learning and memory, 138:145-153.

[Hahamy et al., 2017] Hahamy, A., Macdonald, S. N., van den Heiligenberg, F., Kieliba, P., Emir, U., Malach, R., Johansen-Berg, H., Brugger, P., Culham, J. C., and Makin, T. R. (2017). Representation of multiple body parts in the missing-hand territory of congenital one-handers. Current Biology, 27(9):1350-1355.

[Haj-Dahmane, 2001] Haj-Dahmane, S. (2001). D2-like dopamine receptor activation excites rat dorsal raphe 5-HT neurons in vitro. European Journal of Neuroscience, 14(1):125-134.

[Haj-Dahmane and Shen, 2005] Haj-Dahmane, S. and Shen, R.-Y. (2005). The wakepromoting peptide orexin-B inhibits glutamatergic transmission to dorsal raphe nucleus serotonin neurons through retrograde endocannabinoid signaling. Journal of Neuroscience, 25(4):896-905.

[Halasz et al., 2009] Halasz, J., Zelena, D., Toth, M., Tulogdi, A., Mikics, E., and Haller, J. (2009). Substance P neurotransmission and violent aggression: the role of tachykinin NK 1 receptors in the hypothalamic attack area. European journal of pharmacology, 611(1):35-43. 
[Halgren et al., 2015] Halgren, E., Kaestner, E., Marinkovic, K., Cash, S. S., Wang, C., Schomer, D. L., Madsen, J. R., and Ulbert, I. (2015). Laminar profile of spontaneous and evoked theta: rhythmic modulation of cortical processing during word integration. Neuropsychologia, 76:108-124.

[Han et al., 2015] Han, S., Soleiman, M. T., Soden, M. E., Zweifel, L. S., and Palmiter, R. D. (2015). Elucidating an affective pain circuit that creates a threat memory. Cell, 162(2):363-374.

[Harari-Dahan and Bernstein, 2014] Harari-Dahan, O. and Bernstein, A. (2014). A general approach-avoidance hypothesis of oxytocin: accounting for social and non-social effects of oxytocin. Neuroscience \& Biobehavioral Reviews, 47:506-519.

[Harris and Shepherd, 2015] Harris, K. D. and Shepherd, G. M. (2015). The neocortical circuit: themes and variations. Nature neuroscience, 18(2):170-181.

[Hart et al., 2013] Hart, J., Maguire, M. J., Motes, M., Mudar, R. A., Chiang, H.-S., Womack, K. B., and Kraut, M. A. (2013). Semantic memory retrieval circuit: Role of preSMA, caudate, and thalamus. Brain and language, 126(1):89-98.

[Haubensak et al., 2010] Haubensak, W., Kunwar, P. S., Cai, H., Ciocchi, S., Wall, N. R., Ponnusamy, R., Biag, J., Dong, H.-W., Deisseroth, K., Callaway, E. M., et al. (2010). Genetic dissection of an amygdala microcircuit that gates conditioned fear. Nature, 468(7321):270-276.

[Hayama et al., 2013] Hayama, T., Noguchi, J., Watanabe, S., Takahashi, N., HayashiTakagi, A., Ellis-Davies, G. C., Matsuzaki, M., and Kasai, H. (2013). GABA promotes the competitive selection of dendritic spines by controlling local $\mathrm{Ca} 2+$ signaling. Nature neuroscience, 16(10):1409-1416.

[He et al., 2016] He, C., Luo, F., Chen, X., Chen, F., Li, C., Ren, S., Qiao, Q., Zhang, J., de Lecea, L., Gao, D., et al. (2016). Superficial layer-specific histaminergic modulation of medial entorhinal cortex required for spatial learning. Cerebral Cortex, 26(4):15901608.

[Hegeman et al., 2016] Hegeman, D. J., Hong, E. S., Hernández, V. M., and Chan, S. C. (2016). The external globus pallidus: Progress and perspectives. European Journal of Neuroscience.

[Heimler et al., 2015] Heimler, B., Striem-Amit, E., and Amedi, A. (2015). Origins of task-specific sensory-independent organization in the visual and auditory brain: neuroscience evidence, open questions and clinical implications. Current opinion in neurobiology, 35:169-177.

[Heiney et al., 2014] Heiney, S. A., Kim, J., Augustine, G. J., and Medina, J. F. (2014). Precise control of movement kinematics by optogenetic inhibition of Purkinje cell activity. The Journal of Neuroscience, 34(6):2321-2330.

[Henckens et al., 2017] Henckens, M., Printz, Y., Shamgar, U., Dine, J., Lebow, M., Drori, Y., Kuehne, C., Kolarz, A., Eder, M., Deussing, J., et al. (2017). CRF receptor type 2 neurons in the posterior bed nucleus of the stria terminalis critically contribute to stress recovery. Molecular psychiatry, 22(12):1691. 
[Henzl, 2013] Henzl, M. T. (2013). Parvalbumin. Encyclopedia of Metalloproteins, pages 1669-1678.

[Hestrin and Galarreta, 2005] Hestrin, S. and Galarreta, M. (2005). Electrical synapses define networks of neocortical GABAergic neurons. Trends in neurosciences, 28(6):304309.

[Hétu et al., 2013] Hétu, S., Grégoire, M., Saimpont, A., Coll, M.-P., Eugène, F., Michon, P.-E., and Jackson, P. L. (2013). The neural network of motor imagery: an ALE metaanalysis. Neuroscience \& Biobehavioral Reviews, 37(5):930-949.

[Hickok and Small, 2015] Hickok, G. and Small, S. L. (2015). Neurobiology of language. Academic Press.

[Hikosaka, 2007] Hikosaka, O. (2007). GABAergic output of the basal ganglia. Progress in brain research, 160:209-226.

[Hill et al., 2007] Hill, E. L., Gallopin, T., Férézou, I., Cauli, B., Rossier, J., Schweitzer, P., and Lambolez, B. (2007). Functional CB1 receptors are broadly expressed in neocortical GABAergic and glutamatergic neurons. Journal of neurophysiology, 97(4):2580-2589.

[Hilscher et al., 2017] Hilscher, M. M., Leão, R. N., Edwards, S. J., Leão, K. E., and Kullander, K. (2017). Chrna2-Martinotti cells synchronize layer 5 type A pyramidal cells via rebound excitation. PLoS biology, 15(2):e2001392.

[Hoffstaedter et al., 2014] Hoffstaedter, F., Grefkes, C., Caspers, S., Roski, C., PalomeroGallagher, N., Laird, A. R., Fox, P. T., and Eickhoff, S. B. (2014). The role of anterior midcingulate cortex in cognitive motor control. Human brain mapping, 35(6):27412753.

[Hommel, 2009] Hommel, B. (2009). Action control according to TEC (theory of event coding). Psychological Research PRPF, 73(4):512-526.

[Horton and Adams, 2005] Horton, J. C. and Adams, D. L. (2005). The cortical column: a structure without a function. Philosophical Transactions of the Royal Society of London B: Biological Sciences, 360(1456):837-862.

[Hughes and Crunelli, 2007] Hughes, S. W. and Crunelli, V. (2007). Just a phase they're going through: The complex interaction of intrinsic high-threshold bursting and gap junctions in the generation of thalamic $\alpha$ and $\theta$ rhythms. International Journal of Psychophysiology, 64(1):3-17.

[Ishikawa et al., 2014] Ishikawa, T., Tomatsu, S., Tsunoda, Y., Lee, J., Hoffman, D. S., and Kakei, S. (2014). Releasing dentate nucleus cells from Purkinje cell inhibition generates output from the cerebrocerebellum. PloS one, 9(10):e108774.

[Iyengar et al., 2017] Iyengar, S., Ossipov, M. H., and Johnson, K. W. (2017). The role of calcitonin gene-related peptide in peripheral and central pain mechanisms including migraine. Pain, 158(4):543.

[Jackendoff, 2002] Jackendoff, R. (2002). Foundation of language-brain, meaning, grammar, evolution. Oxford: Oxford university press. 
[Jacobsen et al., 2006] Jacobsen, K., Höistad, M., Staines, W., and Fuxe, K. (2006). The distribution of dopamine D1 receptor and $\mu$-opioid receptor 1 receptor immunoreactivities in the amygdala and interstitial nucleus of the posterior limb of the anterior commissure: Relationships to tyrosine hydroxylase and opioid peptide terminal systems. Neuroscience, 141(4):2007-2018.

[Jahanshahi et al., 1995] Jahanshahi, M., Jenkins, I. H., Brown, R. G., Marsden, C. D., Passingham, R. E., and Brooks, D. J. (1995). Self-initiated versus externally triggered movements: I. an investigation using measurement of regional cerebral blood flow with PET and movement-related potentials in normal and Parkinson's disease subjects. Brain, 118(4):913-933.

[Jakab and Goldman-Rakic, 2000] Jakab, R. L. and Goldman-Rakic, P. S. (2000). Segregation of serotonin 5-HT2A and 5-HT3 receptors in inhibitory circuits of the primate cerebral cortex. Journal of Comparative Neurology, 417(3):337-348.

[Jeannerod, 1995] Jeannerod, M. (1995). Mental imagery in the motor context. Neuropsychologia, 33(11):1419-1432.

[Jhou et al., 2009] Jhou, T. C., Geisler, S., Marinelli, M., Degarmo, B. A., and Zahm, D. S. (2009). The mesopontine rostromedial tegmental nucleus: a structure targeted by the lateral habenula that projects to the ventral tegmental area of Tsai and substantia nigra compacta. Journal of Comparative Neurology, 513(6):566-596.

[Jiang et al., 2013] Jiang, X., Wang, G., Lee, A. J., Stornetta, R. L., and Zhu, J. J. (2013). The organization of two new cortical interneuronal circuits. Nature neuroscience, 16(2):210-218.

[Joëls et al., 2012] Joëls, M., Sarabdjitsingh, R. A., and Karst, H. (2012). Unraveling the time domains of corticosteroid hormone influences on brain activity: rapid, slow, and chronic modes. Pharmacological reviews, 64(4):901-938.

[Jörntell, 2017] Jörntell, H. (2017). Cerebellar physiology: links between microcircuitry properties and sensorimotor functions. The Journal of physiology, 595(1):11-27.

[Joshi et al., 2016] Joshi, A., Kalappa, B. I., Anderson, C. T., and Tzounopoulos, T. (2016). Cell-specific cholinergic modulation of excitability of layer $5 \mathrm{~b}$ principal neurons in mouse auditory cortex. Journal of Neuroscience, 36(32):8487-8499.

[Jüngling et al., 2015] Jüngling, K., Lange, M. D., Szkudlarek, H. J., Lesting, J., Erdmann, F. S., Doengi, M., Kügler, S., and Pape, H.-C. (2015). Increased gabaergic efficacy of central amygdala projections to neuropeptide $S$ neurons in the brainstem during fear memory retrieval. Neuropsychopharmacology, 40(12):2753-2763.

[Kaas, 2013] Kaas, J. H. (2013). The evolution of brains from early mammals to humans. Wiley Interdisciplinary Reviews: Cognitive Science, 4(1):33-45.

[Kafkas and Montaldi, 2015] Kafkas, A. and Montaldi, D. (2015). The pupillary response discriminates between subjective and objective familiarity and novelty. Psychophysiology, 52(10):1305-1316.

[Kahneman, 2011] Kahneman, D. (2011). Thinking, fast and slow. Macmillan. 
[Kamin and Kertes, 2017] Kamin, H. S. and Kertes, D. A. (2017). Cortisol and DHEA in development and psychopathology. Hormones and behavior, 89:69-85.

[Kaneko, 2013] Kaneko, T. (2013). Local connections of excitatory neurons in motorassociated cortical areas of the rat. Frontiers in neural circuits, 7.

[Karimi and Ferreira, 2016] Karimi, H. and Ferreira, F. (2016). Good-enough linguistic representations and online cognitive equilibrium in language processing. The Quarterly Journal of Experimental Psychology, 69(5):1013-1040.

[Karnani et al., 2016] Karnani, M. M., Jackson, J., Ayzenshtat, I., Sichani, A. H., Manoocheri, K., Kim, S., and Yuste, R. (2016). Opening holes in the blanket of inhibition: localized lateral disinhibition by VIP interneurons. Journal of Neuroscience, 36(12):3471-3480.

[Karst et al., 2010] Karst, H., Berger, S., Erdmann, G., Schütz, G., and Joëls, M. (2010). Metaplasticity of amygdalar responses to the stress hormone corticosterone. Proceedings of the National Academy of Sciences, 107(32):14449-14454.

[Kash et al., 2015] Kash, T. L., Pleil, K. E., Marcinkiewcz, C. A., Lowery-Gionta, E. G., Crowley, N., Mazzone, C., Sugam, J., Hardaway, J. A., and McElligott, Z. A. (2015). Neuropeptide regulation of signaling and behavior in the BNST. Molecules and cells, 38(1):1.

[Katsuura and Taha, 2010] Katsuura, Y. and Taha, S. A. (2010). Modulation of feeding and locomotion through mu and delta opioid receptor signaling in the nucleus accumbens. Neuropeptides, 44(3):225-232.

[Kawaguchi, 2017] Kawaguchi, Y. (2017). Pyramidal cell subtypes and their synaptic connections in layer 5 of rat frontal cortex. Cerebral Cortex, 27(12):5755-5771.

[Kawahara et al., 2006] Kawahara, J.-I., Kumada, T., and Di Lollo, V. (2006). The attentional blink is governed by a temporary loss of control. Psychonomic Bulletin \& Review, 13(5):886-890.

[Kawashima, 2017] Kawashima, T. (2017). The role of the serotonergic system in motor control. Neuroscience Research.

[Kela et al., 2003] Kela, J., Salmi, P., Rimondini-Giorgini, R., Heilig, M., and Wahlestedt, C. (2003). Behavioural analysis of melanin-concentrating hormone in rats: evidence for orexigenic and anxiolytic properties. Regulatory peptides, 114(2):109-114.

[Kelley et al., 2002] Kelley, A., Bakshi, V., Haber, S., Steininger, T., Will, M., and Zhang, M. (2002). Opioid modulation of taste hedonics within the ventral striatum. Physiology \& behavior, 76(3):365-377.

[Ketchesin et al., 2017] Ketchesin, K. D., Huang, N. S., and Seasholtz, A. F. (2017). Cell type-specific expression of corticotropin-releasing hormone-binding protein in GABAergic interneurons in the prefrontal cortex. Frontiers in neuroanatomy, 11:90.

[Kikuchi et al., 2012] Kikuchi, T., Matsumoto, R., Mikuni, N., Yokoyama, Y., Matsumoto, A., Ikeda, A., Fukuyama, H., Miyamoto, S., and Hashimoto, N. (2012). Asymmetric bilateral effect of the supplementary motor area proper in the human motor system. Clinical neurophysiology, 123(2):324-334. 
[Kikyo et al., 2002] Kikyo, H., Ohki, K., and Miyashita, Y. (2002). Neural correlates for feeling-of-knowing: an fMRI parametric analysis. Neuron, 36(1):177-186.

[Kilner et al., 2004] Kilner, J. M., Vargas, C., Duval, S., Blakemore, S.-J., and Sirigu, A. (2004). Motor activation prior to observation of a predicted movement. Nature neuroscience, 7(12):1299.

[Kim et al., 2017a] Kim, J., Kim, Y., Nakajima, R., Shin, A., Jeong, M., Park, A. H., Jeong, Y., Jo, S., Yang, S., Park, H., et al. (2017a). Inhibitory basal ganglia inputs induce excitatory motor signals in the thalamus. Neuron, 95(5):1181-1196.

[Kim et al., 2016] Kim, J., Matney, C. J., Roth, R. H., and Brown, S. P. (2016). Synaptic organization of the neuronal circuits of the claustrum. Journal of Neuroscience, 36(3):773-784.

[Kim et al., 2017b] Kim, J., Zhang, X., Muralidhar, S., LeBlanc, S. A., and Tonegawa, S. (2017b). Basolateral to central amygdala neural circuits for appetitive behaviors. Neuron, 93(6):1464-1479.

[Kitamura et al., 2015] Kitamura, T., Sun, C., Martin, J., Kitch, L. J., Schnitzer, M. J., and Tonegawa, S. (2015). Entorhinal cortical ocean cells encode specific contexts and drive context-specific fear memory. Neuron, 87(6):1317-1331.

[Kitanishi and Matsuo, 2017] Kitanishi, T. and Matsuo, N. (2017). Organization of the claustrum-to-entorhinal cortical connection in mice. Journal of Neuroscience, 37(2):269-280.

[Kjaerby et al., 2016] Kjaerby, C., Athilingam, J., Robinson, S. E., Iafrati, J., and Sohal, V. S. (2016). Serotonin 1B receptors regulate prefrontal function by gating callosal and hippocampal inputs. Cell Reports, 17(11):2882-2890.

[Klomp et al., 2014] Klomp, A., Hamelink, R., Feenstra, M., Denys, D., and Reneman, L. (2014). Increased response to a 5-HT challenge after discontinuation of chronic serotonin uptake inhibition in the adult and adolescent rat brain. PloS one, 9(6):e99873.

[Knudsen et al., 2012] Knudsen, E. B., Flint III, R. D., and Moxon, K. A. (2012). Encoding of temporal intervals in the rat hindlimb sensorimotor cortex. Frontiers in systems neuroscience, 6:67.

[Koch, 2004] Koch, C. (2004). The quest for consciousness: A neuroscientific approach. Roberts \& Co.

[Koch and Marcus, 2014] Koch, C. and Marcus, G. (2014). Neuroscience in 2064: a look at the last century. In Marcus, G. and Freeman, J., editors, The Future of the Brain: Essays by the World's Leading Neuroscientists. Princeton University Press.

[Kopell et al., 2010] Kopell, N., Kramer, M. A., Malerba, P., and Whittington, M. A. (2010). Are different rhythms good for different functions? Frontiers in human neuroscience, 4 .

[Kotchoubey, 2006] Kotchoubey, B. (2006). Event-related potentials, cognition, and behavior: a biological approach. Neuroscience \& Biobehavioral Reviews, 30(1):42-65. 
[Koubeissi et al., 2014] Koubeissi, M. Z., Bartolomei, F., Beltagy, A., and Picard, F. (2014). Electrical stimulation of a small brain area reversibly disrupts consciousness. Epilepsy \& Behavior, 37:32-35.

[Koutsikou et al., 2014] Koutsikou, S., Crook, J. J., Earl, E. V., Leith, J. L., Watson, T. C., Lumb, B. M., and Apps, R. (2014). Neural substrates underlying fear-evoked freezing: the periaqueductal grey-cerebellar link. The Journal of physiology, 592(10):2197-2213.

[Kozyrev and Coolen, 2015] Kozyrev, N. and Coolen, L. M. (2015). Activation of mu or delta opioid receptors in the lumbosacral spinal cord is essential for ejaculatory reflexes in male rats. PloS one, 10(3): $\mathrm{e} 0121130$.

[Krauzlis et al., 2013] Krauzlis, R., Lovejoy, L., and Zénon, A. (2013). Superior colliculus and visual spatial attention. Annual review of neuroscience, 36:165-182.

[Kupchik et al., 2015] Kupchik, Y. M., Brown, R. M., Heinsbroek, J. A., Lobo, M. K., Schwartz, D. J., and Kalivas, P. W. (2015). Coding the direct/indirect pathways by D1 and D2 receptors is not valid for accumbens projections. Nature neuroscience.

[LaBerge, 2002] LaBerge, D. (2002). Attentional control: brief and prolonged. Psychological research, 66(4):220-233.

[Langacker, 2008] Langacker, R. W. (2008). Cognitive grammar: A basic introduction. OUP USA.

[Lazarov et al., 2011] Lazarov, N. E., Usunoff, K. G., Schmitt, O., Itzev, D. E., Rolfs, A., and Wree, A. (2011). Amygdalotrigeminal projection in the rat: an anterograde tracing study. Annals of Anatomy-Anatomischer Anzeiger, 193(2):118-126.

[Lean et al., 2018] Lean, G. A., Liu, Y.-j., and Lyon, D. C. (2018). Cell type specific tracing of the subcortical input to primary visual cortex from the basal forebrain. Journal of Comparative Neurology.

[Lecci et al., 2000] Lecci, A., Giuliani, S., Tramontana, M., Meini, S., Santicioli, P., and Maggi, C. A. (2000). Tachykinin-mediated effect of nociceptin in the rat urinary bladder in vivo. European journal of pharmacology, 389(1):99-102.

[LeDoux, 2015] LeDoux, J. (2015). Anxious. Oneworld Publications.

[Lee et al., 2011] Lee, C., Parks, G. S., and Civelli, O. (2011). Anxiolytic effects of the MCH1R antagonist TPI 1361-17. Journal of Molecular Neuroscience, 43(2):132-137.

[Lerner et al., 2015] Lerner, T. N., Shilyansky, C., Davidson, T. J., Evans, K. E., Beier, K. T., Zalocusky, K. A., Crow, A. K., Malenka, R. C., Luo, L., Tomer, R., et al. (2015). Intact-brain analyses reveal distinct information carried by SNc dopamine subcircuits. Cell, 162(3):635-647.

[Letzkus et al., 2015] Letzkus, J. J., Wolff, S. B., and Lüthi, A. (2015). Disinhibition, a circuit mechanism for associative learning and memory. Neuron, 88(2):264-276.

[Letzkus et al., 2011] Letzkus, J. J., Wolff, S. B., Meyer, E. M., Tovote, P., Courtin, J., Herry, C., and Lüthi, A. (2011). A disinhibitory microcircuit for associative fear learning in the auditory cortex. Nature, 480(7377):331-335. 
[Li et al., 2017a] Li, H., Jia, X., Qi, Z., Fan, X., Ma, T., Ni, H., Li, C.-s. R., and Li, K. (2017a). Altered functional connectivity of the basal nucleus of Meynert in mild cognitive impairment: A resting-state fMRI study. Frontiers in aging neuroscience, 9.

[Li et al., 2009] Li, L., Du, Y., Li, N., Wu, X., and Wu, Y. (2009). Top-down modulation of prepulse inhibition of the startle reflex in humans and rats. Neuroscience \& Biobehavioral Reviews, 33(8):1157-1167.

[Li et al., 2012] Li, Q., Luo, T., Jiang, X., and Wang, J. (2012). Anxiolytic effects of 5HT 1A receptors and anxiogenic effects of 5-HT 2C receptors in the amygdala of mice. Neuropharmacology, 62(1):474-484.

[Li et al., 2017b] Li, X., Zhang, Y., Xia, J., and Swaab, T. (2017b). Internal mechanisms underlying anticipatory language processing: Evidence from event-related-potentials and neural oscillations. Neuropsychologia, 102:70-81.

[Li and van den Pol, 2008] Li, Y. and van den Pol, A. N. (2008). $\mu$-opioid receptormediated depression of the hypothalamic hypocretin/orexin arousal system. The Journal of neuroscience, 28(11):2814-2819.

[Liang et al., 2012] Liang, M., Mouraux, A., and Iannetti, G. D. (2012). Bypassing primary sensory cortices - a direct thalamocortical pathway for transmitting salient sensory information. Cerebral Cortex, page bhr363.

[Lichtenberg and Wassum, 2017] Lichtenberg, N. T. and Wassum, K. M. (2017). Amygdala mu-opioid receptors mediate the motivating influence of cue-triggered reward expectations. European Journal of Neuroscience, 121(3):381-387.

[Lima et al., 2016] Lima, C. F., Krishnan, S., and Scott, S. K. (2016). Roles of supplementary motor areas in auditory processing and auditory imagery. Trends in neurosciences, 39(8):527-542.

[Lin et al., 2015] Lin, W.-J., Horner, A. J., Bisby, J. A., and Burgess, N. (2015). Medial prefrontal cortex: Adding value to imagined scenarios. Journal of cognitive neuroscience, 27(10):1957.

[Lindstrom et al., 2010] Lindstrom, S. H., Azizi, N., Weller, C., and Wilson, M. (2010). Retinal input to efferent target amacrine cells in the avian retina. Visual neuroscience, 27(3-4):103-118.

[Liu et al., 2014] Liu, Z., Zhou, J., Li, Y., Hu, F., Lu, Y., Ma, M., Feng, Q., Zhang, J.-e., Wang, D., Zeng, J., et al. (2014). Dorsal raphe neurons signal reward through 5-HT and glutamate. Neuron, 81(6):1360-1374.

[Lu et al., 2013] Lu, Z.-h., Fukuda, S., Minakawa, Y., Yasuda, A., Sakamoto, H., Sawamura, S., Takahashi, H., and Ishii, N. (2013). Melanin concentrating hormone induces hippocampal acetylcholine release via the medial septum in rats. Peptides, 44:32-39.

[Luppi et al., 2017] Luppi, P.-H., Billwiller, F., and Fort, P. (2017). Selective activation of a few limbic structures during paradoxical (REM) sleep by the claustrum and the supramammillary nucleus: evidence and function. Current Opinion in Neurobiology, 44:59-64. 
[Machado et al., 2013] Machado, S., Arias-Carrión, O., Paes, F., Ribeiro, P., Cagy, M., Piedade, R., Almada, L. F., Anghinah, R., Basile, L., Moro, M. F., et al. (2013). Changes in cortical activity during real and imagined movements: an ERP study. Clinical practice and epidemiology in mental health: $C P \& E M H, 9: 196$.

[Maejima et al., 2014] Maejima, Y., Sakuma, K., Santoso, P., Gantulga, D., Katsurada, K., Ueta, Y., Hiraoka, Y., Nishimori, K., Tanaka, S., Shimomura, K., et al. (2014). Oxytocinergic circuit from paraventricular and supraoptic nuclei to arcuate POMC neurons in hypothalamus. FEBS letters, 588(23):4404-4412.

[Mahadevan and Woodin, 2016] Mahadevan, V. and Woodin, M. A. (2016). Regulation of neuronal chloride homeostasis by neuromodulators. The Journal of physiology, 594(10):2593-2605.

[Maier et al., 2002] Maier, M., Armand, J., Kirkwood, P., Yang, H.-W., Davis, J., and Lemon, R. (2002). Differences in the corticospinal projection from primary motor cortex and supplementary motor area to macaque upper limb motoneurons: an anatomical and electrophysiological study. Cerebral Cortex, 12(3):281-296.

[Maier, 2015] Maier, S. F. (2015). Behavioral control blunts reactions to contemporaneous and future adverse events: medial prefrontal cortex plasticity and a corticostriatal network. Neurobiology of stress, 1:12-22.

[Major et al., 2013] Major, G., Larkum, M. E., and Schiller, J. (2013). Active properties of neocortical pyramidal neuron dendrites. Annual review of neuroscience, 36:1-24.

[Malhotra et al., 2012] Malhotra, S., Cross, R. W., and van der Meer, M. A. (2012). Theta phase precession beyond the hippocampus. Reviews in the neurosciences, 23(1):39-65.

[Maranesi et al., 2014] Maranesi, M., Livi, A., Fogassi, L., Rizzolatti, G., and Bonini, L. (2014). Mirror neuron activation prior to action observation in a predictable context. Journal of Neuroscience, 34(45):14827-14832.

[Marcinkiewcz et al., 2016] Marcinkiewcz, C. A., Mazzone, C. M., D’Agostino, G., Halladay, L. R., Hardaway, J. A., DiBerto, J. F., Navarro, M., Burnham, N., Cristiano, C., Dorrier, C. E., et al. (2016). Serotonin engages an anxiety and fear-promoting circuit in the extended amygdala. Nature, 537(7618):97-101.

[Maren and Holmes, 2016] Maren, S. and Holmes, A. (2016). Stress and fear extinction. Neuropsychopharmacology, 41(1):58-79.

[Mariën et al., 2014] Mariën, P., Ackermann, H., Adamaszek, M., Barwood, C. H., Beaton, A., Desmond, J., De Witte, E., Fawcett, A. J., Hertrich, I., Küper, M., et al. (2014). Consensus paper: language and the cerebellum: an ongoing enigma. The Cerebellum, 13(3):386-410.

[Markov et al., 2014] Markov, N. T., Vezoli, J., Chameau, P., Falchier, A., Quilodran, R., Huissoud, C., Lamy, C., Misery, P., Giroud, P., Ullman, S., et al. (2014). Anatomy of hierarchy: feedforward and feedback pathways in macaque visual cortex. Journal of Comparative Neurology, 522(1):225-259.

[Marlin et al., 2015] Marlin, B. J., Mitre, M., D'amour, J. A., Chao, M. V., and Froemke, R. C. (2015). Oxytocin enables maternal behaviour by balancing cortical inhibition. Nature, 520(7548):499-504. 
[Marty and Llano, 2005] Marty, A. and Llano, I. (2005). Excitatory effects of GABA in established brain networks. Trends in neurosciences, 28(6):284-289.

[Matsumoto et al., 2001] Matsumoto, N., Minamimoto, T., Graybiel, A. M., and Kimura, M. (2001). Neurons in the thalamic CM-Pf complex supply striatal neurons with information about behaviorally significant sensory events. Journal of Neurophysiology, 85(2):960-976.

[Matyas et al., 2010] Matyas, F., Sreenivasan, V., Marbach, F., Wacongne, C., Barsy, B., Mateo, C., Aronoff, R., and Petersen, C. C. (2010). Motor control by sensory cortex. Science, 330(6008):1240-1243.

[McDevitt et al., 2014] McDevitt, R. A., Tiran-Cappello, A., Shen, H., Balderas, I., Britt, J. P., Marino, R. A., Chung, S. L., Richie, C. T., Harvey, B. K., and Bonci, A. (2014). Serotonergic versus nonserotonergic dorsal raphe projection neurons: differential participation in reward circuitry. Cell reports, 8(6):1857-1869.

[McDonald et al., 2011] McDonald, A., Muller, J., and Mascagni, F. (2011). Postsynaptic targets of GABAergic basal forebrain projections to the basolateral amygdala. Neuroscience, 183:144-159.

[McEwen et al., 2016] McEwen, B. S., Nasca, C., and Gray, J. D. (2016). Stress effects on neuronal structure: hippocampus, amygdala, and prefrontal cortex. Neuropsychopharmacology, 41(1):3-23.

[McGlone et al., 2014] McGlone, F., Wessberg, J., and Olausson, H. (2014). Discriminative and affective touch: sensing and feeling. Neuron, 82(4):737-755.

[McGuigan and Dollins, 1989] McGuigan, F. and Dollins, A. B. (1989). Patterns of covert speech behavior and phonetic coding. The Pavlovian journal of biological science, 24(1):19-26.

[Mechoulam and Parker, 2013] Mechoulam, R. and Parker, L. A. (2013). The endocannabinoid system and the brain. Annual review of psychology, 64:21-47.

[Mena-Segovia, 2016] Mena-Segovia, J. (2016). Structural and functional considerations of the cholinergic brainstem. Journal of Neural Transmission, 123(7):731-736.

[Mercer et al., 2013] Mercer, A. J., Hentges, S. T., Meshul, C. K., and Low, M. J. (2013). Unraveling the central proopiomelanocortin neural circuits. Frontiers in Neuroscience, 7.

[Mesulam, 2013] Mesulam, M.-M. (2013). Cholinergic circuitry of the human nucleus basalis and its fate in Alzheimer's disease. Journal of Comparative Neurology, 521(18):4124-4144.

[Micevych et al., 2017] Micevych, P. E., Mermelstein, P. G., and Sinchak, K. (2017). Estradiol membrane-initiated signaling in the brain mediates reproduction. Trends in Neurosciences.

[Milner and Goodale, 2008] Milner, A. D. and Goodale, M. A. (2008). Two visual systems re-viewed. Neuropsychologia, 46(3):774-785. 
[Miyazaki et al., 2011] Miyazaki, K., Miyazaki, K. W., and Doya, K. (2011). Activation of dorsal raphe serotonin neurons underlies waiting for delayed rewards. Journal of Neuroscience, 31(2):469-479.

[Mizushige et al., 2009] Mizushige, T., Saitoh, K., Manabe, Y., Nishizuka, T., Taka, Y., Eguchi, A., Yoneda, T., Matsumura, S., Tsuzuki, S., Inoue, K., et al. (2009). Preference for dietary fat induced by release of beta-endorphin in rats. Life sciences, 84(21):760765.

[Mizutani et al., 2017] Mizutani, K., Takahashi, S., Okamoto, S., Karube, F., and Fujiyama, F. (2017). Substance P effects exclusively on prototypic neurons in mouse globus pallidus. Brain Structure and Function, pages 1-22.

[Modirrousta and Fellows, 2008] Modirrousta, M. and Fellows, L. K. (2008). Medial prefrontal cortex plays a critical and selective role in 'feeling of knowing' meta-memory judgments. Neuropsychologia, 46(12):2958-2965.

[Morris et al., 2016] Morris, L. S., Kundu, P., Baek, K., Irvine, M. A., Mechelmans, D. J., Wood, J., Harrison, N. A., Robbins, T. W., Bullmore, E. T., and Voon, V. (2016). Jumping the gun: mapping neural correlates of waiting impulsivity and relevance across alcohol misuse. Biological psychiatry, 79(6):499-507.

[Morton et al., 2014] Morton, G. J., Meek, T. H., and Schwartz, M. W. (2014). Neurobiology of food intake in health and disease. Nature Reviews Neuroscience, 15(6):367-378.

[Moscovitch et al., 2016] Moscovitch, M., Cabeza, R., Winocur, G., and Nadel, L. (2016). Episodic memory and beyond: The hippocampus and neocortex in transformation. Annual review of psychology, 67:105-134.

[Moser et al., 2014] Moser, E. I., Roudi, Y., Witter, M. P., Kentros, C., Bonhoeffer, T., and Moser, M.-B. (2014). Grid cells and cortical representation. Nature Reviews Neuroscience, 15(7):466-481.

[Mountcastle, 1997] Mountcastle, V. B. (1997). The columnar organization of the neocortex. Brain, 120(4):701-722.

[Muñoz and Rudy, 2014] Muñoz, W. and Rudy, B. (2014). Spatiotemporal specificity in cholinergic control of neocortical function. Current opinion in neurobiology, 26:149160.

[Muñoz et al., 2017] Muñoz, W., Tremblay, R., Levenstein, D., and Rudy, B. (2017). Layer-specific modulation of neocortical dendritic inhibition during active wakefulness. Science, 355(6328):954-959.

[Myers et al., 2014] Myers, B., McKlveen, J. M., and Herman, J. P. (2014). Glucocorticoid actions on synapses, circuits, and behavior: implications for the energetics of stress. Frontiers in neuroendocrinology, 35(2):180-196.

[Nachev et al., 2008] Nachev, P., Kennard, C., and Husain, M. (2008). Functional role of the supplementary and pre-supplementary motor areas. Nature Reviews Neuroscience, 9(11):856-869. 
[Nalborczyk et al., 2017] Nalborczyk, L., Perrone-Bertolotti, M., Baeyens, C., Grandchamp, R., Polosan, M., Spinelli, E., Koster, E. H., and Lœvenbruck, H. (2017). Orofacial electromyographic correlates of induced verbal rumination. Biological Psychology, 127:53-63.

[Nelson and Chiavegatto, 2001] Nelson, R. J. and Chiavegatto, S. (2001). Molecular basis of aggression. Trends in Neurosciences, 24(12):713-719.

[Ninan, 2011] Ninan, I. (2011). Oxytocin suppresses basal glutamatergic transmission but facilitates activity-dependent synaptic potentiation in the medial prefrontal cortex. Journal of neurochemistry, 119(2):324-331.

[Niu et al., 2012] Niu, J.-G., Yokota, S., Tsumori, T., Oka, T., and Yasui, Y. (2012). Projections from the anterior basomedial and anterior cortical amygdaloid nuclei to melaninconcentrating hormone-containing neurons in the lateral hypothalamus of the rat. Brain research, 1479:31-43.

[Niziolek et al., 2013] Niziolek, C. A., Nagarajan, S. S., and Houde, J. F. (2013). What does motor efference copy represent? evidence from speech production. Journal of Neuroscience, 33(41):16110-16116.

[Okabe et al., 2015] Okabe, S., Yoshida, M., Takayanagi, Y., and Onaka, T. (2015). Activation of hypothalamic oxytocin neurons following tactile stimuli in rats. Neuroscience letters, 600:22-27.

[Olds, 2016] Olds, J. L. (2016). The rise of team neuroscience. Nature Reviews Neuroscience.

[Overton et al., 2014] Overton, P., Vautrelle, N., and Redgrave, P. (2014). Sensory regulation of dopaminergic cell activity: phenomenology, circuitry and function. Neuroscience, 282:1-12.

[Palomares-Castillo et al., 2012] Palomares-Castillo, E., Hernández-Pérez, O. R., PérezCarrera, D., Crespo-Ramírez, M., Fuxe, K., and de la Mora, M. P. (2012). The intercalated paracapsular islands as a module for integration of signals regulating anxiety in the amygdala. Brain research, 1476:211-234.

[Pan and Sakagami, 2012] Pan, X. and Sakagami, M. (2012). Category representation and generalization in the prefrontal cortex. European Journal of Neuroscience, 35(7):10831091.

[Pape and Pare, 2010] Pape, H.-C. and Pare, D. (2010). Plastic synaptic networks of the amygdala for the acquisition, expression, and extinction of conditioned fear. Physiological reviews, 90(2):419-463.

[Paredes, 2014] Paredes, R. (2014). Opioids and sexual reward. Pharmacology Biochemistry and Behavior, 121:124-131.

[Paul, 2013] Paul, W. (2013). Fundamental immunology, 7th edition.

[Pearson et al., 2015] Pearson, J., Naselaris, T., Holmes, E. A., and Kosslyn, S. M. (2015). Mental imagery: functional mechanisms and clinical applications. Trends in cognitive sciences, 19(10):590-602. 
[Perez et al., 2012] Perez, V. B., Vogel, E. K., Luck, S., and Kappenman, E. (2012). What ERPs can tell us about working memory. The Oxford handbook of event-related potential components, pages 361-372.

[Peris et al., 2017] Peris, J., MacFadyen, K., Smith, J. A., de Kloet, A. D., Wang, L., and Krause, E. G. (2017). Oxytocin receptors are expressed on dopamine and glutamate neurons in the mouse ventral tegmental area that project to nucleus accumbens and other mesolimbic targets. Journal of Comparative Neurology.

[Perrier and Cotel, 2015] Perrier, J.-F. and Cotel, F. (2015). Serotonergic modulation of spinal motor control. Current opinion in neurobiology, 33:1-7.

[Pfeiffer and Foster, 2013] Pfeiffer, B. E. and Foster, D. J. (2013). Hippocampal place-cell sequences depict future paths to remembered goals. Nature, 497(7447):74-79.

[Pickering and Garrod, 2013] Pickering, M. J. and Garrod, S. (2013). An integrated theory of language production and comprehension. Behavioral and Brain Sciences, 36(4):329347.

[Pinard et al., 2012] Pinard, C. R., Mascagni, F., and McDonald, A. J. (2012). Medial prefrontal cortical innervation of the intercalated nuclear region of the amygdala. Neuroscience, 205:112-124.

[Pinker and Jackendoff, 2005] Pinker, S. and Jackendoff, R. (2005). The faculty of language: what's special about it? Cognition, 95(2):201-236.

[Poorthuis et al., 2013] Poorthuis, R. B., Bloem, B., Schak, B., Wester, J., de Kock, C. P., and Mansvelder, H. D. (2013). Layer-specific modulation of the prefrontal cortex by nicotinic acetylcholine receptors. Cerebral Cortex, 23(1):148-161.

[Poucet et al., 2014] Poucet, B., Sargolini, F., Song, E. Y., Hangya, B., Fox, S., and Muller, R. U. (2014). Independence of landmark and self-motion-guided navigation: a different role for grid cells. Phil. Trans. R. Soc. B, 369(1635):20130370.

[Poulet and Hedwig, 2007] Poulet, J. F. and Hedwig, B. (2007). New insights into corollary discharges mediated by identified neural pathways. Trends in neurosciences, 30(1):14-21.

[Proulx et al., 2014] Proulx, C. D., Hikosaka, O., and Malinow, R. (2014). Reward processing by the lateral habenula in normal and depressive behaviors. Nature neuroscience, 17(9):1146-1152.

[Qi et al., 2014] Qi, J., Zhang, S., Wang, H.-L., Wang, H., Buendia, J. d. J. A., Hoffman, A. F., Lupica, C. R., Seal, R. P., and Morales, M. (2014). A glutamatergic reward input from the dorsal raphe to ventral tegmental area dopamine neurons. Nature communications, 5:5390.

[Quintana et al., 2012] Quintana, D. S., Guastella, A. J., Outhred, T., Hickie, I. B., and Kemp, A. H. (2012). Heart rate variability is associated with emotion recognition: direct evidence for a relationship between the autonomic nervous system and social cognition. International Journal of Psychophysiology, 86(2):168-172.

[Raichle, 2015] Raichle, M. E. (2015). The brain's default mode network. Annual review of neuroscience, 38:433-447. 
[Raij and Riekki, 2012] Raij, T. T. and Riekki, T. J. (2012). Poor supplementary motor area activation differentiates auditory verbal hallucination from imagining the hallucination. Neuroimage: Clinical, 1(1):75-80.

[Rapanelli, 2017] Rapanelli, M. (2017). The magnificent two: histamine and the H3 receptor as key modulators of striatal circuitry. Progress in Neuro-Psychopharmacology and Biological Psychiatry, 73:36-40.

[Rathelot et al., 2017] Rathelot, J.-A., Dum, R. P., and Strick, P. L. (2017). Posterior parietal cortex contains a command apparatus for hand movements. Proceedings of the National Academy of Sciences, 114(16):4255-4260.

[Redgrave and Gurney, 2006] Redgrave, P. and Gurney, K. (2006). The short-latency dopamine signal: a role in discovering novel actions? Nature reviews neuroscience, 7(12):967-975.

[Reiner et al., 2010] Reiner, A., Hart, N. M., Lei, W., and Deng, Y. (2010). Corticostriatal projection neurons-dichotomous types and dichotomous functions. Frontiers in neuroanatomy, 4.

[Rempel-Clower and Barbas, 2000] Rempel-Clower, N. L. and Barbas, H. (2000). The laminar pattern of connections between prefrontal and anterior temporal cortices in the rhesus monkey is related to cortical structure and function. Cerebral Cortex, 10(9):851865.

[Resendez et al., 2016] Resendez, S. L., Keyes, P. C., Day, J. J., Hambro, C., Austin, C. J., Maina, F. K., Eidson, L. N., Porter-Stransky, K. A., Nevárez, N., McLean, J. W., et al. (2016). Dopamine and opioid systems interact within the nucleus accumbens to maintain monogamous pair bonds. ELife, 5:e15325.

[Reyes et al., 2011] Reyes, B., Carvalho, A., Vakharia, K., and Van Bockstaele, E. (2011). Amygdalar peptidergic circuits regulating noradrenergic locus coeruleus neurons: linking limbic and arousal centers. Experimental neurology, 230(1):96-105.

[Reyes et al., 2017] Reyes, B. A., Kravets, J., Connelly, K., Unterwald, E., and Van Bockstaele, E. (2017). Localization of the delta opioid receptor and corticotropin-releasing factor in the amygdalar complex: role in anxiety. Brain Structure and Function, 222(2):1007-1026.

[Richter et al., 2017] Richter, C. G., Thompson, W. H., Bosman, C. A., and Fries, P. (2017). Top-down beta enhances bottom-up gamma. Journal of Neuroscience, pages 3771-16.

[Rivera et al., 2004] Rivera, C., Voipio, J., Thomas-Crusells, J., Li, H., Emri, Z., Sipilä, S., Payne, J. A., Minichiello, L., Saarma, M., and Kaila, K. (2004). Mechanism of activitydependent downregulation of the neuron-specific $\mathrm{K}-\mathrm{Cl}$ cotransporter KCC2. Journal of Neuroscience, 24(19):4683-4691.

[Rizzolatti and Sinigaglia, 2008] Rizzolatti, G. and Sinigaglia, C. (2008). Mirrors in the brain: How our minds share actions and emotions. Oxford University Press, USA.

[Robinson and Sohal, 2017] Robinson, S. E. and Sohal, V. S. (2017). Dopamine D2 receptors modulate pyramidal neurons in mouse medial prefrontal cortex through a stimulatory G-protein pathway. Journal of Neuroscience, 37(42):10063-10073. 
[Root et al., 2015] Root, D. H., Hoffman, A. F., Good, C. H., Zhang, S., Gigante, E., Lupica, C. R., and Morales, M. (2015). Norepinephrine activates dopamine D4 receptors in the rat lateral habenula. Journal of Neuroscience, 35(8):3460-3469.

[Roozendaal and McGaugh, 2011] Roozendaal, B. and McGaugh, J. L. (2011). Memory modulation. Behavioral neuroscience, 125(6):797.

[Rossi et al., 2008] Rossi, S., De Chiara, V., Musella, A., Kusayanagi, H., Mataluni, G., Bernardi, G., Usiello, A., and Centonze, D. (2008). Chronic psychoemotional stress impairs cannabinoid-receptor-mediated control of GABA transmission in the striatum. Journal of Neuroscience, 28(29):7284-7292.

[Rossi-Durand, 2002] Rossi-Durand, C. (2002). The influence of increased muscle spindle sensitivity on achilles tendon jerk and h-reflex in relaxed human subjects. Somatosensory \& motor research, 19(4):286-295.

[Roumis and Frank, 2015] Roumis, D. K. and Frank, L. M. (2015). Hippocampal sharpwave ripples in waking and sleeping states. Current opinion in neurobiology, 35:6-12.

[Rudy et al., 2011] Rudy, B., Fishell, G., Lee, S., and Hjerling-Leffler, J. (2011). Three groups of interneurons account for nearly $100 \%$ of neocortical GABAergic neurons. Developmental neurobiology, 71(1):45-61.

[Rutledge et al., 2015] Rutledge, R. B., Skandali, N., Dayan, P., and Dolan, R. J. (2015). Dopaminergic modulation of decision making and subjective well-being. Journal of Neuroscience, 35(27):9811-9822.

[Saalmann and Kastner, 2014] Saalmann, Y. B. and Kastner, S. (2014). Neural mechanisms of spatial attention in the visual thalamus. The Oxford Handbook of Attention, page 399.

[Sakurada et al., 1999] Sakurada, T., Katsuyama, S., Sakurada, S., Inoue, M., Tan-No, K., Kisara, K., Sakurada, C., Ueda, H., and Sasaki, J. (1999). Nociceptin-induced scratching, biting and licking in mice: involvement of spinal NK1 receptors. British journal of pharmacology, 127(7):1712-1718.

[Sakurai, 2014] Sakurai, T. (2014). The role of orexin in motivated behaviours. Nature Reviews Neuroscience, 15(11):719-731.

[Salamone and Correa, 2012] Salamone, J. D. and Correa, M. (2012). The mysterious motivational functions of mesolimbic dopamine. Neuron, 76(3):470-485.

[Santana and Artigas, 2017] Santana, N. and Artigas, F. (2017). Expression of serotonin2C receptors in pyramidal and GABAergic neurons of rat prefrontal cortex: a comparison with striatum. Cerebral Cortex, 27(6):3125-3139.

[Santello and Nevian, 2015] Santello, M. and Nevian, T. (2015). Dysfunction of cortical dendritic integration in neuropathic pain reversed by serotoninergic neuromodulation. Neuron, 86(1):233-246.

[Sarter et al., 2016] Sarter, M., Lustig, C., Berry, A. S., Gritton, H., Howe, W. M., and Parikh, V. (2016). What do phasic cholinergic signals do? Neurobiology of Learning and Memory, 130:135-141. 
[Schacter et al., 2017] Schacter, D. L., Addis, D. R., and Szpunar, K. K. (2017). Escaping the past: Contributions of the hippocampus to future thinking and imagination. In The Hippocampus from Cells to Systems, pages 439-465. Springer.

[Schindler et al., 2012] Schindler, A. G., Messinger, D. I., Smith, J. S., Shankar, H., Gustin, R. M., Schattauer, S. S., Lemos, J. C., Chavkin, N. W., Hagan, C. E., Neumaier, J. F., et al. (2012). Stress produces aversion and potentiates cocaine reward by releasing endogenous dynorphins in the ventral striatum to locally stimulate serotonin reuptake. Journal of Neuroscience, 32(49):17582-17596.

[Schlerf et al., 2012] Schlerf, J., Ivry, R. B., and Diedrichsen, J. (2012). Encoding of sensory prediction errors in the human cerebellum. Journal of Neuroscience, 32(14):49134922.

[Schoenemann et al., 2005] Schoenemann, P. T., Sheehan, M. J., and Glotzer, L. D. (2005). Prefrontal white matter volume is disproportionately larger in humans than in other primates. Nature neuroscience, 8(2):242-252.

[Schomers et al., 2015] Schomers, M. R., Kirilina, E., Weigand, A., Bajbouj, M., and Pulvermüller, F. (2015). Causal influence of articulatory motor cortex on comprehending single spoken words: Tms evidence. Cerebral cortex (new York, Ny: 1991), 25(10):3894-3902.

[Schulkin, 2017] Schulkin, J. (2017). The CRF Signal: Uncovering an Information Molecule. Oxford University Press.

[Schultz, 2016] Schultz, W. (2016). Dopamine reward prediction-error signalling: a twocomponent response. Nature Reviews Neuroscience.

[Seamans and Yang, 2004] Seamans, J. K. and Yang, C. R. (2004). The principal features and mechanisms of dopamine modulation in the prefrontal cortex. Progress in neurobiology, 74(1):1-58.

[Segaert et al., 2012] Segaert, K., Menenti, L., Weber, K., Petersson, K. M., and Hagoort, P. (2012). Shared syntax in language production and language comprehension - an fMRI study. Cerebral Cortex, 22(7):1662-1670.

[Sewards and Sewards, 2003] Sewards, T. V. and Sewards, M. A. (2003). Fear and powerdominance motivation: proposed contributions of peptide hormones present in cerebrospinal fluid and plasma. Neuroscience \& Biobehavioral Reviews, 27(3):247-267.

[Shahrokh et al., 2010] Shahrokh, D. K., Zhang, T.-Y., Diorio, J., Gratton, A., and Meaney, M. J. (2010). Oxytocin-dopamine interactions mediate variations in maternal behavior in the rat. Endocrinology, 151(5):2276-2286.

[Shan et al., 2015] Shan, Q., Christie, M. J., and Balleine, B. W. (2015). Plasticity in striatopallidal projection neurons mediates the acquisition of habitual actions. European Journal of Neuroscience, 42(4):2097-2104.

[Shang et al., 2015] Shang, C., Liu, Z., Chen, Z., Shi, Y., Wang, Q., Liu, S., Li, D., and Cao, P. (2015). A parvalbumin-positive excitatory visual pathway to trigger fear responses in mice. Science, 348(6242):1472-1477. 
[Sheets et al., 2011] Sheets, P. L., Suter, B. A., Kiritani, T., Chan, C. S., Surmeier, D. J., and Shepherd, G. M. (2011). Corticospinal-specific HCN expression in mouse motor cortex: Ih-dependent synaptic integration as a candidate microcircuit mechanism involved in motor control. Journal of neurophysiology, 106(5):2216-2231.

[Shen et al., 2008] Shen, W., Flajolet, M., Greengard, P., and Surmeier, D. J. (2008). Dichotomous dopaminergic control of striatal synaptic plasticity. Science, 321(5890):848851.

[Sherman and Guillery, 2013] Sherman, S. M. and Guillery, R. W. (2013). Functional connections of cortical areas: a new view from the thalamus. MIT Press.

[Shin et al., 2010] Shin, A. C., Pistell, P. J., Phifer, C. B., and Berthoud, H.-R. (2010). Reversible suppression of food reward behavior by chronic mu-opioid receptor antagonism in the nucleus accumbens. Neuroscience, 170(2):580-588.

[Sinton, 2008] Sinton, C. M. (2008). Neurophysiological aspects of the regulation of serotonin neurons by the orexinergic system. In Serotonin and Sleep: Molecular, Functional and Clinical Aspects, pages 287-304. Springer.

[Sippy et al., 2015] Sippy, T., Lapray, D., Crochet, S., and Petersen, C. C. (2015). Celltype-specific sensorimotor processing in striatal projection neurons during goal-directed behavior. Neuron, 88(2):298-305.

[Smith et al., 2016] Smith, A. S., Tabbaa, M., Lei, K., Eastham, P., Butler, M. J., Linton, L., Altshuler, R., Liu, Y., and Wang, Z. (2016). Local oxytocin tempers anxiety by activating GABA A receptors in the hypothalamic paraventricular nucleus. Psychoneuroendocrinology, 63:50-58.

[Smith et al., 2014] Smith, Y., Galvan, A., Ellender, T. J., Doig, N., Villalba, R. M., Huerta-Ocampo, I., Wichmann, T., and Bolam, J. P. (2014). The thalamostriatal system in normal and diseased states. Frontiers in systems neuroscience, 8.

[Sominsky and Spencer, 2014] Sominsky, L. and Spencer, S. J. (2014). Eating behavior and stress: a pathway to obesity. Frontiers in psychology, 5.

[Sommer et al., 2014] Sommer, W. H., Costa, R. M., and Hansson, A. C. (2014). Dopamine systems adaptation during acquisition and consolidation of a skill. Frontiers in integrative neuroscience, 8:87.

[Spreng et al., 2010] Spreng, R. N., Stevens, W. D., Chamberlain, J. P., Gilmore, A. W., and Schacter, D. L. (2010). Default network activity, coupled with the frontoparietal control network, supports goal-directed cognition. Neuroimage, 53(1):303-317.

[Stephenson-Jones et al., 2013] Stephenson-Jones, M., Kardamakis, A. A., Robertson, B., and Grillner, S. (2013). Independent circuits in the basal ganglia for the evaluation and selection of actions. Proceedings of the National Academy of Sciences, 110(38):E3670 E3679.

[Stoop, 2012] Stoop, R. (2012). Neuromodulation by oxytocin and vasopressin. Neuron, 76(1):142-159. 
[Suter and Shepherd, 2015] Suter, B. A. and Shepherd, G. M. (2015). Reciprocal interareal connections to corticospinal neurons in mouse M1 and S2. Journal of Neuroscience, 35(7):2959-2974.

[Taki et al., 2000] Taki, K., Kaneko, T., and Mizuno, N. (2000). A group of cortical interneurons expressing $\mu$-opioid receptor-like immunoreactivity: a double immunofluorescence study in the rat cerebral cortex. Neuroscience, 98(2):221-231.

[Tassin, 2008] Tassin, J.-P. (2008). Uncoupling between noradrenergic and serotonergic neurons as a molecular basis of stable changes in behavior induced by repeated drugs of abuse. Biochemical pharmacology, 75(1):85-97.

[Tchenio et al., 2016] Tchenio, A., Valentinova, K., and Mameli, M. (2016). Can the lateral habenula crack the serotonin code? Frontiers in synaptic neuroscience, 8.

[Tejeda et al., 2012] Tejeda, H., Shippenberg, T., and Henriksson, R. (2012). The dynorphin/ $\kappa$-opioid receptor system and its role in psychiatric disorders. Cellular and Molecular Life Sciences, 69(6):857-896.

[Terada et al., 2017] Terada, S., Sakurai, Y., Nakahara, H., and Fujisawa, S. (2017). Temporal and rate coding for discrete event sequences in the hippocampus. Neuron.

[Thomson, 2010] Thomson, A. M. (2010). Neocortical layer 6, a review. Frontiers in neuroanatomy, 4.

[Threlfell et al., 2012] Threlfell, S., Lalic, T., Platt, N. J., Jennings, K. A., Deisseroth, K., and Cragg, S. J. (2012). Striatal dopamine release is triggered by synchronized activity in cholinergic interneurons. Neuron, 75(1):58-64.

[Tian and Uchida, 2015] Tian, J. and Uchida, N. (2015). Habenula lesions reveal that multiple mechanisms underlie dopamine prediction errors. Neuron, 87(6):1304-1316.

[Timbie and Barbas, 2014] Timbie, C. and Barbas, H. (2014). Specialized pathways from the primate amygdala to posterior orbitofrontal cortex. The Journal of Neuroscience, 34(24):8106-8118.

[Toll et al., 2016] Toll, L., Bruchas, M. R., Cox, B. M., Zaveri, N. T., et al. (2016). Nociceptin/orphanin FQ receptor structure, signaling, ligands, functions, and interactions with opioid systems. Pharmacological reviews, 68(2):419-457.

[Tomasello, 2003] Tomasello, M. (2003). Constructing a language: A usage-based theory of language acquisition. Harvard University Press.

[Tovote et al., 2016] Tovote, P., Esposito, M. S., Botta, P., Chaudun, F., Fadok, J. P., Markovic, M., Wolff, S. B., Ramakrishnan, C., Fenno, L., Deisseroth, K., et al. (2016). Midbrain circuits for defensive behaviour. Nature, 534:206-212.

[Tranel et al., 1995] Tranel, D., Damasio, H., and Damasio, A. R. (1995). Double dissociation between overt and covert face recognition. Journal of Cognitive Neuroscience, 7(4):425-432.

[Tremblay et al., 2016] Tremblay, R., Lee, S., and Rudy, B. (2016). GABAergic interneurons in the neocortex: from cellular properties to circuits. Neuron, 91(2):260-292. 
[Tseng and O'donnell, 2007] Tseng, K.-Y. and O'donnell, P. (2007). Dopamine modulation of prefrontal cortical interneurons changes during adolescence. Cerebral Cortex, 17(5):1235-1240.

[Uchoa et al., 2014] Uchoa, E. T., Aguilera, G., Herman, J. P., Fiedler, J. L., Deak, T., and Sousa, M. B. C. d. (2014). Novel aspects of glucocorticoid actions. Journal of neuroendocrinology, 26(9):557-572.

[Unzai et al., 2017] Unzai, T., Kuramoto, E., Kaneko, T., and Fujiyama, F. (2017). Quantitative analyses of the projection of individual neurons from the midline thalamic nuclei to the striosome and matrix compartments of the rat striatum. Cerebral Cortex, 27(2):1164-1181.

[Uvnäs-Moberg et al., 2014] Uvnäs-Moberg, K., Handlin, L., and Petersson, M. (2014). Self-soothing behaviors with particular reference to oxytocin release induced by nonnoxious sensory stimulation. Frontiers in psychology, 5:1529-1529.

[Valentino and Van Bockstaele, 2015] Valentino, R. J. and Van Bockstaele, E. (2015). Endogenous opioids: the downside of opposing stress. Neurobiology of stress, 1:23-32.

[Van Kampen and Eckman, 2010] Van Kampen, J. M. and Eckman, C. B. (2010). Agonistinduced restoration of hippocampal neurogenesis and cognitive improvement in a model of cholinergic denervation. Neuropharmacology, 58(6):921-929.

[Van't Veer and Carlezon, 2013] Van't Veer, A. and Carlezon, W. A. (2013). Role of kappa-opioid receptors in stress and anxiety-related behavior. Psychopharmacology, 229(3):435-452.

[Veening et al., 2015] Veening, J., De Jong, T., Waldinger, M., Korte, S., and Olivier, B. (2015). The role of oxytocin in male and female reproductive behavior. European journal of pharmacology, 753:209-228.

[Veening et al., 2010] Veening, J. G., de Jong, T., and Barendregt, H. P. (2010). Oxytocinmessages via the cerebrospinal fluid: behavioral effects; a review. Physiology \& behavior, 101(2):193-210.

[Veening et al., 2012] Veening, J. G., Gerrits, P. O., and Barendregt, H. P. (2012). Volume transmission of beta-endorphin via the cerebrospinal fluid; a review. Fluids and Barriers of the CNS, 9(1):16.

[Verleger, 2008] Verleger, R. (2008). P3b: towards some decision about memory. Clinical Neurophysiology, 119(4):968-970.

[Vertes, 2015] Vertes, R. P. (2015). Major diencephalic inputs to the hippocampus: supramammillary nucleus and nucleus reuniens. circuitry and function. Progress in brain research, 219:121-144.

[Vetrivelan et al., 2016] Vetrivelan, R., Kong, D., Ferrari, L. L., Arrigoni, E., Madara, J. C., Bandaru, S. S., Lowell, B. B., Lu, J., and Saper, C. B. (2016). Melanin-concentrating hormone neurons specifically promote rapid eye movement sleep in mice. Neuroscience, 336:102-113. 
[Virk et al., 2016] Virk, M. S., Sagi, Y., Medrihan, L., Leung, J., Kaplitt, M. G., and Greengard, P. (2016). Opposing roles for serotonin in cholinergic neurons of the ventral and dorsal striatum. Proceedings of the National Academy of Sciences, 113(3):734-739.

[Viviani et al., 2011] Viviani, D., Charlet, A., van den Burg, E., Robinet, C., Hurni, N., Abatis, M., Magara, F., and Stoop, R. (2011). Oxytocin selectively gates fear responses through distinct outputs from the central amygdala. Science, 333(6038):104-107.

[Volk et al., 2012] Volk, D. W., Radchenkova, P. V., Walker, E. M., Sengupta, E. J., and Lewis, D. A. (2012). Cortical opioid markers in schizophrenia and across postnatal development. Cerebral Cortex, 22(5):1215-1223.

[Vyas, 2015] Vyas, A. (2015). Extended epigenotype in a Rattus novergicus-Toxoplasma gondii association. Communicative \& integrative biology, 8(1):e992743.

[Waldherr and Neumann, 2007] Waldherr, M. and Neumann, I. D. (2007). Centrally released oxytocin mediates mating-induced anxiolysis in male rats. Proceedings of the National Academy of Sciences, 104(42):16681-16684.

[Wall et al., 2013] Wall, N. R., De La Parra, M., Callaway, E. M., and Kreitzer, A. C. (2013). Differential innervation of direct-and indirect-pathway striatal projection neurons. Neuron, 79(2):347-360.

[Wang et al., 2007] Wang, M., Ramos, B. P., Paspalas, C. D., Shu, Y., Simen, A., Duque, A., Vijayraghavan, S., Brennan, A., Dudley, A., Nou, E., et al. (2007). a2Aadrenoceptors strengthen working memory networks by inhibiting cAMP-HCN channel signaling in prefrontal cortex. Cell, 129(2):397-410.

[Wang et al., 2017] Wang, Y., Collins, J. A., Koski, J., Nugiel, T., Metoki, A., and Olson, I. R. (2017). Dynamic neural architecture for social knowledge retrieval. Proceedings of the National Academy of Sciences, 114(16):E3305-E3314.

[Ward, 2011] Ward, L. M. (2011). The thalamic dynamic core theory of conscious experience. Consciousness and Cognition, 20(2):464-486.

[Watabe-Uchida et al., 2012] Watabe-Uchida, M., Zhu, L., Ogawa, S. K., Vamanrao, A., and Uchida, N. (2012). Whole-brain mapping of direct inputs to midbrain dopamine neurons. Neuron, 74(5):858-873.

[Wessel and Aron, 2017] Wessel, J. R. and Aron, A. R. (2017). On the globality of motor suppression: unexpected events and their influence on behavior and cognition. Neuron, 93(2):259-280.

[Wilson, 2015] Wilson, C. J. (2015). Oscillators and oscillations in the basal ganglia. The Neuroscientist, 21(5):530-539.

[Winsky-Sommerer et al., 2004] Winsky-Sommerer, R., Yamanaka, A., Diano, S., Borok, E., Roberts, A. J., Sakurai, T., Kilduff, T. S., Horvath, T. L., and de Lecea, L. (2004). Interaction between the corticotropin-releasing factor system and hypocretins (orexins): a novel circuit mediating stress response. Journal of Neuroscience, 24(50):11439-11448.

[Wittmann, 2013] Wittmann, M. (2013). The inner sense of time: how the brain creates a representation of duration. Nature Reviews Neuroscience, 14(3):217-223. 
[Woodruff et al., 2009] Woodruff, A., Xu, Q., Anderson, S. A., and Yuste, R. (2009). Depolarizing effect of neocortical chandelier neurons. Frontiers in neural circuits, 3.

[Worbe et al., 2014] Worbe, Y., Savulich, G., Voon, V., Fernandez-Egea, E., and Robbins, T. W. (2014). Serotonin depletion induces 'waiting impulsivity' on the human four-choice serial reaction time task: cross-species translational significance. Neuropsychopharmacology, 39(6):1519-1526.

[Yetnikoff et al., 2014] Yetnikoff, L., Lavezzi, H. N., Reichard, R. A., and Zahm, D. S. (2014). An update on the connections of the ventral mesencephalic dopaminergic complex. Neuroscience, 282:23-48.

[Yu et al., 2017] Yu, K., Ahrens, S., Zhang, X., Schiff, H., Ramakrishnan, C., Fenno, L., Deisseroth, K., Zhao, F., Luo, M.-H., Gong, L., et al. (2017). The central amygdala controls learning in the lateral amygdala. Nature neuroscience, 20(12):1680.

[Zhang et al., 2014] Zhang, S., Xu, M., Kamigaki, T., Do, J. P. H., Chang, W.-C., Jenvay, S., Miyamichi, K., Luo, L., and Dan, Y. (2014). Long-range and local circuits for topdown modulation of visual cortex processing. Science, 345(6197):660-665.

[Zhao et al., 2015] Zhao, H., Zhang, B.-L., Yang, S.-J., and Rusak, B. (2015). The role of lateral habenula-dorsal raphe nucleus circuits in higher brain functions and psychiatric illness. Behavioural brain research, 277:89-98.

[Zheng et al., 2007] Zheng, H., Patterson, L. M., and Berthoud, H.-R. (2007). Orexin signaling in the ventral tegmental area is required for high-fat appetite induced by opioid stimulation of the nucleus accumbens. Journal of Neuroscience, 27(41):11075-11082.

[Zhou et al., 2017] Zhou, L., Liu, M.-Z., Li, Q., Deng, J., Mu, D., and Sun, Y.-G. (2017). Organization of functional long-range circuits controlling the activity of serotonergic neurons in the dorsal raphe nucleus. Cell reports, 18(12):3018-3032.

[Zikopoulos et al., 2017] Zikopoulos, B., Höistad, M., John, Y., and Barbas, H. (2017). Posterior orbitofrontal and anterior cingulate pathways to the amygdala target inhibitory and excitatory systems with opposite functions. Journal of Neuroscience, 37(20):50515064.

[Zilles and Amunts, 2018] Zilles, K. and Amunts, K. (2018). Cytoarchitectonic and receptorarchitectonic organization in Broca's region and surrounding cortex. Current Opinion in Behavioral Sciences, 21:93-105.

[Zimmerman et al., 2017] Zimmerman, C. A., Leib, D. E., and Knight, Z. A. (2017). Neural circuits underlying thirst and fluid homeostasis. Nature Reviews Neuroscience, 18(8):459-469.

\section{Supplementary Material}

\subsection{Abbreviations and new terms}

Following is a list of abbreviations and new terms used in this text. The latter are highlighted. 
- 2-AG: 2-arachidonoyl glycerol (cannabinoid).

- abrain: the adaptive areas of the nervous system. ACC: anterior cingulate cortex. ACh: acetylcholine. ACTH: adrenocorticotropic hormone. AEA (anandamide): $\mathrm{N}$-arachidonoyl ethanolamine, (cannabinoid). AMPAR: alpha-amino-3-hydroxy-5methyl-4-isoxazolepropionic acid receptor (glut). aMTN: alpha motoneurons. ANS: autonomic nervous system. AVP: arginine vasopressin.

- BEND: beta endorphin. BF: basal forebrain. BG: basal ganglia. BLA: basolateral complex of the amygdala. BNST: bed nucleus of the stria terminalis. BU: bottom-up.

- CA1, CA2, CA3: hippocampal sub-fields. CCN: competition coordination network. CeA: central nucleus of the amygdala. CeL: lateral part of the central nucleus of the amygdala. CeM: medial part of the central nucleus of the amygdala. CER: cerebellum. CN: coordination network. Conditional: waiting for a specific event to occur.

Content (flow) networks: innate sensory network, innate response network, innate prediction network, adaptive sensory network, thalamic BU network, alert network, DM network, predecision network, prediction (goal, attention, preparation, intention) network, adaptive response network, reality network.

$\mathrm{CP}$ : calcium-permeable. CRH: corticotropin releasing hormone (or factor, $\mathrm{CRF}$ ). CS: conditioned stimulus. CSF: cerebrospinal fluid.

- DA: dopamine (D1, D2 receptors). DG: dentate gyrus (a hippocampal sub-field). DHEA: dihytoepiandrosterone. DM: decision making. DOR: delta opioid receptor. DRN: dorsal raphe nucleus. DYN: dynorphin.

- ECB: endogenous cannabinoid. ECN: execution coordination network. EEG: electroencephalography. ENK: enkephalin. ERP: event-related potential. fMRI: functional magnetic resonance imaging.

- GABA: gamma-aminobutyric acid. GC: glucocorticoid. GIRK: G protein-coupled inwardly-rectifying potassium channel. glut: glutamate, l-glutamate. gMTN: gamma motoneuron. GnRh: gonadotropin-releasing hormone. Goaling: activating nodes in prediction mode. GP: globus pallidus (GPi: internus, GPe: externus). GPRC: Gprotein coupled receptor. GR: glucocorticoid receptor.

- Hb: habenula. HT: hypothalamus.

- ibrain: the innate areas of the nervous system. ICMs: intercalated masses (amygdala). ICN: innate coordination network. IIN: inhibitory interneuron.

- JOS: join or stop (coordination).

- KISS1: kisspeptin. KOR: kappa opioid receptor.

- LC: locus coeruleus. LH: lateral hypothalamus, luteinizing hormone. LHb: lateral habenula. LSK areas: lexicon, syntax, knowledge language areas.

- mAChR: muscarinic ACh receptor. MC: microcolumn. $\mathrm{MCH}$ : melanin-concentrating hormone. MHb: medial habenula. Mobilization: activation of other non-quax neurons. MOR: mu opioid receptor. MPOA: medial preoptic area. MR: mineralocorticoid receptor. MRN: median raphe nucleus. MTN: motoneuron. 
- N modes: $N$ process stages: sensory input, innate response, learned (automated) response, acute response, alert response, extended alert, interaction, decision making, focused execution, protection, continue, consume, satisfaction, running, aggression, failure. Also mistake, internal needs. $\mathbf{N}$ process: the need process.

nAChR: nicotinic ACh receptor. NE: norepinephrine (noradrenaline). NER: norepinephrine receptor. NMDAR: N-methyl-D-aspartate glut receptor. Node: the basic cortical representation unit. NOP: nociceptin opioid. NTS: nucleus of the solitary tract.

- OFC: orbitofrontal cortex. OOA: orienting of attention. OT: oxytocin. OX: orexin (hypocretin).

- PAG. periaqueductal gray. PFC: prefrontal cortex (mPFC: medial, vmPFC: ventromedial). POMC: proopiomelanocortin. PV: parvalbumin. PVH: paraventricular hypothalamus.

- Q process: the quax process. Quax: neurons active in a sensor-response mapping.

- R17: the theory presented here. $\mathbf{R}$ mode: $\mathrm{R}$ process mode. $\mathbf{R}$ process: the main process. REMS: rapid eye movement sleep. Rgen: $\mathrm{R}$ process agent. RMTg: rostromedial tegmental nucleus (VTA). RSC: retrosplenial cortex.

- SC: superior colliculus. Scaffold: the inhibitory CN neurons supporting a quax. SER: serotonin. Skeleton: the excitatory neurons of a quax. SN: subtantia nigra (SNr: reticulata, SNc: compacta). SOM: somatostatin. SR: sensor-response. STR: the striatum (dSTR: dorsal, vSTR: ventral). SP: substance P. SWRs: short wave ripples.

- TD: top-down. Transition: the joining and leaving of neurons from a quax.

- UQS: underlying quax space. US: unconditioned stimulus.

- VIP: vasoactive intestinal polypeptide. VP: ventral palllidum. VTA: ventral tegmental area.

\subsection{Method}

To gather as many relevant facts as possible, I systematically examined papers and books. The papers include almost all of the papers published in the last ten years in the journals listed below, many papers that they cite, and all of the papers citing the most relevant papers. This resulted in about 15,000 examined papers. The only search tool I used was Google Scholar, for citations and focused searches.

Following are the journals that were throughly examined, in no particular order. In addition to these, many journals were examined less systematically.

Neuron, The Journal of Neuroscience, Nature Neuroscience, Nature Reviews Neuroscience, PNAS, Cell, Science, Current Opinion in Neurobiology, Trends in Neurosciences, Trends in Cognitive Sciences, Neuroscience \& Biobehavioral Reviews, Behavioural Brain Research, The Journal of Cognitive Neuroscience, Brain, Cerebral Cortex, Neuroimage, Neuroimage: Clinical, Human Brain Mapping, Brain Research, Cortex, Annual Review of Neuroscience, Annual Review of Psychology, Progress in Brain Research, Progress in Neurobiology, Journal of Learning and Memory, Frontiers in Neuroscience, Frontiers in Psychology, Frontiers in Neurology, Frontiers in Psychiatry, Current Biology, European 
Journal of Neuroscience, Neuropsychopharmacology, Moleclar Psychiatry, Biological Psychiatry, The Journal of Physiology, Philosophical Transactions of the Royal Society B, Cold Spring Harbor Perspectives in Biology, CSH Learning \& Memory, The Neuroscientist, Neuropsychologia, Brain Structure and Function, Journal of Experimental Psychology (General, Learning Memory and Cognition, Applied, Human Perception and Performance), Hippocampus, Brain and Cognition, Neuroscience, Experimental Brain Research, eLife, Journal of Comparative Neurology, Psychopharmacology, Physiological Reviews, Journal of Psychophysiology, Neuropsychology, Topics in Cognitive Science, Nature, PLoS ONE.

\subsection{Generative linguistics, recursion, etc.}

At present, most undergraduate teaching in linguistics and a substantial part of linguistics research involves the so-called 'generative linguistics' (GL) doctrine. Most if not all of the central tenets of this doctrine are strongly disputed by leading linguists and cognitive scientists (e.g., Basic Linguistic Theory [Dixon, 2005], Cognitive Grammar [Langacker, 2008], Construction approaches [Goldberg, 2006], the Parallel Architecture [Jackendoff, 2002], a view from child language acquisition [Tomasello, 2003], a view from the uniqueness of language [Pinker and Jackendoff, 2005]). Some of the tenets have been abandoned by GL supporters. I devote a few paragraphs to GL here because its tenets still appear as truths in linguistics textbooks and because some of them are widely known to the general public.

R17 implies that virtually all of the basic GL tenets stated during the last decades are completely wrong. There is no 'innate' capacity specific for syntax, because syntax uses the general Q process architecture of the brain. There is no 'poverty of the stimulus', since generalizations over simpler representations acquired through comprehensible input can be done just as they are done in all other modalities. There are no specific syntactic universals, because there are many types of auditory sequences that can be automated and that satisfy the motivational needs. The notion of 'transformations' has no central role in language and probably stems from an English-centric analysis. The distinction between 'performance' and 'competence' is misleading, because every utterance that a person makes is a direct result of their brain representations at the time of speaking, and yields the update of these representations. Basing a theory on categorial measures of 'correctness' ignores the facts that acceptability is a continuous spectrum and that the degree of perceived correctness depends on literacy, and contradicts mechanisms of language change. The large expressive power of language is due to the huge number of possible word sequences, which is in turn due to the large number of words and not to any inherent qualitative uniqueness of language. Finally, the brain does not implement recursion, as shown in some detail below. There is basically no experimental evidence that supports these GL tenets, and much evidence that contradicts them (see references above). Other GL tenets include the obvious observation (already made by ancient Greek grammarians) that language is hierarchical (words, phrases, clauses), and the trivial observation that its significant event is the appearance of a new word ('the minimalist program').

One of the tenets of generative linguistics is that one of the things (or the thing) unique to language is recursion. Recursion means that there is an action operating on some input, and during its execution the same action is activated on some other input (usually derived from the original input). The execution of actions can obviously involve the execution of other actions; the point in recursion is that it is the same action that is being activated (with different inputs, otherwise we get into an infinite loop).

The claim to fame of recursion in language is that it supports an unlimited number of embedded clauses, for example in sentences such as ' $\mathrm{X}$ said that $\mathrm{Y}$ said that $\mathrm{Z}$ said that...'. 
However, people very quickly (after two or three recursion levels) lose the meaning of such sentences; understanding deep recursive sentences involves a conscious mental effort and usually cannot be done without slow rehearsal. After just a few recursion levels, the only way to understand such sentences is to write them down, showing that the brain finds it particularly hard to implement recursion. This is a simple result of the Q process, because recursion requires the same node to maintain an arbitrary number of different open goals, which is exactly the situation that imposes strict limitations on the number of items in working memory (see WM above). Indeed, converging evidence supports the proposal that automated language use (syntax, semantics) relies on a specialized WM-like capacity [Caplan and Waters, 1999].

There is solid experimental support for the argument above from the 'missing VP' paradigm. Consider the following two sentences. Which one seems more natural immediately during listening (i.e., before re-thinking about their meanings)?

1. "The patient who the nurse who the clinic hired met jack".

2. "The patient who the nurse who the clinic hired admitted met jack".

It has been repeatedly shown (e.g., in English [Gibson and Thomas, 1999] and French [Gimenes et al., 2009]) that (1) sounds more natural than (2), even though it is syntactically incorrect. Syntactic predictions for the 2 nd verb in (2) ('admitted') are created by the syntax node triggered by 'the nurse'. The phrase 'who the nurse' is followed by 'who the clinic', which, since English does not use two consecutive 'who' patterns, is represented using the same syntactic action nodes. Hence, this is a case where recursion should be used if it is used at all. However, the appearance of 'admitted' sounds strange, and a plausible account for this is that the predictions (goals) for it have been overwritten after 'who the clinic' was complemented by 'hired'. Sentence (1) sounds natural because all words are from the same general semantic domain (so create anticipations that conform to the input words), while no syntax nodes are left with unanswered goals.

Note that the above does not imply that languages cannot use such patterns. It only implies that they cannot use recursion (i.e., the same node) to represent them. Indeed, embedding a clause within another is very common, and this is very simple to implement by each level being suppoted by different syntax nodes. A sequence of three consecutive main (non-auxiliary) verbs is not used in English, but it exists in Dutch and German, and the experiment above indeed yields less convincing results in these languages [Frank et al., 2015]. 